\title{
Primer consenso nacional de edema macular diabético
}

\section{First national consensus on diabetic macular edema}

\author{
Juan Manuel Jiménez-Sierra ${ }^{1 *}$, René Cano-Hidalgo², Vanessa Flores-Peredo ${ }^{3}$, Jans Fromow-Guerra1, \\ Gerardo García-Aguirre ${ }^{1}$, Renata García-Franco ${ }^{4}$, Jesús González-Cortés ${ }^{5}$, Sergio Hernández-Da Mota , \\ Fabiola Hernández-Núñez ${ }^{7}$ Ángela Hernández-Vázquez ${ }^{8}$, Gabriela Lopezcarasa-Hernández ${ }^{9}$, \\ David Lozano-Rechy², Ximena Mira-Lorenzo ${ }^{10}$, Carla Pérez-Montaño ${ }^{8}$, Abel Ramírez-Estudillo ${ }^{8}$, \\ Patricio Rodríguez-Valdéz ${ }^{11}$, Enrique Roig-Melo ${ }^{12}$ y Ermilo Sánchez-Buenfil ${ }^{13}$
}

${ }^{1}$ Asociación para Evitar la Ceguera en México (APEC), Ciudad de México; ${ }^{2}$ nstituto de Oftalmología FAP Conde de Valenciana, Ciudad de México; ${ }^{3}$ Hospital Regional Adolfo López Mateos, Instituto de Seguridad y Servicios Sociales de los Trabajadores del Estado, Ciudad de México; ${ }^{4}$ nstituto de la Retina del Bajío INDEREB, Querétaro, Querétaro; ${ }^{5}$ Hospital Universitario José Eleuterio González, Universidad Autónoma de Nuevo León,

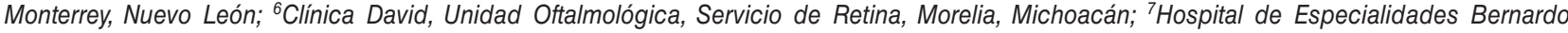

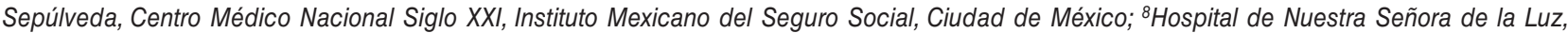
Ciudad de México; ${ }^{9}$ Hospital Ángeles Lomas, Instituto de Ciencias Oftalmológicas, Hacienda de las Palmas, Estado de México; ${ }^{10} \mathrm{Centro}$ Oftalmológico Arcos, Querétaro, Querétaro; ${ }^{11}$ Instituto de Oftalmología y Ciencias Visuales, Tecnológico de Monterrey, Nuevo León; ${ }^{12}$ Instituto de Investigación y Ciencias Visuales, Universidad de Guadalajara, Guadalajara, Jalisco; ${ }^{13}$ Centro de Oftalmología Especializada Retimediq, Mérida, Yucatán. México

\section{Resumen}

El edema macular diabético (EMD) representa la causa principal de pérdida de la visión en pacientes con diabetes mellitus. Se trata de una enfermedad compleja que demanda a los oftalmólogos y retinólogos una amplia experiencia para lograr una oportuna detección, un certero diagnóstico y un adecuado tratamiento. En la actualidad se cuenta con diversas y novedosas alternativas de tratamiento que deben ser evaluadas a la par que las características y las necesidades de cada paciente para brindarle los mayores beneficios. En este documento se describen las características y la evidencia científica que respaldan algunos de estos tratamientos, así como la propuesta del Grupo de Consenso para el tratamiento más apropiado de los pacientes con EMD de acuerdo con sus características y perfiles.

Palabras clave: Edema macular diabético. Tratamiento. Anti-VEGF. Esteroide. Agudeza visual. Calidad de vida.

\begin{abstract}
Diabetic macular edema (DME) represents the leading cause of vision loss in patients with diabetes mellitus. It is a complex disease that demands extensive experience from ophthalmologists and retinologists to achieve a timely detection, an accurate diagnosis and adequate treatment. At present, there are various and innovative treatment alternatives that must be evaluated along with the characteristics and needs of each patient to provide the greatest benefits. This document describes the characteristics and scientific evidence that support some of these treatments, as well as the Consensus Group's proposal for the most appropriate treatment of patients with DME according to their characteristics and profiles.
\end{abstract}

Key words: Diabetic macular edema. Treatment. Anti-VEGF. Steroid. Visual acuity. Quality of life.

\section{Correspondencia:}

*Juan Manuel Jiménez-Sierra

Vicente García Torres 46, Barrio de San Lucas,

Coyoacán

Fecha de recepción: 29-06-2021

Fecha de aceptación: 26-08-2021

C.P. 04030, Ciudad de México

E-mail: dr.jimenezsierra@gmail.com
Disponible en internet: 10-11-2021 Rev Mex Oftalmol. 2021;95(Supl 1):1-118 www.rmo.com.mx (http://creativecommons.org/licenses/by-nc-nd/4.0/). 


\section{Prólogo}

El edema macular diabético es uno de los problemas a los que con más frecuencia se enfrenta un oftalmólogo, pero no por eso es una enfermedad fácil de diagnosticar o tratar. El hecho de que existan tantas variables que influyen en la manera en que este padecimiento se manifiesta, y que se disponga de diversas modalidades de tratamiento, hacen que el manejo de los pacientes con este diagnóstico sea un reto significativo para el clínico, que requiere, la mayoría de las veces, un régimen individualizado y hecho a la medida de las necesidades de cada paciente.

El Dr. Juan Manuel Jiménez Sierra se dio a la tarea de coordinar la participación de varios expertos en el tema, todos miembros de la Asociación Mexicana de Retina, quienes en conjunto han elaborado el Primer Consenso Nacional de Edema Macular Diabético. Este documento aborda distintos aspectos del diagnóstico y del manejo de esta patología, permitiendo al lector conocer su epidemiología, fisiopatología y clasificación, la utilidad de los distintos métodos diagnósticos disponibles, la selección de los pacientes que pueden beneficiarse del tratamiento, las distintas opciones de manejo (incluyendo láser, anti-VEGF, esteroides y cirugía) y los criterios para decidir cambiar el tipo de tratamiento.

El resultado de esta colaboración es un consenso que pretende servir al oftalmólogo como una guía clara y concisa que resume el conocimiento que hasta ahora tenemos de esta enfermedad, con la finalidad de poder acceder a una fácil revisión actualizada del tema, que pueda facilitar la toma de decisiones y, por lo tanto, impactar positivamente en la visión y la calidad de vida de los pacientes. Estoy seguro de que el lector lo encontrará de suma utilidad.

Dr. Gerardo García Aguirre

Presidente de la Asociación Mexicana de Retina, 2020

\section{Objetivos}

Actualizar, documentar y emitir recomendaciones sobre el diagnóstico clínico, los estudios diagnósticos y las modalidades de tratamiento actual para el edema macular diabético (EMD), según la evidencia clínica publicada y la experiencia clínica de un grupo de retinólogos mexicanos expertos en el tema.

\section{Método}

A partir de una reunión inicial con un grupo de médicos especialistas expertos en EMD, llevada a cabo en el mes de octubre de 2019, en la ciudad de Puebla, México, se reconoció la importancia de contar con un documento que presentara los diferentes aspectos clínicos, de diagnóstico y de tratamiento del EMD. Así, se planteó la necesidad de elaborar un Consenso Nacional que presentara la evidencia clínica publicada y la experiencia clínica de un grupo de retinólogos expertos en el tema.

Como un primer paso se conformó el grupo de expertos participantes. Se contó con la participación de 18 especialistas en EMD provenientes de diferentes instituciones del país. Este grupo de expertos se encargó del desarrollo de 22 temas relacionados con el EMD. Una vez planteados los temas, estos fueron montados en una plataforma digital (Within-3) para poder ser revisados, comentados y discutidos por todos los miembros del grupo de consenso. En este documento se presentan dichos temas y los protocolos de manejo del EMD validados por el Grupo de Consenso.

\section{Agradecimientos}

El Grupo de Consenso reconoce y agradece a Allergan/AbbVie México por el compromiso y el apoyo irrestricto otorgado para la realización de este trabajo.

\section{Conflicto de intereses}

Ninguno de los autores de este Grupo de Consenso tiene conflictos de intereses en relación con los tratamientos o fármacos mencionados en esta publicación.

\section{Responsabilidades éticas}

Protección de personas y animales. Los autores declaran que para esta investigación no se han realizado experimentos en seres humanos ni en animales.

Confidencialidad de los datos. Los autores declaran que han seguido los protocolos de su centro de trabajo sobre la publicación de datos de pacientes.

Derecho a la privacidad y consentimiento informado. Los autores han obtenido el consentimiento informado de los pacientes y/o sujetos referidos en el artículo. Este documento obra en poder del autor de correspondencia. 


\title{
Epidemiología del edema macular diabético en México
}

\author{
Jans Fromow-Guerra* \\ Asociación para Evitar la Ceguera en México (APEC), Ciudad de México, México
}

La diabetes mellitus o tipo 2 (DM2) es una enfermedad con distribución universal, con una morbilidad y una mortalidad significativas, que afecta tanto a los países desarrollados como a aquellos en vías de desarrollo. En el año 2010 se calculaba que había 285 millones de personas viviendo con diabetes. Para el año 2030, esta cifra incrementará a 366 millones de personas, y para el año 2040, de los 600 millones de personas que se calcula que padecerán diabetes, unos 400-500 millones vivirán en países en vías de desarroIlo. Así, el impacto de la diabetes supone un reto para los sistemas de salud de dichos países ${ }^{1,2}$.

Las complicaciones microvasculares de la DM2, incluyendo la evolución del daño retiniano conocido como retinopatía diabética (RD), han sido reconocidas y estudiadas por muchos años. En el año 2010, la RD era causa de 3.7 millones de casos de discapacidad visual y de más de 833,000 casos de ceguera irreversible en todo el mundo. A pesar de los avances tecnológicos para el diagnóstico y el tratamiento de esta complicación, la RD, que incluye el edema macular diabético $(E M D)$ y la retinopatía diabética proliferativa (RDP), continúa siendo la principal causa de pérdida visual moderada y grave irreversible en los países desarrollados ${ }^{3}$.

En el año 2016, la DM2 fue declarada emergencia epidemiológica nacional en México. En la Encuesta Nacional de Salud en México (ENSANUT) de 2016, la prevalencia total calculada de la diabetes en adultos mexicanos fue del $13.7 \%$ (12.0-15.5). Las tasas de mortalidad atribuibles a esta enfermedad se encuentran entre las más altas del mundo ${ }^{4,5}$.

La ENSANUT de 2018 estableció que el porcentaje de población de 20 años y más con diagnóstico previo de diabetes era del $10.3 \%$, frente al $9.2 \%$ en 2012. Este porcentaje fue mayor en algunos Estados de la República, como Campeche, Tamaulipas, Hidalgo, Ciudad de México y Nuevo León, donde llegó a ser del $12 \%$ al $14 \%$.

La DM2 de larga evolución y la hipertensión se han encontrado en forma consistente relacionadas con el desarrollo y la evolución de RD. En la mayoría de los pacientes con DM2, la edad de presentación de la RD es a los 10-15 años de realizado el diagnóstico ${ }^{3,5}$.

Actualmente, la RD se encuentra en el tercer lugar en cuanto a costos directos generados por complicaciones de la DM2 en México. En este contexto, se espera que la epidemia de complicaciones por diabetes empeore, incluyendo la RD. En la ENSANUT de 2018 se reporta que tanto en la encuesta de 2012 como en la de 2018 la complicación mayormente referida en los pacientes con diabetes en México es la visión disminuida 6 .

En cuanto al EMD, existen variaciones significativas respecto a la incidencia y la prevalencia de esta complicación de la DM2 alrededor del mundo. Esta variabilidad depende de factores como el tipo de diabetes, la modalidad de tratamiento y la duración media de la diabetes. En el estudio epidemiológico de retinopatía diabética de Wisconsin XXIII, la tasa de desarrollo de EMD a 25 años 
Tabla 1. Cálculos del número total de enfermos de retinopatía diabética en grados tratables con fotocoagulación y edema macular diabético

\begin{tabular}{|c|c|c|c|c|c|c|c|}
\hline \multirow[t]{2}{*}{ Edad (años) } & \multirow{2}{*}{$\begin{array}{c}\text { Porcentaje de } \\
\text { población }\end{array}$} & \multirow{2}{*}{$\begin{array}{l}\text { Población } \\
\text { total }\end{array}$} & \multirow{2}{*}{$\begin{array}{c}\text { Prevalencia } \\
\text { diabetes } \\
\text { ENSANUT } 2018\end{array}$} & \multirow{2}{*}{$\begin{array}{c}\text { Total población } \\
\text { DM2 }\end{array}$} & \multicolumn{3}{|c|}{ Número pacientes esperados en México } \\
\hline & & & & & RDNP/RDP & EMD & EMCS \\
\hline $\begin{array}{l}0-10 \\
10-19 \\
20-29 \\
30-39 \\
40-49 \\
50-59 \\
60-69 \\
70 \text { o más }\end{array}$ & $\begin{array}{c}16.1 \% \\
18.2 \% \\
15.6 \% \\
13.8 \% \\
13.3 \% \\
10.6 \% \\
6.8 \% \\
5.5 \%\end{array}$ & $\begin{array}{c}20,125,000 \\
22,750,000 \\
19,500,000 \\
17,250,000 \\
16,625,000 \\
13,250,000 \\
8,500,000 \\
6,875,000 \\
125,000,000\end{array}$ & $\begin{array}{c}3.3 \% \\
3.2 \% \\
13.8 \% \\
26.9 \% \\
36.5 \% \\
25.5 \%\end{array}$ & $\begin{array}{c}643,500 \\
552,000 \\
2,294,250 \\
3,564,250 \\
3,102,500 \\
1,753,125 \\
11,909,625\end{array}$ & $\begin{array}{c}- \\
6,458 \\
42,504 \\
62,928 \\
62,928 \\
67,896 \\
242,714\end{array}$ & $\begin{array}{c}- \\
11,040 \\
48,024 \\
65,136 \\
59,616 \\
54,648 \\
238,464\end{array}$ & $\begin{array}{c}- \\
6,955 \\
30,360 \\
34,224 \\
38,088 \\
35,328 \\
144,955\end{array}$ \\
\hline
\end{tabular}

DM2: diabetes mellitus tipo 2; EMD: edema macular diabético; EMCS: edema macular clinicamente significativo; RDNP: retinopatía diabética no proliferativa; RDP: retinopatía diabética proliferativa.

fue del $29 \%$ y la de edema macular clínicamente significativo fue del $17 \%$. La prevalencia de EMD aumenta con la gravedad de la RD: afecta al $3 \%$ de los ojos con retinopatía diabética no proliferativa (RDNP) leve, al $38 \%$ de los ojos con RDNP moderada a grave, y al $71 \%$ de los ojos con RDP. Algunos factores, como la RDNP leve, la edad, el sexo masculino y los valores altos o los incrementos de hemoglobina glucosilada, se han relacionado con la tasa de progresión acumulativa de la RD hacia estadios más graves, como la RDP y el EMD, así como a glaucoma neovascular ${ }^{7,8}$.

Los reportes de estudios poblacionales sobre cómo se manifiestan la RD y el EMD en mexicanos con diabetes los encontramos principalmente en el estudio Los Angeles Latino Eye Study (LALES). Sin embargo, este estudio muestra la prevalencia en mexicanos que viven en Los Ángeles, con las modificaciones dietéticas y epidemiológicas que esto implica. Además, solo se estudiaron pacientes mayores de 40 años. A pesar de esto, es un cálculo valioso que muestra que la prevalencia global de RD fue del $46.9 \%$, la de RDNP grave fue del $4.4 \%$ y la de RDP fue del $6.1 \%$. El EMD se observó en el $10.4 \%$ y el llamado EMD clínicamente significativo fue del $6.2 \%$ de todos los sujetos diabéticos ${ }^{9}$.

En un ejercicio atendiendo a los reportes poblacionales del Instituto Nacional de Estadística y Geografía en 2019 para los estimados de 2018 que calculan un total de población de 125 millones de mexicanos, aplicando los estimados de la ENSANUT 2018, así como las prevalencias reportadas por LALES, y ajustando correctamente por la pirámide poblacional, nos da un total de 11,909,000 pacientes con diabetes, de los cuales 1,107,000 tendrían algún grado de RD, 242,000 tendrían $\mathrm{RD}$ que requiere tratamiento con láser y 238,500 presentarían EMD (Tabla 1) ${ }^{10}$.

Estos cálculos son incluso conservadores en comparación con las prevalencias reportadas en un estudio pequeño realizado en el estado de Chiapas, que reveló que el $38.9 \%$ de los adultos mayores de 50 años con diagnóstico de DM2 tenían RD y el 21\% tenían RDP ${ }^{11}$.

\section{Bibliografía}

1. Wong T, Sun J, Kawasaki R, Ruamviboonsuk P, Gupta N, Lansingh V, et al. Guidelines on diabetic eye care: the International Council of Ophthalmology Recommendations for Screening, Follow-up, Referral and Treatment Based on Resource Settings. Ophthalmology. 2018;125: 1608-22.

2. Ting D, Cheung G, Wong T. Diabetic retinopathy: global prevalence, major risk factors, screening practices and public health challenges: a review. Clin Exp Ophthalmol. 2016:44:260-77.

3. Jampol L, Glassman A, Sun J. Evaluation and care of patients with diabetic retinopathy. N Engl J Med. 2020;382:1629-37.

4. Basto A, Barrientos T, Rojas R, Aguilar C, López N, De la Cruz V et al. Prevalence of diabetes and poor glycemic control in Mexico: results from ENSANUT 2016. Salud Publica Mex. 2020;62:50-9.

5. Mendoza K, Quezada A, Pedroza A, Hernández C, Fromow J, Barquera S. A diabetic retinopathy screening tool for low-income adults in Mexico. Prev Chronic Dis. 2016;14:E95.

6. Secretaría de Salud. Encuesta Nacional de Salud y Nutrición 2018. Presentación de resultados. (Consultado el 12 agosto 2020.) Disponible en: https://ensanut.insp.mx/encuestas/ensanut2018/doctos/informes/ensanut_2018_presentacion_resultados.pdf

7. Bandello F, Battaglia M, Lanzetta $P$, Loewenstein A, Massin P, Menchini F, et al. Diabetic macular edema. Dev Ophthalmol. 2017;58:102-38.

8. Klein R, Knudtson M, Lee K, Gangnon R, Klein B. The Wisconsin Epidemiologic Study of Diabetic Retinopathy XXIII: the twenty-five-year incidence of macular edema in persons with type 1 diabetes. Ophthalmology. 2009;116:497-503.

9. Varma R, Torres M, Pena F, Klein R, Azen S. Prevalence of diabetic retinopathy in adult Latinos: the Los Angeles Latino Eye Study. Ophthalmology. 2004;7:1298-306.

10. Hernández M, López R, Velarde S. La situación demográfica en México. Panorama desde las proyecciones de población 2013. Disponible en: http://www.conapo.gob.mx/work/models/CONAPO/Resource/1720/1/images/1_La_Situacion_Demografica_En_Mexico.pdf

11. Polack S, Yorston D, López A, Lepe S, Martins R, Alvez L, et al. Rapid assessment of avoidable blindness and diabetic retinopathy in Chiapas, Mexico. Ophthalmology. 2012;119:1033-40. 


\title{
Fisiopatología del edema macular diabético: enfermedad vascular mediada por el factor de crecimiento vascular endotelial
}

\author{
Abel Ramírez-Estudillo* \\ Hospital de Nuestra Señora de la Luz, Ciudad de México, México
}

El edema macular diabético (EMD) se reconoce como la causa principal de pérdida visual en pacientes con retinopatía diabética. Su patogénesis es multifactorial y para su presentación intervienen factores locales y sistémicos.

La alteración histológica encontrada en el EMD es una acumulación de líquido en la capa de Henle y la capa nuclear interna de la retina. Este líquido proviene del compartimento y flujo intravascular, que como en el resto de los tejidos está modulado por el equilibrio entre la presión hidrostática y la presión osmótica. Sin embargo, en la retina existe además otra estructura, la barrera hematorretiniana (BHR), que se encarga de regular el paso de algunos componentes plasmáticos libremente, y solo en condiciones patológicas puede alterar su permeabilidad, representando así el principal factor para la acumulación de líquido extracelular en la retina.

Entre los mecanismos por los cuales se lleva a cabo la rotura de la BHR se conocen los siguientes: alteración en las uniones celulares, pérdida de pericitos y células endoteliales, leucostasis (Figura 1), incremento en la permeabilidad de la superficie de membrana de la células endoteliales y del epitelio pigmentado de la retina, incremento en la activación de los receptores para productos finales de la glucosilación, disminución en la regulación del factor neurotrófico derivado de las células gliales, dilatación de los vasos retinianos y tracción vitreorretiniana.
Hasta el momento se considera que el incremento en los productos finales de glucosilación es un elemento esencial en el desarrollo del EMD, pues facilita la expresión de endotelinas, interleucina-1 (IL-1) y factor de necrosis tumoral, el aumento de colágena tipo IV en la membrana basal de la pared vascular y el daño en la fibronectina y la laminina, que son sin duda factores fundamentales en el deterioro vascular en el paciente diabético'.

Otros factores, como la hipoxia, alteran el flujo sanguíneo y facilitan el desarrollo de procesos inflamatorios que condicionan un incremento de la producción del factor de crecimiento vascular endotelial (VEGF, vascular endothelial growth factor), un aumento de la adhesión leucocitaria, una disminución del factor derivado del epitelio pigmentado y una elevación de la proteína cinasa $C(\mathrm{PKC})$, que son algunas condiciones que facilitan la progresión del EMD. La mayor susceptibilidad de la mácula a la formación de edema podría deberse al hecho de que esta zona de la retina posee muy pocos astrocitos, que son células que sintetizan factores que aumentarían la síntesis de proteínas de las uniones estrechas de la BHR y por tanto disminuirían su permeabilidad.

En un estado fisiológicamente saludable se produce agua como subproducto metabólico dentro del tejido retiniano neuronal, esto como producto final de la extracción de electrones y la generación de trifosfato de adenosina (ATP). La presión intraocular normal del 


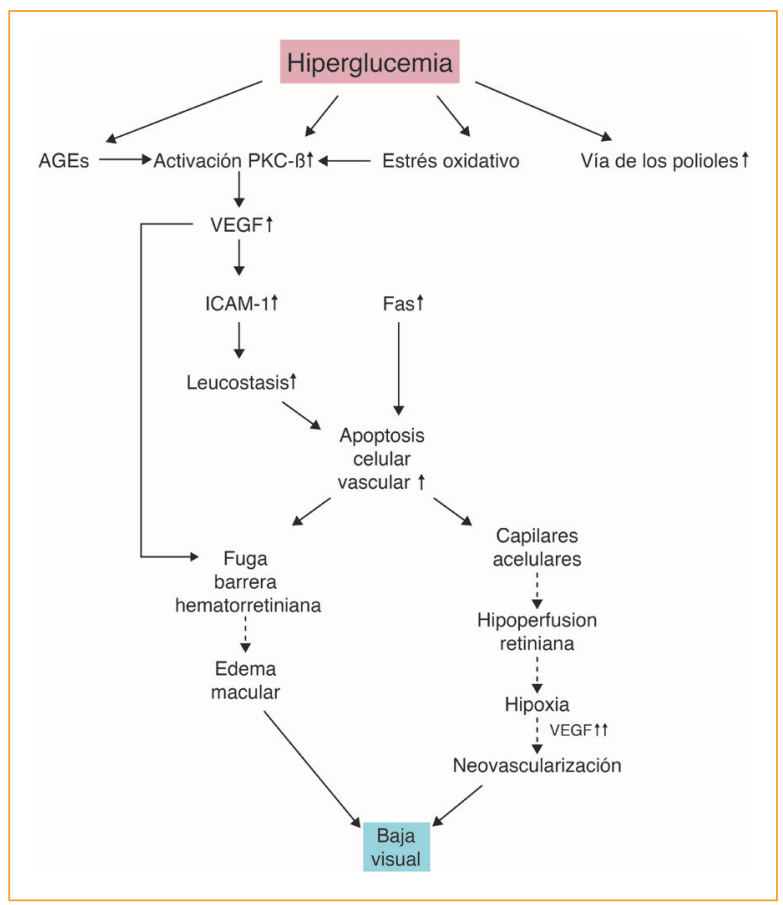

Figura 1. Diagrama del papel de la leucostasis en el desarrollo de retinopatía diabética en modelos animales. Las rutas representadas por flechas punteadas se basan en conjeturas, mientras que las flechas sólidas representan rutas demostradas. (Adaptada de www. jleukbio.org to IP.230128.122.132).

ojo envía continuamente líquido hacia la retina. La fuerza generada por este líquido que ingresa en la retina es uno de los mecanismos que la mantienen unida al epitelio pigmentado retiniano (EPR). En condiciones normales, la retina interna se mantiene deshidratada mediante las células gliales, como las de Müller, mientras que el espacio subretiniano y la retina externa se mantienen secos mediante un mecanismo de bombeo del EPR. Estas células cuentan con unos canales especiales, denominados acuaporinas, que mejoran la permeabilidad de las membranas y permiten el rápido intercambio de fluidos.

En los pacientes con diabetes mellitus tipo $1 \mathrm{y}$ tipo 2, el control glucémico deficiente conduce a hiperglucemia, que a su vez impulsa la regulación aberrante de al menos cinco vías bioquímicas clave: la ruta del poliol, la ruta de la PKC, la formación avanzada de productos finales de la glicación (AGE), la ruta de la hexosamina y la regulación positiva de la poli-(ADP-ribosa)-polimerasa. La desregulación de estas vías exacerba el estrés oxidativo, por ejemplo con una mayor producción de especies reactivas de oxígeno, lo que a su vez conduce a disfunción mitocondrial, inflamación e hipoxia. Como resultado, existe una regulación al alza del VEGF, que se ha implicado como un factor clave causante de la neovascularización retiniana y la hiperpermeabilidad vascular en la retinopatía diabética proliferativa y el EMD (Figuras 2 y 3$)^{2}$.

EI VEGF desempeña un papel crítico en el desarrollo del EMD. Está implicado en la neovascularización y en el aumento de la permeabilidad vascular. La cantidad de VEGF en las cámaras anterior y vítrea se correlaciona con la gravedad del EMD. La introducción de VEGF en un ojo de primate normal induce cambios patológicos de formación de microaneurismas y aumenta la permeabilidad vascular, que son las características que se observan en la retinopatía diabética. Estas observaciones hicieron del VEGF un objetivo discernible en el tratamiento del EMD.

Los primeros cambios vasculares en la retina que se observan en la histopatología son el engrosamiento de la membrana basal, una lesión endotelial que conduce a la interrupción de las uniones celulares y la muerte de los pericitos. Las consecuencias de la pérdida de pericitos son la desregulación del tono vascular y el crecimiento y la proliferación de las células endoteliales debido al déficit de factor de crecimiento transformante beta producido por los pericitos. Estos cambios apuntalan el desarrollo de microaneurismas y hemorragias intrarretinianas puntiformes, dos de las manifestaciones clínicas más tempranas y distintivas de la retinopatía diabética. La membrana basal engrosada es disfuncional y permite el paso del contenido intravascular (proteínas, lípidos, mediadores de la inflamación y otros constituyentes plasmáticos) al espacio intersticial. Por otro lado, el VEGF-A y las citocinas proinflamatorias (IL-1 $1 \beta$, factor de necrosis tumoral alfa, IL-6, IL-8, proteína quimiotáctica de monocitos 1 , etc.), secretadas por las células gliales (células de Müller y microglía), el EPR y los macrófagos, también tienen papeles cruciales en el deterioro microvascular precoz y la disfunción tisular. La secreción mantenida de mediadores inflamatorios puede conducir a un estado inflamatorio crónico precoz y persistente en la retina, que da como resultado una regulación positiva de los factores de adhesión, como la molécula de adhesión intercelular 1 (ICAM-1), en la superficie de la célula endotelial, la activación de los leucocitos y la adhesión al endotelio (Figura 4) ${ }^{3}$.

\section{Ley de Starling}

En general, se acepta que la causa inmediata del edema macular y de la acumulación de líquido en la retina es una rotura de la BHR. Por lo tanto, los principales 


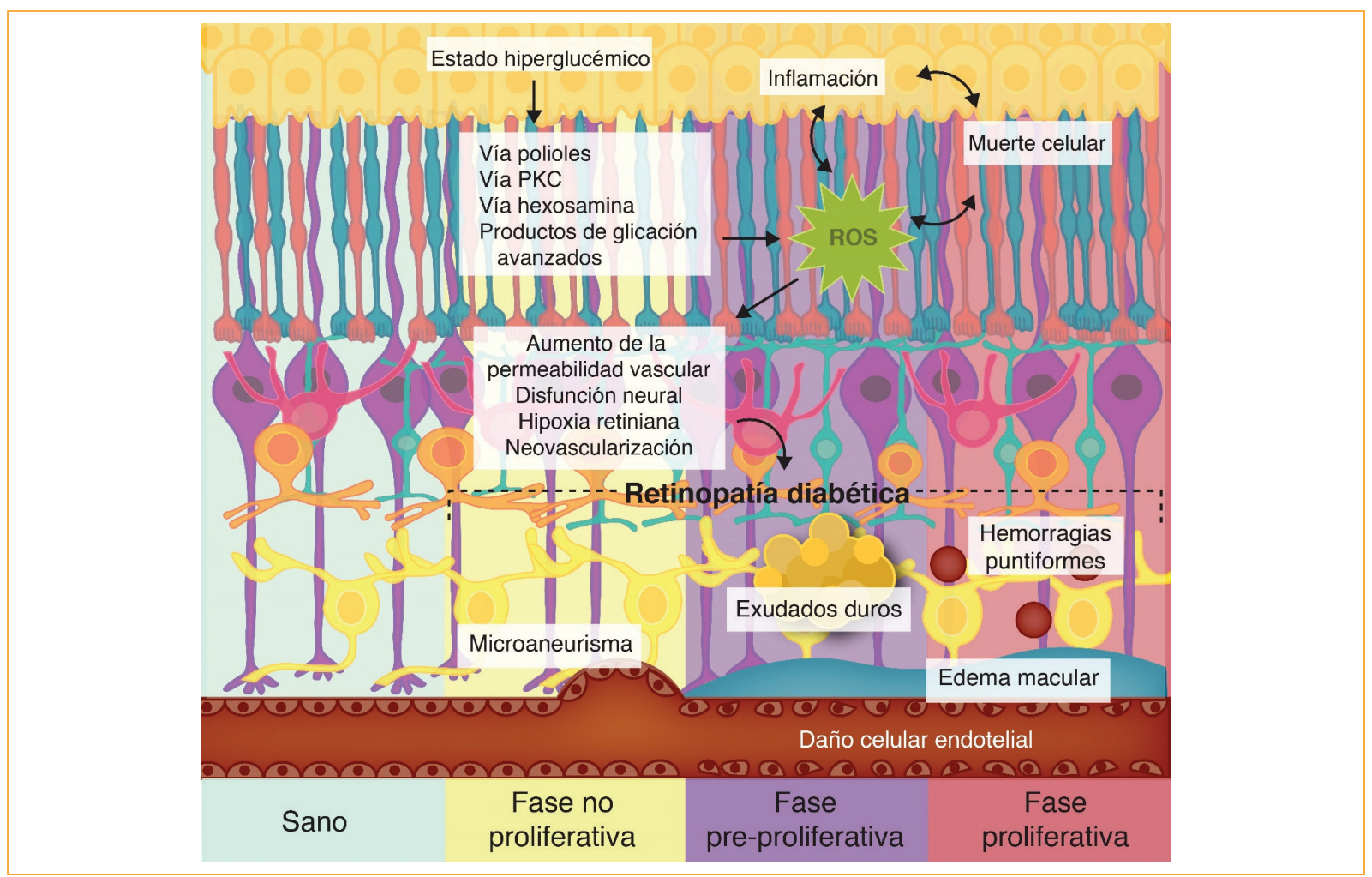

Figura 2. Diferentes mecanismos subyacentes a la retinopatía diabética. (Adaptada de Wu, et al.'1).

factores que influyen en la formación del edema retiniano son la permeabilidad de la BHR, la presión hidrostática capilar, la presión hidrostática tisular, la presión osmótica tisular y la presión osmótica plasmática. El transporte activo por el EPR es necesario para eliminar el agua que se filtra a través de la retina de la presión intraocular, y también es un mecanismo de seguridad contra la acumulación de líquido en la enfermedad.

En 1984, Cunha-Vaz y Travas propusieron que el EMD se podría explicar por la ley de Starling, que explica que la "fuerza» que conduce el agua a través de la pared capilar es el resultado de una diferencia de presión hidrostática y una diferencia de presión osmótica efectiva.

\section{Factor de crecimiento vascular endotelial}

La familia VEGF pertenece a un grupo de glucoproteínas diméricas que incluye VEGF-A, VEGF-B, VEGF-C, VEGF-D, VEGF-E, VEGF-F y el factor de crecimiento placentario. El VEGF-A es una citocina potente y multifuncional que ejerce su efecto sobre el endotelio. En esta revisión, cada vez que se menciona el VEGF nos referiremos al VEGF-A ${ }^{4}$.
EI VEGF actúa de manera directa y selectiva a través de los receptores VEGFR-1 y VEGFR-2, expresados predominantemente por el endotelio vascular. La unión del VEGF a estos receptores provoca una afluencia de calcio citoplasmático, aumentando su concentración hasta cuatro veces, con cambio de forma, división y migración celular. Este aumento en la permeabilidad de las vénulas a las macromoléculas permite que las proteínas plasmáticas se filtren en el espacio extravascular, lo que conduce a la coagulación del fibrinógeno y al depósito de gel de fibrina, que sirve como matriz temporal para el crecimiento de nuevos vasos sanguíneos. El aumento de la permeabilidad microvascular parece preceder o acompañar invariablemente a la angiogénesis en una variedad de procesos fisiológicos y patológicos.

EI VEGF-A puede ser producido por numerosos tipos de células de la retina (células de Müller, células endoteliales, EPR y astrocitos), y la expresión de VEGF-A aumenta en estas células en respuesta a la hiperglucemia, la hipoxia (a través del factor inducible por hipoxia 1 alfa), la estimulación de AGE y citocinas inflamatorias como IL-1 $\beta^{4}$.

EI VEGF-A ejerce efectos proproliferativos y propermeabilidad en las células endoteliales vasculares al 


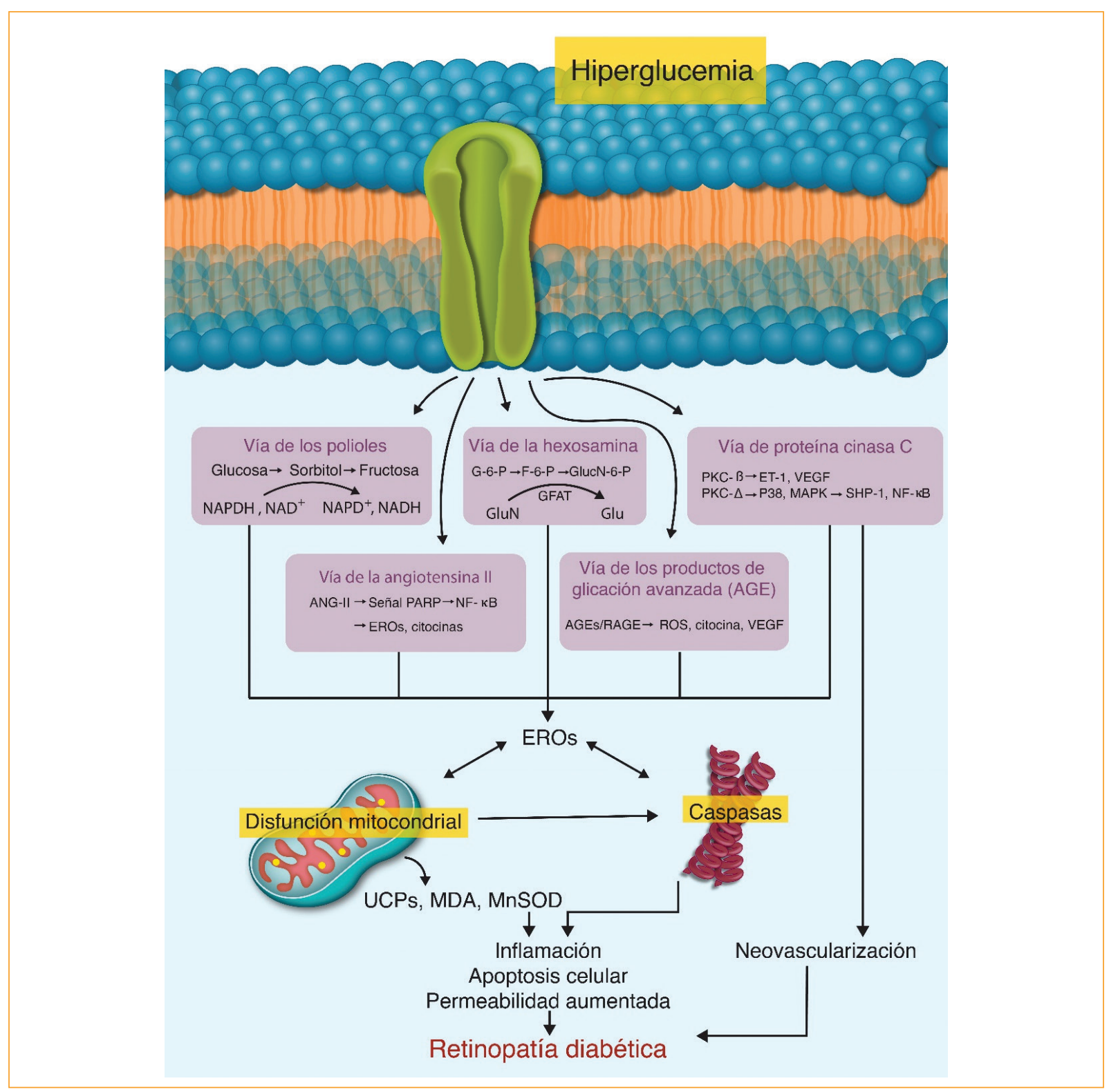

Figura 3. Mecanismos subyacentes al aumento del estrés oxidativo inducido por hiperglucemia involucrado en la patogénesis de la retinopatía diabética. (Adaptada de $\mathrm{Wu}$, et al. ${ }^{1}$ ).

unirse al VEGF-R2. Tras la activación del VEGF-R2, la actividad tirosina cinasa del receptor aumenta, lo que resulta en la activación de múltiples vías de señalización, incluyendo la fosfatidilinositol-3-cinasa Akt, la PKC, la óxido nítrico sintetasa endotelial y proteínas cinasas activadas por mitógeno/cinasas reguladas por señal extracelular. Para inducir una mayor permeabilidad vascular, la PKC activada por el VEGF-A fosforila los componentes ocludina y cadherina de las uniones estrechas y adherentes, respectivamente, lo que resulta en su translocación de la barrera célula-célula a los compartimentos intracelulares. En consecuencia, la BHR se ve comprometida, lo que resulta un mecanismo esencial para el desarrollo del EMD. Además, el VEGF-A tiene un efecto mitogénico directo sobre las células endoteliales vasculares y promueve la alteración de la matriz extracelular requerida para los procesos neovasculares mediante la estimulación de la expresión de metaloproteasas de la matriz. Es probable que ambos mecanismos regulados por el VEGF-A sean importantes para el desarrollo de retinopatía diabética proliferativa. Finalmente, el VEGF-A también tiene otras funciones fisiopatológicos en la inducción de la leucostasis a través de la regulación positiva de la expresión de ICAM-15-9. 


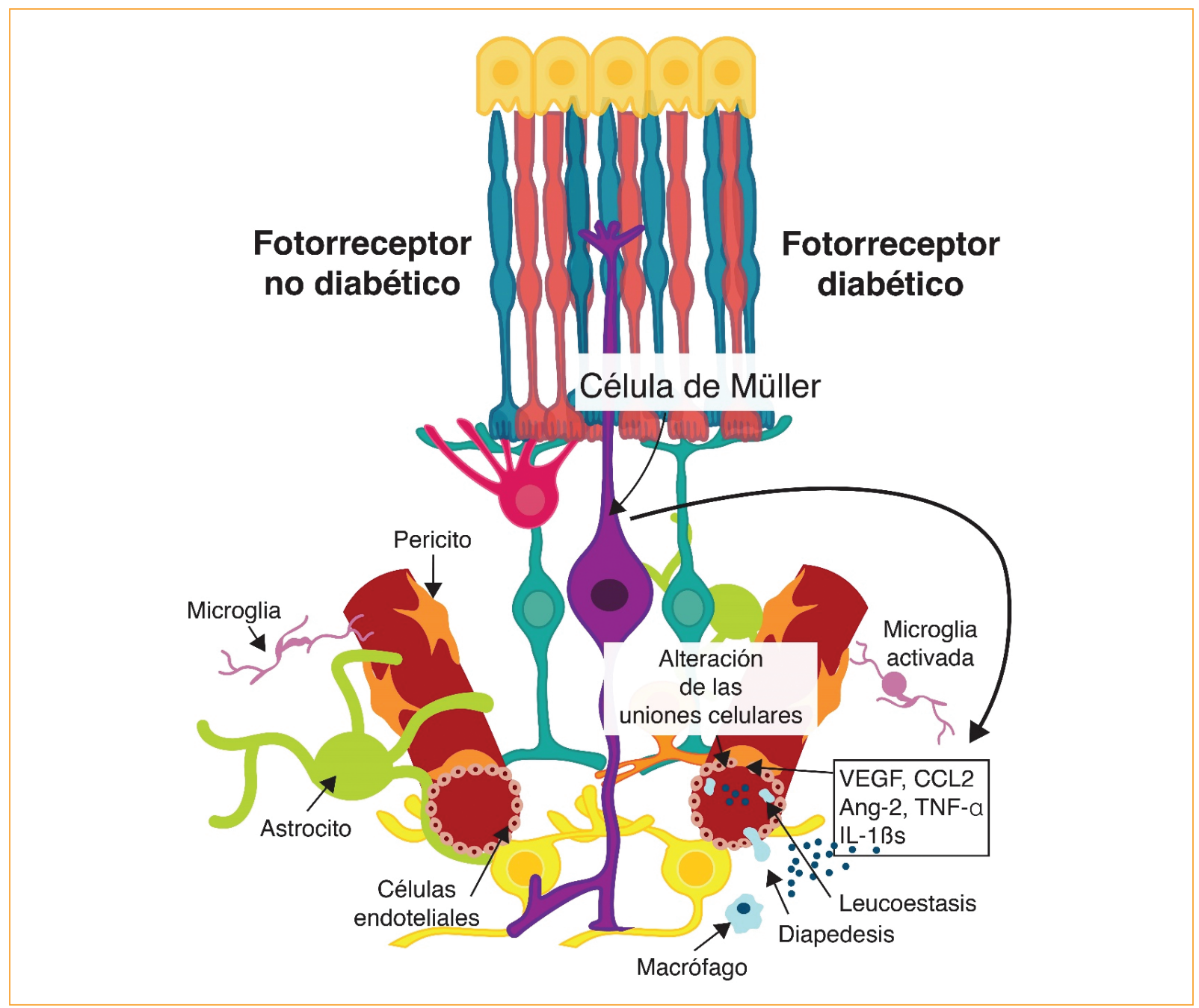

Figura 4. Unidad neurovascular de la retina en condiciones diabéticas y no diabéticas. (Adaptada de Das, et al. ${ }^{2}$ ).

El VEGF es un potente mediador inflamatorio, así como un notorio agente proangiogénico. Curiosamente, la expresión de VEGF está regulada por la ciclooxigenasa 2 y el factor de transcripción kB (NF-kB). Por lo tanto, la inflamación regula las principales alteraciones patológicas posteriores, a saber, la angiogénesis y la apoptosis de las células y las neuronas endoteliales de la retina, lo que conduce a una marcada progresión de la retinopatía diabética.

\section{Bibliografía}

1. Wu M, Yiang G, Lai T, Li C. The oxidative stress and mitochondrial dysfunction during the pathogenesis of diabetic retinopathy. Oxid Med Cel Long. 2018;2018:3420187.
2. Das A, McGuire $P$, Rangasamy S. Diabetic macular edema: pathophysiology and novel therapeutic targets. Ophthalmology. 2015;122:1375-94.

3. Tan G, Cheung N, Simó R, Cheung G, Wong T. Diabetic macular oedema. Lancet Diabetes Endocrinol. 2017;5:143-55.

4. Lally D, Shah C, Heier J. Vascular endothelial growth factor and diabetic macular edema. Surv Ophthalmol. 2016;71:759-68.

5. Whitehead M, Osborne A, Widdowson P, Man Y, Martin K. Angiopoietins in diabetic retinopathy: current understanding and therapeutic potential. J Diabet Res. 2019;2019:5140521.

6. Whitehead M, Wickremasinghe S, Osborne A, Van Wijngaarden P, Martin R. Diabetic retinopathy: a complex pathophysiology requiring novel therapeutic strategies. Expert Opin Biol Ther. 2018;18:1257-70.

7. Safi S, Qvist R, Kumar S, Batumalaie K, Ismail S. Molecular mechanisms of diabetic retinopathy, general preventive strategies and novel therapeutic targets. Biomed Res Int. 2014;2014:801269.

8. Wang W, Lo Y. Diabetic retinopathy: pathophysiology and treatments. Int J Mol Sci. 2018;19:1816.

9. Kusuhara S, Fukushima Y, Ogura S, Inoue N, Uemura A. Pathophysiology of diabetic retinopathy: the old and the new. Diabetes Metab J. 2018;42:364-76 


\title{
Edema macular diabético: enfermedad inflamatoria vascular mediada por múltiples moléculas proinflamatorias
}

\author{
Patricio Rodríguez-Valdéz* \\ Instituto de Oftalmología y Ciencias Visuales, Tecnológico de Monterrey, Monterrey, Nuevo León, México
}

Si bien el factor de crecimiento endotelial vascular (VEGF) desempeña un papel muy importante en la fisiopatología del edema macular diabético (EMD), el proceso es complejo e involucra múltiples vías biológicas en la retina, principalmente a través de citocinas inflamatorias, muchas de la cuales no son comprendidas en su totalidad. Por mencionar un ejemplo, sabemos que alrededor del $30 \%$ de los pacientes tratados con anti-VEGF son resistentes a la terapia antiangiogénica, por lo cual otras citocinas pueden estar también implicadas en el EMD, y que presentan una respuesta favorable al tratamiento ulterior con esteroides intravítreos, lo que pudiera ser explicado por un proceso inflamatorio no dependiente del VEGF. Figueras-Roca, et al. ${ }^{4}$ han analizado meticulosamente la relación entre biomarcadores tomográficos y biomarcadores inflamatorios en el acuoso (citocinas), y han encontrado diferencias en los patrones entre diferentes pacientes con EMD; es decir, los procesos que llevan al EMD no son los mismos en todos los pacientes ${ }^{1-5}$.

\section{Hiperglucemia}

\section{Productos glucosilados avanzados}

Sabemos que la hiperglucemia crónica produce una glucosilación enzimática de las proteínas, dejando como resultado productos glucosilados avanzados (AGE, advanced glycation end products). Estos AGE modifican la matriz extracelular y producen rigidez vascular, pero aún más importante es que estimulan al receptor de RAGE en la membrana endotelial, activando diversas vías metabólicas que aumentan la expresión de citocinas inflamatorias, como la molécula de adhesión intercelular 1 y el VEGF, así como la disminución del óxido nítrico, lo cual produce estrés oxidativo que daña los pericitos y favorece la permeabilidad vascular ${ }^{6,7}$.

\section{Vía del poliol}

La aldosa reductasa transforma el excedente de glucosa en sorbitol, y en un segundo paso el sorbitol es transformado en fructosa y disminuye los niveles de glutatión, el cual es un antioxidante. La acumulación de sorbitol y la disminución de glutatión intracelulares tiene como resultado final el daño celular ${ }^{6,7}$.

\section{Activación de la proteína cinasa C}

El aumento de diacilglicerol activa a la enzima proteína cinasa $\mathrm{C}$, la cual a su vez produce un aumento en la expresión de múltiples citocinas, como el VEGF y el factor de crecimiento transformante beta-1, las cuales tienen como resultado final la acumulación en la matriz extracelular, la fibrinólisis y la inflamación ${ }^{6,7}$.

\section{Radicales libres de oxígeno}

El estrés oxidativo por acúmulo de radicales libres de oxígeno (ROS) se ha relacionado con el engrosamiento de la membrana basal y la pérdida de pericitos. La acumulación de AGE aumenta la cantidad de ROS, lo cual, sumado 


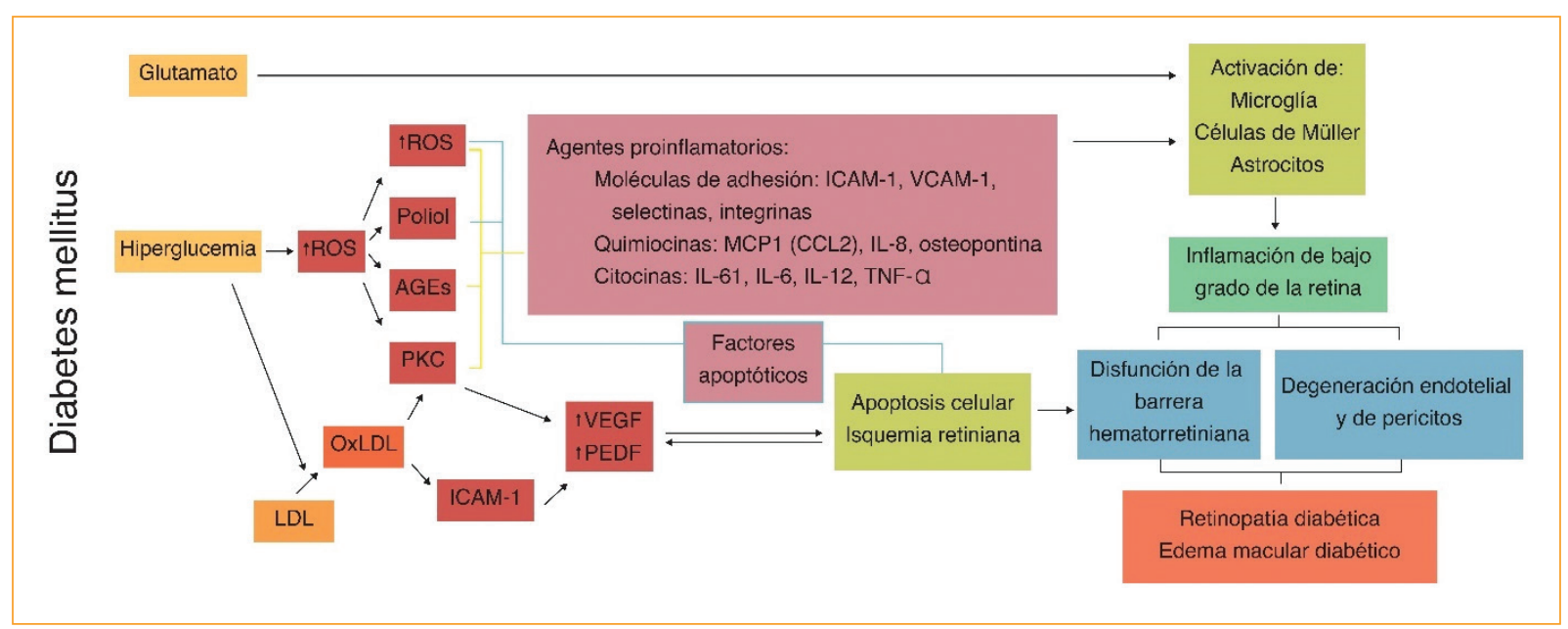

Figura 1. Diferentes vías metabólicas en la hiperglucemia que llevan al proceso inflamatorio en la retina. (Adaptada de Romero, et al. ${ }^{7}$.

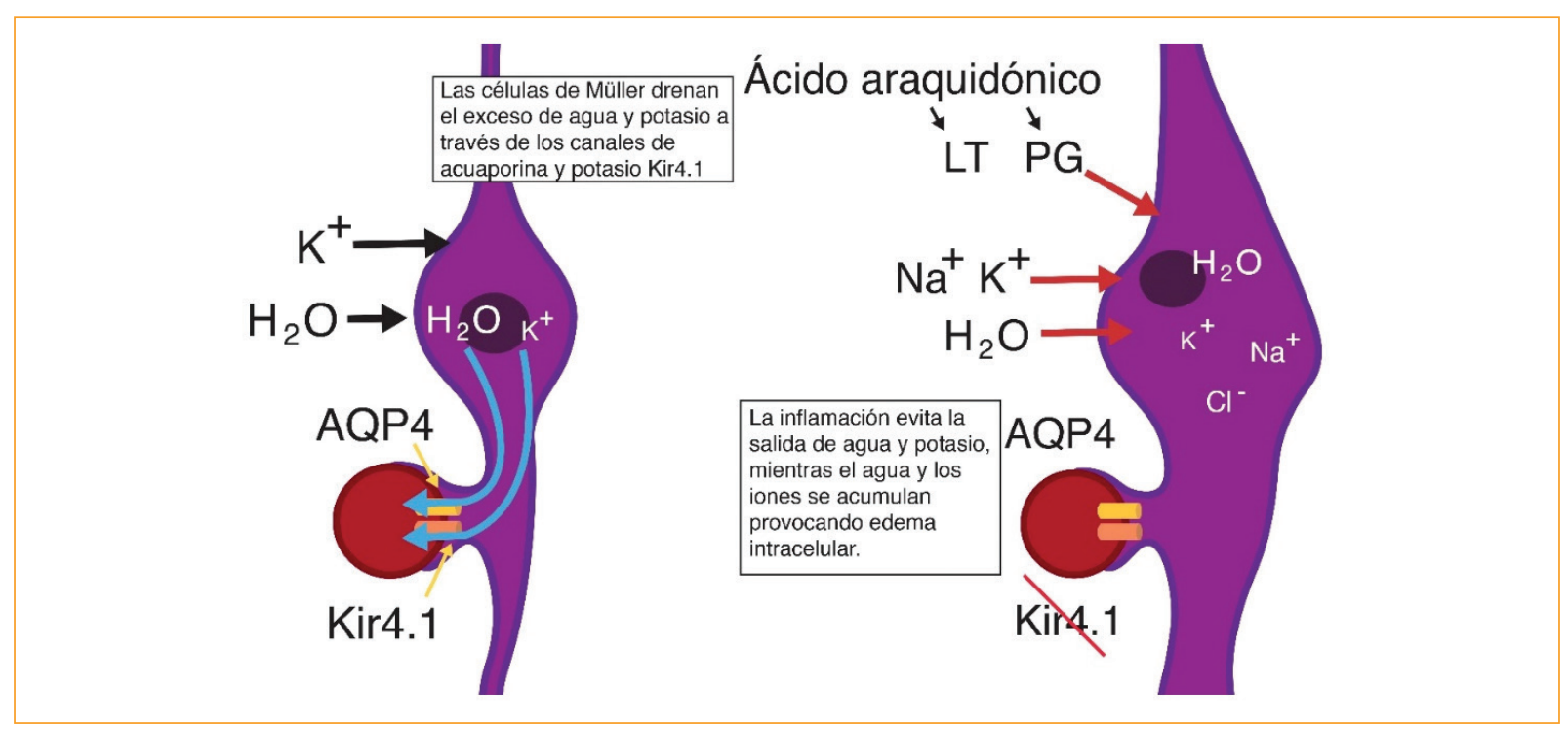

Figura 2. Edema citotóxico secundario a la disfunción de los canales de agua y potasio (acuaporina 4 y Kir 4). (Adaptada de Reichenbach, et al. ${ }^{9}$ ).

a la disminución del glutatión (antioxidante) por la activación de la vía del poliol, produce daño celular e inflamación, y por si fuera poco, la presencia de ROS activa a su vez la proteína cinasa $\mathrm{C}$. En la figura 1 se muestra la interacción de las cuatro vías que tiene como resultado un proceso inflamatorio en la retina diabética ${ }^{6,7}$.

\section{Patogénesis}

La acumulación de líquido intracelular se conoce como edema citotóxico, mientras que la acumulación de líquido extracelular se conoce como edema vasogénico.
Estas dos formas están presentes en los pacientes con EMD, iniciando con el edema citotóxico y apareciendo el edema vasogénico en etapas más tardías ${ }^{8,9}$.

El edema citotóxico se produce por la acumulación de sorbitol, fosfato y lactato intracelular secundario a la hiperglucemia, mientras que el edema vasogénico está mediado principalmente por citocinas inflamatorias, ROS, óxido nitroso y VEGF. El resultado final del edema vasogénico es la acumulación de líquido principalmente en la capa plexiforme externa de la retina. Las células de Müller son las principales encargadas 


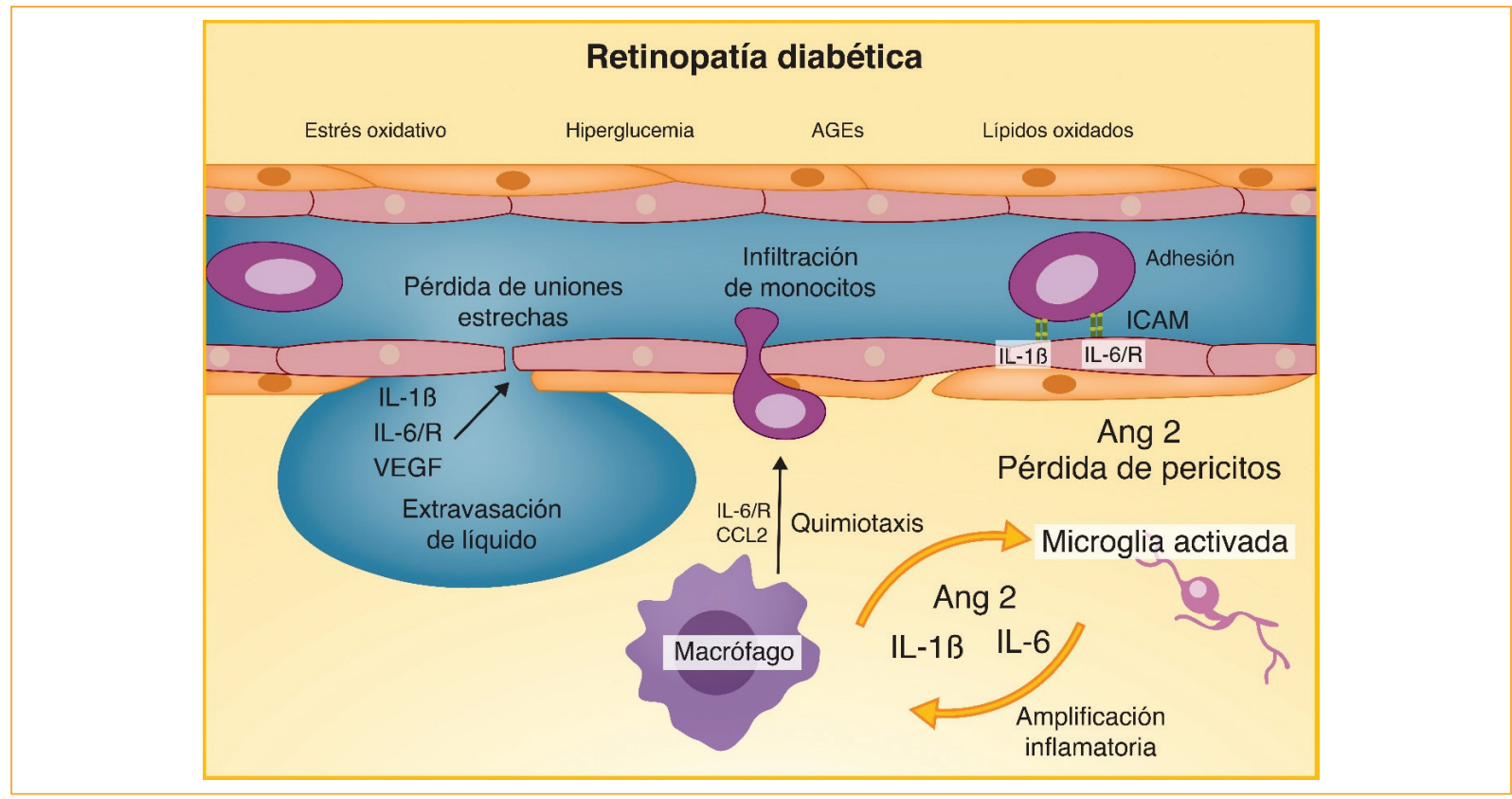

Figura 3. Células y citocinas inflamatorias en la retina diabética.

Tabla 1. Algunas citocinas involucradas en la patogénesis del edema macular diabético y sus funciones

\begin{tabular}{|c|c|c|}
\hline Factor & Abreviatura & Relevancia clínica \\
\hline Angiopoyetina 1 y 2 & Ang 1/Ang2 & Angiogénesis y neovascularización \\
\hline Eritropoyetina & Epo & Estimula la proliferación celular endotelial retiniana \\
\hline Factor de crecimiento hepatocitario & HGF & $\begin{array}{l}\text { Estimula la proliferación, migración e invasión de células retinianas } \\
\text { endoteliales }\end{array}$ \\
\hline Proteína $\mathrm{C}$ reactiva de alta sensibilidad & HsCRP & Probable asociación con EMCS y exudados duros \\
\hline $\begin{array}{l}\text { Factor de crecimiento semejante a la } \\
\text { insulina tipo } 1\end{array}$ & IGF-1 & Angiogénesis \\
\hline Molécula de adhesión intercelular 1 & ICAM-1 & Probable asociación con EMCS y exudados duros \\
\hline Interleucina 6 & IL-6 & Permeabilidad vascular \\
\hline Interleucina B & IL-B & $\begin{array}{l}\text { Mecanismo desconocido, aumentado en EMD, pero no en edema por } \\
\text { enfermedad vascular oclusiva }\end{array}$ \\
\hline Proteína quimiotáctica de monocitos 1 & MCP-1 & Leucostasis que lleva a hipoxia \\
\hline Factor derivado de epitelio pigmentario & PEDF & Antiangiogénico y antiinflamatorio \\
\hline Proteína cinasa C & PKC & Aumenta la permeabilidad vascular y contractilidad \\
\hline Factor derivado de estroma 1 & SDF-1 & Angiogénesis \\
\hline Tromboespondinas 1 y 2 & TSP-1 y 2 & Antiangiogénico: inhibe la proliferación celular endotelial y apoptosis \\
\hline Factor de crecimiento endotelial vascular & VEGF & Angiogénesis y permeabilidad vascular \\
\hline
\end{tabular}

de la homeostasis extracelular de la retina y resultan dañadas por los mecanismos antes mencionados, de manera tal que su falla permite la acumulación de líquido extracelular y la aparición clínica del EMD. Se cree que la disfunción de las células de Müller está causada por una afectación de las acuaporinas 4 y Kir4 
(canales de agua y potasio en su membrana), y que al alterarse su función permiten el edema intracelular (citotóxico) y la subsecuente disfunción celular (figura 2). Los esteroides tienen efectos de restauración de estos canales y promueven la homeostasis 8,9 .

Otro proceso que resulta de la actividad de las diferentes vías patológicas en la retina diabética es la activación de las células de la microglía y los astrocitos, que junto con las células de Müller inician el proceso de inflamación mediada por las interleucinas 6 y 8 . La interleucina 6 afecta a los astrocitos que dan soporte a la vasculatura retiniana, con la consecuente disrupción de la barrera hematorretiniana interna. La interleucina 8 atrae neutrófilos y monocitos. La figura 3 muestra la interacción de estos mecanismos celulares ${ }^{9}$.

Muchas otras citocinas inflamatorias desempeñan un papel en la patogénesis del EMD. La tabla 1 muestra algunas de ellas, con sus respectivas funciones conocidas ${ }^{9}$.

En conclusión, el EMD está causado por un proceso inflamatorio complejo que altera la homeostasis, y no solo por la producción de VEGF como respuesta a la hipoxia.

\section{Bibliografía}

1. Wells J, Glassman A, Ayala A, Jampol L, Aiello L, Antoszyk A, et al. Aflibercept, bevacizumab or ranibizumab for diabetic macular edema. New Engl J Med. 2015;372:1193-203.

2. Busch C, Zur D, Fraser S, Laíns I, Santos A, Lupidi M, et al. Shall we stay, or shall we switch? Continued anti-VEGF therapy versus early switch to dexamethasone implant in refractory diabetic macular edema. Acta Diabetologica. 2018;55:789-96

3. Busch C, Fraser S, Iglicki M, Lupidi M, Couturier A, Chaikitmongkol V, et al. Real-world outcomes of non-responding diabetic macular edema treated with continued anti-VEGF therapy versus early switch to dexamethasone implant: 2-year results. Acta Diabetologica. 2019;56:1341-50.

4. Figueras M, Sala A, Alforja S, Torras J, Peraza J, Zarranz J, et al Aqueous humour cytokine changes with intravitreal dexamethasone implant injection for diabetic macular edema. Ocular Immunol Inflam. 2019;27:1203-10.

5. Figueras M, Sala A, Zarranz J, Alba C, Alforja S, Esquinas C, et al. Anatomic response to intravitreal dexamethasone implant and baseline aqueous humor cytokine levels in diabetic macular edema. Invest Ophthalmol Vis Sci. 2019;60:1336-43.

6. Mesquida, M, Drawnel F, Fauser S. The role of inflammation in diabetic eye disease. Semin Immunopathol. 2019;41:427-45.

7. Romero P, Baget M, Pareja A, López M, Navarro R, Verges R. Diabetic macular edema pathophysiology: vasogenic versus inflammatory. J Diab Res 2016;2016:2156273.

8. Chung Y, Kim Y, Ha S, Byeon H, Cho C, Kim J, et al. Role of inflammation in classification of diabetic macular edema by optical coherence tomography. J Diab Res. 2019;2019:8164250.

9. Reichenbach A, Wurm A, Pannicke T, landiev I, Wiedemann P, Bringmann A. Muller cells as players in retinal degeneration and edema. Graefe's Arch Clin Experiment Ophthalmol. 2007;245:627-36. 


\title{
Biomarcadores pronósticos y predictivos oculares y sistémicos del edema macular diabético
}

\author{
Ximena Mira-Lorenzo* \\ Centro Oftalmológico Arcos, Santiago de Querétaro, Querétaro, México
}

Como es del conocimiento general, la diabetes es un gran problema crónico de salud caracterizado por la falta absoluta o relativa de insulina. Es muy importante identificar a los pacientes que tengan mayor riesgo de desarrollar edema macular diabético (EMD) y pérdida visual para entender sus causas y su desarrollo, y ofrecerles un apropiado tratamiento para evitar la pérdida de la visión por EMD.

Las enfermedades crónicas degenerativas en etapas tempranas inician con una fase libre de síntomas. Durante esta etapa puede haber mayor o menor probabilidad de desarrollar síntomas. En estos casos, los biomarcadores permiten identificar a individuos con alto riesgo de forma confiable y a tiempo para que puedan ser tratados.

Los biomarcadores se han convertido en la base de la medicina preventiva, pues permiten reconocer enfermedades 0 un riesgo temprano de estas, para así tomar las medidas necesarias y evitar el desarrollo de enfermedades o de sus complicaciones ${ }^{1}$.

El National Institute of Health estableció la definición de biomarcadores como aquellos parámetros biológicos, químicos, antropométricos o fisiológicos que son objetivamente medibles, capaces de identificar procesos fisiológicos o patológicos, o bien una respuesta farmacológica a una intervención terapéutica. Existen biomarcadores pronósticos y predictivos ${ }^{1}$ :

- Los biomarcadores pronósticos indican la progresión de la enfermedad con y sin tratamiento.

- Los biomarcadores predictivos permiten conocer una respuesta esperada ante un tratamiento en particular.
Para usar un biomarcador en el diagnóstico del EMD, el procedimiento para obtenerlo debe ser sencillo y fácilmente aceptado por el paciente. Una prueba rápida que entregue un resultado en minutos sería lo óptimo. Esto permite que el médico tenga una comunicación clara y objetiva para una eficaz toma de decisiones en el manejo de la enfermedad. Existen biomarcadores sistémicos y oculares ${ }^{1}$.

\section{Biomarcadores sistémicos}

\section{BIOMARCADORES SÉRICOS}

La relación entre un control intensivo de la diabetes y la prevención de la progresión de la retinopatía diabética se estableció en el Diabetes Control and Complications Clinical Trial/Epidemiology of Diabetes Interventions and Complications Study. Este estudio describió las diferencias de las complicaciones retinianas entre dos grupos con diferente control y esquema de tratamiento (tratamiento convencional frente a tratamiento intensivo) durante 10 años; también encontró que disminuir un $1 \%$ la hemoglobina glucosilada ( $\mathrm{HbA1c}$ ) disminuye la progresión de la retinopatía diabética en un $30-40 \%{ }^{2}$.

Existen múltiples biomarcadores séricos que favorecen la evolución del EMD con el aumento de lipoproteínas de baja densidad que favorecen la apoptosis de los pericitos; la dislipidemia favorece la disfunción endotelial y disminuye la biodisponibilidad del óxido nítrico ${ }^{3}$. 


\section{Hemoglobina gLUCOSILADA COMO BIOMARCADOR PRONÓSTICO}

Existen múltiples biomarcadores sistémicos que se han asociado con retinopatía diabética y EMD, como la proteína $C$ reactiva, productos de glicación final y lípidos séricos, pero la $\mathrm{HbA1c}$ es el único biomarcador válido y estadísticamente significativo ${ }^{4}$.

En los pacientes diabéticos, la hiperglucemia es un gran detonador para daño tisular, como el daño capilar a las células endoteliales de la retina y la rotura de la barrera hematorretiniana.

Sabemos que el control intensivo de la glucosa ha probado ser efectivo para disminuir la incidencia de desarrollo y progresión de retinopatía diabética en tipo 1 y 3 en los estudios DCCT y UKPDS ${ }^{3}$.

El EMD se define por tomografía de coherencia óptica (OCT) como un grosor macular central de $300 \mu \mathrm{m}$ o más, con presencia de quistes intrarretinianos y líquido subretiniano. Otros parámetros que se han incluido son el volumen foveal y el volumen macular total. El grosor macular central y el volumen foveal se calculan dentro de $1 \mathrm{~mm}$ central de la rejilla ${ }^{5}$.

\section{Hemoglobina glucosilada COMO biomarcador PREDICTIVO}

En el análisis exploratorio del protocolo T se describió que, por cada $1 \%$ de aumento de la $\mathrm{HbA} 1 \mathrm{c}$, la agudeza visual en 2 años disminuye 0.5 letras, y se observó que a menor HbA1c había mayor ganancia de letras a 2 años con cualquiera de los tres antiangiogénicos utilizados en el estudio (tabla 1$)^{6}$.

\section{Edad}

Las diferentes características basales que tienen los pacientes de forma demográfica, así como los hallazgos oculares, han sido evaluados para estudiar la evolución y la respuesta al tratamiento del edema macular diabético, incluyendo la edad.

\section{BIOMARCADOR PRONÓSTICO}

El Diabetic Retinopathy Clinical Reasearch Network (DRCR.net) describió que los pacientes con una edad igual o menor de 50 años tienen la habilidad de tolerar más el fluido sin incurrir en pérdida potencial de la visión. El estudio realizado por Chou, et al. ${ }^{5}$ describe una relación directa en pacientes menores de 50 años que tienen mayor grosor macular central, por lo que
Tabla 1. Ganancia de letras con varios antiangiogénicos con diferentes valores de hemoglobina glucosilada

\begin{tabular}{|l|c|c|c|}
\hline HbA1c & $\begin{array}{c}\text { Ganancia de } \\
\text { letras con } \\
\text { aflibercept }\end{array}$ & $\begin{array}{c}\text { Ganancia de } \\
\text { letras con } \\
\text { bevacizumab }\end{array}$ & $\begin{array}{c}\text { Ganancia de } \\
\text { letras con } \\
\text { ranibizumab }\end{array}$ \\
\hline$<6.5 \%$ & 13.5 & 12.4 & 12.4 \\
\hline $6.5 \%$ a $<7.5 \%$ & 12.6 & 9.7 & 12.9 \\
\hline $7.5 \%$ a $<8.5 \%$ & 14.8 & 8.6 & 13.3 \\
\hline $8.5 \%$ a $<9.5 \%$ & 10.8 & 10.3 & 13.5 \\
\hline$>9.5 \%$ & 11.9 & 10.5 & 9.4 \\
\hline
\end{tabular}

HbA1c: hemoglobina glucosilada. Adaptada del Protocolo T ${ }^{6}$.

describen que hay mayor riesgo de EMD en los menores de 50 años, ya que en una muestra de 102 pacientes, 9 pacientes eran menores de 50 años y 5 de ellos tuvieron un grosor macular central $>325 \mu \mathrm{m}^{5,7,8}$.

Con estos hallazgos debemos monitorizar con frecuencia a los pacientes menores de 50 años, ya que pueden tolerar cambios estructurales en la mácula sin disminución de la visión y, por lo tanto, tardan más en llegar a una revisión oftalmológica y llegan años después, cuando ya el daño estructural es mayor y va acompañado de pérdida visual.

\section{BIOMARCADOR PREDICTIVO}

En el Protocolo T, los pacientes jóvenes tuvieron mejor respuesta al tratamiento antiangiogénico intravítreo descrito: 2.1 letras en un periodo de 2 años por cada 10 años de decremento. Esta asociación apoya los hallazgos previos del protocolo I, en el cual hubo un aumento de 2.2 letras por cada 10 años de juventud durante 1 año. En RISE y RIDE, en el análisis de 502 ojos se describió que por cada 5 años que aumenta el paciente de vida la ganancia de 15 letras disminuye a 2 años ${ }^{6}$.

\section{Biomarcadores genéticos}

\section{Pronóstico}

Se ha estudiado la asociación genética y se evidencia la herencia en un $27-52 \%$ de pacientes con EMD, encontrando una correlación con múltiples genes, como ACE, GRB2, AKR1B1, ICAM1, NOS, PPARGC1A y VEGF, pero estadísticamente significativa solo con dos variantes del gen de la molécula de adhesión intercelular (ICAM1), lo cual evidencia y confirma la base inflamatoria en el desarrollo del EMD. Debemos tomar 
en cuenta las modificaciones epigenéticas en el EMD para entender mejor su evolución ${ }^{5}$.

\section{Duración de la diabetes tipo 2}

EI WESDR tiene un gran seguimiento de pacientes con diabetes mellitus tipo 1 por 25 años, y ha descrito una incidencia acumulativa de EMD, en el cual la incidencia tiene una meseta a los 14 años, sumando los últimos 11 años a la incidencia acumulativa de los 25 años. En la diabetes mellitus tipo 2, la información es limitada e inconsistente ${ }^{9}$.

\section{Biomarcadores oculares}

\section{Biomarcadores en el humor acuoso}

La ICAM-1 se ha identificado como un biomarcador crucial en el EMD. Está presente en el humor acuoso de los pacientes con diabetes mellitus tipo 2 y se ha observado una correlación de esta molécula con un aumento del grosor macular central. Los estudios han demostrado que, a más ICAM-1 y menos factor de crecimiento endotelial vascular (VEGF, vascular endothelial growth factor) en el humor acuoso, mejor es la respuesta al antiangiogénico para disminuir el grosor macular central ${ }^{4}$.

\section{Biomarcadores en el vítreo}

EI VEGF tiene un papel importante en el aumento de la permeabilidad vascular. Un estudio encontró que esta molécula es estadísticamente significativa para el desarrollo de EMD. El factor derivado del epitelio pigmentado se encuentra disminuido en el vítreo de los pacientes con EMD ${ }^{4,10}$.

\section{Agudeza visual}

La agudeza visual se toma como un biomarcador pronóstico y predictivo. Los pacientes que ingresan con agudezas visuales pobres, menores de 20/50, tienen mayor grosor macular central, pero tienen más oportunidad de una mayor ganancia de letras alcanzando como máximo 20/30. Como biomarcador predictivo está descrito por el protocolo $\mathrm{T}$ que, en los pacientes con agudeza visual 20/32 a 20/40, la agudeza visual mejora 7.8 letras con aflibercept, 8.6 letras con ranibizumab y 6.8 letras con bevacizumab. En los pacientes con agudeza visual de 20/50 a 20/320, la agudeza visual mejora 18.1 letras con aflibercept, 16.1 letras con ranibizumab y 13.3 letras con bevacizumab ${ }^{8,11}$.

\section{Cambio en los microaneurismas (turnover): aparición y desaparición}

A través de fotografías del fondo de ojo y un software llamado RetmarkerRD se han estudiado el conteo, la aparición y la desaparición de microaneurismas para valorar el desarrollo de EMD. La desaparición de microaneurismas no se debe valorar o tomar como un proceso de reversión, sino que indica daño vascular progresivo y cierre de capilares. Por lo anterior, se debe monitorear y contar la aparición y la desaparición de los microaneurismas en el mismo o en un lugar nuevo; a esto se le llama turnover. La presencia y el número de microaneurismas, así como su rango de aparición y desaparición, son indicadores de progresión de retinopatía diabética, y cuando hay un turnover de al menos dos microaneurismas por año estos pacientes tienen mayor probabilidad de desarrollar EMD (un 70.6\%). Este resultado se confirma con el uso del programa RetmarkerDR. El turnover de microaneurismas tiene un alto índice de predicción para el desarrollo de EMD'.

\section{Edema macular subclínico}

El término "edema macular subclínico» fue propuesto para definir una etapa muy temprana del EMD por Bressler, Browning y Fraser. El DRCR.net demostró que del $25 \%$ al $50 \%$ de los pacientes con edema macular subclínico progresarán a EMD dentro los primeros 2 años posteriores a su identificación. La definición de edema macular subclínico propuesta por el DRCR.net consiste en la ausencia de edema macular que involucre el centro de la fóvea y un grosor macular $>225 \mu \mathrm{m}$ y $<299 \mu \mathrm{m}$. El edema macular subclínico está presente en el $38 \%$ de los casos y tiene un riesgo de 3.686 veces de aumentar la progresión a EMD dentro los 2 primeros años desde la detección.

\section{Grosor macular central}

El grosor macular es un biomarcador predictivo en el cual los anti-VEGF han demostrado su eficacia para disminuirlo. Aunque la efectividad de los anti-VEGF está confirmada, no todos los pacientes responden de igual manera para tener una mejor agudeza visual corregida. En el estudio de Cunha, et al. ${ }^{1}$ se demostró que una reducción $>20 \%$ del grosor macular central mejoró $10.3+13.0$ letras, y cuando la reducción 
del grosor fue $<20 \%$ solo hubo una mejor agudeza visual corregida de $1.8+7.2$ letras.

\section{Angiografía por tomografía de coherencia óptica como biomarcador predictivo}

Lee, et al. ${ }^{9}$ compararon los hallazgos de angio-OCT en pacientes con EMD que respondieron o no al tratamiento con antiangiogénicos. En los pacientes no respondedores y pobres respondedores encontraron un número mayor de microaneurismas, menor flujo vascular y un aumento de la zona avascular foveal en el plexo vascular profundo. Este plexo se encuentra ubicado en la capa plexiforme externa, la cual también muestra pérdida de continuidad y quistes intrarretinianos en los pobres respondedores. En los pacientes con un plexo vascular profundo intacto, también la capa plexiforme externa está continua e intacta, con una buena respuesta al tratamiento antiangiogénico. No hay recuperación ni continuidad de la disrupción de la capa plexiforme externa posterior al tratamiento, lo que pudiera significar un daño microvascular y estructural permanente. Una explicación posible es que los quistes intrarretinianos tengan un contenido proteico que no permita el paso del antiangiogénico del vítreo al plexo vascular profundo, y una segunda explicación es que al existir isquemia del plexo vascular profundo hay mayor expresión de VEGF y esta isquemia restringiría la efectividad del tratamiento antiangiogénico (figura 1) ${ }^{12}$.

\section{Grosor macular coroideo}

La coroides es parte importante en la homeostasis de la retina externa y también en diversas patologías retinianas, como la retinopatía diabética. Se han descrito aumento de la tortuosidad vascular, hemorragia extravascular, microaneurismas, vasodilatación y estenosis, así como neovascularización en los vasos coroideos. Con la llegada de la imagen multimodal llegó la imagen de profundidad mejorada (enhanced depth imaging) en la OCT que permite valorar con detalle las tres capas de la coroides: coriocapilar, capa vascular media (Sattler) y vasos coroideos grandes (Haller). El grosor coroideo promedio en el grupo control fue de $254 \mu \mathrm{m}$, en los pacientes con EMD tipo espongiforme fue de $294+109 \mu \mathrm{m}$, en aquellos con EMD cistoideo fue de $268+70 \mu \mathrm{m}$ y en los paciente con desprendimiento de retina seroso fue de $294+70 \mu \mathrm{m}^{13}$.
Tabla 2. Grosor macular en pacientes con diabetes sin y con retinopatía diabética, y en sujetos control

\begin{tabular}{|l|c|c|c|c|c|}
\hline Grupo & $\begin{array}{c}1 \\
\text { semana }\end{array}$ & $\begin{array}{c}1 \\
\text { mes }\end{array}$ & $\begin{array}{c}3 \\
\text { meses }\end{array}$ & $\begin{array}{c}6 \\
\text { meses }\end{array}$ & $\begin{array}{c}1 \\
\text { año }\end{array}$ \\
\hline Control & $69.8 \%$ & $46.5 \%$ & $41.9 \%$ & $39.5 \%$ & $32.6 \%$ \\
\hline Sin RD & $65.1 \%$ & $55.8 \%$ & $67.4 \%$ & $34.9 \%$ & $32.6 \%$ \\
\hline Con RD & $83.7 \%$ & $93 \%$ & $97.7 \%$ & $30.2 \%$ & $32.6 \%$ \\
\hline
\end{tabular}

RD: retinopatía diabética.

Se ha visto que el grosor coroideo disminuye posterior al tratamiento con anti-VEGF, y tomando en cuenta esto se observa una dependencia del VEGF en el grosor coroideo. Se ha reportado que muchas condiciones sistémicas, hemodinámicas y fisiológicas tienen influencia en el EMD y el grosor coroideo. No existe una correlación negativa entre la agudeza visual y el aumento del grosor coroideo.

\section{Cirugía de catarata no complicada en retinopatía diabética y edema macular diabético}

Muchos estudios han intentado identificar factores de riesgo que favorezcan el EMD posquirúrgico. Se ha observado aumento del grosor macular en sujetos control y en pacientes con diabetes sin y con retinopatía diabética (tabla 2) ) $^{14,15}$.

Existe un aumento del grosor macular central en los pacientes sin diabetes y sin edema macular al mes 1 de $21 \mu \mathrm{m}$ y al mes 3 de $25.5 \mu \mathrm{m}$.

Los pacientes con EMD preexistente a la cirugía con un grosor de $328 \mu \mathrm{m}$, al mes tuvieron un grosor de $446.6 \mu \mathrm{m}$ y a los 3 meses de $452 \mu \mathrm{m}$. Los pacientes con diabetes sin EMD que iniciaron con un grosor preoperatorio de $243.5 \mu \mathrm{m}$, al mes tuvieron $275.7 \mu \mathrm{m}$ $y$ al tercer mes $282 \mu \mathrm{m}^{14}$. Por tanto, todos los pacientes con diabetes operados de facoemulsificación sin EMD tendrán un aumento del grosor macular esperado de aproximadamente $32 \mu \mathrm{m}$ el primer mes y $39 \mu \mathrm{m}$ al tercer mes ${ }^{14}$. Los pacientes con diabetes operados de facoemulsificación con EMD tendrán un aumento de grosor macular esperado de aproximadamente $118 \mu \mathrm{m}$ el primer mes y $124 \mu \mathrm{m}$ al tercer mes $^{14}$.

Los pacientes con retinopatía diabética leve, moderada o grave no tienen diferencia entre ellos para tener cambios en el grosor macular, y en varios estudios excluyen pacientes con fotocoagulación 

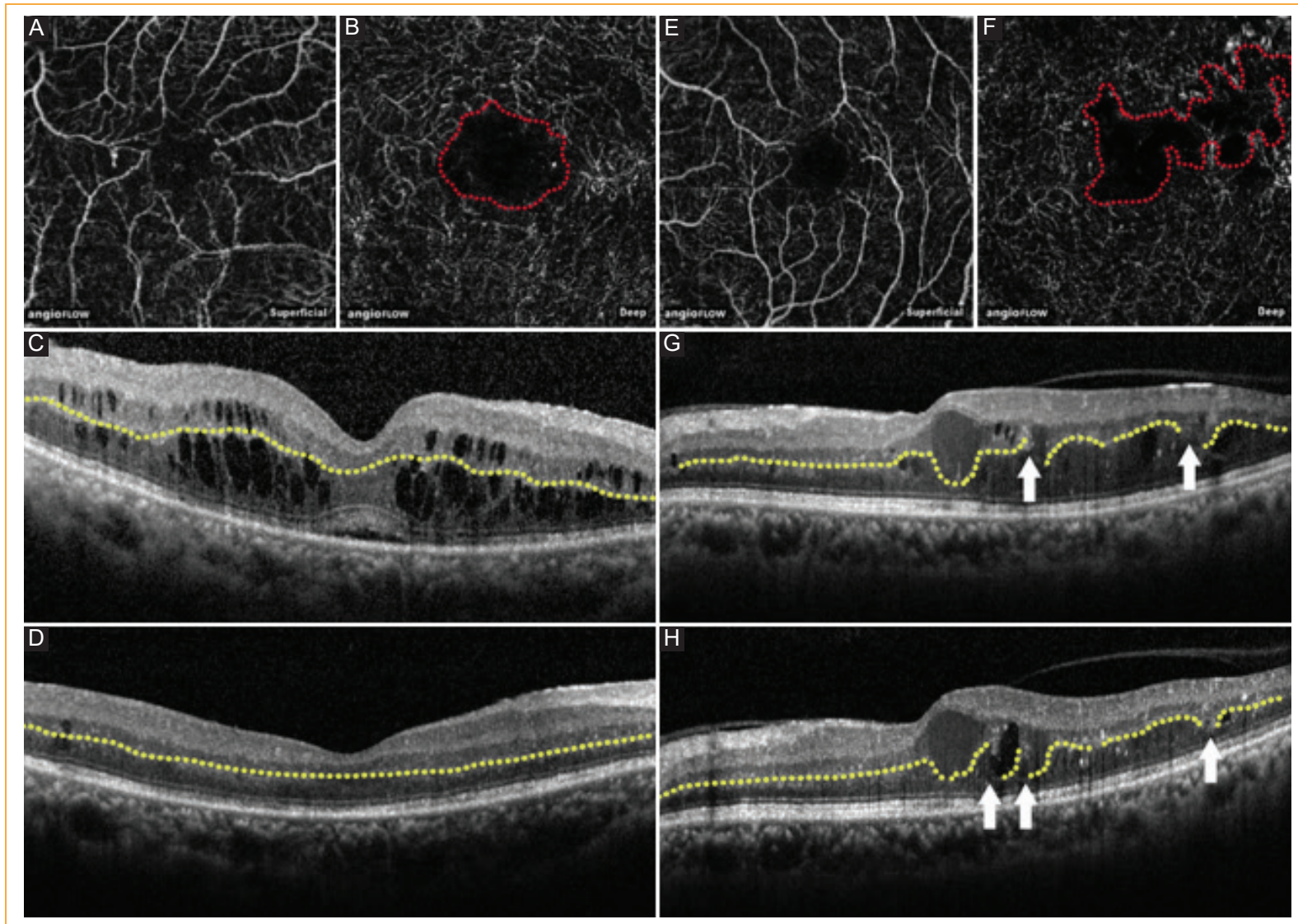

Figura 1. Comparación de la integridad de la capa plexiforme externa de acuerdo con la respuesta a un antiangiogénico. A-D: buen respondedor al tratamiento. E-H: mal respondedor al tratamiento. Se observa una disrupción de la capa plexiforme externa en las imágenes. G-H (líneas punteadas amarillas y flechas). F: área de no perfusión. Las líneas punteadas rojas indican el plexo profundo en la angio-OCT. H: disrupción de la capa plexiforme externa que no se reparó posterior al tratamiento, aunque el edema se resolvió. C-D: se conserva la integridad de la capa plexiforme externa.

panretiniana para eliminar el sesgo de aumento del grosor macular secundario a la fotocoagulación.

\section{Bibliografía}

1. Cunha J, Ribeiro L, Lobo C. Phenotypes and biomarkers of diabetic retinopathy. Prog Retin Eye Res 2014;41:90-111.

2. Dhoot D, Baker K, Saroj N, Vitti R. Baseline factors affecting changes in Diabetic Retinopathy Severity Scale Score after intravitreal aflibercept or laser for diabetic macular edema. Ophthalmology. 2018;125:51-6.

3. Peng Y, Tsai M. Impact of metabolic control on macular thickness in diabetic macular edema. Diab Vasc Dis Res. 2018:15:165-8.

4. Kwan C, Fawzi A. Imaging and biomarkers in diabetic macular edema and diabetic retinopathy. Curr Diab Rep. 2019;19:95

5. Chou T, Wu P, Kuo J, Lai C, Kuo C. Relationship of diabetic macular oedema with glycosylated haemoglobin. Eye. 2009;23:1360-3.

6. Bressler S, Odia I, Maguire M, Dhoot D. Factors associated with visual acuity and central subfield thickness changes when treating diabetic macular edema with antivascular endothelial growth factor therapy. An exploratory analysis of the protocol T-Randomized Clinical Trial. JAMA Ophthalmol. 2019;137:382-9.

7. Raafay S, Na L, Campochiaro P. Predictors of functional and anatomic outcomes in patients with diabetic macular edema treated with ranibizumab. Ophthalmology. 2015;122:1395-401.
8. Bressler S, Haijing Q, Roy W, Beck M. Factors associated with changes in visual acuity and central subfield thickness at 1 year after treatment for diabetic macular edema with ranibizumab. Arch Ophthalmol. 2010;130: 1153-61.

9. Lee R, Wong T, Sabanayagam C. Epidemiology of diabetic retinopathy, diabetic macular edema and related vision loss. Eye Vision. 2015; 2:17

10. McAuley A, Sanfilippo P. Hewitt A. Vitreous biomarkers in diabetic retinopathy: a systematic review and meta-analysis. J Diabet Complic. 2014;28:419-25

11. Wells J, Glassman A, Ayala A, Lee M, Jampol M. Aflibercept, bevacizumab or ranibizumab for diabetic macular edema. Two year results from a comparative effectiveness randomized clinical trial. Ophthalmology. 2016;123:1351-9.

12. Lee J, Moon B, Cho A. Optical coherence tomography angiography of DME and its association with anti-VEGF treatment response. Ophthalmology. 2016;123:2368-75.

13. Endo H, Kase S, Takahashi E, Saito M. Relationship between diabetic macular edema and choroidal layer thickness. PLoS One. 2020;15:e0226630.

14. Chen X, Song W, Cai H. Macular edema after cataract surgery in diabetic eyes evaluated by optical coherence tomography. Int $\mathrm{J}$ Ophthalmol. 2016;9:81-5.

15. Akkaya S, Ozkurt Y. Changes in central macular thickness after uncomplicated phacoemulsification surgery in diabetic and non-diabetic patients. Beyoglu Eye J. 2018;3:13-9. 


\title{
Clasificación del edema macular diabético
}

\author{
Ermilo Sánchez-Buenfil ${ }^{*}$ \\ Centro de Oftalmología Especializada RETIMEDIQ, Mérida, Yucatán, México
}

La necesidad de clasificar y definir el edema macular diabético se remonta a 1968, cuando un grupo de expertos se reunió en Airlie House, Virginia, Estados Unidos de América, y propuso una clasificación cuantitativa y cualitativa para describir el estado de la retinopatía diabética, utilizando fotografías de fondo para registrar lesiones en ciertas áreas predesignadas (figura 1) 1 .

Esta clasificación fue modificada en 1971 para utilizarla en el Diabetic Retinopathy Study, el cual demostró una reducción significativa en el desarrollo de pérdida visual grave en pacientes tratados con fotocoagulación panretiniana en comparación con los no tratados ${ }^{2}$.

La clasificación se desarrolló aún más en el Early Treatment Diabetic Retinopathy Study (ETDRS) (figuras 2 a 5), en el que se evaluó la eficacia del tratamiento con láser para el edema macular. Este se denominó como clínicamente significativo si presentaba cualquiera de las siguientes características ${ }^{3,4}$ :

- Engrosamiento de la retina a $500 \mu \mathrm{m}$ o menos del centro de la mácula.

- Exudados duros a 500 um o menos del centro de la mácula, si están relacionados con el engrosamiento de la retina adyacente.

- Una o varias áreas de engrosamiento retiniano del tamaño de un disco óptico o mayor, teniendo cualquiera de sus partes a la distancia de un disco óptico del centro de la mácula.

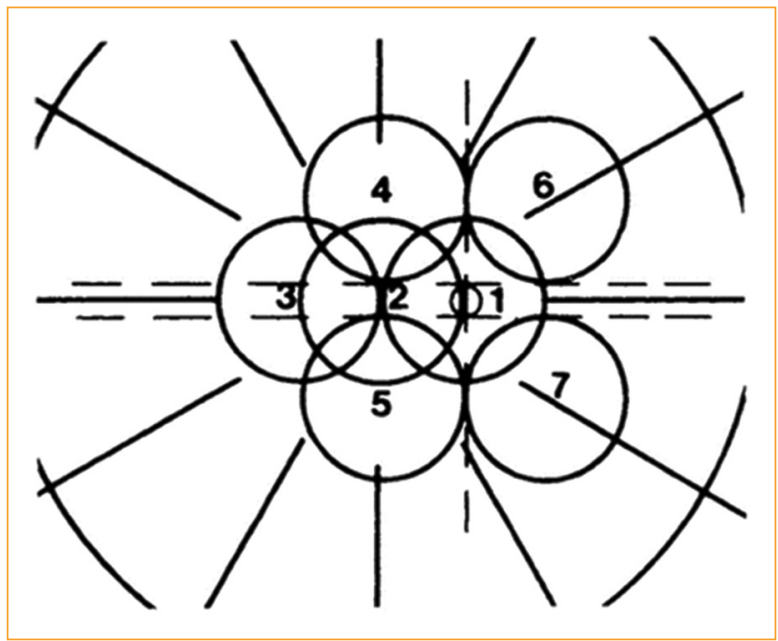

Figura 1. Los siete campos estandarizados de la clasificación modificada de Airlie House. Se ejemplifica en un ojo derecho. El campo 1 se centra en el nervio óptico, el 2 en la mácula y el 3 es temporal a la mácula.

\section{Clasificación internacional de la retinopatía diabética y del edema macular}

En el año 2003, el Global Diabetic Retinopathy Project Group propuso una clasificación en la que se determina una escala de gravedad5:

1. Ausencia aparente de edema macular diabético (EMD): sin engrosamiento o exudados duros aparentes en el polo posterior. 


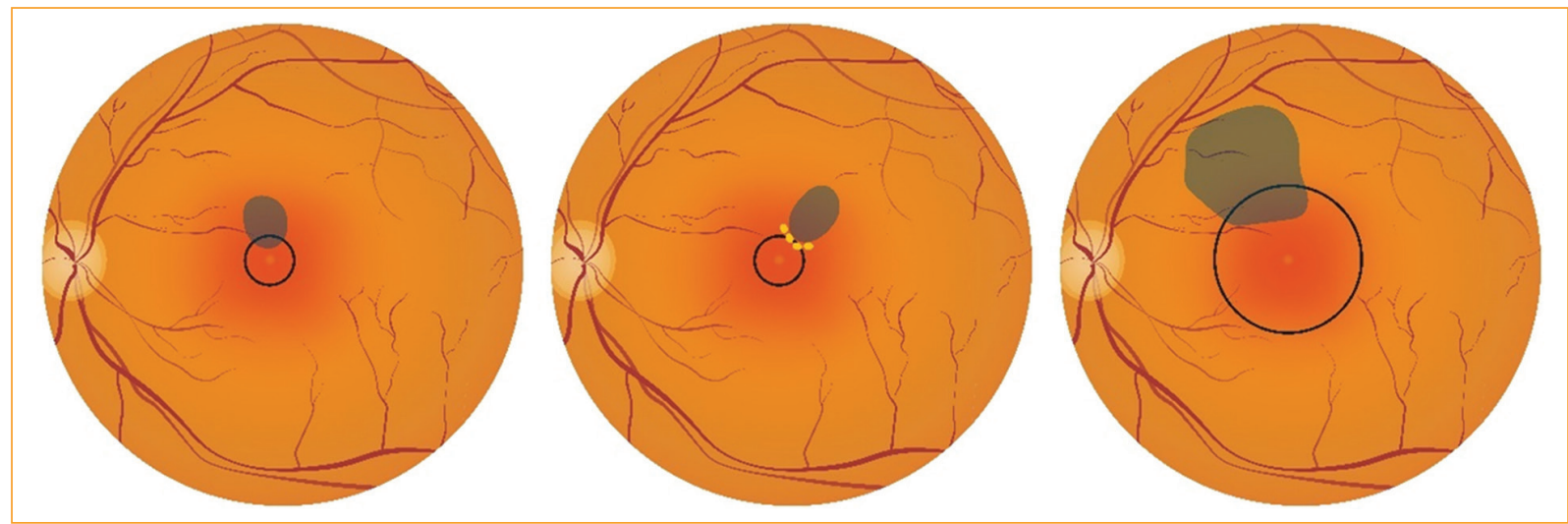

Figura 2. Esquema de la clasificación del edema macular diabético clínicamente significativo utilizada en el ETDRS.

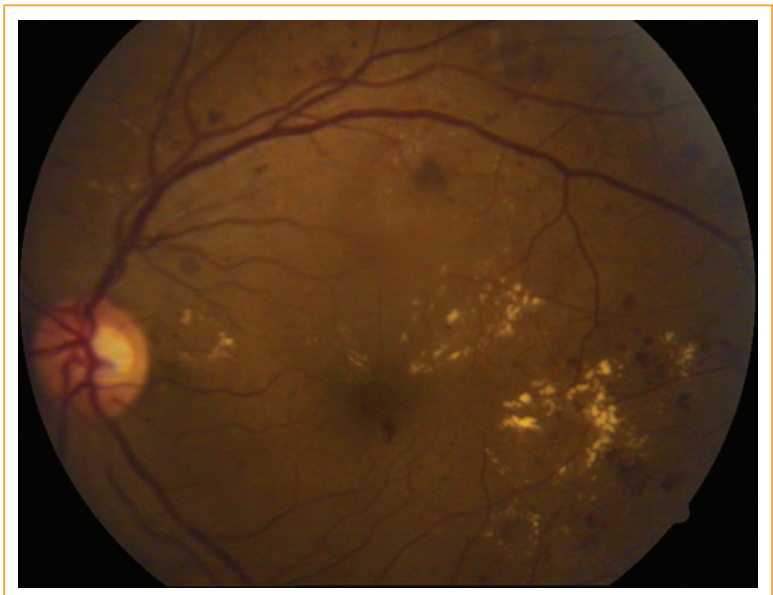

Figura 3. Ejemplo de un paciente con edema macular diabético clínicamente significativo con exudados duros circinados que respetan el centro de la fóvea.

2.EMD aparentemente presente: algún engrosamiento retiniano o exudados duros aparentes en el polo posterior. Puede categorizarse de la siguiente manera:

a) EMD leve: algún engrosamiento retiniano o exudados duros en el polo posterior, pero lejos del centro de la mácula.

b) EMD moderado: engrosamiento retiniano o exudados duros que alcanzan el centro de la mácula, pero no la afectan.

c) EMD grave: engrosamiento o exudados duros que afectan el centro de la mácula.

Esto requiere una evaluación tridimensional que se realiza mejor bajo midriasis con lámpara de hendidura o fotografía estereoscópica.

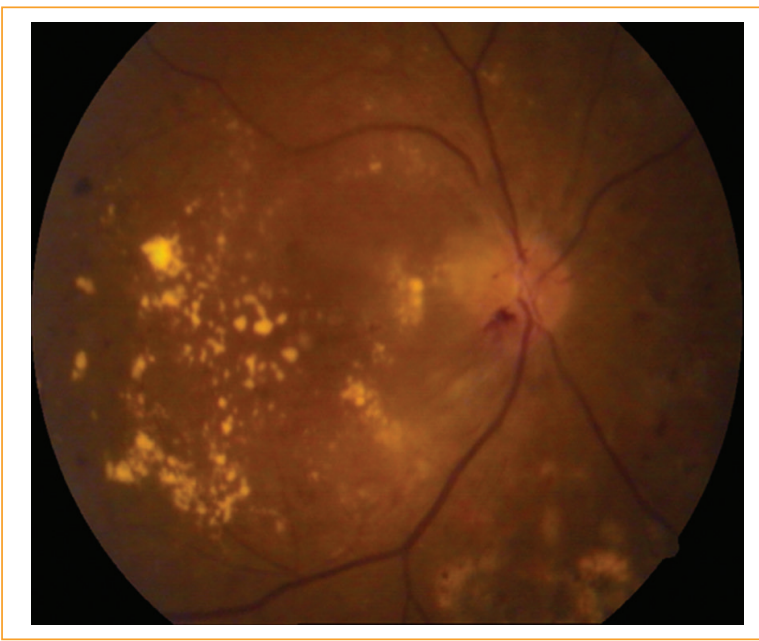

Figura 4. Ejemplo de un paciente con edema macular diabético y exudados duros que afectan el centro de la fóvea; además, tiene neovascularización en el disco óptico, vasos exangües, hemorragias y exudados por fuera de las arcadas.

\section{ETDRS frente a imágenes de campo ultraamplio}

Así mismo, la clasificación tradicional del ETDRS está siendo confrontada por las nuevas tecnologías que permiten adquirir imágenes de campo ultraamplio, lo que hace posible documentar cambios periféricos y detectar un subgrupo de pacientes que tienen un mayor riesgo de progresión de la retinopatía diabética que no se podría establecer con los siete campos tradicionales del ETDRS. Existe evidencia de que las áreas periféricas no perfundidas tendrían un papel en el desarrollo de EMD ${ }^{6-8}$. 


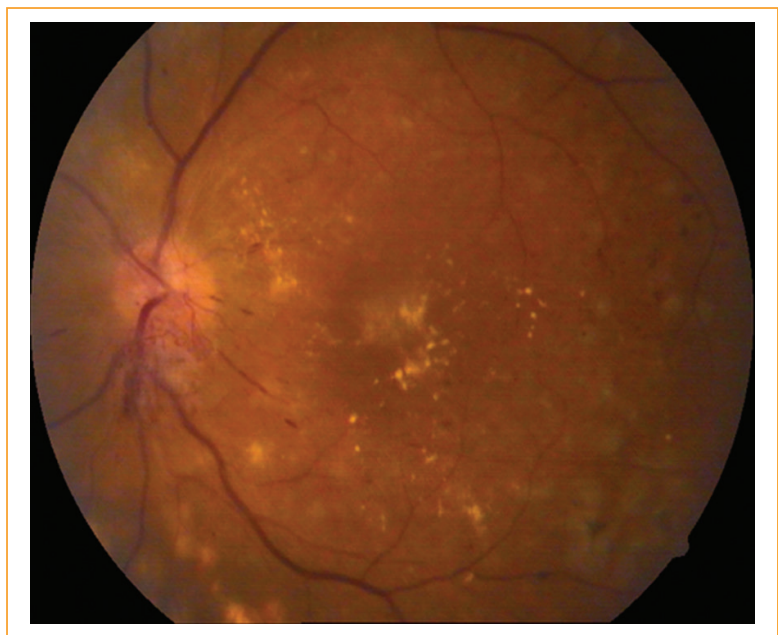

Figura 5. Ejemplo de un paciente con fotocoagulación panretiniana, neovasos en el disco de más de un tercio del diámetro papilar y exudados confluentes en la fóvea.

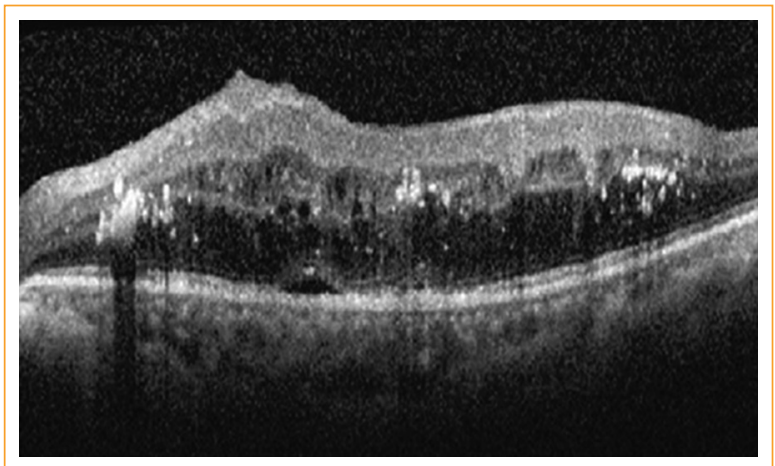

Figura 6. Ejemplo de engrosamiento difuso.

\section{Clasificación según la tomografía de coherencia óptica}

Con el advenimiento de la terapia con antiangiogénicos (anti-VEGF [vascular endothelial growth factor]) intravítreos, la evolución del edema macular y de la retinopatía diabética se ha visto modificada e incluso revertida, y con la aparición de la tomografía de coherencia óptica ha sido posible describir y reclasificar el EMD. Los avances tecnológicos en la tomografía de coherencia óptica han permitido incrementar la resolución y cuantificar el grosor de la retina y la coroides con mayor precisión, así como describir las diferentes capas de la retina ${ }^{9}$.

Se mencionan los siguientes tipos de edema macular ${ }^{9}$ :

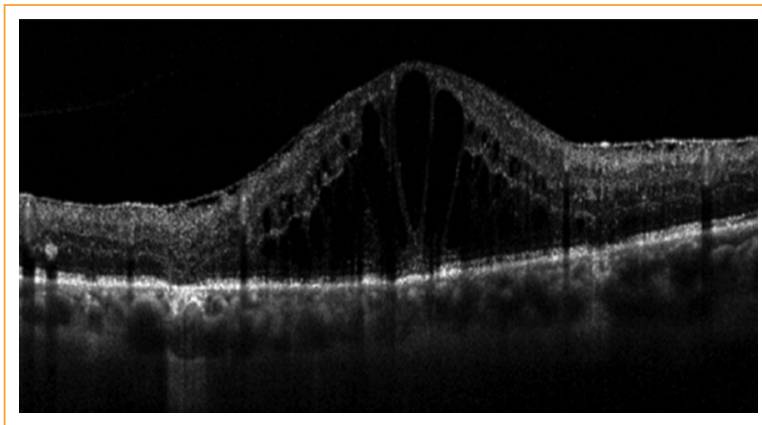

Figura 7. Edema macular cistoideo.

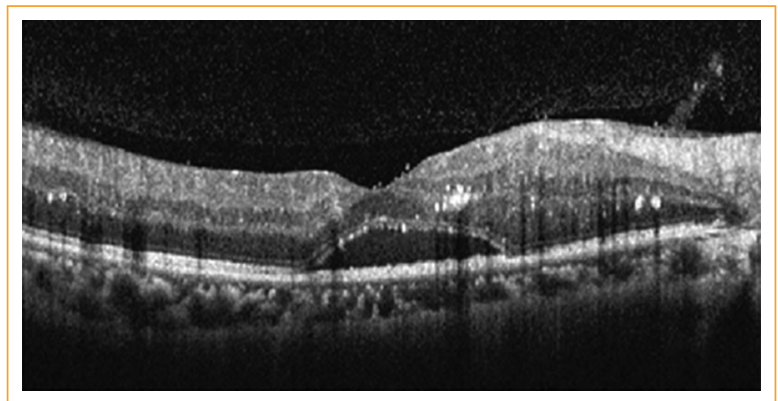

Figura 8. Desprendimiento seroso.
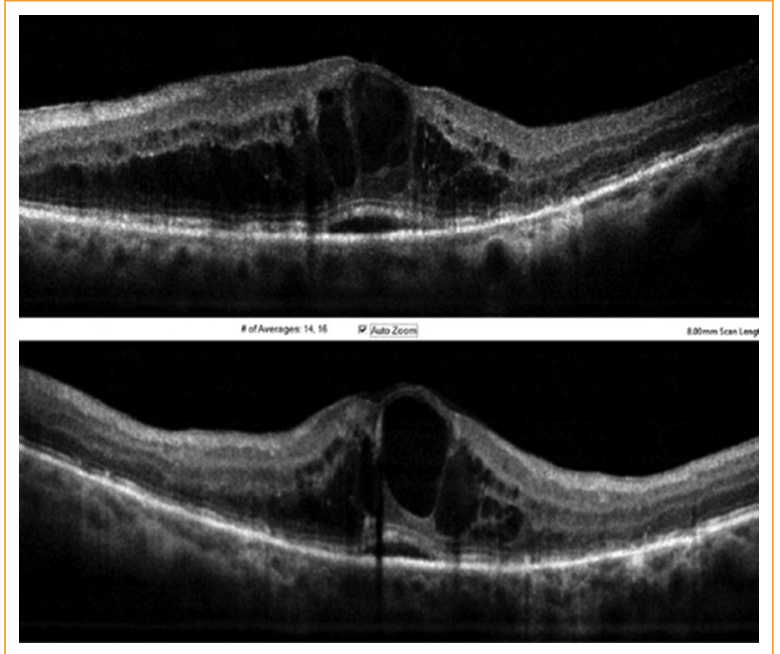

Figura 9. Ejemplo de edema macular de tipo mixto.

a) Engrosamiento difuso (tipo esponja) (figura 6).

b) Edema macular cistoideo (engrosamiento de la fóvea con cambios cistoideos) (figura 7).

c) Desprendimiento seroso de la retina (engrosamiento de la fóvea con líquido subretiniano) (figura 8).

d) Mixto (figura 9). 
Esta clasificación es importante porque hay evidencia que indica que los diferentes subtipos de edema tendrían una mejor o peor respuesta a alguna terapia en particular, ya sea inyección intravítrea de anti-VEGF o triamcinolona intravítrea o subtenoniana ${ }^{10}$.

\section{Bibliografía}

1. Goldberg M, Fine S. Symposium on the Treatment of Diabetic Retinopathy. Arlington: US Department of Health, Education and Welfare; 1968. Public Health Service Publication No. 1890.

2. The Diabetic Retinopathy Study Research Group. A modification of the Airlie House Classification of Diabetic Retinopathy. DRSreport \#7. Invest Ophthalmol Vis Sci. 1981;21:210-26

3. Early Treatment Diabetic Retinopathy Study Research Group. Grading diabetic retinopathy from stereoscopic color fundus photographs - an extension of the Modified Airlie House Classification. ETDRS Report Number 10. Ophthalmology. 1991;98:786-806.
4. Solomon S, Goldberg M. ETDRS grading of diabetic retinopathy: still the gold standard? Ophthalmic Res. 2019;62:190-5.

5. Wilkinson C, Ferris F, Klein R, Lee P, Agardh C, et al. Proposed international clinical diabetic retinopathy and diabetic macular edema disease severity scales. Ophthalmology. 2003;110:1677-82.

6. Schmidt U, García J, Bandello F, Berg K, Chakravarthy U, Gerendas BS, et al. Guidelines for the management of diabetic macular edema by the European Society of Retina Specialists (EURETINA). Ophthalmologica. 2017;237:185-222

7. Aiello L, Odia I, Glassman A, Melia M, Jampol L, Bressler N, et al.; Diabetic Retinopathy Clinical Research Network. Comparison of early treatment diabetic retinopathy study standard 7-field imaging with ultrawide-field imaging for determining severity of diabetic retinopathy. JAMA Ophthalmol. 2019;137:65-73.

8. Abramoff M, Fort P, Han I, Jayasundera T, Sohn E, Gardner T. Approach for a clinically useful comprehensive classification of vascular and neural aspects of diabetic retinal disease. IOVS. 2018;59:519-27.

9. Chung R, Kim Y, Ha S, Byeon H, Cho C, Kim J. Role of inflammation in classification of diabetic macular edema by optical coherence tomography. J Diabetes Res. 2019;2019:8164250.

10. Cho Y, Lee D, Kim M. Optical coherence tomography findings predictive of response to treatment in diabetic macular edema. J Int Med Res. 2018;46:4455-64. 


\title{
Edema macular diabético: diagnóstico clínico
}

\author{
Vanessa Flores-Peredo* \\ Hospital Regional Adolfo López Mateos, Instituto de Seguridad y Servicios Sociales de los Trabajadores del Estado, Ciudad de México, México
}

La principal causa de pérdida visual en los pacientes diabéticos es el edema macular diabético (EMD). La alteración que define la aparición del EMD es un engrosamiento de la retina en el área macular provocado por la extravasación de líquido al espacio extravascular. Requiere una evaluación tridimensional que se realiza mejor mediante un examen previa dilatación con biomicroscopía en lámpara de hendidura o estereofotografía del fondo de ojo'1.

La evaluación clínica del edema macular se caracteriza por su subjetividad. La oftalmoscopía directa e indirecta puede mostrar solo una alteración en el reflejo foveal. La fotografía estereoscópica del fondo y la biomicroscopía con lente de contacto desempeñan un papel importante en demostrar cambios en el volumen de la retina en el área macular, pero son dependientes de la experiencia del observador, y los resultados no ofrecen una medición reproducible del cambio de volumen. El engrosamiento de la retina puede acompañarse de exudados duros, que están formados por material lipídico y proteínico extravasado de los vasos de la retina y depositado en las capas externas de la retina, 0 incluso en ocasiones en el espacio subretiniano ${ }^{1,2}$.

Una exploración oftalmológica puede detectar clínicamente el EMD; los microaneurismas acompañados de exudados duros suelen ser los primeros signos visibles de EMD. Los microaneurismas se presentan como una herniación sacular de las paredes capilares y se aprecian como puntos rojos profundos que varían de 15 a $60 \mu \mathrm{m}$ de diámetro. El Early Treatment Diabetic Retinopathy Study (ETDRS) ha sido la base para la estandarización de la nomenclatura del EMD. Introduce

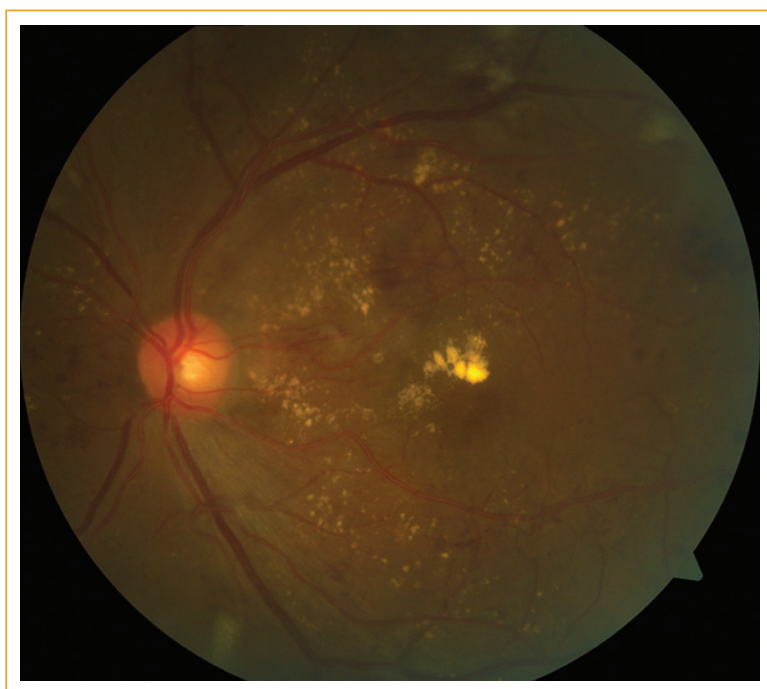

Figura 1. Edema macular diabético. Presencia de exudados duros.

los términos de EMD no clínicamente significativo y EMD clínicamente significativo. Se basa en la evaluación clínica del estado de la retina del polo posterior y en examinar con detalle la presencia de exudados duros y el espesor de la retina (figura 1$)^{3,4}$.

Se considera EMD clínicamente significativo aquel que cumple con alguna de las siguientes características:

- Engrosamiento retiniano a $500 \mu \mathrm{m}$ o menos del centro de la fóvea.

- Presencia de exudados duros dentro de las $500 \mu \mathrm{m}$ centrales si se asocian con engrosamiento retiniano adyacente. 
Tabla 1. Categorías de edema macular diabético de acuerdo con la guía ICO 2017

Sin EMD

EMD sin compromiso central

EMD con compromiso central

macur diabético.

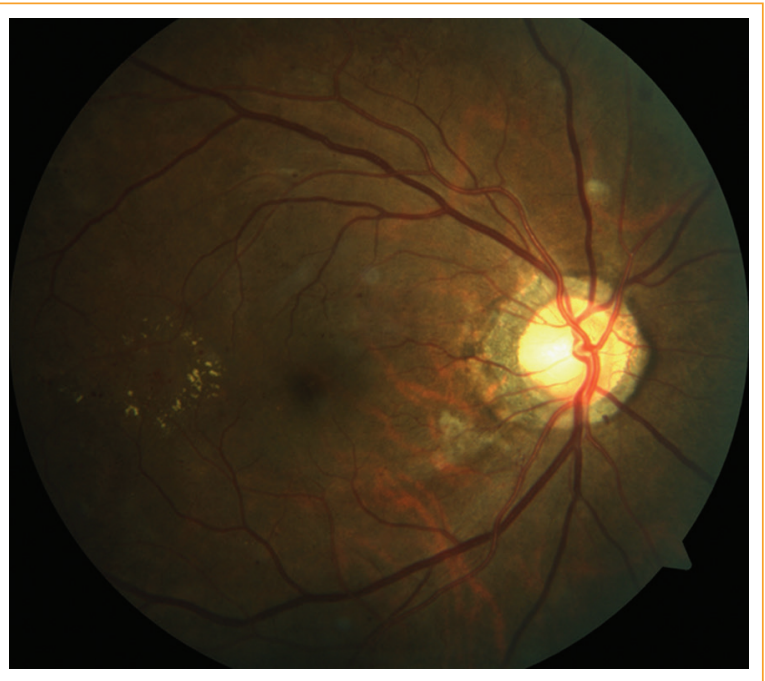

Figura 2. Edema macular sin compromiso central.

- Zonas de engrosamiento retiniano del tamaño de un diámetro papilar o mayor, y que parte del mismo se encuentra dentro de un disco de diámetro de la fóvea.

La manifestación clínica más relevante en el EMD es una disminución visual central, asociada a una deformación de las imágenes, que puede aparecer en estadios muy tempranos de la enfermedad. Su gravedad puede ser desde leve y asintomática hasta una pérdida de visión profunda ${ }^{1}$.

El EMD es una complicación adicional importante que se evalúa por separado de las etapas de la retinopatía, ya que se puede encontrar en los ojos con cualquier gravedad de retinopatía diabética y puede seguir un curso independiente. En la actualidad, según la guía publicada por el ICO 2017, los ojos diabéticos son clasificados como sin EMD, con EMD sin compromiso

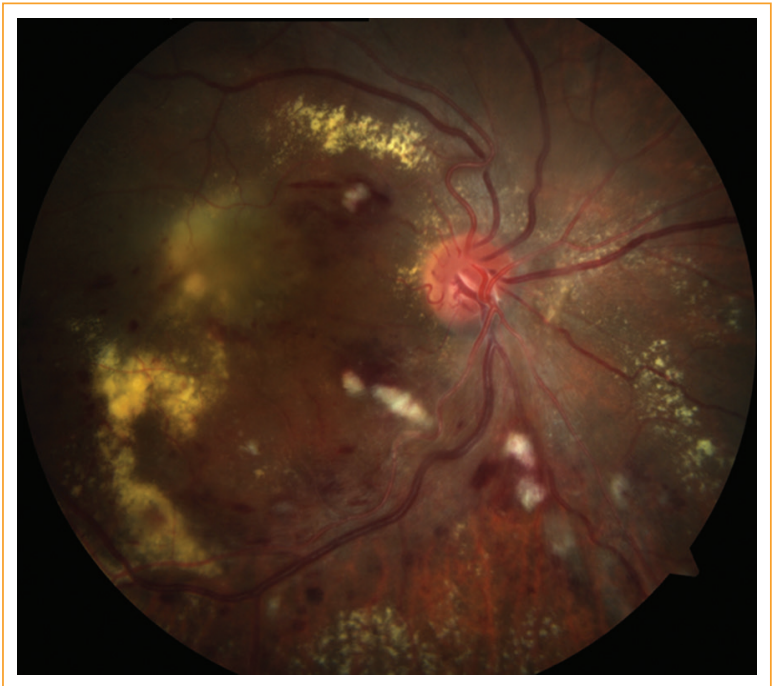

Figura 3. Edema macular con compromiso central.

central (figura 2) o con EMD con compromiso central (figura 3). La determinación de la gravedad del EMD según estas tres categorías establecerá la necesidad de tratamiento y las recomendaciones de seguimiento (tabla 1$)^{5}$.

\section{Bibliografía}

1. Andonegui J, Jiménez L. Edema macular diabético. An Sist Sanit Navar. 2008;31(Suppl 3):35-44.

2. Cunha J, Coscas G. Diagnosis of macular edema. Ophthalmologica. 2010;224(Suppl 1):2-7.

3. The Early Treatment Diabetic Retinopathy Study Research Group. Photocoagulation for diabetic macular edema. Early Treatment Diabetic Retinopathy Study report no 1. Arch Ophthalmol. 1985;103:1796-806.

4. Kinyoun J, Barton F, Fisher M, Hubbard L, Aiello L, Ferris F. Detection of diabetic macular edema. Ophthalmoscopy versus photography - Early Treatment Diabetic Retinopathy Study Report Number 5. Ophthalmology. 1989;96:746-50.

5. International Council of Ophthalmology. Updated 2017. ICO Guidelines for Diabetic Eye Care. San Francisco, EUA: International Council of Ophthalmology; 2017. 


\title{
Angiografía de retina con fluoresceína en el edema macular diabético
}

\author{
David Lozano-Rechy* \\ Instituto de Oftalmología FAP Conde de Valenciana, Ciudad de México, México
}

La evaluación de la circulación retiniana en el abordaje diagnóstico del edema macular diabético (EMD) tiene valor significativo, esencialmente debido a que demuestra la alteración de la barrera hematorretiniana, así como la ausencia de circulación que puede derivar en pérdida visual irreversible. Hoy en día, la angiografía

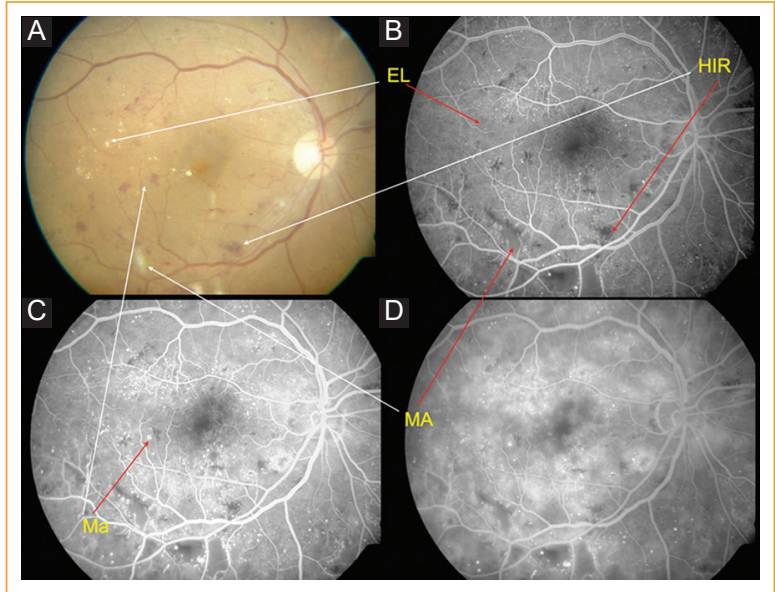

Figura 1. Fotografías de edema macular en un ojo derecho. A: fotografía en color que muestra hemorragias intrarretinianas, microaneurismas, exudados lipídicos y manchas algodonosas. B: angiografía de retina con fluoresceína (ARF) en fase arteriovenosa temprana. C: ARF en fase arteriovenosa media. D: ARF en fase de recirculación. Flecha blanca dirigida a fotografía de color y flecha roja a fotografía angiográfica señalan comparativamente hemorragia intrarretiniana (HIR), microaneurisma (Ma), exudados lipídicos (EL) y manchas algodonosas (MA) en una zona de engrosamiento retiniano representativo de edema macular. de retina con fluoresceína (ARF) es el método económicamente más accesible para esta evaluación y permite identificar, cuantificar y documentar las alteraciones vasculares, al inyectar este colorante por vía intravenosa, que mostrará la circulación contrastando en la retina lo que tiene y no tiene fluoresceína ${ }^{1-3}$.

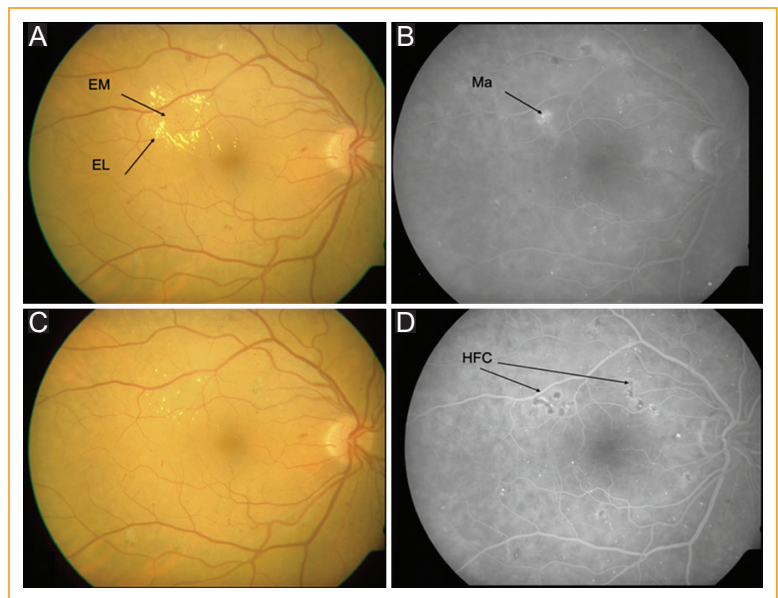

Figura 2. Fotografías de edema macular en un ojo derecho. A: fotografía en color que muestra exudación lipídica (EL) en patrón circinado, rodeando a una zona de edema macular (EM) que no involucra a la fóvea. B: angiografía de retina con fluoresceína (ARF) que muestra microaneurismas (Ma) con hiperfluorescencia por filtración. C: fotografía en color que muestra la desaparición casi completa de la exudación lipídica y del edema macular posterior a fotocoagulación focal con láser. D: ARF que muestra zonas de hipofluorescencia por huellas de fotocoagulación (HFC) con láser. 


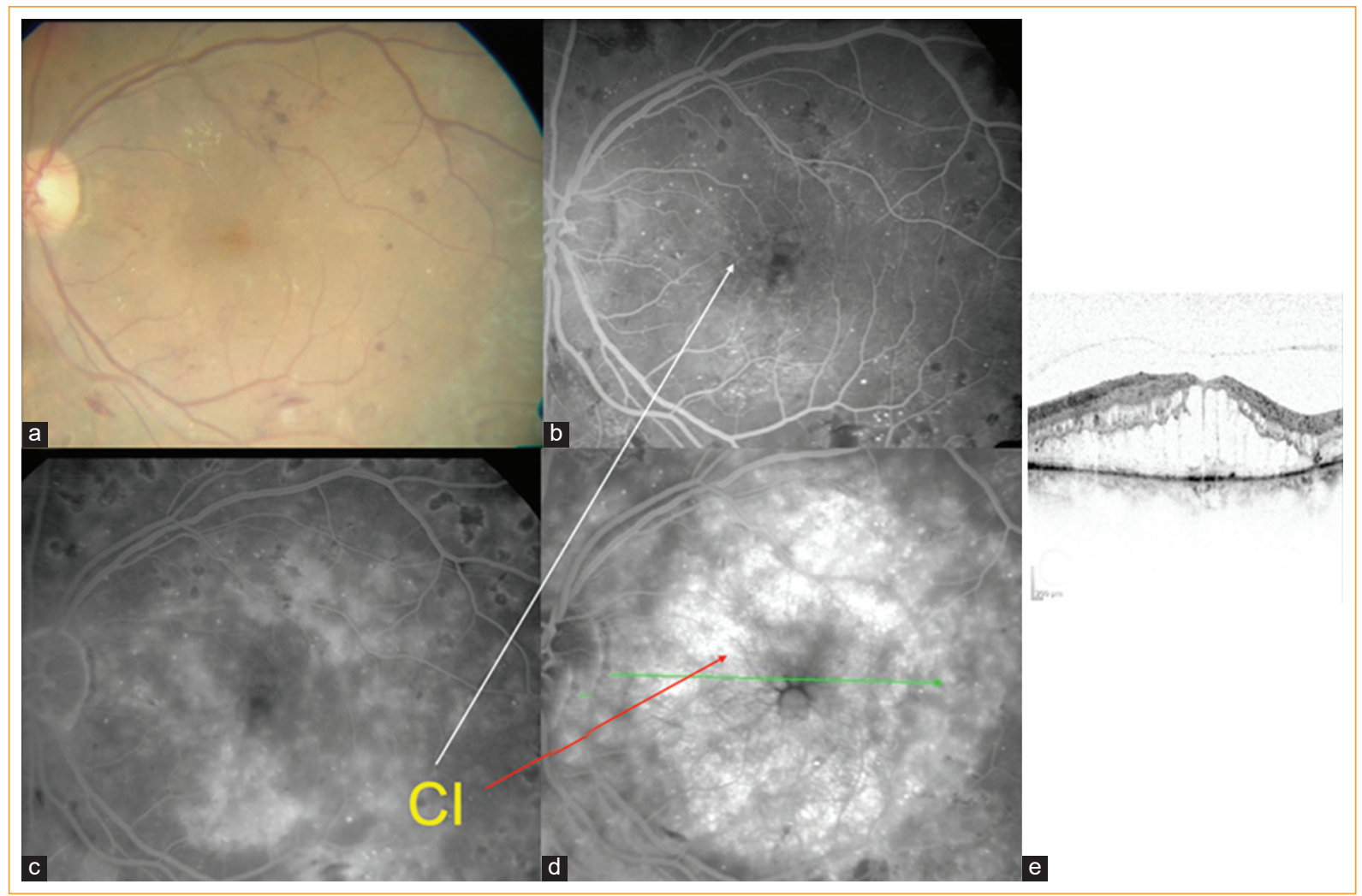

Figura 3. Fotografías de edema macular en un ojo izquierdo. A: fotografía en color que muestra muy escasa exudación lipídica y hemorragias intrarretinianas. B: angiografía de retina con fluoresceína (ARF) en fase arteriovenosa media que muestra muy escasos microaneurismas. C: ARF en fase arteriovenosa tardía que muestra el inicio de la visualización de la filtración. D: ARF en fase de recirculación que muestra filtración difusa de capilares. E: corte de tomografía en la región central foveal. Flecha blanca dirigida a ARF de fase arteriovenosa media en región que no muestra microaneurismas y flecha roja dirigida a ARF en fase de recirculación (tardía) en la misma región macular, pero que muestra filtración de capilares incompetentes (CI). Línea verde indica región de corte tomográfico macular.

Las alteraciones biomicroscópicas que se identifican en la retinopatía diabética con edema macular pueden ser documentadas como hiperfluorescentes o hipofluorescentes.

Las que producen hiperfluorescencia son representativas de un comportamiento anormal de la circulación retiniana, tanto en morfología como en función. Los microaneurismas, que representan saculaciones de capilares retinianos por pérdida de pericitos a consecuencia del daño hiperglucémico, suelen ser muy difíciles de identificar con la biomicroscopía, pero la ARF permite visualizarlos con mucha facilidad como puntos hiperfluorescentes que aumentan en intensidad y extensión, fenómeno que se conoce como hiperfluorescencia por filtración; este hallazgo angiográfico se observa en

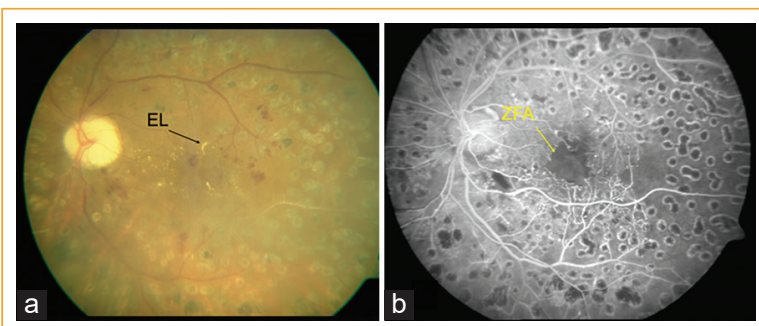

Figura 4. fotografías de edema macular en un ojo izquierdo. A: fotografía en color que muestra exudación lipídica (EL) alrededor de la fóvea, que a su vez presenta un engrosamiento representativo de edema macular. B: angiografía de retina con fluoresceína que muestra microaneurismas, aunque con hipofluorescencia por falta de llenado de la red vascular perifoveal, representativo de isquemia macular y causa irreversible de baja visual. 
el EMD focal o multifocal (figura 1). La visualización de microaneurismas es de gran valor para el oftalmólogo que decide llevar a cabo un tratamiento de fotocoagulación como manejo del EMD (figura 2) ) $^{4-6}$.

Existe también un fenómeno de filtración de fluoresceína (hiperfluorescencia por filtración) en el EMD que no proviene de microaneurismas, sino de capilares incompetentes, fenómeno que desarrolla engrosamiento macular extenso por filtración generalizada o difusa de suero sanguíneo a través de los capilares perifoveales, que además desarrollan espacios cistoides. Este tipo de edema se ha catalogado como edema macular difuso (figura 3) y suele tener un efecto favorable de resolución al promover el control de la nefropatía diabética e instaurar terapia antiangiogénica ${ }^{7}$.

Los fenómenos de hipofluorescencia están asociados a cuatro alteraciones de la retinopatía diabética:

- Hemorragias que suelen producir bloqueo de la fluorescencia coroidea, ya que se encuentran en el espesor de la retina.

- Exudados lipídicos, también localizados en el espesor de la retina.

- Manchas algodonosas, representativas de microinfartos de la capa de fibras nerviosas de la retina, que además reflejan ausencia de perfusión capilar a ese nivel (figura 1).
- Falta de llenado de la red vascular perifoveal (figura 4), fenómeno llamado isquemia macular, el más importante por su significancia clínica, que suele desarrollar deterioro visual irreversible. Identificar la isquemia macular es útil para informar al paciente del pobre pronóstico de recuperación visual al estar bajo tratamiento del $\mathrm{EMD}^{7,8}$.

\section{Bibliografía}

1. Salz D, de Carlo T, Adhi M, Moult E, Choi W, Baumal C, et al. Select features of diabetic retinopathy on swept-source optical coherence tomographic angiography compared with fluorescein angiography and normal eyes. JAMA Ophthalmol. 2016;134:644-50.

2. Gass J, Sever R, Sparks D. A combined technique of fluorescein funduscopy and angiography of the eye. Arch Ophthalmol. 1967;78:455-61.

3. Ayyakkannu M, Plskova J, Farrow A, McKay S, Sharp P, Forrester J. Ultra-wide-field fluorescein angiography of the ocular fundus. Am J Ophthalmol. 2005;140:525-7.

4. Bresnick G. Diabetic macular edema: a review. Ophthalmology. 1986;93:989-97.

5. Lee S, Chhablani J, Chan C, Wang H, Barteselli G, El-Emam S, et al. Characterization of microaneurysm closure after focal laser photocoagulation in diabetic macular edema. Am J Ophthalmol. 2013;155: 905-12.

6. Kozak I, Oster S, Cortes M, Dowell D, Hartmann K, Kim J, et al. Clinical evaluation and treatment accuracy in diabetic macular edema using navigated laser photocoagulator - NAVILAS. Ophthalmology. 2011;118:1119-24.

7. Liu Z, Ma X, Liao D, Liu X, Bai L, Yao J, et al. Association of urinary albumin excretion with central foveal thickness and intravitreal conbercept treatment frequency in patients with diabetic macular edema. Int J Ophthalmol. 2019;12:1598-604.

8. Dawn A, Pearse K, Zarranz J, Fung S, Powner M, Platteau E, et al. The effects of macular ischemia on visual acuity in diabetic retinopathy. Invest Ophthalmol Vis Sci. 2013:54:2353-60. 


\title{
Tomografía de coherencia óptica en el edema macular diabético
}

\author{
Renata García-Franco* \\ Instituto de la Retina del Bajío INDEREB, Santiago de Querétaro, Querétaro, México
}

La tomografía de coherencia óptica (OCT) es un estudio de imagen no invasivo que permite observar imágenes transversales y micrométricas de la retina in vivo. Mide la reflectividad óptica de los tejidos retinianos utilizando la luz como fuente de emisión. El funcionamiento de la OCT es similar al del ultrasonido, con la diferencia de que la OCT utiliza una fuente de luz cercana al espectro infrarrojo $(820-830 \mathrm{~nm})$ en lugar de ondas acústicas, basándose en el principio de interferometría. Este principio funciona agregando o interfiriendo ondas de dos rayos de luz para calcular el tiempo de retraso y la magnitud de luz reflejada y esparcida en los tejidos, comparándola con un haz de luz de referencia. La luz coherente reflejada proporciona información sobre el retraso de esta en las distintas capas de la retina, lo que permite conocer la localización longitudinal de las diferentes zonas de reflexión, la distancia y el espesor de las diversas estructuras oculares (cortes axiales o A-scan). El sistema proporciona múltiples cortes axiales (A-scan) contiguos que permiten construir con gran exactitud un mapa dimensional de las zonas de reflexión, que finalmente se observa como una imagen topográfica bidimensional (B-scan). Esto permite evaluar cuantitativamente (grosor retiniano) y cualitativamente (morfología de las capas de la retina neurosensorial e interfase vitreorretiniana) la retina (figura 1). Los nuevos equipos que poseen tecnología de dominio espectral (SD) obtienen imágenes tomográficas con resoluciones de 5-10 $\mu \mathrm{m}^{1}$.
Esta herramienta es la más importante para la evaluación, el diagnóstico y el seguimiento de la respuesta al tratamiento del edema macular diabético (EMD), permitiendo de forma objetiva, eficaz y reproducible:

- Cuantificar el grosor macular.

- Cuantificar el volumen macular.

- Identificar las capas de la retina y su disrupción.

- Valorar la interfase vitreorretiniana.

- Evaluar la respuesta al tratamiento.

\section{Clasificación del edema macular diabético por tomografía de coherencia óptica}

Panozzo, et al. ${ }^{2}$ clasificaron el EMD de acuerdo con su morfología y la presencia de tracción vitreorretiniana. Según su morfología puede ser:

- E1: engrosamiento simple (espongiforme).

- E2: engrosamiento cistoideo.

- E3: desprendimiento del neuroepitelio.

Según la presencia de tracción retiniana puede ser:

- T0: ausencia de tracción vitreomacular.

- T1: adhesión vitreomacular. Línea hiperreflectiva adherida uniformemente a la superficie de la retina sin distorsionarla.

- T2: tracción vitreomacular. Línea hiperreflectiva con varios puntos de adhesión en la retina que genera distorsión de esta.

- T3: tracción anteroposterior con configuración en «alas de gaviota». 


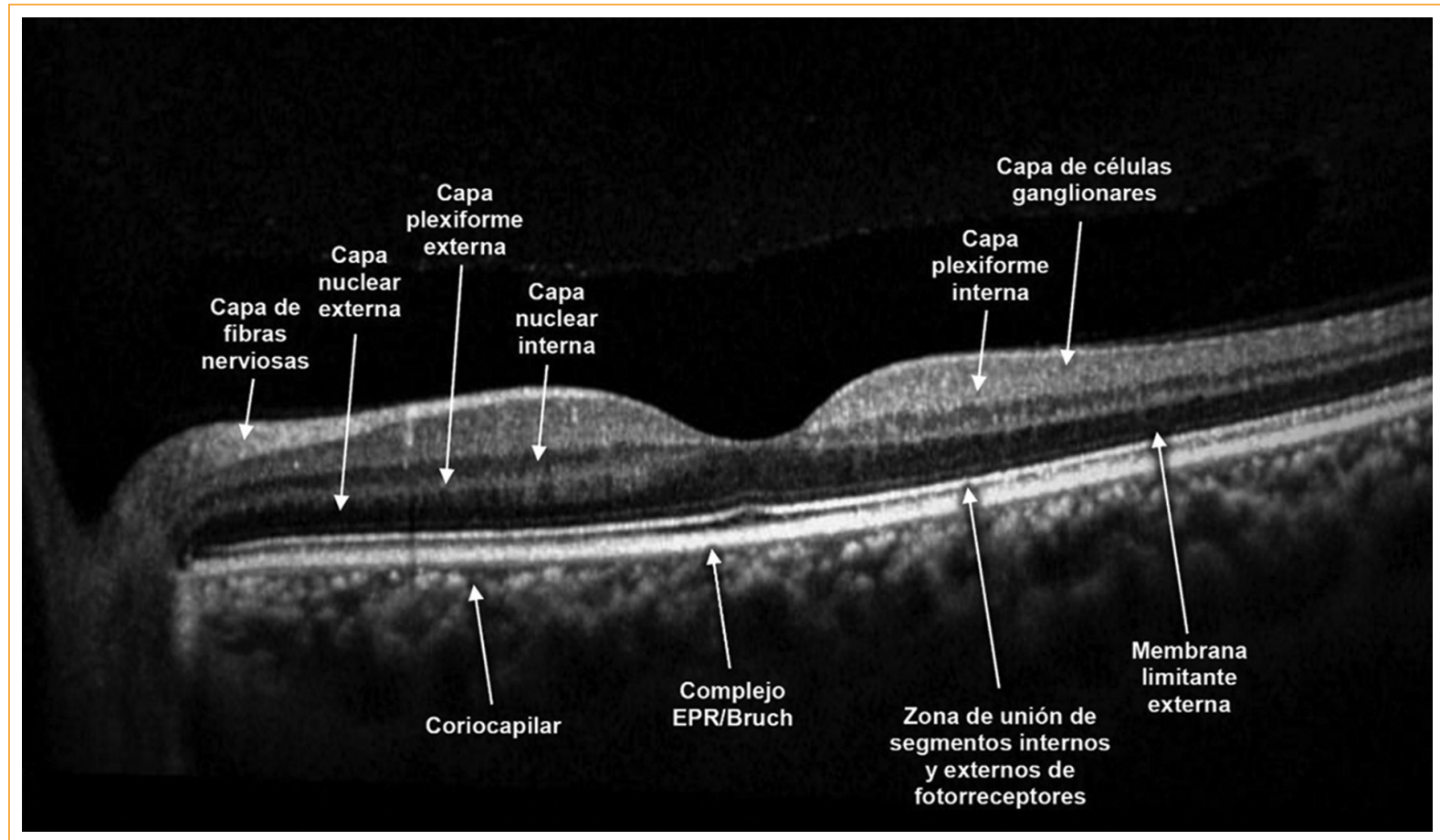

Figura 1. OCT macular de un sujeto sano con sus respectivas capas retinianas.

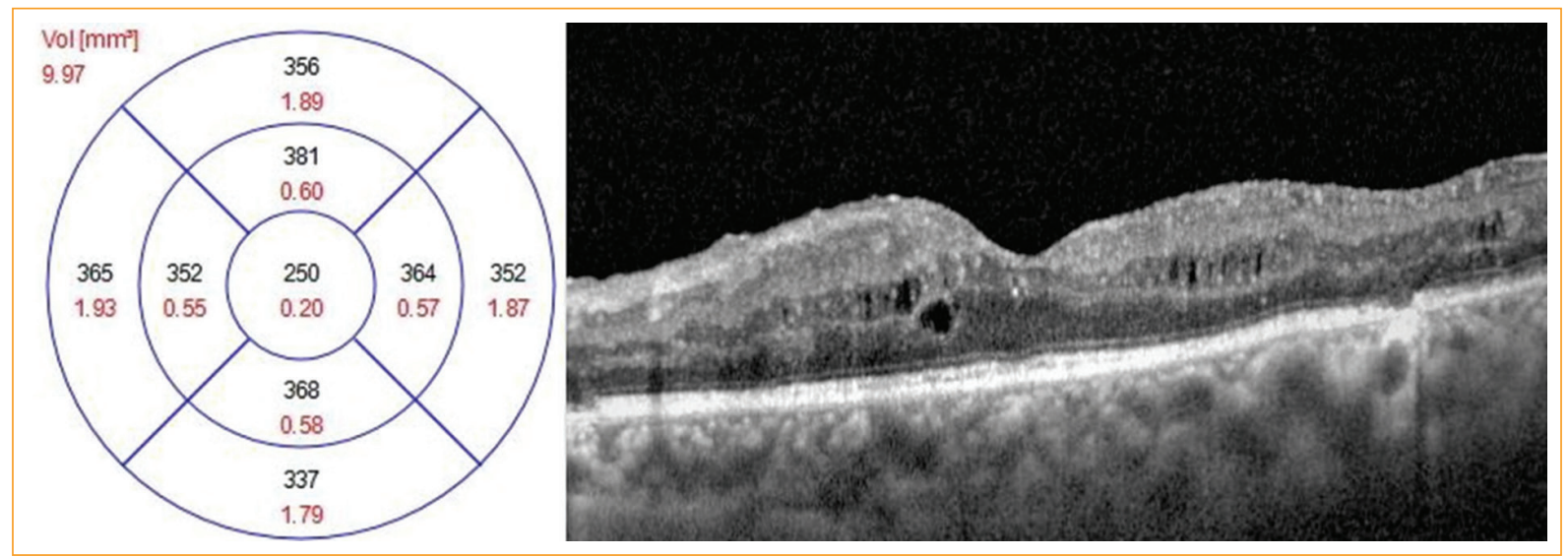

Figura 2. OCT macular de un paciente con edema macular diabético espongiforme (predomina el engrosamiento retiniano difuso).

\section{Edema macular diabético espongiforme}

También llamado engrosamiento retiniano difuso, es el más frecuente de todos y se debe a una alteración de las capas nuclear interna y plexiforme externa por acumulación de líquido en el espacio extracelular, que se expresa como un engrosamiento tisular asociado a una disminución difusa de la reflectividad total de la retina (figura 2).

\section{Edema macular diabético cistoideo}

Este tipo de edema está asociado a un mayor volumen macular y peor visión, y se caracteriza por unos espacios redondos de baja reflectividad separados por membranas delgadas de reflectividad normal que corresponderían a la elongación de las células de Müller y bipolares. Las lesiones cistoideas usualmente predominan en las capas internas de la retina y se ha 


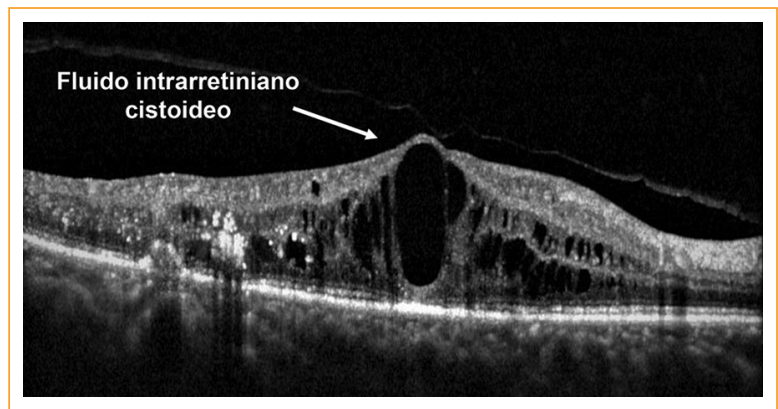

Figura 3. OCT macular de paciente con edema macular diabético cistoideo (nótese el fluido intrarretiniano cistoideo como imágenes hiporreflectivas circulares que predominan en la retina interna).
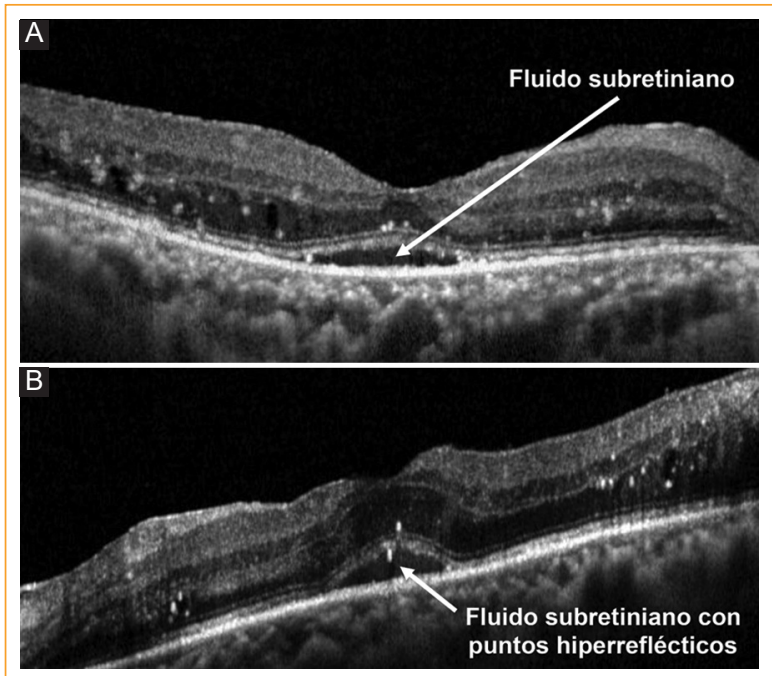

Figura 4. OCT macular de un paciente con edema macular diabético y desprendimiento seroso de la retina neurosensorial (nótese el fluido subretiniano como una imagen hiporreflectiva por encima del epitelio pigmentado de la retina) (A). Se señalan también algunos puntos hiperreflectivos en el espacio subretiniano (B).

intentado clasificarlas según su tamaño: pequeñas $(<100 \mu \mathrm{m})$, grandes $(101-200 \mu \mathrm{m})$ o gigantes $(>200 \mu \mathrm{m})$; las más grandes son las de peor visión basal y peor pronóstico visual (figura 3) $)^{3,4}$.

\section{Edema macular diabético asociado a desprendimiento seroso del neuroepitelio}

Este subtipo de edema puede presentarse aislado o en asociación con las lesiones cistoideas, y estaría causado por una alteración de la permeabilidad

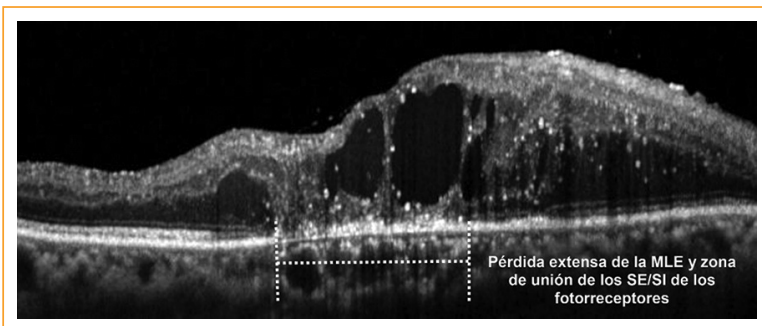

Figura 5. OCT macular de un paciente con edema macular diabético que muestra la extensión de la pérdida de integridad de la membrana limitante externa (MLE) y la zona de unión de los segmentos externo/ interno (SE/SI) de los fotorreceptores (líneas blancas discontinuas).

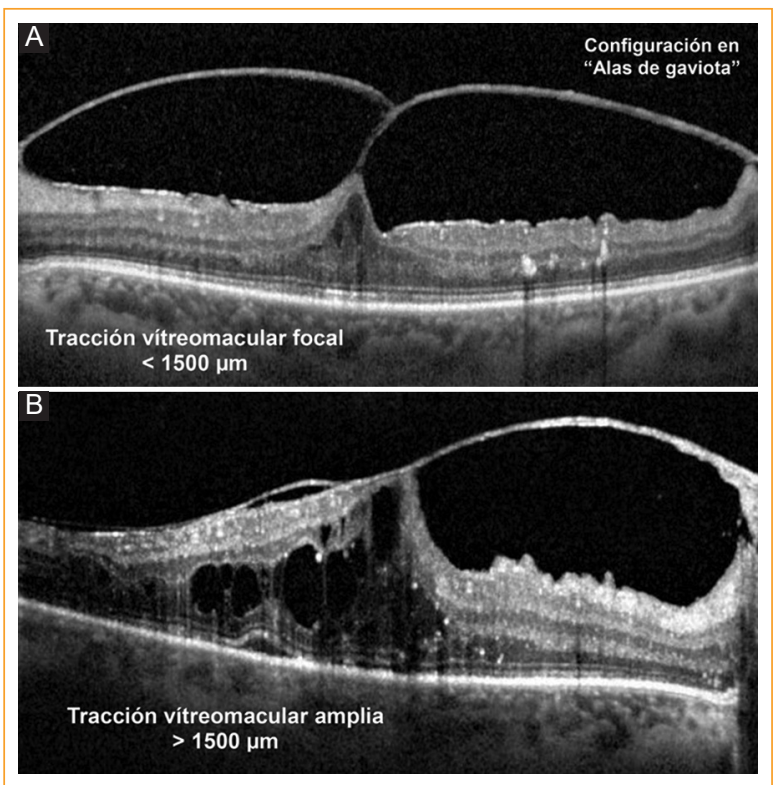

Figura 6. OCT macular que muestra la imagen de edema macular diabético con tracción vitreomacular tanto focal (A) como amplia (B). Nótese la configuración en «alas de gaviota" (A) debido a la tracción anteroposterior en el área foveal.

vascular de la perifóvea. Es el que mejor responde al tratamiento con antiangiogénicos intravítreos. Se ha encontrado una asociación de este tipo de edema con la hipertensión arterial sistémica. También se han reportado valores de interleucina 6 y de molécula de adhesión intercelular 1 elevados en el humor vítreo y el humor acuoso. Algunas veces se pueden observar puntos hiperreflectivos en el espacio subretiniano que estarían relacionados con un peor pronóstico visual y descontrol metabólico (figura 4 A y B $)^{5-7}$. 


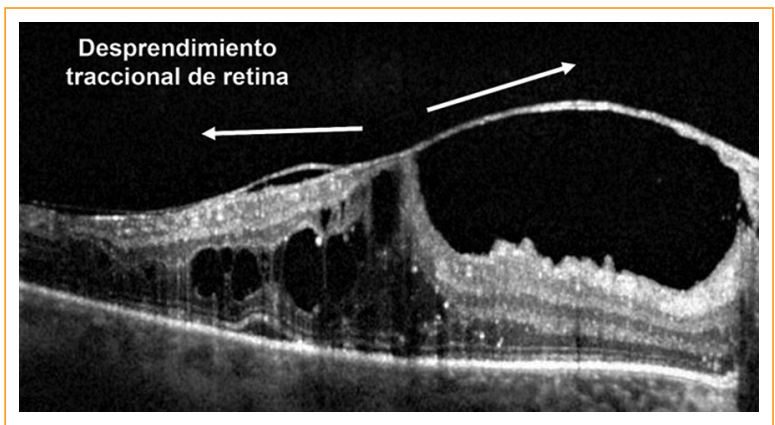

Figura 7. OCT macular que muestra un edema macular diabético con desprendimiento traccional de la retina. Nótese la tracción anteroposterior y tangencial (flechas blancas).

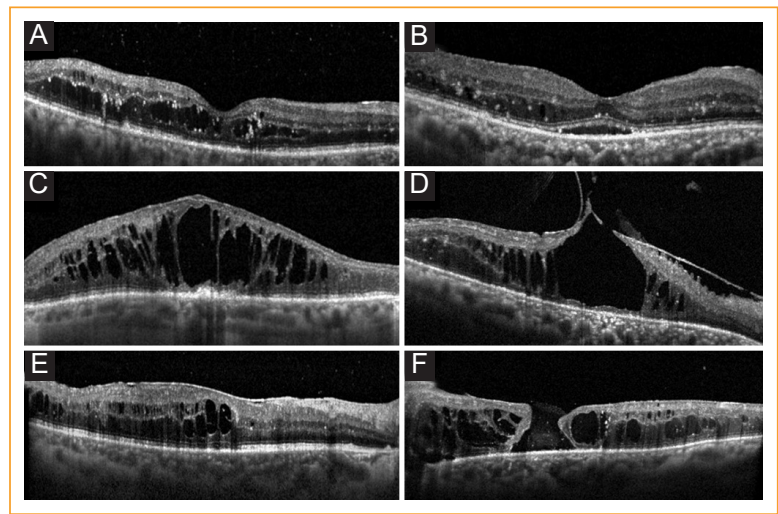

Figura 8. Imágenes de OCT macular que muestra un edema macular diabético (EMD) asociado a distintos hallazgos. A: EMD asociado a exudados duros, lesiones cistoideas y puntos hiperreflécticos. B: EMD asociado a fluido subretiniano y múltiples puntos hiperreflectivos. C: EMD con grandes cavidades cistoideas, además de pérdida de la membrana limitante externa (MLE) y zona de unión de los segmentos externos/internos (SE/SI) de los fotorreceptores. D: grandes cavidades cistoideas con pérdida extensa de la MLE y zona de unión de SE/SI de los fotorreceptores y tracción anteroposterior con formación de un agujero macular lamelar. E: EMD cistoideo asociado a membrana epirretiniana (nótese la línea hiperreflectiva adherida a toda la superficie interna de la retina). F: EMD cistoideo asociado a agujero macular de espesor completo.

La integridad de la membrana limitante externa (MLE), así como la de la zona elipsoide (segmentos internos de los fotorreceptores), y la presencia de exudados duros subfoveales, están directamente relacionadas con el pronóstico visual después del tratamiento tanto médico como quirúrgico (figura 5$)^{8}$.

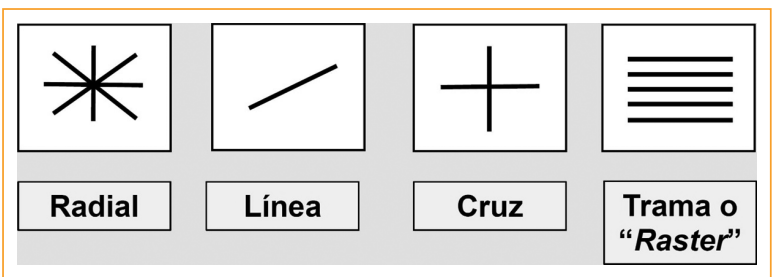

Figura 9. Imágenes de los distintos tipos de protocolos para el análisis macular.

\section{Edema macular diabético con tracción en la interfase vitreorretiniana}

Cuando la hialoides aumenta en grosor y se desprende parcialmente de la retina puede ejercer tracción tanto tangencial como anteroposterior sobre la superficie retiniana, generando edema macular o exacerbándolo si ya estuviese presente. La clasificación del International Vitreomacular Traction Study Group considera la extensión horizontal de la adhesión o tracción para su clasificación (figura 6), pudiendo ser focal $(<1500 \mu \mathrm{m})$ o amplia (> $1500 \mu \mathrm{m})$. Otro patrón morfológico de tracción anteroposterior es la configuración en "alas de gaviota» (figura $6 \mathrm{~A}$ ), que se observa cuando el desprendimiento del vítreo posterior esta adherido al centro foveal. En los pacientes con edema macular crónico y grandes cavidades cistoideas se ha descrito un patrón "similar al de un volcán», que es visto por OCT como una imagen hiperreflectiva en el vítreo que característicamente se ve emergiendo de la interfase vitreomacular (figura 6 B). Se cree que se produce por una salida progresiva de fluido del espacio intrarretiniano a la cavidad vítrea9,10.

El tratamiento quirúrgico está indicado cuando el EMD tiene componente traccional (figura 7). Este tipo de edema se ha asociado a un mal pronóstico visual después del tratamiento tanto farmacológico (antiangiogénicos o implante de corticoide de liberación prolongada) como quirúrgico (vitrectomía posterior con remoción de la hialoides engrosada y la membrana limitante interna), y estaría causado por el daño en las capas internas y externas de la retina generado por la tracción crónica.

La SD-OCT también ha sido valiosa para identificar otros hallazgos (figura 8) asociados al EMD:

- Hemorragia subretiniana, intrarretiniana 0 premacular.

- Membrana epirretiniana.

- Exudados blandos o duros.

- Puntos hiperreflectivos. 


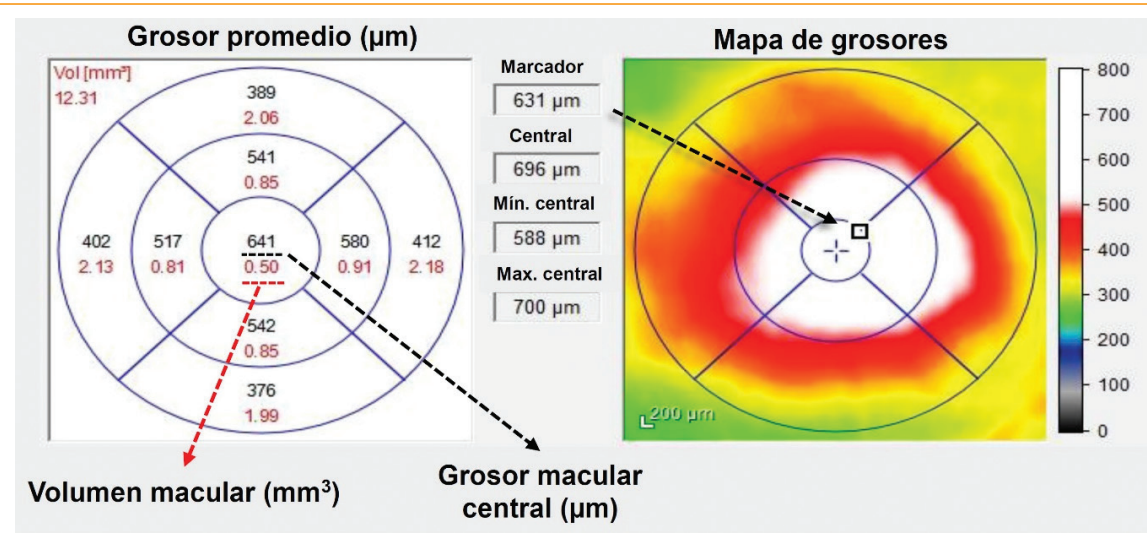

Figura 10. Protocolos de OCT macular para la evaluación del edema macular diabético. Nótese el grosor macular en las distintas áreas de la mácula (imagen de la izquierda), además del análisis volumétrico tanto total como en los diferentes sectores (flecha roja discontinua). En la imagen derecha se observa el mapa de grosores que muestra con colores calientes (rojo) las áreas de engrosamiento retiniano. Además, se pueden ver valores en la columna central que reflejan el grosor central promedio, máximo y mínimo, además de un área que puede señalarse con un marcador (flecha negra discontinua).

- Disrupción de la MLE y zona de unión de los segmentos internos y externos de los fotorreceptores.

- Agujero macular lamelar o de espesor completo.

- Fibrosis subretiniana.

- Desprendimiento seroso de retina neurosensorial.

- Atrofia macular.

\section{Tomografía de coherencia óptica: protocolos para seguimiento}

La SD-OCT proporciona no solo información cualitativa sobre las características de las capas retinianas, sino también medidas objetivas (análisis cuantitativo) que permiten la medición microscópica de varias estructuras que finalmente ayudarán a hacer una evaluación adecuada durante el seguimiento y el tratamiento de esta enfermedad.

\section{Análisis radial, en línea, en cruz y de líneas en trama}

- Radial: este protocolo se compone de una serie de 6 a 24 barridos lineales equidistantes $\left(30^{\circ}\right)$ desde un eje central común (centro macular), como los radios de una rueda de bicicleta. El patrón contiene seis líneas de $6 \mathrm{~mm}$ de longitud y es posible ajustar la longitud de todas las líneas del barrido (figura 9).

- Línea: realiza varios barridos lineales de $5 \mathrm{~mm}$ que se pueden repetir 0 adaptar individualmente (figura 9).
- Cruz (cross hair): se compone de dos barridos lineales $(3 \mathrm{~mm})$ perpendiculares que se cruzan en sus centros formando una cruz. Este protocolo resulta útil para examinar determinadas zonas de interés clínico en cortes que atraviesan los centros de los cuatro cuadrantes (figura 9).

- Líneas en trama (raster lines): se compone de una serie de 6 a 24 barridos lineales, paralelos y equidistantes, efectuados sobre una región rectangular de un tamaño especificado por el usuario, y permite examinar rigurosamente una región de cierto tamaño en la retina (figura 9).

\section{Análisis del grosor macular central}

El grosor macular central o grosor del subcampo central (cuantificado en $\mu \mathrm{m}$ ) es el parámetro más evaluado y usado en la mayoría de los ensayos clínicos y protocolos (figura 10). Los límites de esta medida van desde la superficie de la retina interna al borde interno del epitelio pigmentado de la retina, el cual se observa como una banda hiperreflectiva. La medida del grosor macular central es la más objetiva para evaluar la respuesta al tratamiento, ya sea con láser, antiangiogénicos, esteroides intravítreos o cirugía. Este parámetro, junto con la respuesta visual, son los más usados para establecer criterios de éxito, falla o retratamiento en la mayor parte de los ensayos clínicos. Se han considerado valores normales de $270.2 \pm 22.5 \mu \mathrm{m}$ para el OCT SPECTRALIS. 


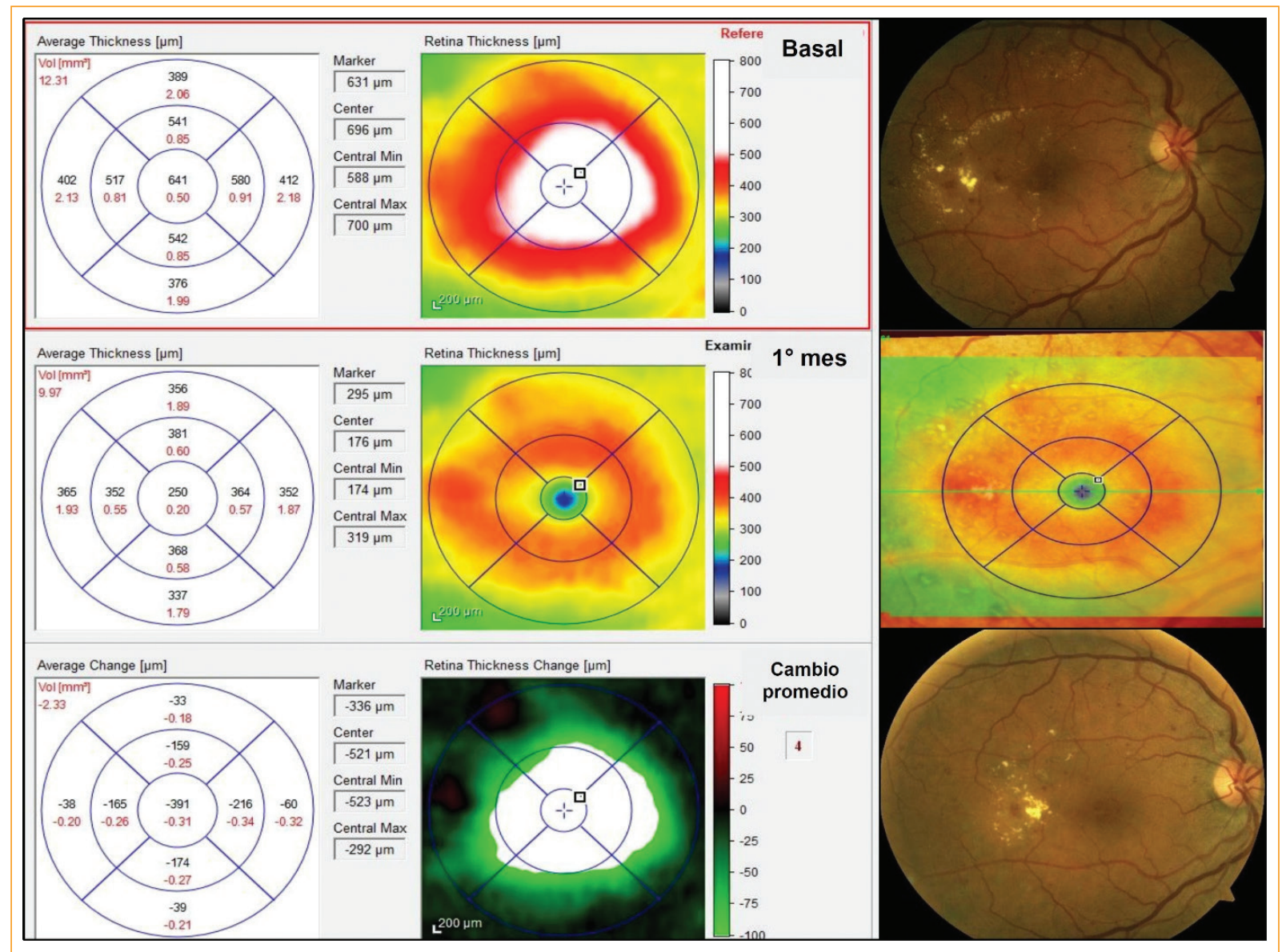

Figura 11. Protocolo de mapa macular rápido para la evaluación de seguimiento del edema macular diabético posterior al tratamiento antiangiogénico. Nótese la imagen inferior que muestra el cambio promedio del grosor con respecto al valor basal.

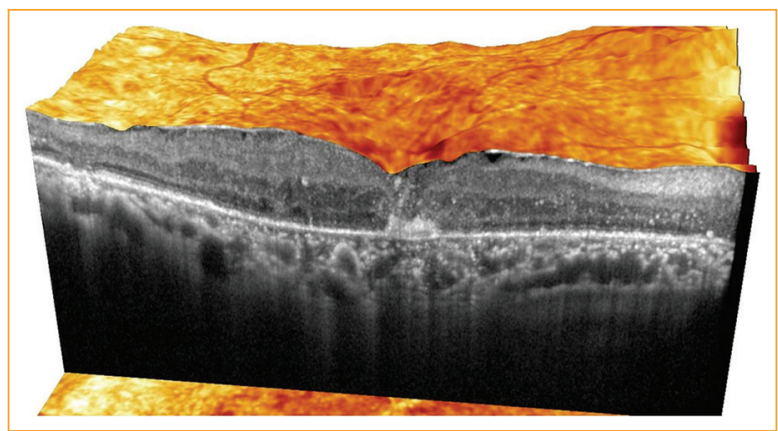

Figura 12. Imagen tomográfica que muestra la evaluación de la coroides usando la estrategia de imagen de profundidad mejorada.

\section{Análisis de volumen macular}

Además del mapa de grosor de la retina, la SD-OCT calcula también el volumen de la retina para cada mapa o para toda la retina (figura 10). Esta tecnología se usa con frecuencia para estudiar los efectos de las diversas intervenciones farmacológicas; sin embargo, al evaluar toda la mácula pueden infraestimarse algunos valores. Se han considerado $\leq 6.5 \pm 1 \mathrm{~mm}^{3} \mathrm{o} \geq$ $8.0 \mathrm{~mm}^{3}$ como valores anormales para el volumen en el área central y pericentral.

\section{Protocolo de análisis del espesor del mapa retiniano}

El mapa retiniano permite determinar la localización $y$ el grosor del edema en nueve sectores maculares dentro de tres círculos concéntricos $(1.3$ y $6 \mathrm{~mm})$ definidos por el Early Treatment Diabetic Retinopathy Study (ETDRS), y evaluar la localización y la distancia del engrosamiento macular con respecto a la fóvea. Además, el protocolo rápido del mapa de espesor macular (fast macular thickness map protocol) combina 
varios mapas de espesor en un par de segundos, permitiendo evaluar los cambios de espesor (medidos en $\mu \mathrm{m}$ ) durante el seguimiento de un paciente con terapia farmacológica y quirúrgica (figura 11). De acuerdo con el ETDRS, el EMD clínicamente significativo correspondería a un grosor macular $\geq 300 \mu \mathrm{m}$ en el milímetro central $y \geq 320 \mu \mathrm{m}$ en el área del anillo pericentral.

\section{Evaluación de la coroides en el edema macular diabético}

El grosor coroideo macular puede evaluarse mediante programas o equipos que disponen de longitudes de onda más largas para evaluar tejidos más profundos. La SD-OCT con imagen de profundidad mejorada es una modificación de la técnica estándar que aprovecha la mayor profundidad del campo, obteniendo una imagen invertida al colocar el tomógrafo más cerca del ojo. Recientemente, la SD-OCT que usa una fuente de barrido posee una mayor longitud de onda (1050 nm) para lograr una mayor profundidad en la exploración macular, así como también una mayor resolución de los tejidos $(5 \mu \mathrm{m})$ (figura 12). Esto cobra relevancia en diversas patologías; por ejemplo, se reporta que existiría un mayor grosor coroideo en pacientes diabéticos en comparación con sujetos sanos ${ }^{11}$.

\section{Bibliografía}

1. Lang E. Optical coherence tomography findings in diabetic retinopathy. Dev Ophthalmol. 2007;39:31-47.

2. Panozzo G, Parolini B, Gusson E, Mercanti A, Pinackatt S, Bertoldo G, et al. Diabetic macular edema: an OCT-based classification. Semin Ophthalmol. 2004;19:13-20.

3. Gerendas B, Prager D, Deak S, Simader C, Lammer J, Wladstein S. Predictive imaging biomarkers relevant for functional and anatomica outcomes during ranibizumab therapy of diabetic macular oedema. $\mathrm{Br} \mathrm{J}$ Ophthalmol. 2018;102:195-203.

4. Deak G, Bolz M, Ritter M, Prager S, Benesch T, Schmidt U. A systematic correlation between morphology and functional alterations in diabetic macular edema. Invest Ophthalmol Vis Sci. 2010;51:6710-14.

5. Gupta A, Raman R, Mohana K, Kulothungan V, Sharma T. Communications between intraretinal and subretinal space on optical coherence tomography of neurosensory retinal detachment in diabetic macular edema. Oman J Ophthalmol. 2013;6:183-8.

6. Sophie R, Lu N, Campochiaro P. Predictor of functional and anatomic outcomes in patients with diabetic macular edema treated with ranibizumab. Ophthalmology. 2015;122:1395-401.

7. Sonoda S, Sakamoto T, Yamashita T, Shirasawa M, Otsuka H, Sonoda Y. Retinal morphologic changes and concentrations of cytokines in eyes with diabetic macular edema. Retina. 2014;34:741-8.

8. Saxena S, Srivastav K, Cheung C, Ngu J, Lai T. Photoreceptor inner segment ellipsoid band integrity on spectral domain optical coherence tomography. Clin Ophthalmol. 2014;8:2507-22.

9. Duker J, Kaiser P, Binder S, de Smet M, Gaudric A, Reichel E, et al. The International Vitreomacular Traction Study Group classification of vitreomacular adhesion, traction and macular hole. Ophthalmology. 2013;120:2611-9.

10. Pai S, Hussain N, Hebri S, Lootah A, Dekhain M. Volcano-like pattern in optical coherence tomography in chronic diabetic macular edema. Saudi J Ophthalmol Soc. 2014;28:157-9.

11. Xu J, Xu L, Du K, Shao L, Chen C, Zhou J, et al. Subfoveal choroidal thickness in diabetes and diabetic retinopathy. Ophthalmology. 2013;120:2023-8. 


\title{
Angiografía por tomografía de coherencia óptica en el edema macular diabético
}

\author{
Juan Manuel Jiménez-Sierra* \\ Asociación para Evitar la Ceguera en México (APEC), Ciudad de México, Méxic
}

La angiografía por tomografía de coherencia óptica (angio-OCT) es un método relativamente nuevo que permite evaluar los vasos de la retina de manera no invasiva, obteniendo imágenes de la circulación de la retina y la coroides en tres dimensiones, sin utilizar colorante alguno ${ }^{1-4}$. El contenido del globo ocular es estático y lo único que se mueve son las células sanguíneas. En este estudio se emplean imágenes de alto contraste con escaneos de muy alta velocidad, y el movimiento es el que genera las imágenes angiográ-

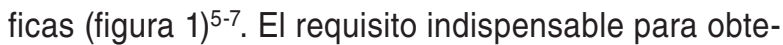
ner las imágenes de los vasos sanguíneos es el movimiento de los eritrocitos; si estos no se mueven o se mueven muy lentamente, no se podrá valorar la luz de los capilares. El principio básico para la OCT y la angio-OCT es la interferometría. La ventaja de este estudio no invasivo es obtener de manera simultánea el estudio de OCT estructural y la angio-OCT, es decir, la estructura vascular-12. Cabe mencionar que este tipo de estudio tiende a presentar artefactos en las imágenes; los más comunes son los de movimiento, visibles como líneas horizontales o verticales blancas, y los de proyección, causados por los vasos o por estructuras más internas sobre los plexos más externos $^{13-16}$.

\section{Definición de segmentación}

En la actualidad, la angio-OCT ofrece la opción de crear angiogramas de $3 \times 3 \mathrm{~mm}, 6 \times 6 \mathrm{~mm}$ y hasta 12 $\times 12 \mathrm{~mm}$. Los nuevos equipos pueden obtener imágenes de campo más amplio, y el futuro es prometedor en ampliar aún más estas dimensiones ${ }^{17-21}$.

En los equipos actuales se puede efectuar la segmentación de la retina por planos, de manera que se obtienen cuatro capas, en las cuales se incluyen los dos plexos vasculares, la capa avascular de la retina (retina externa) y la coriocapilaris (figura 2) $6,7,22,23$.

\section{Nombres de los plexos y su localización ${ }^{12}$}

- Plexo vascular superficial: 3 a $15 \mu \mathrm{m}$ debajo de la membrana limitante interna.

- Plexo capilar profundo: 15 a $70 \mu \mathrm{m}$ debajo de la plexiforme interna.

- Retina externa avascular: $70 \mu \mathrm{m}$ por debajo de la plexiforme externa.

- Coriocapilaris: 30 a $60 \mu \mathrm{m}$ debajo del epitelio pigmentario de la retina.

Al representar los resultados de estos estudios se muestran en forma simultánea las cuatro imágenes de la segmentación: la imagen de angio-flujo y OCT estructural, el mapa de densidad vascular, el grosor de la retina interna, la imagen de oftalmoscopio de escaneo por láser (SLO) y el grosor total de la retina (figura 3) $)^{2,4,24,25}$.

Al mismo tiempo que se observa la segmentación en estas cuatro capas en la angio-OCT, se obtiene una OCT estructural de la retina. De esta forma se pueden valorar simultáneamente anormalidades vasculares de la retina, la estructura de la retina y el grosor central de la retina para valorar el edema macular. Esta 


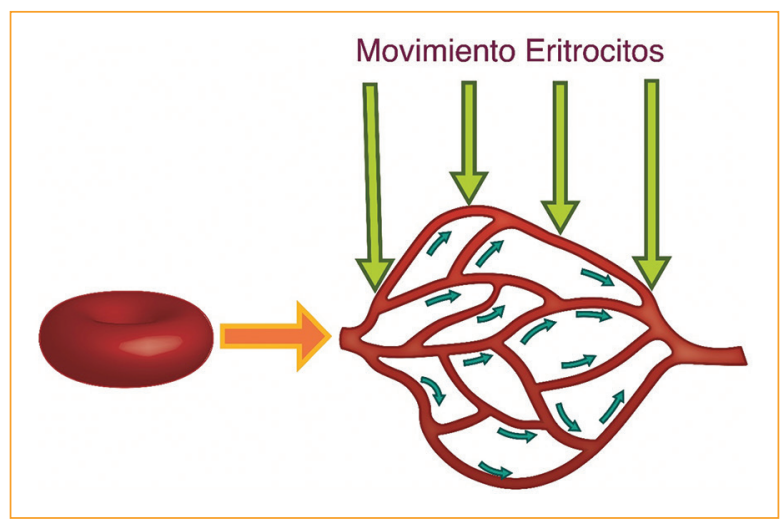

Figura 1. Diagrama que muestra el movimiento de los eritrocitos (flechas) y al hacer múltiples escaneos de alta velocidad se puede dibujar la luz de los vasos y los capilares. (Reproducida con permiso de ref. 25.).

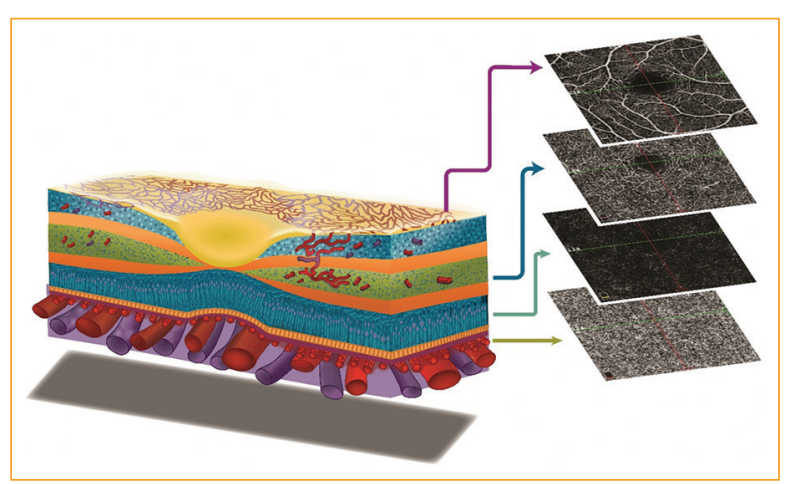

Figura 2. Capas en que se segmenta la retina por angio-0CT. De arriba abajo: plexo vascular superficial, plexo vascular profundo, retina externa avascular y coriocapilaris. (Reproducida con permiso de ref. 25.).

técnica resulta ideal para el diagnóstico y el seguimiento del tratamiento del edema macular diabético (EMD) (figura 4) $)^{24,26-28}$.

Las imágenes de angio-OCT se presentan en dos maneras diferentes:

- Una sobreimposición de las estructuras de flujo, las cuales por lo general se muestran en rojo, sobre un B-scan convencional, donde las estructuras no vasculares se muestran con la reflectividad acostumbrada.

- Imágenes en-face, que muestran un corte coronal, ya sea de OCT o de angio-OCT, en las cuales se observan las estructuras vasculares en una reconstrucción bidimensional y por planos (figura 5) 2,4,24,29.

\section{Plexos vasculares y zona foveal avascular}

Existen dos plexos vasculares en la angio-OCT de la retina:

- Plexo vascular superficial: 3-15 $\mu \mathrm{m}$ debajo de la membrana limitante interna.

- Plexo vascular profundo: $15-70 \mu \mathrm{m}$ debajo de la plexiforme interna.

La zona foveal avascular (ZFA) y la red perifoveal pueden ser valoradas en estos plexos, siendo más fácil hacerlo en el plexo vascular superficial. En la retinopatía diabética y el EMD, las principales alteraciones se observarán en estos plexos ${ }^{22}$.

\section{Parámetros de cuantificación de la densidad vascular y métricas de la zona foveal avascular}

La ZFA (ZAF por sus siglas en inglés) se define como el área central de la retina que está libre de capilares. La manera de cuantificar las medidas de esta área se conoce como métricas de ZFA, y son:

- Área de la ZFA.

- Perímetro de la ZFA.

- Índice de circularidad.

El índice de circularidad corresponde a la relación entre el perímetro medido y el perímetro del área circular del mismo tamaño y densidad vascular parafoveal, que es la densidad de vasos foveales en una región de $300 \mu \mathrm{m}$ de diámetro alrededor de ZFA (FD300) (figura 6) 24-26,29.

Estas medidas han mostrado utilidad para valorar la integridad de la red capilar microvascular en patologías que producen isquemia (isquemia macular diabética) $)^{10-12}$.

La densidad vascular se mide a nivel del plexo superficial (membrana limitante interna). La capa plexiforme interna (IPL - 10) corresponde a la densidad vascular superficial (sDV), y a nivel del plexo profundo (IPL - 10 capa plexiforme externa [OPL] + 10) corresponde a la densidad vascular profunda (pDV). El análisis de densidad vascular (DV) calcula el porcentaje del área ocupada en la angio-OCT en la cual se detecta vasculatura (figura 7). El índice de flujo se calcula como el valor de la señal de flujo en una región de interés. Las áreas de bajo flujo se detectan como zonas anormalmente amplias de bajo pixelaje en los angiogramas donde deberían encontrarse niveles más altos de densidad vascular. Esto también puede ser representado en una «paleta de color», en la cual los colores 


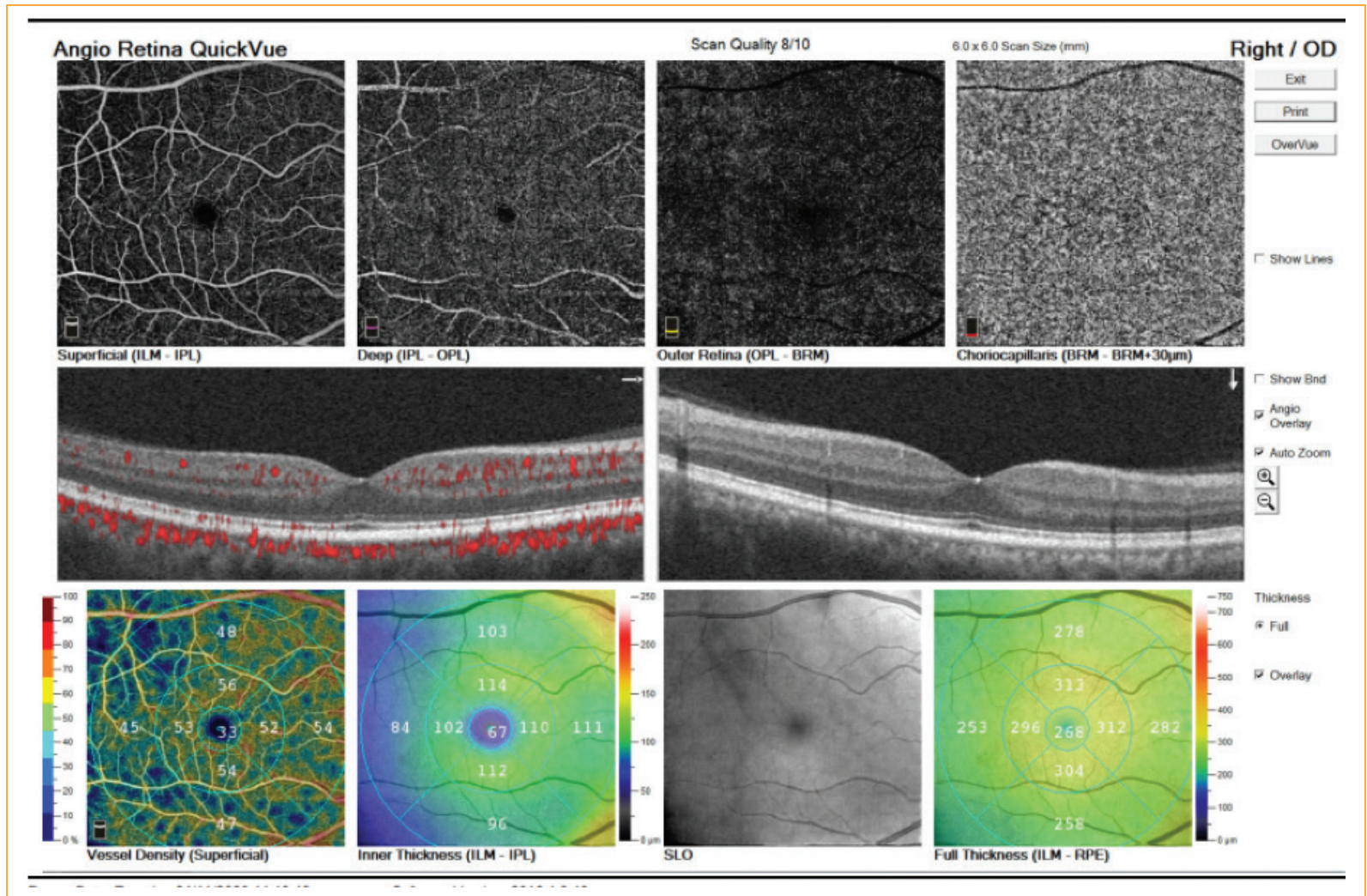

Figura 3. Estudio de angio-OCT de un paciente diabético de 43 años sin retinopatía diabética (estudio normal). En las fotografías superiores, de izquierda a derecha se observan el plexo capilar superficial, el plexo capilar profundo, la retina externa avascular y los coriocapilares normales. En la parte media se muestran imágenes de angio-flujo y OCT estructural normales. En las imágenes inferiores podemos ver el mapa de densidad vascular superficial (con su paleta de colores del lado izquierdo), el grosor de la retina interna ILM-IPL (con su correspondiente paleta de colores del lado derecho), la imagen de SLO y el mapa del grosor total de la retina (con su correspondiente paleta de colores del lado derecho).

calientes corresponden a una mayor densidad vascular y los colores fríos a una menor densidad vascular ${ }^{25,26}$.

\section{Retinopatía diabética}

La aplicación de la angio-OCT en el diagnóstico y el seguimiento de la retinopatía diabética ha escalado recientemente de manera incesante. La nueva perspectiva de evaluación que ofrece la angio-OCT aporta un enfoque diferente y complementario al que se podía obtener con la angiografía fluoresceínica o el B-scan de la OCT.

Desde el punto de vista cualitativo, la angio-OCT ha demostrado ser comparable a la angiografía fluoresceínica en la detección de neovasos, incluso a pesar del inconveniente que puede representar el limitado campo de adquisición (figura 8) ${ }^{17}$.

Por otra parte, la angio-OCT es superior a la angiografía fluoresceínica en la detección de anormalidades microvasculares intrarretinianas (IRMA, intraretinal microvascular abnormalities) y en la cuantificación de las áreas de no perfusión capilar (figura 9). La angioOCT puede detectar hasta un $40 \%$ más lesiones tipo IRMA que la foto a color. Sin embargo, se ha reportado que la detección de microaneurismas puede ser hasta un $40 \%$ inferior que en la angiografía fluoresceínica, probablemente debido al lento flujo que pueden tener este tipo de lesiones. Pese a ello, la angio-OCT tiene la ventaja de poder discriminar la localización de los microaneurismas por plexo, lo que podría tener un valor predictivo en el futuro (figura 9) ${ }^{13,28,29}$.

Probablemente una de las principales utilidades que tiene la angio-OCT en la evaluación del paciente diabético es la detección de la maculopatía isquémica diabética. La alta resolución de esta tecnología permite identificar la pérdida de la integridad del anillo anastomótico, así como las áreas de pérdida de capilares o 
Tabla 1. Relación entre la métrica de la zona foveal avascular y la gravedad de la retinopatía

\begin{tabular}{|l|l|l|}
\hline Métrica & Correlación & Descripción \\
\hline Área de la ZFA & Directa & A mayor gravedad de la retinopatía diabética, mayor área de la ZFA \\
\hline Índice de circularidad & Directa & A mayor gravedad de la retinopatía diabética, mayor es el índice de circularidad de la ZFA \\
\hline Densidad vascular & Inversa & A mayor gravedad de la retinopatía diabética, menor es el porcentaje de densidad vascular
\end{tabular}

ZFA: zona foveal avascular.

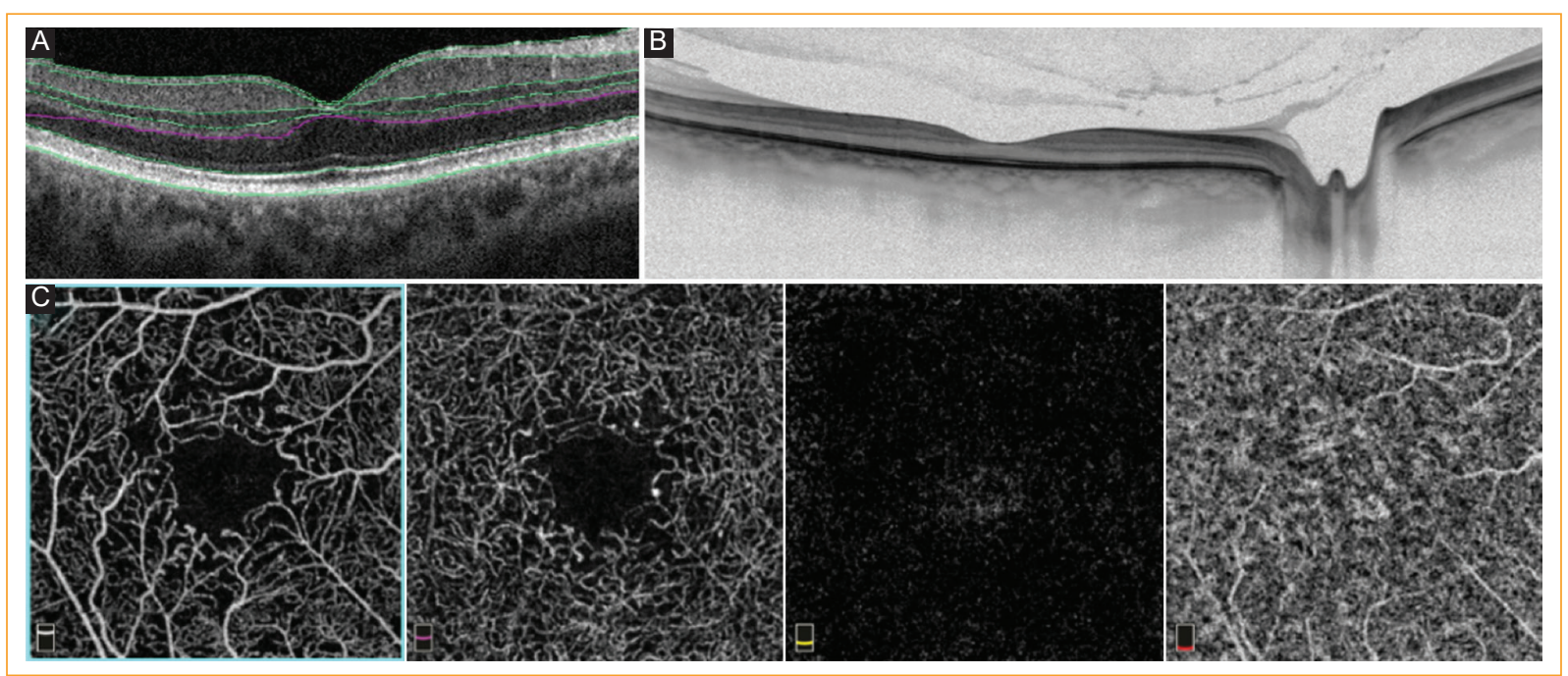

Figura 4. Paciente diabético con microaneurismas visibles en los plexos superficial y profundo. A: scan de alta resolución en el cual se observan las diferentes capas de la retina. B: scan con delimitación de las diferentes capas de la retina. La segmentación en ocho diferentes capas es posible con el uso de los softwares modernos, y además se puede realizar un ajuste manual para evitar artefactos de segmentación. C: angiograma correspondiente a los plexos superficial y profundo, la retina externa avascular y la coriocapilaris (de izquierda a derecha). Nótese que los vasos siguen la misma distribución de los grandes vasos en el plexo superficial. (Reproducida con permiso de ref. 25.).

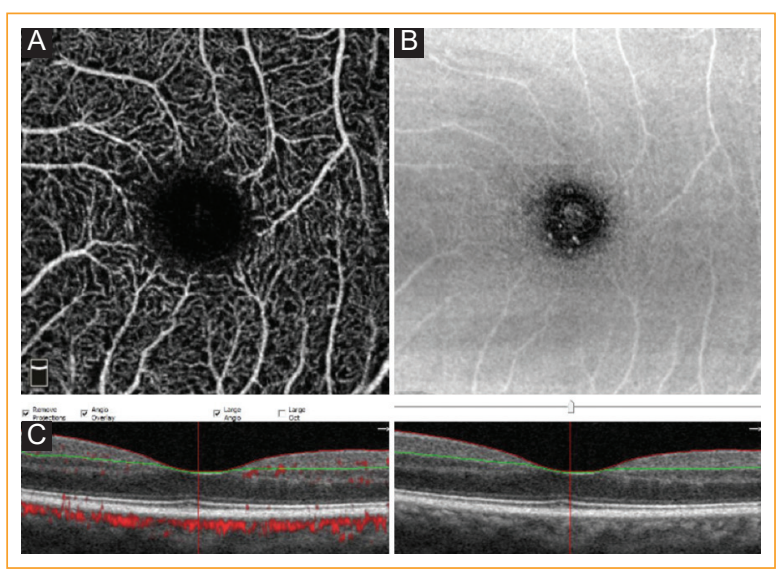

Figura 5. A: imagen en face angio-OCT del plexo superficial. B: imagen en face de OCT. C: imagen de corte transversal con visualización de las estructuras que presentan flujo vascular marcadas en rojo. (Reproducida con permiso de ref. 25.). de muy bajo flujo vascular yuxtafoveales, aun en presencia de EMD (figuras 10 y 11) 11,12,29.

La principal ventaja de la angio-OCT frente a la angiografía fluoresceínica en los pacientes diabéticos es poder realizar un análisis cuantitativo del componente vascular ${ }^{24}$.

Diversos artículos han reportado que en los pacientes diabéticos existe una correlación del aumento del área de la ZFA, la pérdida de la circularidad de la ZFA y la disminución del porcentaje de densidad vascular perifoveal con la gravedad de la retinopatía diabética (tabla 1) 24,25 .

Algunos autores han observado cambios tempranos en la métrica de la ZFA en pacientes diabéticos sin retinopatía diabética, lo que sugiere que el daño vascular puede preceder al daño estructural y predecir la progresión de la diabetes aun en ausencia de cambios anatómicos. De 


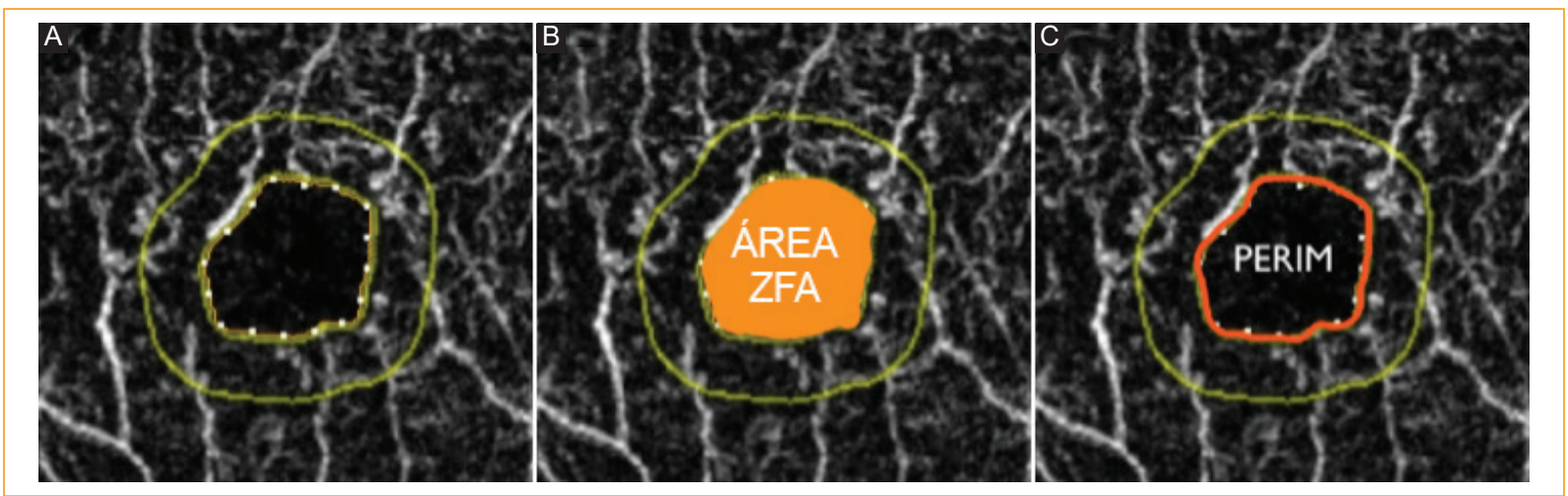

Figura 6. A: zona foveal avascular (ZFA) medida en un corte de retina completo de manera automática. B: área de la ZFA. C: perímetro mostrado por la línea roja. (Reproducida con permiso de ref. 25.).
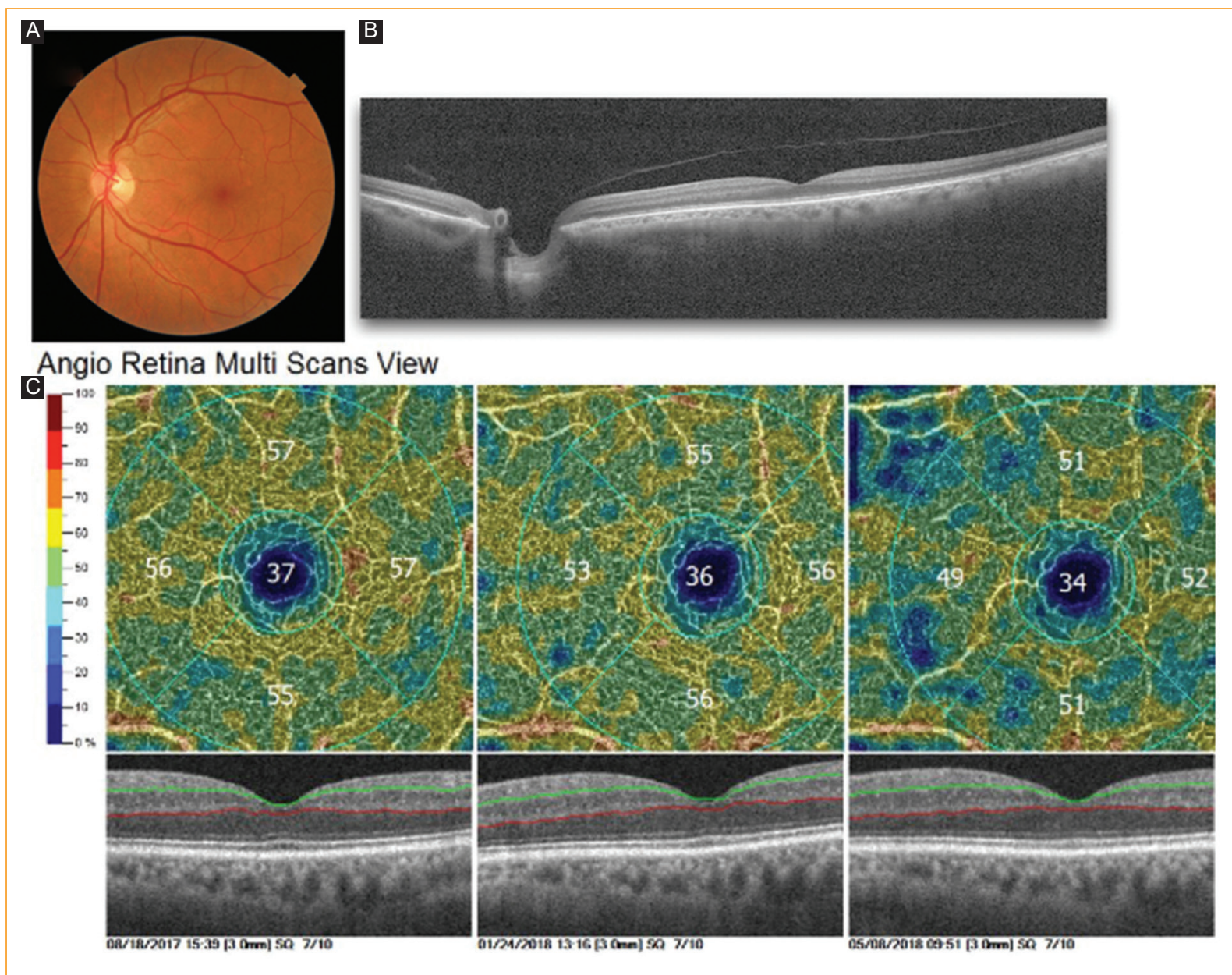

Figura 7. A: fotografía del polo posterior. B: imagen de B-scan de un paciente diabético sin signos de retinopatía diabética. C: mapa de densidad vascular. El seguimiento cuantitativo con angio-OCT en visitas consecutivas del mismo paciente muestra cambios de disminución en el porcentaje de densidad vascular, lo que sugiere una progresión de la enfermedad. (Reproducida con permiso de ref. 25.). 


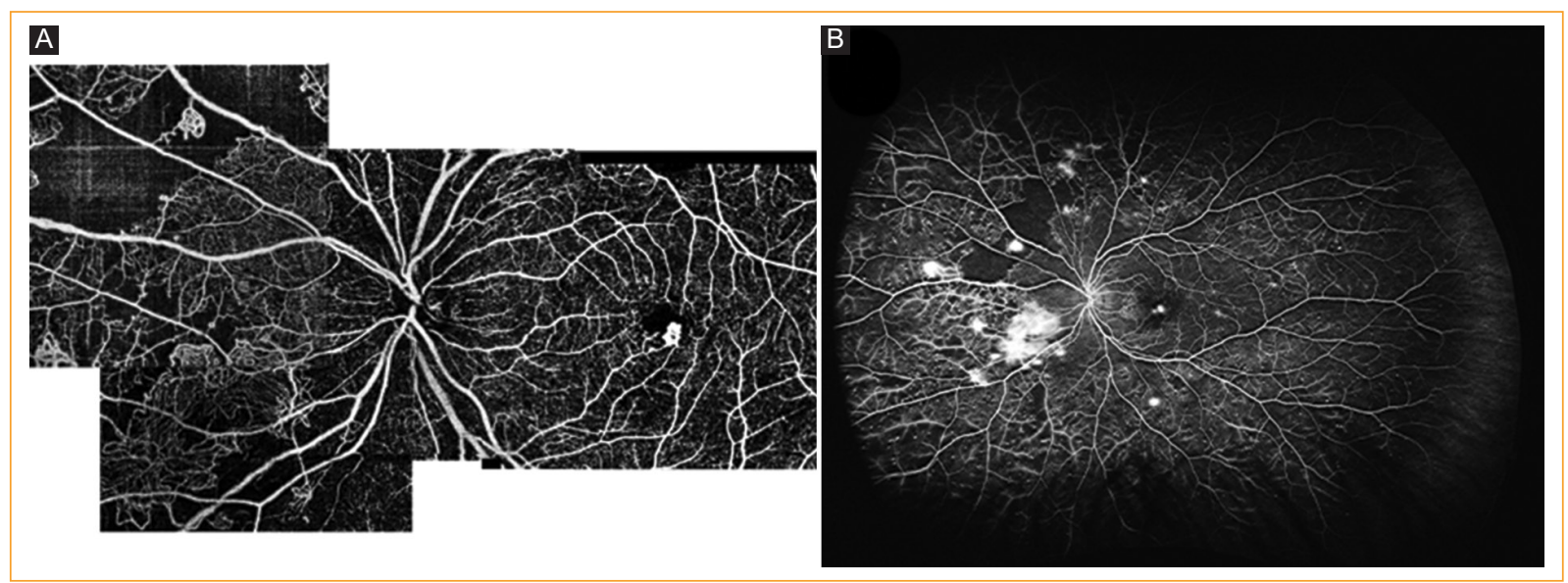

Figura 8. A: composición en mosaico de un paciente con retinopatía diabética proliferativa. La angio-OCT detecta la presencia de neovasos en el área yuxtafoveal y en el sector nasal al disco. La construcción de los mosaicos puede ampliar el área de evaluación en pacientes con vasculopatías que no se limitan al área macular, como la retinopatía diabética. B: angiografía fluoresceínica de campo amplio del mismo paciente. Se puede observar que la imagen no invasiva obtenida con angio-OCT ofrece un perfil diagnóstico y de caracterización comparable. (Reproducida con permiso de ref. 25.).

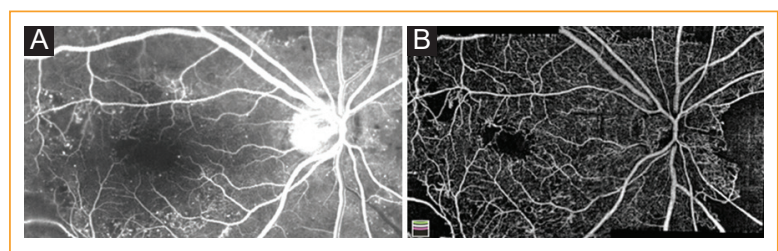

Figura 9. A: angiografía fluoresceínica. B: angio-OCT de un paciente con retinopatía diabética no proliferativa grave. Si bien las imágenes son comparables en términos de capacidad diagnóstica, la resolución y el nivel de detalle de la angio-OCT permiten identificar las áreas de no perfusión capilar o de muy bajo flujo con mayor contraste y mejor definición. (Reproducida con permiso de ref. 25.).

ahí que el seguimiento cuantitativo longitudinal del paciente diabético con angio-OCT podría permitir un análisis personalizado del control de su enfermedad ${ }^{24}$.

A continuación se presentan las ventajas y las desventajas de la angiografía de retina con fluoresceína y la angio-OCT en el EMD.

\section{Angiografía con fluoresceína}

- Ventajas: detecta áreas de cierre capilar, áreas de neovascularización, IRMA, la localización de la mayoría de los microaneurismas y capilares anormales con escape de colorante. Con los equipos de campo amplio y ultraamplio pueden detectarse áreas de cierre capilar periféricas, así como áreas de neovasos periféricas ${ }^{15,25}$.

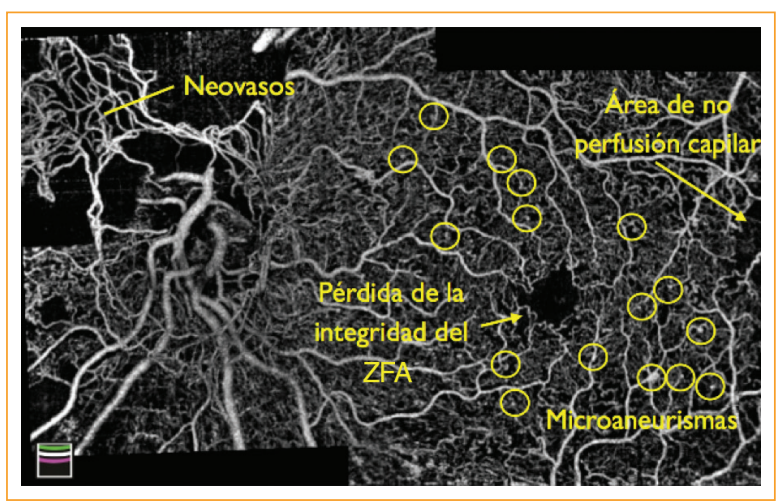

Figura 10. Capacidad de la angio-OCT para identificar lesiones características de la retinopatía diabética. Los microaneurismas se encuentran en los círculos amarillos. La zona foveal avascular (ZFA) es irregular. Se observan áreas de cierre capilar y presencia de neovasos en la papila. (Reproducida con permiso de ref. 25.).

- Desventajas: es un método invasivo con riesgo de algunos efectos secundarios, como náusea, vómito, reacción alérgica, broncoespasmo y anafilaxia, y riesgo de muerte reportado de 1 en 10,000 a 1 en 200,000 . Para la angiografía de retina se requieren un equipo fotográfico (cámara de fondo de ojo) y un técnico especializado en el estudio, y su duración aproximada es de 15-20 minutos tras la dilatación de la pupila. En el EMD este estudio no se repite con frecuencia, ya que el seguimiento se sugiere con 


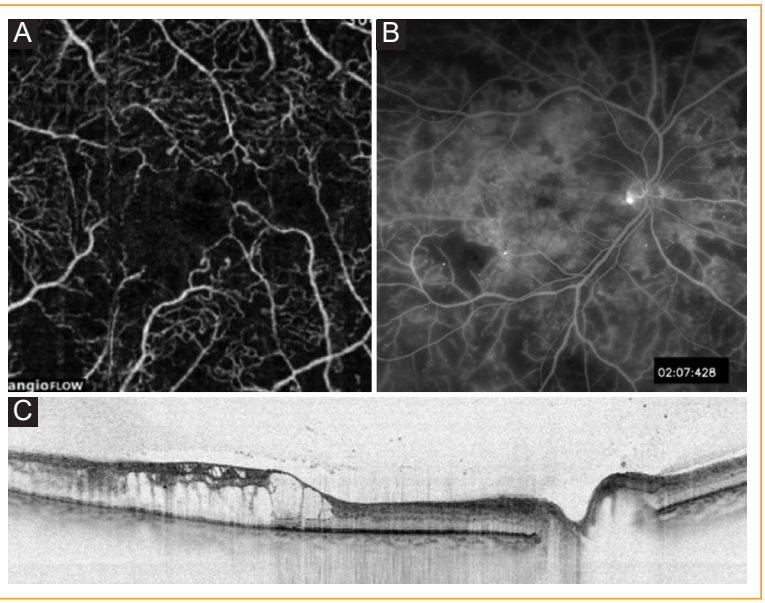

Figura 11. A: angiografía fluoresceínica. B: angio-0CT $3 \times$ $3 \mathrm{~mm}$. C: B-scan de un paciente con edema macular diabético grave. Se observa que el fenómeno de escape de la angiografía fluoresceínica constituye una limitación para evaluar adecuadamente la integridad de la zona foveal avascular. En cambio, la angio-OCT muestra claramente la pérdida de la integridad del anillo anastomótico de la zona foveal avascular. (Reproducida con permiso de ref. 25.).

OCT. Se debe tener un equipo para resolver posibles reacciones alérgicas ${ }^{15,25}$.

\section{Angio-OCT en el edema macular diabético}

- Ventajas: es un estudio no invasivo, sin riesgos, que se puede repetir para el seguimiento del EMD cada vez que se efectúe una OCT estructural. Puede valorar proliferaciones y cerca del $40 \%$ de los microaneurismas, así como la presencia de vasos anormales y áreas de cierre capilar. El tiempo por escaneo es de 10 segundos. El resultado puede valorarse de inmediato y es altamente comparativo entre estudios. No se requiere dilatación de la pupila.

- Desventajas: los equipos actuales no valoran la retina periférica ni el escape de colorante. Se requiere el movimiento de los eritrocitos dentro de los capilares para detectar los vasos (pueden perderse algunos capilares si el flujo es muy lento). Son necesarios un equipo de angio-OCT y un operador entrenado.

\section{Bibliografía}

1. De Carlo T, Romano A, Waheed N, Duker J. A review of optical coherence tomography angiography (OCTA). Int $\mathrm{J}$ Retina Vitreous. 2015;15:1-5.

2. Schwartz D, Fingler J, Kim D, Zawadzki R, Morse L, Park S, et al. Phase-variance optical coherence tomography: a technique for noninvasive angiography. Ophthalmology. 2014;121:180-7.

3. Turgut B. Optical coherence tomography angiography - a general view. Eur Ophthalmic Rev. 2016;10:39.

4. Campbell J, Zhang M, Hwang T, Bailey S, Wilson D, Jia Y, et al. Detailed vascular anatomy of the human retina by projection-resolved optical coherence tomography angiography. Sci Rep. 2017;10:42201.
5. Yu P, Balaratnasingam C, Cringle S, McAllister I, Provis J, Yu D. Microstructure and network organization of the microvasculature in the human macula. Invest Ophthalmol Vis Sci. 2010;51:6735-43.

6. Waheed N, Moult E, Fujimoto J, Rosenfeld P. Optical coherence tomography angiography of dry age-related macular degeneration. Dev Ophthalmol. 2016;15:91-100.

7. De Oliveira J, Zhang Q, Garcia J, Zheng F, Motulsky E, Roisman L, et al. Natural history of subclinical neovascularization in nonexudative age-related macular degeneration using swept-source OCT angiography. Ophthalmology. 2018;125:255-66.

8. Miller A, Roisman L, Zhang Q, Zheng F, de Oliveira R, Yehoshua Z, et al Comparison between spectral-domain and swept-source optical coherence tomography angiographic imaging of choroidal neovascularization. Invest Ophthalmol Vis Sci. 2017;58:1499-505.

9. Azar G, Wolff B, Mauget M, Rispoli M, Savastano M, Lumbroso B. Pachychoroid neovasculopathy: aspect on optical coherence tomography angiography. Acta Ophthalmol. 2017;95:421-7.

10. Powner M, Sim D, Zhu M, Nobre J, Jones R, Syed A, et al. Evaluation of nonperfused retinal vessels in ischemic retinopathy. Invest Ophthalmol Vis Sci. 2016;57:5031-7.

11. Singh A, Agarwal A, Mahajan S, Karkhur S, Singh R, Bansal R, et al. Morphological differences between optic disc collaterals and neovascularization on optical coherence tomography angiography. Graefes Arch Clin Exp Ophthalmol. 2017;255:753-9.

12. Suzuki N, Hirano $Y$, Tomiyasu $T$, Esaki $Y$, Uemura A, Yasukawa $T$, et al. Retinal hemodynamics seen on optical coherence tomography angiography before and after treatment of retinal vein occlusion. Invest Ophthalmol Vis Sci. 2016:57:5681-7.

13. Stitt A, Gardiner T, Archer D. Histological and ultrastructural investigation of retinal microaneurysm development in diabetic patients. Br J Ophthalmol. 1995;79:362-7.

14. Spaide F, Klancnik J, Cooney M, Yannuzzi L, Balaratnasingam C, Dansingani K, et al. Volume-rendering optical coherence tomography angiography of macular telangiectasia type 2. Ophthalmology. 2015;122:2261-9.

15. Lumbroso B, Huang D, Chen $C$, Jia Y, Rispoli M, Romano A, et al. Clinical OCT angiography atlas. JP Medical Ltd; 2015. 182 p.

16. Spaide R, Klancnik J, Cooney M. Retinal vascular layers in macular telangiectasia type 2 imaged by optical coherence tomographic angiography. JAMA Ophthalmol. 2015:133:66-73.

17. Nesper P, Roberts P, Onishi A, Chai H, Liu L, Jampol L, et al. Quantifying microvascular abnormalities with increasing severity of diabetic retinopathy using optical coherence tomography angiography. Invest Ophthalmol Vis Sci. 2017:58:307-15.

18. Ishibazawa A, Nagaoka T, Yokota H, Takahashi A, Omae T, Song Y, et al. Characteristics of retinal neovascularization in proliferative diabetic retinopathy imaged by optical coherence tomography angiography. Invest Ophthalmol Vis Sci. 2016;57:6247-55

19. Stanga P, Papayannis A, Tsamis E, Stringa F, Cole T, D'Souza Y, et al. New findings in diabetic maculopathy and proliferative disease by swept-source optical coherence tomography angiography. Dev Ophthalmol. 2016;56:113-21

20. Rispoli M, Savastano M, Lumbroso B. Capillary network anomalies in branch retinal vein occlusion on optical coherence tomography angiography. Retina. 2015;35:2332-8.

21. Adhi M, Bonini Filho M, Louzada R, Kuehlewein L, de Carlo T, Baumal C, et al. Retinal capillary network and foveal avascular zone in eyes with vein occlusion and fellow eyes analyzed with optical coherence tomography angiography. Invest Ophthalmol Vis Sci. 2016;57:486.

22. Couturier A, Mané V, Bonnin S, Erginay A, Massin P, Gaudric A, et al. Capillary plexus anomalies in diabetic retinopathy on optical coherence tomography angiography. Retina. 2015;35:2384-91.

23. Tang F, Ng D, Lam A, Luk F, Wong R, Chan C, et al. Determinants of quantitative optical coherence tomography angiography metrics in patients with diabetes. Sci Rep. 2017;7:2575.

24. Dimitrova G, Chihara E, Takahashi H, Amano H, Okazaki K. Quantitative retinal optical coherence tomography angiography in patients with diabetes without diabetic retinopathy. Invest Ophthalmol Vis Sci. 2017:58:190-6.

25. Acosta C, Sardi C, Sánchez J. En: Manual de métodos diagnósticos en oftalmología. Jiménez-Sierra JM: APEC; 2019.

26. Hwang T, Gao S, Liu L, Lauer A, Bailey S, Flaxel C, et al. Automated quantification of capillary nonperfusion using optical coherence tomography angiography in diabetic retinopathy. JAMA Ophthalmol. 2016;134:367.

27. Schaal K, Munk M, Wyssmueller I, Berger L, Zinkernagel M, Wolf S. Vascular abnormalities in diabetic retinopathy assessed with swept-source optical coherence tomography angiography wide field imaging. Retina. 2019;39:79-87.

28. Wang H, Chablani J, Freeman W, Chan C, Kozak I, Bartsch D, et al. Characterization of diabetic microaneurysms by simultaneous fluorescein angiography and spectral-domain optical coherence tomography. Am J Ophthalmol. 2012;153:861-7.

29. Suzuki N, Hirano $Y$, Yoshida M, Tomiyasu $T$, Uemura A, Yasukawa $T$, et al. Microvascular abnormalities on optical coherence tomography angiography in macular edema associated with branch retinal vein occlusion. Am J Ophthalmol. 2016;161:126-32. 


\title{
Láser en el edema macular diabético
}

\author{
Gerardo García-Aguirre* \\ Asociación para Evitar la Ceguera en México (APEC), Ciudad de México, México
}

La fotocoagulación con láser fue uno de los primeros tratamientos en demostrar su eficacia en el manejo del edema macular diabético. En 1991, el Early Treatment Diabetic Retinopathy Study (ETDRS) reportó que la aplicación de fotocoagulación con láser focal (argón, longitud de onda de $532 \mathrm{~nm}$ ) disminuía significativamente $(50 \%)$ el riesgo de pérdida visual moderada en pacientes con edema macular clínicamente significativo $0^{1,2}$.

\section{Técnica de aplicación de fotocoagulación selectiva}

El objetivo del tratamiento era la creación de quemaduras directamente en los microaneurismas que fugaban, evidenciados por angiografía con fluoresceína (fotocoagulación ETDRS focal dirigido) o la aplicación generalizada de fotocoagulación a las zonas de fuga difusa (fotocoagulación ETDRS focal en parrilla). Las características de cada una de estas técnicas se describen en la tabla 1.

En la práctica clínica hay una correlación directa entre la quemadura directa de los aneurismas que fugan y la reducción en el grosor macular (figura 1).

Entre los principales inconvenientes del tratamiento con láser según el ETDRS, ya sea dirigido o en parrilla, están el daño irreversible que causan las quemaduras de la retina en un área tan sensible y el crecimiento de las cicatrices del láser (y de los escotomas asociados) con el paso de los años (figura 2). Si este crecimiento llega a involucrar el centro de la fóvea, puede causar una pérdida grave e irreversible de la visión.
Tomando en cuenta estos inconvenientes, se postuló el uso de una técnica modificada que resultara más gentil para la mácula, denominada «parrilla macular leve» (mild macular grid) (tabla 1), en la que se hacían quemaduras más leves dispersas por toda la mácula, independientemente de las áreas de engrosamiento. Esta técnica resultó ser menos eficaz que la parrilla macular tradicional para reducir el grosor macular medido por tomografía de coherencia óptica, sin que hubiera una diferencia significativa en la agudeza visual entre los dos tratamientos ${ }^{3}$.

\section{Longitud de onda}

Si bien el ETDRS se realizó utilizando láser de argón de $532 \mathrm{~nm}$, por ser la opción prevalente en la época en que se hizo el estudio, hoy en día es aceptable utilizar láseres de cualquiera de las longitudes de onda usadas para la fotocoagulación de retina $(532 \mathrm{~nm}$ verde Nd: YAG de onda duplicada, $577 \mathrm{~nm}$ amarillo, $810 \mathrm{~nm}$ infrarrojo).

\section{Uso actual de la fotocoagulación selectiva}

Desde la aparición de los fármacos dirigidos contra el factor de crecimiento endotelial vascular (anti-VEGF [vascular endothelial growth factor]), el uso de la fotocoagulación selectiva ha disminuido significativamente, debido a la mayor eficacia de dichos fármacos. Esto fue evidenciado desde los estudios iniciales que compararon los anti-VEGF con el láser (el primero fue el ranibizumab, en el estudio RESTORE), y se hizo más 
Tabla 1. Parámetros para fotocoagulación selectiva según el ETDRS

\begin{tabular}{|l|l|l|l}
\hline & Focal dirigido & Focal en parrilla & Parrilla macular leve \\
\hline Tamaño de la quemadura & $50-100 \mu \mathrm{m}$ & $<200 \mu \mathrm{m}$ & $50 \mu \mathrm{m}$ \\
\hline Tiempo de exposición & $50-100 \mathrm{~ms}$ & $50-100 \mathrm{~ms}$ & $50-100 \mathrm{~ms}$ \\
\hline Intensidad & $\begin{array}{l}\text { Blanqueamiento u oscurecimiento } \\
\text { de microaneurismas }\end{array}$ & Leve & Leve \\
\hline Número de quemaduras & $\begin{array}{l}\text { Suficiente para tratar las zonas } \\
\text { de fuga focal }\end{array}$ & $\begin{array}{l}\text { Suficiente para cubrir las } \\
\text { áreas de fuga difusa }\end{array}$ & $\begin{array}{l}200-300, \text { cubriendo toda el área } \\
\text { macular independientemente del } \\
\text { engrosamiento }\end{array}$ \\
\hline Localización & $\begin{array}{l}500-3000 \mu \mathrm{m} \text { del centro de la } \\
\text { fóvea }\end{array}$ & $\begin{array}{l}500-3000 \mu m \text { del centro de la } \\
\text { fóvea }\end{array}$ & $500-3000 \mu$ del centro de la fóvea \\
\hline
\end{tabular}
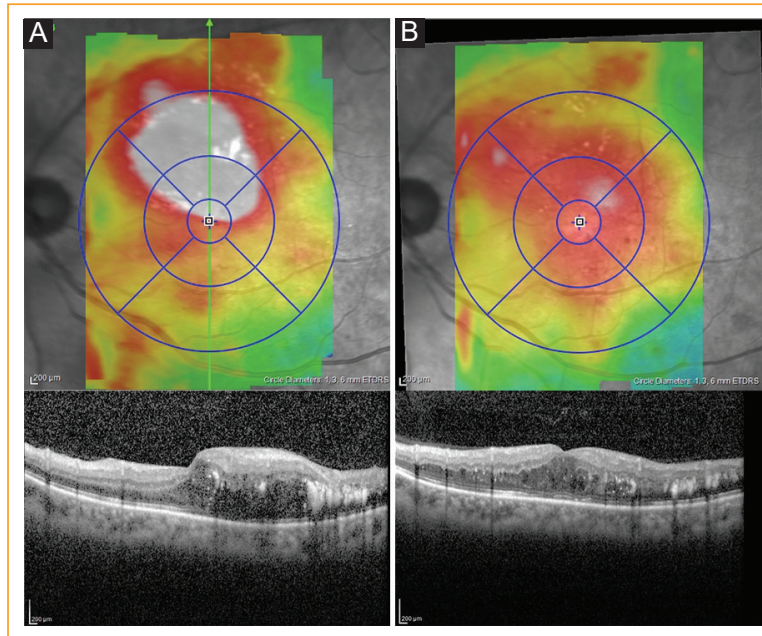

Figura 1. Grosor macular antes y después del tratamiento con fotocoagulación selectiva focal. A: estudio pretratamiento. Se observa un marcado engrosamiento retiniano, en especial en la porción superior de la mácula, evidenciado también en el corte vertical de tomografía de coherencia óptica. B: estudio postratamiento. Se observan una reducción importante en el grosor retiniano después de tratar los microaneurismas que fugaban y la persistencia de líquido intrarretiniano, que requirió inyecciones intravítreas de anti-VEGF.

evidente en estudios como el protocolo I del DRCR. net, en el que los ojos tratados con fotocoagulación selectiva macular tuvieron agudezas visuales significativamente menores y tardaron mucho más tiempo en la reducción del edema en comparación con los ojos tratados con ranibizumab. Además, los ojos tratados con ranibizumab y láser selectivo desde el principio tuvieron menor agudeza visual en un seguimiento a 5 años que los ojos que solo recibieron ranibizumab ${ }^{4,5}$.
En el edema macular focal sin involucro central de la mácula, la fotocoagulación selectiva puede ser una excelente opción. Si el edema involucra la parte central de la mácula o existen microaneurismas en las $300 \mu \mathrm{m}$ centrales, se sugiere terapia anti-VEGF y láser solo de rescate. Para valorar la respuesta del edema macular después de la fotocoagulación selectiva es necesario esperar un mínimo de 4 meses.

\section{Láser micropulsado o subumbral}

Recientemente, el láser micropulsado o subumbral se ha agregado al arsenal terapéutico contra el edema macular. El tratamiento consiste en aplicar láser a la retina con altos poderes y cortos pulsos (micropulsado), o con bajo tiempo de exposición (subumbral), insuficientes para elevar de manera sustancial la temperatura de la retina y, por lo tanto, incapaces de causar una quemadura visible. Este tratamiento, dado su efecto térmico leve ejercido especialmente sobre las células del epitelio pigmentado de la retina (al parecer estimulando las proteínas de choque térmico), induce la absorción de líquido intrarretiniano y subretiniano (figura 3$)^{6-8}$.

Para aplicar el láser micropulsado se recomienda un diámetro de disparo de $200 \mu \mathrm{m}$. Para ajustar el poder se sugiere realizar disparos de prueba por fuera de la mácula hasta alcanzar una marca visible de color blanco-grisáceo. Luego se multiplica el poder por 4 (p. ej., si se requirieron $150 \mathrm{~mW}$ para causar una quemadura visible, el poder para el láser micropulsado será de 600 $\mathrm{mW}$ ) y se activa la opción de láser micropulsado con un ciclo de trabajo del $5 \%$, y se aplica el tratamiento con disparos confluentes en toda el área macular. Como no hay marcas visibles con el tratamiento, es de 


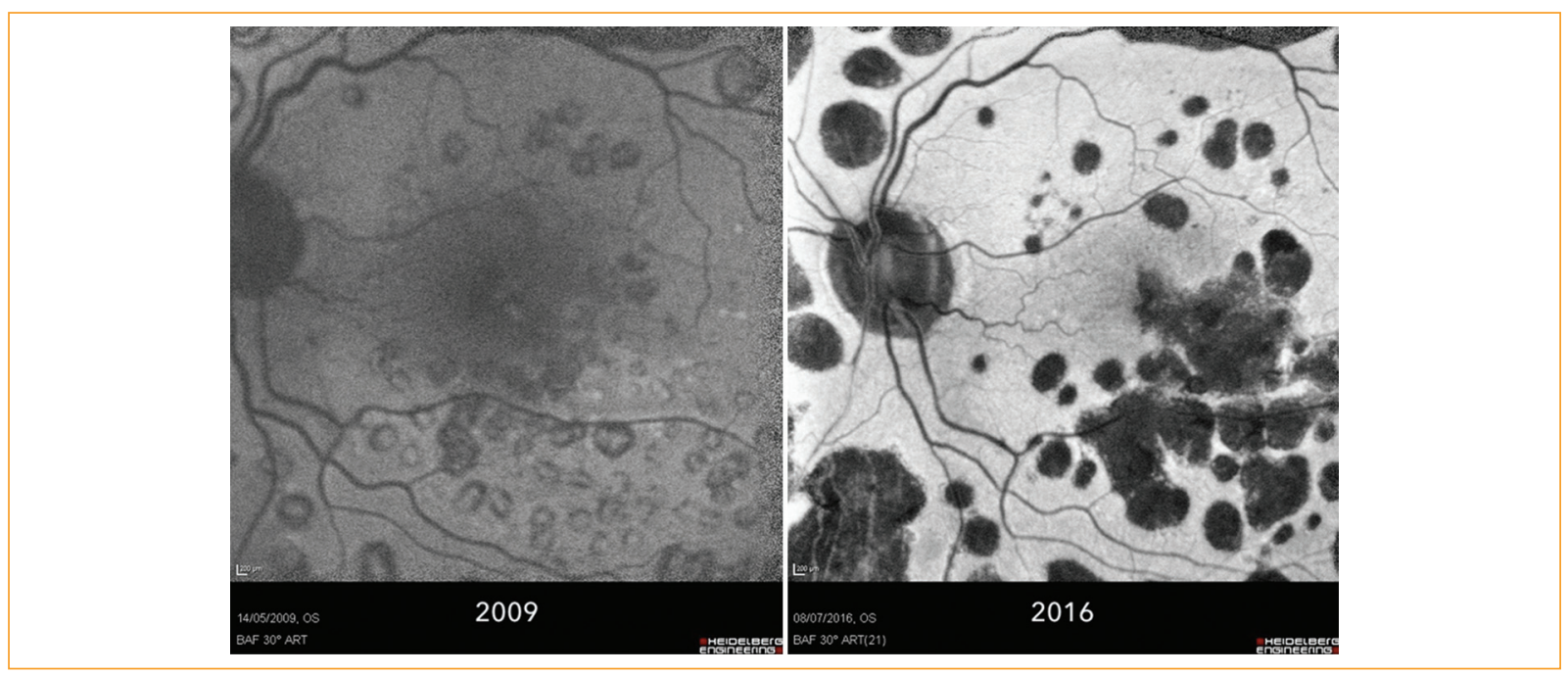

Figura 2. Imágenes de autofluorescencia que muestran el crecimiento de las huellas de fotocoagulación focal selectiva después de 7 años.

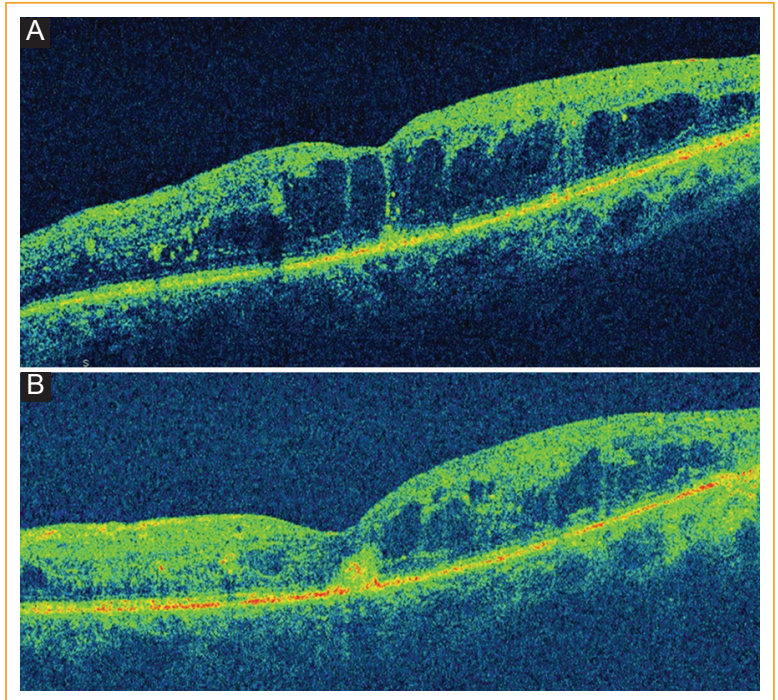

Figura 3. Tomografía de coherencia óptica macular de un ojo con edema macular diabético que involucra el centro macular. A: después de ocho inyecciones intravítreas de bevacizumab, agudeza visual 20/80, paciente catalogado como parcialmente respondedor. B: después del tratamiento con láser micropulsado y dos inyecciones intravítreas más de bevacizumab, importante reducción del edema.

gran ayuda tener un sistema de rejilla automatizada que aplique los disparos sobre un área determinada.

Respecto a la eficacia, cuando se ha comparado el láser micropulsado con la fotocoagulación selectiva convencional se han encontrado efectos similares en cuanto a agudeza visual y reducción del grosor macular, con menor afección de la sensibilidad al contraste. Aunque se han realizado estos estudios, aún no hay evidencia suficiente para saber exactamente cuál es el papel de esta modalidad de tratamiento en el manejo del edema macular diabético.

\section{Bibliografía}

1. Early Treatment Diabetic Retinopathy Study Research Group. Early photocoagulation for diabetic retinopathy. ETDRS Report number 9. Ophthalmology. 1991;98:766-85.

2. Early Treatment Diabetic Retinopathy Study Research Group. Treatment techniques and clinical guidelines for photocoagulation of diabetic macular edema. ETDRS Report number 2. Ophthalmology. 1987;94: 761-74.

3. Writing Committee for the Diabetic Retinopathy Clinical Research Network. Comparison of the modified Early Treatment Diabetic Retinopathy Study and mild macular grid laser photocoagulation strategies for diabetic macular edema. Arch Ophthalmol. 2007;125:469-80.

4. Schmidt U, Lang G, Holz F, Schlingemann R, Lanzetta P, Massin P, et al. Three-year outcomes of individualized ranibizumab treatment in patients with diabetic macular edema. Ophthalmology. 2014;121: 1045-53.

5. Elman M, Bressler N, Qin H, Beck R, Ferris F, Friedman S, et al. Expanded 2-year follow-up of ranibizumab plus prompt or deferred laser or triamcinolone plus prompt laser for diabetic macular edema. Ophthalmology. 2011;118:609-14.

6. Vujosevic S, Bottega E, Casciano M, Pilotto E, Convento E, Midena E. Microperimetry and fundus autofluorescence in diabetic macular edema: subthreshold micropulse diode laser versus modified Early Treatment Diabetic Retinopathy Study laser photocoagulation. Retina. 2010;30: 908-16.

7. Inagaki K, Ohkoshi K, Ohde S. Spectral domain optical coherence tomography imaging of retinal changes after conventional multicolor laser, subthreshold micropulse diode laser or pattern scanning laser therapy in Japanese with macular edema. Retina. 2012;32:1592-600.

8. Lavinsky D, Cardillo J, Melo L, Dare A, Farah M, Belfort R. Randomized clinical trial evaluating mETDRS versus normal or high-density micropulse photocoagulation for diabetic macular edema. Invest Ophthalmol Vis Sci. 2011;52:4314-23. 


\title{
Antiangiogénicos en el edema macular diabético
}

\author{
René Cano-Hidalgo* \\ Instituto de Oftalmología FAP Conde de Valenciana, Ciudad de México, México
}

El factor de crecimiento endotelial vascular (VEGF, vascular endothelial growth factor) tiene una importancia crucial en la patogénesis del edema macular diabético $(E M D)$, generando principalmente una rotura de la barrera hematorretiniana (BHR), pérdida de la autorregulación de la circulación retiniana, dilatación venosa y cambios histopatológicos, tales como engrosamiento de la membrana basal capilar y apoptosis de las células y los pericitos endoteliales ${ }^{1,2}$.

La fisiopatología compleja de la formación del EMD, expuesta en capítulos previos, no solo está influenciada por las células inflamatorias y gliales, sino también por el aumento de la expresión de moléculas inflamatorias, incluidas las interleucinas, el factor de necrosis tumoral alfa, el factor de crecimiento transformante beta, las metaloproteinasas de la matriz y el VEGF 3 .

En la actualidad, de acuerdo con la evidencia científica, los medicamentos que bloquean la acción del VEGF son el tratamiento de primera línea, ya que restauran la integridad de la BHR, resuelven el edema macular y mejoran la visión en la mayoría de los pacientes con $\mathrm{EMD}^{1,3}$.

Los medicamentos que bloquean el VEGF funcionan principalmente restableciendo la BHR, «normalizando» la circulación y evitando el crecimiento de nuevos vasos sanguíneos. Los ojos humanos sanos tienen concentraciones de VEGF en el vítreo de $8.8 \pm 99 \mathrm{ng} / \mathrm{ml}$, mientras que en los pacientes con EMD y otras afecciones vasculares retinianas las concentraciones intraoculares son elevadas. Se ha evidenciado que después de la vitrectomía vía pars plana la vida media intravítrea del VEGF en ojos de conejo disminuyó un $75 \%{ }^{4}$.
El desarrollo de la terapia anti-VEGF ha sido uno de los avances más importantes en la oftalmología. En la actualidad existen agentes anti-VEGF que en estudios clínicos han demostrado su eficacia en el tratamiento del EMD: bevacizumab, ranibizumab y aflibercept.

En la tabla 1 se describen las principales características de los medicamentos que se unen al VEGF e inhiben su acción, utilizados en el tratamiento de la angiogénesis ocular.

\section{Bevacizumab}

El bevacizumab (Avastin ${ }^{\circledR}$, Genentech Inc., San Francisco, CA, EUA) fue el primer agente antiangiogénico clínicamente disponible en los Estados Unidos de América. Es un anticuerpo anti-VEGF humanizado recombinante activo contra todas las isoformas del VEGF, aprobado por la Food and Drug Administration (FDA) en 2004 como terapia sistémica para el tratamiento del cáncer colorrectal metastásico. Tras la aprobación del bevacizumab para el tratamiento del cáncer y el descubrimiento del papel que tiene en la neovascularización ocular, se evidenció que su uso favorece una mejor función visual en pacientes con degeneración macular relacionada con la edad (AMD, age-related macular degeneration). La región Fc del anticuerpo bevacizumab se une a los receptores FcRn que se expresan altamente en las células endoteliales vasculares ${ }^{5,6}$.

La dosis estándar de bevacizumab intraocular es de $1.25 \mathrm{mg}$ en $0.05 \mathrm{ml}$. La vida media intravítrea del bevacizumab en muestras vítreas humanas después de una sola inyección ha sido reportada entre 6.7 y 9.82 días, 
Tabla 1. Características de los fármacos anti-VEGF

\begin{tabular}{|c|c|c|c|c|}
\hline Característica & Bevacizumab & Ranibizumab & Aflibercept & Brolicizumab \\
\hline Descripción & $\begin{array}{l}\text { Anticuerpo múrido, } \\
\text { recombinante, } \\
\text { humanizado, } \\
\text { contra VEGF-A }\end{array}$ & $\begin{array}{l}\text { Fragmento de anticuerpo } \\
\text { múrido, recombinante, } \\
\text { humanizado, contra } \\
\text { VEGF-A }\end{array}$ & $\begin{array}{l}\text { Proteína de fusión con } \\
\text { secuencias de receptor } \\
\text { unidas al fragmento Fc de IgG }\end{array}$ & $\begin{array}{l}\text { Fragmento de } \\
\text { anticuerpo humanizado } \\
\text { de cadena sencilla }\end{array}$ \\
\hline Peso molecular (kDa) & 149 & 48 & 115 & 26 \\
\hline Unión a Isoformas & VEGF-A & VEGF-A & $\begin{array}{l}\text { VEGF-A, VEGF-B, factor de } \\
\text { crecimiento placentario }\end{array}$ & VEGF-A \\
\hline $\begin{array}{l}\text { Afinidad de unión } \\
\text { por VEGF }_{165} \text { (pmol) }\end{array}$ & $58-1000$ & 50 & 0.5 & \\
\hline $\begin{array}{l}\text { Vida media } \\
\text { intravítrea (días) }\end{array}$ & 9.8 & 7.1 & 9 & \\
\hline $\begin{array}{l}\text { Vida media en } \\
\text { sangre (días) }\end{array}$ & 21 & $2-4$ & 6 & \\
\hline
\end{tabular}

con un promedio de 8.25 días. La retención sistémica después del tratamiento intravítreo con bevacizumab se midió en pacientes con EMD, con concentraciones plasmáticas sistémicas de VEGF significativamente reducidas hasta 4 semanas después de una sola inyección de bevacizumab.

Los viales de bevacizumab se pueden dividir en varias dosis y el costo es mucho más bajo en comparación con el ranibizumab y el aflibercept. Dado que el bevacizumab no está aprobado para el tratamiento de la enfermedad ocular, existe una necesidad imperiosa de contar con sólidos datos comparativos de eficacia y seguridad ${ }^{4,6}$.

\section{Evidencia}

Diversos estudios clínicos han demostrado el beneficio del bevacizumab en el EMD:

- El tratamiento con bevacizumab intravítreo reduce la neovascularización en el iris y la retina; sin embargo, el efecto es corto, ya que se encontró la recurrencia de la neovascularización después de 2 semanas ${ }^{7}$.

- El Protocolo H de DRCR.net fue un estudio piloto a corto plazo que aleatorizó ojos con EMD a dos dosis diferentes de bevacizumab intravítreo $(1.25 \circ 2.5 \mathrm{mg})$ o láser focal. En comparación con el tratamiento solo con láser focal, los ojos tratados con bevacizumab tuvieron una mayor reducción del grosor retiniano central a las 3 semanas y mejoría de la agudeza visual corregida de aproximadamente una línea a las 12 semanas. No se encontró diferencia entre las dosis de 1.25 y $2.5 \mathrm{mg}$ en los efectos a corto plazo. Los datos de este estudio proporcionaron una evidencia temprana limitada de que el tratamiento con bevacizumab intravítreo puede beneficiar a los ojos con EMD ${ }^{5,8}$.

- En el estudio realizado por el Pan-American Collaborative Retina Study Group (PACORES) se revisaron los datos clínicos de 139 ojos con EMD tratados con bevacizumab en 11 centros de ocho países. Los pacientes tratados con bevacizumab intravítreo (1.25 o $2.5 \mathrm{mg}$ ) tuvieron una mejoría significativa de la agudeza visual y del grosor retiniano central al mes de la primera inyección, con resultados mantenidos a los 24 meses. El $51.8 \%$ de los ojos mejoraron en dos o más líneas del ETDRS, el $44.6 \%$ de los ojos permanecieron estables y el $3.6 \%$ disminuyeron en dos o más líneas. A los 24 meses, el grosor retiniano central disminuyó de $446.4 \pm 154.4$ a $279.7 \pm 80 \mu \mathrm{m}$. El número promedio de inyecciones por ojo fue de 5.8 y no hubo diferencias significativas en el efecto entre las dosis de 1.25 y $2.5 \mathrm{mg}^{5,8}$.

- El estudio BOLT fue un estudio prospectivo, aleatorizado, controlado, de bevacizumab intravítreo frente a terapia con láser en el tratamiento del EMD. Los resultados a 2 años mostraron una mejoría de la agudeza visual en el grupo de bevacizumab en comparación con los ojos tratados con láser. Alrededor del $32 \%$ de los pacientes en el grupo de bevacizumab ganaron 15 letras o más, en comparación con el $4 \%$ en el grupo de láser. Este estudio no informó eventos adversos graves durante 12 meses y apoyó el uso de bevacizumab intravítreo para el tratamiento del EMD, principalmente en pacientes con involucro del centro macular sin isquemia macular avanzada. 
Las mejoras en la agudeza visual corregida y en el grosor macular central observadas con bevacizumab al año se mantuvieron durante el segundo año con una media de cuatro inyecciones, lo que proporcionó evidencia que respalda el uso a largo plazo del bevacizumab intravítreo para el EMD persistente ${ }^{5,8}$.

- En marzo de 2015, el DRCR.net publicó los resultados del primer año del estudio del Protocolo T, un ensayo clínico aleatorizado patrocinado por los $\mathrm{Na}$ tional Institutes of Health. El propósito era comparar la eficacia y la seguridad del aflibercept intravítreo, el bevacizumab y el ranibizumab para el tratamiento del EMD con involucro macular central y compromiso visual. Este estudio proporcionó datos sobre la eficacia y la seguridad del bevacizumab, el aflibercept y el ranibizumab. Según sus resultados, la elección del tratamiento para el EMD depende de la puntuación inicial de la agudeza visual mejor corregida. Si bien el aflibercept y el ranibizumab fueron los medicamentos de elección para un puntaje de agudeza visual corregida $<69$, los tres medicamentos fueron equivalentes para mejorar la visión en los ojos con un puntaje $\geq 69$. El número de eventos adversos graves fue pequeño, pero el seguimiento fue corto. El costo mucho menor del bevacizumab intravítreo fue indiscutible ${ }^{6}$.

\section{Ranibizumab}

Los científicos de Genentech reconocieron que la supresión del VEGF podría usarse para tratar la degeneración macular, el EMD y el edema macular debido a oclusiones de la vena retiniana, pero les preocupaba que un anticuerpo de longitud completa como el bevacizumab pudiera tener una eficacia limitada y un perfil de seguridad no favorable, por lo que continuaron realizando investigaciones ${ }^{4,5}$. El ranibizumab (Lucentis ${ }^{\circledR}$, Genentech USA, Inc., San Francisco, CA, EUA/Novartis Ophthalmics, Basilea, Suiza), un anticuerpo de $48 \mathrm{kDa}$, se une a todas las isoformas del VEGF-A y posee una afinidad molar por el $\mathrm{VEGF}_{165}$ de 5 a 20 veces mayor que la del bevacizumab. Como un pequeño fragmento de anticuerpo que carece de un dominio Fc, tiene una vida media más corta que los otros agentes anti-VEGF. El ranibizumab intravítreo fue el segundo agente antiVEGF con licencia para tratar la AMD neovascular ${ }^{4,5}$.

La justificación de la terapia anti-VEGF para el EMD se basó en la observación de que los valores de VEGF aumentan en la retina y en el vítreo de los ojos con retinopatía diabética. Después de completar con éxito los ensayos de fase III, el ranibizumab fue aprobado por la FDA en 2006 para el tratamiento de la AMD neovascular. En ojos humanos, la vida media intravítrea varió de 7.1 a 9 días. Se ha observado que el ranibizumab causa una mayor reducción en la proliferación de células endoteliales, mientras que el bevacizumab tiene un mayor efecto sobre la migración, la formación de tubos y la fosforilación del VEGFR-24,6.

Los estudios farmacocinéticos mostraron que las concentraciones de ranibizumab clínicamente alcanzables dentro de la retina con inyecciones intravítreas fueron capaces de suprimir la actividad angiogénica durante 1 mes. Se ha demostrado que la inyección intravítrea de ranibizumab es benéfica y relativamente segura para el tratamiento del EMD, por lo que se ha convertido en un estándar para su tratamiento ${ }^{4,6}$.

\section{Evidencia}

Diversos estudios clínicos han demostrado el beneficio del ranibizumab en el EMD. Los estudios READ-2, RIDE y RISE, RESOLVE, RESTORE y PROTOCOLO I demostraron la superioridad del ranibizumab en los resultados de agudeza visual y disminución del grosor macular en comparación con el tratamiento con láser macular ${ }^{4}, 6$ :

- READ-2 FASE II (2006): primer estudio prospectivo, aleatorizado, doble ciego y multicéntrico de ranibizumab para el EMD. Se comparó el ranibizumab con el láser focal/rejilla y la combinación de ambos en el EMD. A los 6 meses, las inyecciones de ranibizumab tuvieron un mejor resultado visual que el láser focal. Así mismo, el ranibizumab proporcionó un beneficio en el tratamiento del EMD al menos de 2 años, y en combinación con el láser (focal/rejilla) se redujeron la cantidad de edema residual y la frecuencia de inyecciones requeridas. El estudio demostró que el ranibizumab intravítreo, con o sin fotocoagulación macular con láser, fue superior al láser para el tratamiento del EMD y tuvo un perfil de seguridad aceptable ${ }^{4,8}$.

- RIDE y RISE (2007): estudio de ranibizumab en pacientes con edema macular clínicamente significativo con afectación central secundario a retinopatía diabética. Se compararon la eficacia y la seguridad de 0.3 y $0.5 \mathrm{mg}$ de ranibizumab frente al tratamiento simulado o láser en pacientes con EMD con involucro central. Los ensayos a 3 años asignaron al azar a 759 pacientes a tres grupos de tratamiento: $0.3 \mathrm{mg}$ de ranibizumab mensual, $0.5 \mathrm{mg}$ de ranibizumab mensual o tratamiento simulado. Se encontró que el ranibizumab mejoró la agudeza visual de manera rápida y 
mantenida, redujo el riesgo de una mayor pérdida de visión y mejoró el edema macular en los pacientes con EMD, con bajas tasas de daño ocular y no ocular ${ }^{4,8}$.

- PROTOCOLO I, DRCR.net: este estudio proporcionó evidencia de primer nivel que apoyaba el uso de ranibizumab en ojos con EMD. El protocolo I fue un estudio multicéntrico de 5 años que aleatorizó 854 ojos con EMD con involucro del centro foveal para recibir una inyección placebo y láser inmediato, $0.5 \mathrm{mg}$ de ranibizumab y láser inmediato, $0.5 \mathrm{mg}$ de ranibizumab y láser diferido (24 semanas) o triamcinolona y láser inmediato. Los resultados mostraron que el ranibizumab intravítreo con láser inmediato o diferido fue más efectivo a los 2 años en comparación con el láser inmediato solo para el tratamiento del EMD con involucro macular central. En los ojos pseudofáquicos, la triamcinolona intravítrea con láser rápido pareció ser más efectiva que el láser solo, pero con un aumento del riesgo de elevación de la presión intraocular ${ }^{8}$.

- RESOLVE fase II: en este estudio a 12 meses se evaluaron la seguridad y la eficacia del ranibizumab en el EMD con afectación central. Se aleatorizó a 152 pacientes para recibir mensualmente $0.3 \mathrm{mg}$ de ranibizumab, $0.5 \mathrm{mg}$ de ranibizumab o inyecciones simuladas. La agudeza visual corregida mejoró en +10.3 letras en los grupos de ranibizumab, pero disminuyó en -1 letra en el grupo de tratamiento simulado. La mejoría promedio de la disminución del grosor retiniano central fue de $-194 \mu \mathrm{m}$ para los grupos de ranibizumab y $-48 \mu \mathrm{m}$ para el grupo de tratamiento simulado. Este estudio aportó evidencia de que el ranibizumab es efectivo para mejorar la agudeza visual y bien tolerado ${ }^{4,8}$.

- RESTORE fase III (2008): este estudio clínico de fase III, multicéntrico (75 sitios), llevado a cabo en el hemisferio oriental, incluyó 345 pacientes que fueron asignados al azar para recibir ranibizumab y láser simulado, ranibizumab y láser, o inyecciones simuladas y láser. El objetivo principal era demostrar la superioridad de la monoterapia con ranibizumab o de la monoterapia con ranibizumab y láser sobre la monoterapia con láser, medida por la mejoría de la visión a los 12 meses. Se encontró que la monoterapia con ranibizumab y la combinación con láser proporcionaron una ganancia de agudeza visual superior a la lograda con el láser estándar en pacientes con compromiso visual debido a EMD. Las ganancias de agudeza visual se asociaron con ganancias significativas en las puntuaciones de VFQ-25. La monoterapia con ranibizumab y la combinación con láser presentaron un perfil de seguridad en el EMD similar al observado en la $\mathrm{AMD}^{4,8}$.

\section{Aflibercept}

El aflibercept $\left(\right.$ Eylea $^{\circledR}$, Regeneron, Tarrytown, Nueva York, EUA), aprobado por la FDA en 2011, es una proteína de fusión que comprende el segundo dominio de inmunoglobulina del receptor 1 del VEGF, el tercer dominio de inmunoglobulina del receptor 2 del VEGF y la porción Fc de la IgG1 humana. Tiene 100 veces más afinidad de unión que el ranibizumab y el bevacizumab. Además, su vida media intravítrea es de 4.8 días, en comparación con 3.2 días para el ranibizumab y 5.6 días para el bevacizumab. Se cree que estas características del aflibercept permiten menos inyecciones, con una eficacia similar en comparación con los otros agentes anti-VEGF. En julio de 2014 la FDA aprobó el aflibercept para el tratamiento del EMD. Además de inhibir el VEGF, también bloquea la acción del factor de crecimiento placentario, por lo que ha demostrado su eficacia y seguridad en ensayos clínicos en el tratamiento del EMD ${ }^{5,6}$.

\section{Evidencia}

Diversos estudios clínicos han demostrado el beneficio del aflibercept en el EMD:

- Estudio DA VINCI (2008): estudio prospectivo, aleatorizado y controlado clave que permitió demostrar la eficacia del aflibercept para el tratamiento del EMD. Se aleatorizaron 221 pacientes con EMD y compromiso foveal central $\geq 250 \mu \mathrm{m}$ para recibir aflibercept intravítreo en diferentes dosis y horarios (cuatro grupos: $0.5 \mathrm{mg}$ cada 4 semanas, $2 \mathrm{mg}$ cada 4 semanas, $2 \mathrm{mg}$ cada 8 semanas después de 3 inyecciones de carga mensual, y $2 \mathrm{mg}$ por razón necesaria después de 3 dosis de carga mensual) o tratamiento con láser. Los grupos que recibieron aflibercept tuvieron un cambio promedio en la agudeza visual corregida de 8.5 a 11.4 letras, en comparación con 2.5 letras en el grupo con láser, en la semana 24. El grosor retiniano central también disminuyó significativamente después de la administración de aflibercept en comparación con el grupo que recibió láser. No se observaron diferencias significativas entre los grupos de aflibercept, lo que respalda el régimen de dosificación cada 8 semanas. Al primer año, el cambio en el promedio de agudeza visual corregida varió de 9.7 a 13.1 letras ganadas en los grupos de aflibercept, frente a una pérdida de 1.3 letras en el grupo con láser ${ }^{4,5}$. 
- VIVID y VISTA: estos estudios de fase III compararon la eficacia y la seguridad de dos regímenes de dosificación de aflibercept con láser macular para el EMD y fueron vitales para la aprobación de este fármaco por la FDA. Por sus resultados, el aflibercept fue aprobado en Europa (2014) por la Agencia Europea de Medicamentos y en los Estados Unidos de América (2015) por la FDA para el tratamiento del EMD. La dosis aprobada fue de $2 \mathrm{mg}$ por inyección. El fármaco fue aprobado para cinco inyecciones mensuales seguidas de inyecciones bimensuales en los Estados Unidos de América, y la etiqueta europea agrega la posibilidad de extender el intervalo después del primer año de tratamiento ${ }^{6}$.

- Protocol T, DRCR.net: estudio multicéntrico, aleatorizado y controlado que comparó el aflibercept, el ranibizumab y el bevacizumab para el tratamiento del EMD. Se aleatorizaron 660 pacientes de 89 centros para recibir $1.25 \mathrm{mg}$ de bevacizumab, $0.3 \mathrm{mg}$ de ranibizumab o $2 \mathrm{mg}$ de aflibercept. Los criterios de inclusión fueron tener una agudeza visual corregida de 20/32 a 20/320 y EMD con involucro del centro por examen clínico y de tomografía de coherencia óptica 4 .

\section{Brolucizumab}

El brolucizumab, formalmente RTH258, es un fragmento de anticuerpo humanizado de cadena sencilla que inhibe todas las isoformas del VEGF-A. Fue desarrollado por ESBA Tech (descubrimiento en la fase 2a), Alcon Laboratories (fase 2b) y Novartis (fase 3). Tiene un peso molecular pequeño $(26 \mathrm{kDa})$, siendo el anticuerpo monoclonal más pequeño creado, lo que permite que penetre en los tejidos y se elimine más rápido de la circulación sistémica en comparación con las moléculas más grandes.

Los estudios HAWK y HARRIER fueron diseñados para evaluar la eficacia y la seguridad del brolucizumab en comparación con el aflibercept en pacientes con degeneración macular húmeda, y permitieron la aprobación del brolucizumab por la FDA en 2019 para el tratamiento de la forma exudativa en pacientes con $\mathrm{AMD}^{9-12}$.

El brolucizumab se une e inhibe las tres isoformas principales del VEGF-A $\left(\mathrm{VEGF}_{110}, \mathrm{VEGF}_{121}\right.$ y $\left.\mathrm{VEGF}_{165}\right)$, y previene la interacción con los receptores VEGFR-1 y VEGFR-2, suprimiendo la proliferación celular endotelial, la neovascularización y la permeabilidad vascular. Además, se ha descrito una afinidad por el VEGF-A humano similar a la del ranibizumab y mayor que la del bevacizumab. Su farmacocinética intraocular se investigó in vivo en monos Cynomolgus y se encontró que después de una inyección intravítrea bilateral única de brolucizumab (1.0 o $6.0 \mathrm{mg}$ por ojo) se produjo un aclaramiento paralelo de todos los compartimentos oculares, con una vida media terminal de 56.8 horas $^{12}$.

El régimen de dosificación recomendado es de $6 \mathrm{mg}$ ( $0.05 \mathrm{ml}$ de una solución de $120 \mathrm{mg} / \mathrm{ml}$ ) en inyección intravítrea mensual las primeras tres dosis, y luego una inyección cada 8-12 semanas ${ }^{12}$.

En cuanto a los resultados de los estudios HAWK (NCT02307682) y HARRIER (NCT02434328), el brolucizumab se asoció con ganancias en la agudeza visual y mostró no inferioridad en comparación con el aflibercept en pacientes con neovascularización coroidea activa no tratada asociada a AMD. En estos ensayos clínicos se evaluaron diferentes dosis: en el estudio HAWK 3 y $6 \mathrm{mg}$ de brolucizumab, y en el estudio HARRIER solo $6 \mathrm{mg}^{13}$.

\section{Evidencia}

Existen varios estudios de fase III que comparan el brolucizumab y el aflibercept en pacientes con EMD, los cuales se encuentran en fases iniciales o reclutando pacientes, como KESTREL, KITE, BUZZARD, KINGLET y KING-FISHER; sin embargo, en la actualidad no se cuenta con datos robustos de su eficacia en el mundo real ${ }^{12}$.

\section{Bibliografía}

1. Urbancic M, Klobucar P, Zupan M, Urbancic K, Lavric A. Anti-VEGF treatment of diabetic macular edema: two-year visual outcomes in routine clinical practice. J Ophthalmol. 2020;2020:6979758.

2. Sharma A, Bellala K, Dongre P, Reddy P. Anti-VEGF versus dexamethasone implant for the management of Centre involved Diabetic Macular Edema (CiDME): a randomized study. Int Ophthalmol. 2020;40:67-72.

3. Kwon J, Jee D. Aqueous humor cytokine levels in patients with diabetic macular edema refractory to anti-VEGF treatment. PLoS One. 2018;13:e0203408.

4. Stewart M. Diabetic retinopathy: current pharmacologic treatment and emerging strategies. Singapore: Springer Verlag; 2017. 268 p.

5. Klein K, Liang M. Anti-VEGF therapy for diabetic eye disease. En: Baumal CR, Duker J, editores. Current management of diabetic retinopathy. Philadelphia: Elsevier; 2018. p. 53-78.

6. Schmidt U, Garcia J, Bandello F, Berg K, Chakravarthy U, Gerendas B et al. Guidelines for the management of diabetic macular edema: European Society of Retina Specialists (EURETINA). Ophthalmologica. 2017; $237: 185-222$

7. Lu L, Jiang $Y$, Jaganathan R, Hao Y. Review article. Current advances in pharmacotherapy and technology for diabetic retinopathy: a systematic review. J Ophthalmol. 2018;2018;1694187.

8. Gupta A, Aggarwal V, Goel N. Handbook of clinical trials in ophthalmology (PDF). New Delhi, India: Jaypee Brothers Medical Publishers; 2014. 336 p.

9. Sharma A, Parachuri N, Sharma R, Bandello F, Kuppermann B, Loewenstein A. Brolucizumab - another anti-VEGF or beyond. Eye (Lond). 2020;34:1499-500

10. Grzybowski A. Current concepts in ophthalmology. Springer Nature Switzerland; 2020. $300 \mathrm{p}$.

11. Sharma A, Kumar N, Parachuri N, Sharma R, Bandello F, Kuppermann B, et al. Brolucizumab and immunogenicity. Eye (Lond). 2020;34:1726-8.

12. Markham A. Brolucizumab: first approval. Drugs. 2019;79:1997-2000.

13. Sharma A, Parachuri N, Kumar N, Sharma R, Bandello F, Kuppermann B, et al. Brolucizumab-key learnings from HAWK and HARRIER. Eye (Lond). 2020;34:1318-20 


\section{Estudios multicéntricos actualizados para el tratamiento del edema macular diabético}

\section{Enrique Roig-Melo*}

Instituto de Investigación y Ciencias Visuales, Universidad de Guadalajara, Guadalajara, Jalisco, México

\begin{tabular}{|c|c|c|c|c|}
\hline & Tipo de estudio & Objetivo & Resultado & Conclusiones \\
\hline $\begin{array}{l}\text { Estudio } \\
\text { RESOLVE } \\
\text { Massin, et al. }{ }^{1} \\
2010\end{array}$ & $\begin{array}{l}\text { Estudio de fase 2, } \\
\text { multicéntrico, } \\
\text { aleatorizado, doble } \\
\text { ciego, con duración } \\
\text { de } 12 \text { meses }\end{array}$ & $\begin{array}{l}\text { Evaluar la eficacia y la } \\
\text { seguridad del } \\
\text { ranibizumab en el } \\
\text { EMD con involucro } \\
\text { foveal }\end{array}$ & $\begin{array}{l}\text { El promedio de cambio en } \\
\text { AVMC desde la basal y } \\
\text { hasta el mes } 12 \text { fue } \\
\text { estadísticamente superior } \\
\text { con ranibizumab ( } 7.8 \text { letras) } \\
\text { que con placebo (-0-1 } \\
\text { letras) }\end{array}$ & $\begin{array}{l}\text { Se obtuvieron mejores resultados } \\
\text { en AVMC y GCR con ranibizumab } \\
\text { en comparación con placebo }\end{array}$ \\
\hline $\begin{array}{l}\text { Estudio } \\
\text { RESTORE } \\
\text { Mitchell, et al. }{ }^{2} \text {, } \\
2011\end{array}$ & $\begin{array}{l}\text { Estudio } \\
\text { multicéntrico, doble } \\
\text { ciego, con duración } \\
\text { de } 12 \text { meses, en } 73 \\
\text { centros de Europa, } \\
\text { Turquía, Canadá y } \\
\text { Australia }\end{array}$ & $\begin{array}{l}\text { Demostrar la } \\
\text { superioridad del } \\
\text { ranibizumab } 0.5 \mathrm{mg} \\
\text { como monoterapia o } \\
\text { combinado con láser } \\
\text { frente al uso de láser } \\
\text { solo en el cambio } \\
\text { promedio en AVMC a } \\
\text { los } 12 \text { meses en } \\
\text { pacientes con EMD }\end{array}$ & $\begin{array}{l}\text { Al mes } 12 \text {, un mayor } \\
\text { porcentaje de pacientes } \\
\text { presentaron } A V M C \geq 15 \\
\text { con ranibizumab }(53 \%) \text { en } \\
\text { comparación con los que } \\
\text { solo recibieron láser } \\
(23.6 \%)\end{array}$ & $\begin{array}{l}\text { El ranibizumab en monoterapia o } \\
\text { combinado con láser es superior } \\
\text { al láser solo (rápida mejoría en AV } \\
\text { y mantenida) en pacientes con } \\
\text { EMD } \\
\text { El ranibizumab mostró un } \\
\text { porcentaje mayor de pacientes } \\
\text { con GCR } \leq 275 \mu \mathrm{m} \text { en comparación } \\
\text { con el láser }\end{array}$ \\
\hline $\begin{array}{l}\text { Estudio } \\
\text { RESTORE } \\
\text { (extensión) } \\
\text { Schmidt, et al. }{ }^{3} \text {, } \\
2014\end{array}$ & $\begin{array}{l}\text { Estudio de fase } 3 b \text {, } \\
\text { multicéntrico, de } \\
\text { extensión a } 24 \\
\text { meses }\end{array}$ & $\begin{array}{l}\text { Evaluar la eficacia y la } \\
\text { seguridad a largo } \\
\text { plazo ( } 3 \text { años) del } \\
\text { tratamiento } \\
\text { individualizado con } \\
\text { ranibizumab en } \\
\text { pacientes con daño } \\
\text { visual debido a EMD }\end{array}$ & $\begin{array}{l}\text { Durante el periodo de } \\
\text { extensión del estudio, los } \\
\text { pacientes tratados con } \\
\text { ranibizumab mantuvieron la } \\
\text { AVMC y el grosor retinal } \\
\text { central observados al mes } \\
12\end{array}$ & $\begin{array}{l}\text { Al mes } 36 \text {, los pacientes tratados } \\
\text { con láser recibieron ranibizumab y } \\
\text { alcanzaron una AVMC similar a la } \\
\text { del grupo de ranibizumab inicial, } \\
\text { mantenida a lo largo del tiempo } \\
\text { El ranibizumab demostró un perfil } \\
\text { favorable de eficacia y seguridad } \\
\text { a largo plazo }\end{array}$ \\
\hline $\begin{array}{l}\text { Estudio READ-2 } \\
\text { Nguyen, et al. }{ }^{4} \\
2009\end{array}$ & $\begin{array}{l}\text { Estudio de fase 2, } \\
\text { aleatorizado, } \\
\text { multicéntrico (14 } \\
\text { centros de los } \\
\text { Estados Unidos de } \\
\text { América) }\end{array}$ & $\begin{array}{l}\text { Comparar ranibizumab } \\
\text { con FTC en rejilla o la } \\
\text { combinación de } \\
\text { ambos para el } \\
\text { tratamiento del EMD }\end{array}$ & $\begin{array}{l}\text { Al mes } 6 \text {, la ganancia } \\
\text { promedio en AVMC fue } \\
\text { significativamente mayor } \\
\text { en el grupo de ranibizumab } \\
\text { que en el grupo con FTC } \\
\text { en rejilla }\end{array}$ & $\begin{array}{l}\text { Los pacientes que recibieron } \\
\text { ranibizumab en cualquiera de los } \\
\text { grupos tuvieron una mayor } \\
\text { ganancia de letras a los } 6 \text { meses } \\
\text { que aquellos que recibieron láser } \\
\text { en monoterapia }\end{array}$ \\
\hline
\end{tabular}




\begin{tabular}{|c|c|c|c|c|}
\hline & Tipo de estudio & Objetivo & Resultado & Conclusiones \\
\hline $\begin{array}{l}\text { Estudio READ-2 } \\
\text { ( } 2 \text { años) } \\
\text { Nguyen, et al. }{ }^{5} \\
2010\end{array}$ & $\begin{array}{l}\text { Estudio de fase 2, } \\
\text { aleatorizado, } \\
\text { multicéntrico ( } 14 \\
\text { centros de los } \\
\text { Estados Unidos de } \\
\text { América) }\end{array}$ & $\begin{array}{l}\text { Determinar los efectos } \\
\text { a largo plazo del } \\
\text { ranibizumab en } \\
\text { pacientes con EMD }\end{array}$ & $\begin{array}{l}\text { Después de obtener el } \\
\text { resultado principal a } 6 \\
\text { meses, la mayoría de los } \\
\text { pacientes recibieron } \\
\text { tratamiento solo con } \\
\text { ranibizumab, un número } \\
\text { promedio de inyecciones } \\
\text { de } 5.3,4.4 \text { y } 2.9 \text { durante el } \\
\text { periodo de seguimiento de } \\
18 \text { meses, y en estos } \\
\text { grupos la mejoría promedio } \\
\text { de la AVMC fue de } 7.4,0.5 \\
\text { y } 3.8 \text { letras, } \\
\text { respectivamente }\end{array}$ & $\begin{array}{l}\text { El ranibizumab brinda buenos } \\
\text { resultados funcionales, pero el } \\
\text { esquema de tratamiento debe } \\
\text { personalizarse }\end{array}$ \\
\hline $\begin{array}{l}\text { Estudio READ-2 } \\
\text { (3 años) } \\
\text { Do, et al. }{ }^{6} \\
2013\end{array}$ & $\begin{array}{l}\text { Estudio de fase 2, } \\
\text { aleatorizado, } \\
\text { multicéntrico } \\
\text { (llevado a cabo en } \\
14 \text { centros de los } \\
\text { Estados Unidos de } \\
\text { América) }\end{array}$ & $\begin{array}{l}\text { Evaluar el beneficio de } \\
\text { extender el } \\
\text { seguimiento y el } \\
\text { tratamiento con } \\
\text { ranibizumab entre los } \\
\text { meses } 24 \text { y } 36 \text { en } \\
\text { pacientes con EMD } \\
\text { del estudio READ- } 2\end{array}$ & $\begin{array}{l}\text { La mejoría promedio desde } \\
\text { la basal en la AVMC en el } \\
\text { grupo de ranibizumab fue } \\
\text { de } 10.3 \text { letras al mes } 36, \\
\text { frente a } 7.2 \text { letras al mes } \\
24\end{array}$ & $\begin{array}{l}\text { Existe una gran heterogeneidad } \\
\text { entre los pacientes con EMD, por } \\
\text { lo que es recomendable } \\
\text { individualizar el tratamiento; } \\
\text { algunos pacientes requieren de } \\
\text { dosis más frecuentes }\end{array}$ \\
\hline $\begin{array}{l}\text { Estudio READ-3 } \\
\text { ( } 6 \text { meses) } \\
\text { Do, et al. } \\
2015\end{array}$ & $\begin{array}{l}\text { Estudio } \\
\text { aleatorizado, doble } \\
\text { ciego, multicéntrico } \\
\text { (llevado a cabo en } \\
13 \text { centros de los } \\
\text { Estados Unidos de } \\
\text { América) }\end{array}$ & $\begin{array}{l}\text { Comparar inyecciones } \\
\text { de ranibizumab de } 2 \mathrm{y} \\
0.5 \mathrm{mg} \text { en ojos con } \\
\text { EMD con involucro } \\
\text { central por TD-OCT } \geq \\
250 \mu \mathrm{m}\end{array}$ & $\begin{array}{l}\text { Al mes } 6 \text {, la mejoría } \\
\text { promedio en el grupo que } \\
\text { recibió } 0.5 \mathrm{mg} \text { fue de }+9.43 \\
\text { letras, y de }+7.01 \text { letras en } \\
\text { el grupo que recibió } 2 \mathrm{mg}\end{array}$ & $\begin{array}{l}\text { No hubo diferencia } \\
\text { estadísticamente significativa } \\
\text { entre la AVMC final entre ambas } \\
\text { dosis } \\
\text { La dosis de } 2 \mathrm{mg} \text { de ranibizumab } \\
\text { no es superior en el resultado final } \\
\text { a la dosis de } 0.5 \mathrm{mg} \text { para el } \\
\text { tratamiento del EMD }\end{array}$ \\
\hline $\begin{array}{l}\text { Estudio READ-3 } \\
\text { ( } 24 \text { meses) } \\
\text { Sepah, et al. }{ }^{8} \\
2016\end{array}$ & $\begin{array}{l}\text { Estudio } \\
\text { aleatorizado, doble } \\
\text { ciego, multicéntrico } \\
\text { (Ilevado a cabo en } \\
13 \text { centros de los } \\
\text { Estados Unidos de } \\
\text { América) }\end{array}$ & $\begin{array}{l}\text { Comparar las dosis de } \\
2 \text { y } 0.5 \mathrm{mg} \text { de } \\
\text { ranibizumab en ojos } \\
\text { con EMD con } \\
\text { involucro central a } 24 \\
\text { meses }\end{array}$ & $\begin{array}{l}\text { Al mes } 24 \text {, la mejoría } \\
\text { promedio desde la basal } \\
\text { en la AVMC fue de }+11.6 \\
\text { letras en el grupo que } \\
\text { recibió } 0.5 \mathrm{mg} \text { y de }+6.7 \\
\text { letras en el grupo que } \\
\text { recibió } 2 \mathrm{mg}\end{array}$ & $\begin{array}{l}\text { Desde el mes } 6 \text { y hasta el mes } 24 \text {, } \\
\text { la AVMC se mantuvo para el grupo } \\
\text { de ranibizumab } 2 \mathrm{mg} \text { y mejoró } 2 \\
\text { letras en el grupo de ranibizumab } \\
0.5 \mathrm{mg} \\
\text { Los resultados visuales y } \\
\text { anatómicos a } 6 \text { meses se pueden } \\
\text { mantener hasta por } 2 \text { años } \\
\text { siguiendo un esquema PRN con } \\
\text { criterios de retratamiento estrictos } \\
\text { y seguimiento frecuente }\end{array}$ \\
\hline $\begin{array}{l}\text { Estudio BOLT } \\
\text { Michaelides, } \\
\text { et al. }{ }^{9} \\
2010\end{array}$ & $\begin{array}{l}\text { Estudio de fase } 3, \\
\text { aleatorizado, } \\
\text { prospectivo, doble } \\
\text { ciego, unicéntrico }\end{array}$ & $\begin{array}{l}\text { Comparar la eficacia } \\
\text { de dosis repetidas de } \\
\text { bevacizumab frente a } \\
\text { la terapia con láser } \\
\text { convencional en } \\
\text { pacientes con EMD }\end{array}$ & $\begin{array}{l}\text { En el grupo que recibió } \\
\text { bevacizumab hubo una } \\
\text { ganancia promedio de } 8 \\
\text { letras ETDRS, mientras que } \\
\text { en el grupo que recibió } \\
\text { tratamiento con láser hubo } \\
\text { una pérdida promedio de } \\
0.5 \text { letras ETDRS }\end{array}$ & $\begin{array}{l}\text { La evidencia respalda el uso del } \\
\text { bevacizumab en pacientes con } \\
\text { involucro central de EMCS sin } \\
\text { isquemia macular avanzada }\end{array}$ \\
\hline $\begin{array}{l}\text { Estudio BOLT } \\
\text { ( } 2 \text { años) } \\
\text { Rajendram, }^{10} \\
\text { et al. }^{10} \text {, } \\
2012\end{array}$ & $\begin{array}{l}\text { Estudio de fase } 3, \\
\text { aleatorizado, } \\
\text { prospectivo, doble } \\
\text { ciego, unicéntrico }\end{array}$ & $\begin{array}{l}\text { Reportar los } \\
\text { resultados a } 2 \text { años } \\
\text { del estudio BOLT }\end{array}$ & $\begin{array}{l}\text { La agudeza visual } \\
\text { promedio a } 2 \text { años fue de } \\
64.4 \text { con bevacizumab y de } \\
54.8 \text { con láser } \\
\text { Con bevacizumab se } \\
\text { obtuvo una ganancia } \\
\text { promedio de } 9 \text { letras, } \\
\text { frente a } 2.5 \text { letras en el } \\
\text { grupo con láser }\end{array}$ & $\begin{array}{l}\text { El bevacizumab es más eficaz que } \\
\text { el láser en pacientes con EMCS }\end{array}$ \\
\hline
\end{tabular}




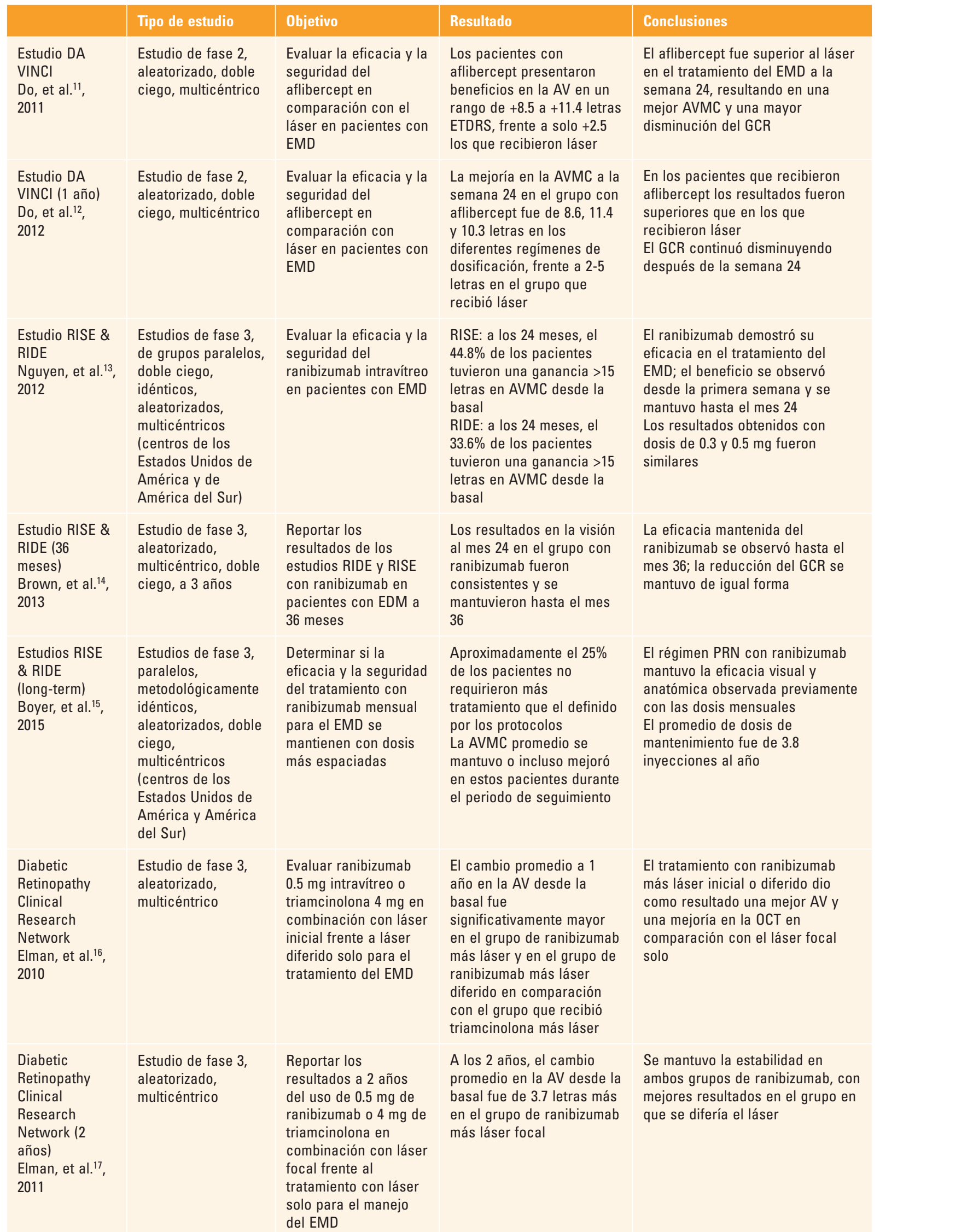




\begin{tabular}{|c|c|c|c|c|}
\hline & Tipo de estudio & Objetivo & Resultado & Conclusiones \\
\hline $\begin{array}{l}\text { Diabetic } \\
\text { Retinopathy } \\
\text { Clinical } \\
\text { Research } \\
\text { Network (5 } \\
\text { años) } \\
\text { Elman, et al. }{ }^{18} \text {, } \\
2015\end{array}$ & $\begin{array}{l}\text { Estudio aleatorizado } \\
\text { y multicéntrico }\end{array}$ & $\begin{array}{l}\text { Reportar los } \\
\text { resultados a } 5 \text { años de } \\
\text { un estudio ya } \\
\text { reportado en el que se } \\
\text { evaluó el uso de } \\
\text { ranibizumab intravítreo } \\
0.5 \text { mg con láser focal } \\
\text { o diferido (por más de } \\
24 \text { semanas) en el } \\
\text { tratamiento del EMD }\end{array}$ & $\begin{array}{l}\text { El cambio promedio en la } \\
\text { AV desde la basal durante } \\
\text { los } 5 \text { años fue de }+7.2 \\
\text { letras en el grupo de láser } \\
\text { focal, frente a }+9.8 \text { letras } \\
\text { en el grupo de láser } \\
\text { diferido }\end{array}$ & $\begin{array}{l}\text { Los resultados a } 5 \text { años indican } \\
\text { que el tratamiento con láser focal } \\
\text { al inicio con la terapia anti-VEGF } \\
\text { no es mejor que el tratamiento con } \\
\text { láser diferido por más de } 24 \\
\text { semanas en ojos con EMD e } \\
\text { involucro central }\end{array}$ \\
\hline $\begin{array}{l}\text { Diabetic } \\
\text { Retinopathy } \\
\text { Clinical } \\
\text { Research } \\
\text { Network (5 } \\
\text { años) } \\
\text { Bressler, } \\
\text { et al. }{ }^{19} \text {, } \\
2016\end{array}$ & Estudio aleatorizado & $\begin{array}{l}\text { Comparar los efectos } \\
\text { anatómicos y la visión } \\
\text { a largo plazo con } \\
\text { ranibizumab con láser } \\
\text { focal o diferido, o } \\
\text { triamcinolona más } \\
\text { láser con ranibizumab } \\
\text { diferido, en pacientes } \\
\text { con EMD }\end{array}$ & $\begin{array}{l}\text { El cambio promedio a } 5 \\
\text { años en ETDRS en los } \\
\text { puntajes de AV desde la } \\
\text { basal en los grupos de } \\
\text { ranibizumab más láser } \\
\text { diferido, ranibizumab más } \\
\text { láser focal, láser más } \\
\text { ranibizumab diferido y } \\
\text { triamcinolona más láser } \\
\text { más ranibizumab diferido, } \\
\text { fue de } 10 \pm 13,8 \pm 13,5 \pm \\
14 \text { y } 7 \pm 14 \text {, } \\
\text { respectivamente }\end{array}$ & $\begin{array}{l}\text { El } 60 \% \text { de los ojos tratados con } \\
\text { láser o triamcinolona más láser } \\
\text { terminaron siendo tratados con } \\
\text { ranibizumab; sin embargo, a } 5 \\
\text { años, en pacientes fáquicos o } \\
\text { pseudofáquicos, los resultados no } \\
\text { fueron similares en comparación } \\
\text { con los pacientes tratados con } \\
\text { ranibizumab desde el inicio }\end{array}$ \\
\hline $\begin{array}{l}\text { DRCR.net } \\
\text { Wells, et al. }{ }^{20} \\
2015\end{array}$ & $\begin{array}{l}\text { Estudio } \\
\text { aleatorizado, } \\
\text { multicéntrico, en } 89 \\
\text { clínicas de los } \\
\text { Estados Unidos de } \\
\text { América }\end{array}$ & $\begin{array}{l}\text { Comparar la eficacia y } \\
\text { la seguridad del uso } \\
\text { intravítreo de } \\
\text { aflibercept, } \\
\text { bevacizumab y } \\
\text { ranibizumab como } \\
\text { tratamiento para el } \\
\text { EMD }\end{array}$ & $\begin{array}{l}\text { Desde la basal y hasta el } \\
\text { primer año, el puntaje en } \\
\text { AV mejoró } 13.3 \text { con } \\
\text { aflibercept, } 9.7 \text { con } \\
\text { bevacizumab y } 11.2 \text { con } \\
\text { ranibizumab }\end{array}$ & $\begin{array}{l}\text { El aflibercept, el bevacizumab y el } \\
\text { ranibizumab intravítreos han } \\
\text { demostrado mejorar la visión en } \\
\text { ojos con EMD con involucro } \\
\text { central; el efecto relativo depende } \\
\text { de la AV basal }\end{array}$ \\
\hline $\begin{array}{l}\text { DRCR.net } \\
\text { (2 años) } \\
\text { Wells, et al. }{ }^{21} \\
2016\end{array}$ & $\begin{array}{l}\text { Estudio } \\
\text { aleatorizado, } \\
\text { multicéntrico, en } 89 \\
\text { clínicas de los } \\
\text { Estados Unidos de } \\
\text { América }\end{array}$ & $\begin{array}{l}\text { Resultados a } 2 \text { años } \\
\text { comparando los } \\
\text { anti-VEGF para el EMD } \\
\text { con seguimiento } \\
\text { estandarizado y } \\
\text { régimen de } \\
\text { tratamiento }\end{array}$ & $\begin{array}{l}\text { El número promedio de } \\
\text { inyecciones de aflibercept, } \\
\text { bevacizumab y ranibizumab } \\
\text { a los } 2 \text { años fue de } 5,6 \text { y } 6 \text {, } \\
\text { respectivamente, y después } \\
\text { de } 2 \text { años fue de } 15,16 \text { y } 15 \text {, } \\
\text { respectivamente } \\
\text { Desde la basal y hasta los } 2 \\
\text { años, la mejoría promedio } \\
\text { en la AV fue de } 12.8 \text { con } \\
\text { aflibercept, } 10 \text { con } \\
\text { bevacizumab y } 12.3 \text { con } \\
\text { ranibizumab }\end{array}$ & $\begin{array}{l}\text { El aflibercept continúa } \\
\text { demostrando superioridad frente } \\
\text { al bevacizumab }\end{array}$ \\
\hline
\end{tabular}

AV: agudeza visual; AVMC: agudeza visual mejor corregida; EMCS: edema macular clínicamente significativo; EMD: edema macular diabético; ETDRS: Early Treatment Diabetic Retinopathy Study; FTC: fotocoagulación; GCR: grosor central de la retina; OCT: tomografía de coherencia óptica; PRN: por razón necesaria; TD-OCT: tomografía de coherencia óptica de dominio de tiempo; VEGF: vascular endothelial growth factor.

\section{Bibliografía}

1. Massin P, Bandello F, Garweg J, Hansen L, Harding S, Larsen M, et al. Safety and efficacy of ranibizumab in diabetic macular edema (RESOLVE Study). Diabetes Care. 2010;33:2399-405.

2. Mitchell P, Bandello F, Schmidt U, Lang G, Massin P, Schlingemann R, et al. Ranibizumab monotherapy of combined with laser versus laser monotheraphy for diabetic macular edema (the RESTORE study). Ophthalmology. 2011;118:615-25.

3. Schmidt U, Lang G, Holz F, Schlingemann R, Lanzetta P, Massin P, et al. Three-year outcomes of individualized ranibizumab treatment in patients with diabetic macular edema. The RESTORE extension study. Ophthalmology. 2014;121:1045-53.

4. Nguyen Q, Mahmood S, Heier J, Abraham P, Campochiaro P. Primary endpoint (six months) of the Ranibizumab for Edema of the mAcula in Diabetes (READ-2 study). Ophthalmology. 2009;116:2175-81
5. Nguyen Q, Mahmood S, Khwaja A, Eliot D, Campochiaro P. Two-year outcomes of the Ranibizumab for Edema of the mAcula in Diabetes (READ-2 study). Ophthalmology. 2010;117:2146-51.

6. Do D, Nguyen Q, Khwaja A. Ranibizumab for Edema of the mAcula in Diabetes: 3-year outcomes and the need for prolonged frequent treatment. JAMA Ophthalmol. 2013;131:139-45.

7. Do D, Sepah Y, Boyer D, Callanan D, Gallemore R, Bennett M, et al. Month-6 primary outcomes of the READ-3 study (Ranibizumab for Edema of the mAcula in Diabetes-protocol 3 with high dose). Eye. 2105;29:1538-44.

8. Sepah Y, Sadiq M, Boyer D, Nguyen Q, Do D. Twenty-four-month outcomes of the Ranibizumab for Edema of the mAcula in Diabetes - protocol 3 with high dose (READ-3) study. Ophthalmology. 2016;123;12:2581-7.

9. Michaelides M, Kaines A, Hamilton R, Bunce C, Leslie D, Hykin P. A prospective randomized trial of bevacizumab or laser therapy in the management of diabetic macular edema (BOLT study): 12-month data, report 2. Ophthalmology. 2010;117:1078-86. 
10. Rajendram R, Fraser $S$, Kaines A. A 2-year prospective randomized controlled trial of intravitreal bevacizumab of laser therapy (BOLT) in the management of diabetic macular edema -24 -month data, report 3. Arch Ophthalmol. 2021;130:972-9.

11. Do D, Schmidt U, Gonzalez V, Yang K, Beckmann K, Heier J, et al. The DA VINCI Study: phase 2 primary results of VEGF Trap Eye in patients with diabetic macular edema. Ophthalmology. 2011;118: 1819-26.

12. Do D, Nguyen Q, Boyer D, Sandbrink R, Heier J. One year outcomes of the DA VINCI Study of VEGF Trap Eye in eyes with diabetic macular edema. Ophthalmology. 2012;119:1658-65.

13. Nguyen $Q$, Brown D, Marcus D, Boyer D, Patel S, Feiner L, et al. Ranibizumab for diabetic macular edema. Results from 2-phase III randomized trials: RISE \& RIDE. Ophthalmology. 2012;119:789-801.

14. Brown D, Nguyen Q, Marcus D, Boyer D, Patel S, Feiner L, et al. Longterm outcomes of ranibizumab therapy for diabetic macular edema: the 36 months results from 2-phase III randomized trials. Ophthalmology. 2013;120:2013-22.

15. Boyer D, Nguyen Q, Brown D, Basu K, Ehrlich J. Outcomes with as needed ranibizumab after initial monthly therapy. Long-term outcomes of the phase III RISE \& RIDE trials. Ophthalmology. 2015;122: 2504-13.
16. Elman M, Aiello L, Beck R, Bressler N, Bressler S, Edwards A, et al. Randomized trial evaluating ranibizumab plus prompt or deferred laser or triamcinolone plus prompt laser for diabetic macular edema. Ophthalmology. 2010;117:1064-77.

17. Elman M, Bressler N, Qin H, Beck R, Ferris F, Friedman S, et al. Expanded 2-year follow up of ranibizumab plus prompt or deferred laser or triamcinolone plus prompt laser for diabetic macular edema. Ophthalmology. 2011;118:609-14.

18. Elman M, Ayala A, Bressler N, Browning D, Flaxel C, Glassman A, et al Intravitreal ranibizumab for diabetic macular edema with prompt versus deferred laser treatment: 5-year randomized trial results. Ophthalmology. 2015;122:375-81.

19. Bressler S, Glassman A, Almukthar T, Bressler N, Ferris F, Googe J, et al. 5-Year outcomes of ranibizumab with prompt or deferred laser versus laser or triamcinolone plus deferred ranibizumab for diabetic macular edema. Am J Ophthalmol. 2016;164:57-68.

20. Wells J, Glassman A, Ayala A, Jampol L, Aiello L, Antoszyk A, et al. Aflibercept, bevacizumab or ranibizumab for diabetic macular edema. N Engl J Med. 2015;372:1193-203.

21. Wells J, Glassman A, Ayala A, Jampol L, Bressler N, Bressler S, et al. Aflibercept, bevacizumab or ranibizumab for diabetic macular edema: 2-year results from a comparative effectiveness randomized clinical trial. Ophthalmology. 2016;123:1351-9. 


\title{
¿Cuándo detener la terapia anti-VEGF? ¿Cuándo cambiar de anti-VEGF? ¿Cuándo usar láser en terapia anti-VEGF?
}

\author{
Patricio Rodríguez-Valdéz* \\ Instituto de Oftalmología y Ciencias Visuales, Tecnológico de Monterrey, Monterrey, Nuevo León, México
}

El Protocolo I de DRCR.net evaluó diferentes esquemas de tratamiento en pacientes con edema macular diabético (EMD) con un seguimiento de 5 años, y demostró el beneficio del tratamiento con ranibizumab $(0.5 \mathrm{mg})$. Sin embargo, no todos los pacientes en los grupos tratados con $0.5 \mathrm{mg}$ de ranibizumab tuvieron una respuesta favorable, lo cual es común en la práctica clínica'.

Numerosos estudios han buscado factores predictivos de la respuesta al tratamiento antiangiogénico evaluando diferentes variables como biomarcadores en pruebas de imagen, análisis de características basales, etc. Entre ellos, un análisis post hoc de los resultados del Protocolo I de DRCR.net (análisis EARLY) que evaluó la relación entre la respuesta temprana al tratamiento con ranibizumab $0.5 \mathrm{mg}$ (respuesta visual en la semana 12, después de tres inyecciones de ranibizumab con intervalos de 1 mes) con el resultado visual a 1, 2 y 3 años en pacientes con tratamiento mantenido con ranibizumab cumpliendo los criterios de retratamiento, encontró que la respuesta al tratamiento después de las primeras tres inyecciones podía predecir la respuesta a largo plazo. En promedio, los pacientes con una ganancia < 5 letras de visión después de las tres primeras inyecciones mensuales de ranibizumab no obtuvieron una mayor ganancia de letras de visión al continuar el tratamiento con ranibizumab por 1, 20

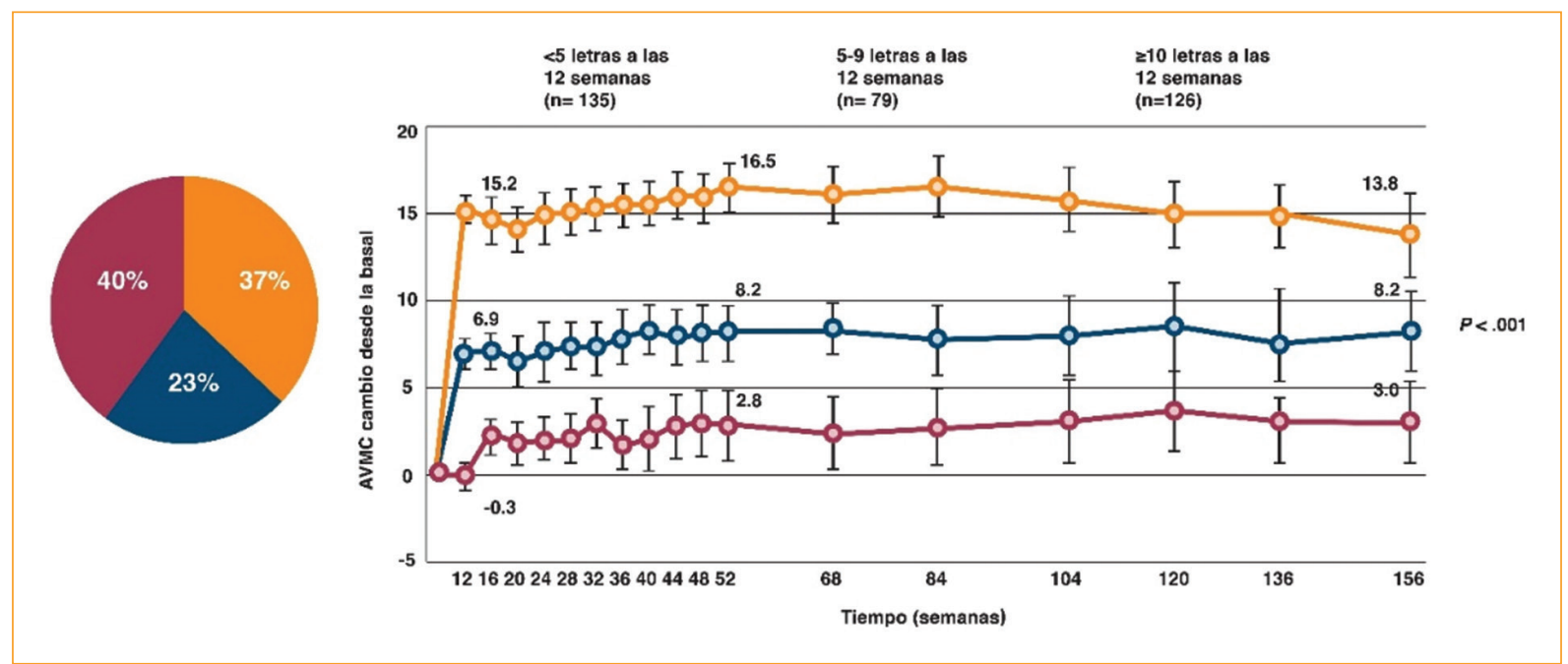

Figura 1. Resultados del análisis EARLY del Protocolo I de DRCR.net. Los ojos fueron agrupados según la respuesta visual después de las primeras tres inyecciones de ranibizumab con un intervalo de 1 mes. El 40\% presentaron una ganancia < 5 letras ETDRS, el $23 \%$ entre 5 y 9 letras, y el $37 \% \geq 10$ letras. Las ganancias visuales promedio por grupo se mantuvieron estables a lo largo del tiempo. AVMC: agudeza visual mejor corregida. 
3 años (figura 1). Más aún, este patrón de «pobres respondedores» fue el más común, representando el $40 \%$ de los pacientes tratados (figura 1$)^{2}$.

Esto no quiere decir que un paciente en específico que ganara $<5$ letras después de las tres primeras inyecciones no tuviera posibilidades de mejoría visual con el tratamiento mantenido con ranibizumab. La figura 2 muestra el comportamiento de este grupo de pacientes a lo largo del tiempo. La posibilidad de que un paciente de este grupo con pobre respuesta inicial lograra una buena respuesta funcional (ganancia de $\geq$ 10 letras de visión) con el tratamiento mantenido con ranibizumab fue de alrededor del $25 \%$, mientras que el $50 \%$ nunca tendrán mejoría visual.

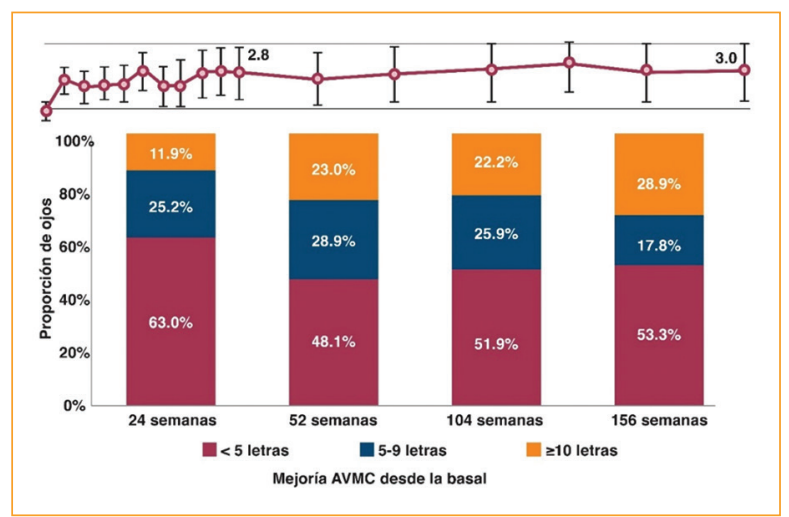

Figura 2. Resultados del análisis EARLY del Protocolo I de DRCR.net. Comportamiento a lo largo del tiempo (3 años) de los pacientes en tratamiento mantenido con ranibizumab que tuvieron una pobre respuesta visual a la semana 12. Alrededor del $50 \%$ nunca mejoraron $>5$ letras, mientras que el $25 \%$ mejoraron de 5 a 9 letras y el otro $25 \%$ mejoraron $\geq 10$ letras. AVMC: agudeza visual mejor corregida.
El análisis EARLY solo incluyó pacientes en tratamiento con ranibizumab $0.5 \mathrm{mg}$, pero un análisis similar de los pacientes del protocolo T de DRCR.net reveló un comportamiento similar con bevacizumab $1.25 \mathrm{mg}$ y aflibercept $2.0 \mathrm{mg}$ (figuras 3 y 4), por lo que se puede suponer que este es un patrón que aplica para los antiangiogénicos en general ${ }^{3}$.

Si bien hasta ahora se ha hablado únicamente de los resultados visuales de estos estudios, los datos acerca de los resultados anatómicos del Protocolo T de DRCR.net demuestran que el tratamiento mantenido con cualquiera de los agentes antiangiogénicos conlleva una disminución progresiva del grosor macular central (figura 5), que no se relaciona necesariamente con una mejoría visual ${ }^{4}$.

\section{Cambio de tratamiento}

Los resultados presentados anteriormente indican la baja probabilidad de que un paciente determinado con una pobre respuesta inicial al tratamiento (semana 12) pueda obtener una ganancia visual con el tratamiento mantenido con el mismo agente antiangiogénico, por lo cual este puede ser el momento adecuado para considerar un cambio o una combinación de tratamientos buscando el beneficio del paciente.

\section{Cambiar de antiangiogénico (switch)}

Existen numerosos reportes de casos y pequeñas series que han evaluado el efecto del cambio de agente antiangiogénico en ojos con respuestas subóptimas al tratamiento. Algunos de los resultados de estos reportes se muestran en la figura $6^{5-9}$.
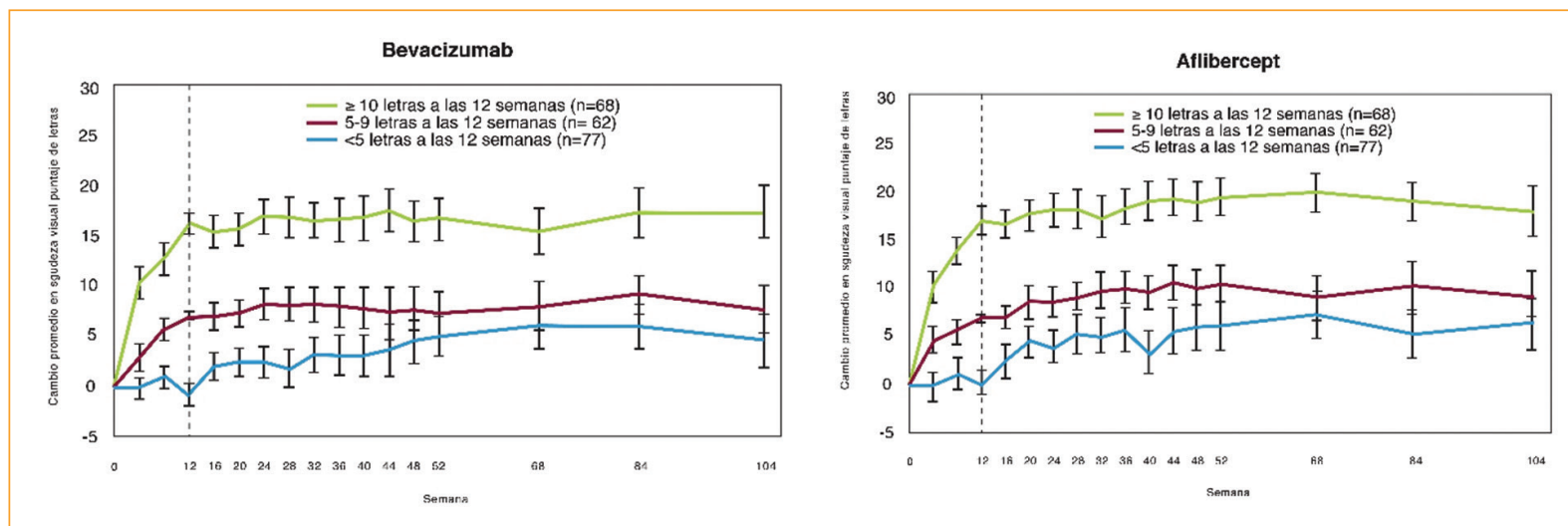

Figura 3. Resultados del análisis post hoc del Protocolo T de DRCR.net. Comportamiento a lo largo del tiempo (2 años) de los pacientes en tratamiento mantenido con bevacizumab $1.25 \mathrm{mg}$ y aflibercept 2.0 mg agrupados según la respuesta inicial (12 semanas). 


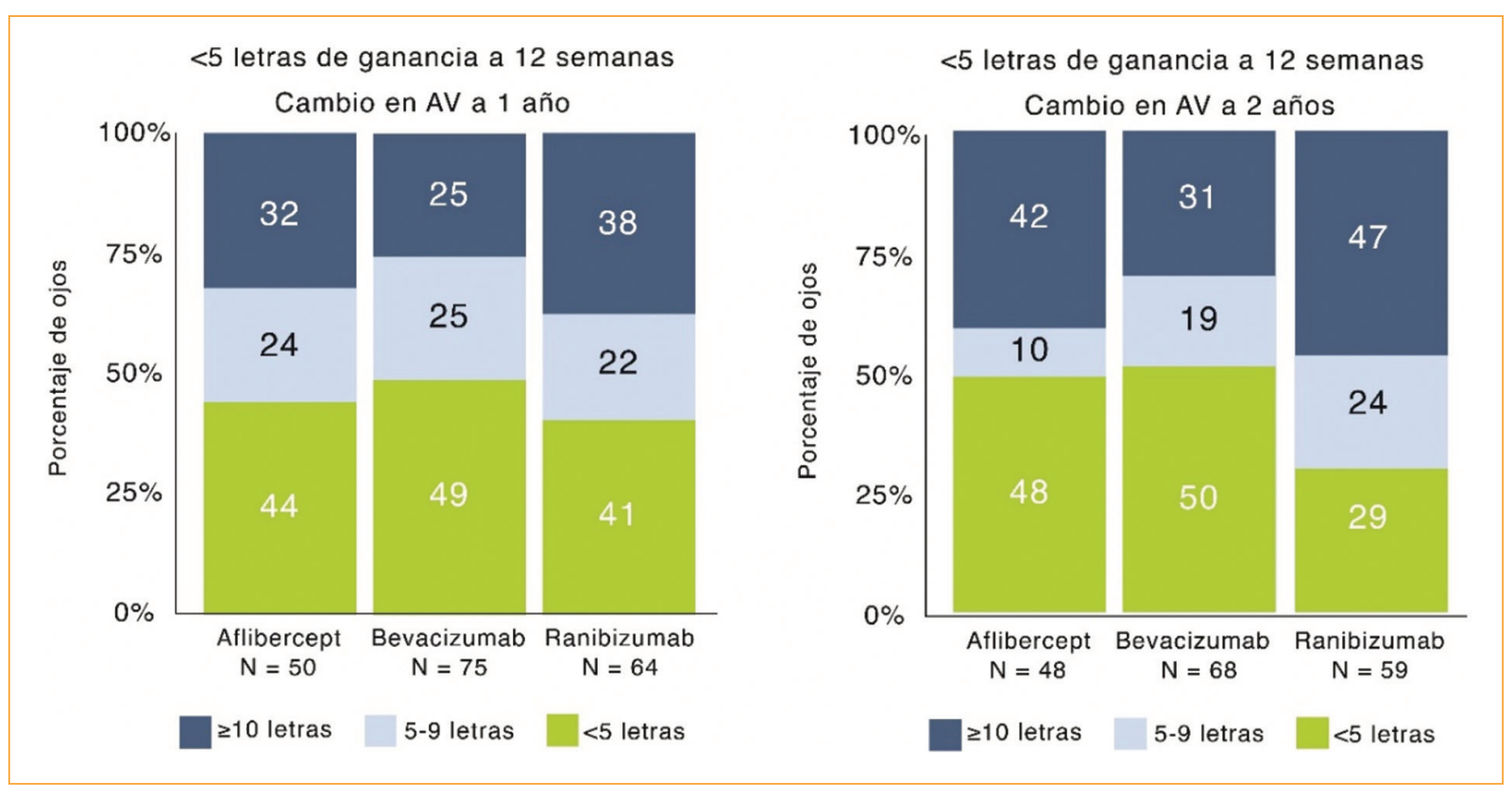

Figura 4. Resultados del análisis post hoc del Protocolo T de DRCR.net. Comportamiento a lo largo del tiempo (2 años) de los pacientes en tratamiento mantenido con bevacizumab $1.25 \mathrm{mg}$, aflibercept $2.0 \mathrm{mg}$ y ranibizumab $0.5 \mathrm{mg}$ con pobres respuestas visuales a la semana 12. La mitad de los pacientes se mantuvieron con ganancias $<5$ letras a 2 años del inicio del tratamiento en los grupos de bevacizumab y aflibercept. AV: agudeza visual.

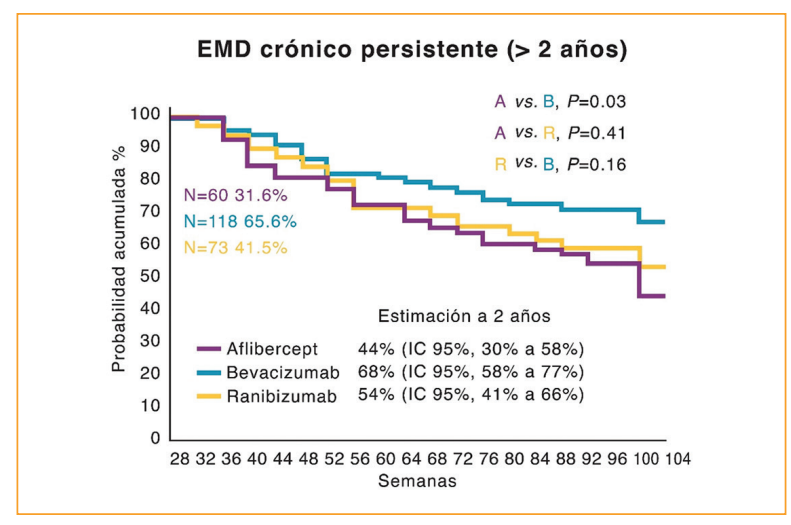

Figura 5. Resultados del análisis post hoc del Protocolo T de DRCR.net. Persistencia de edema macular residual (por tomografía de coherencia óptica) a lo largo del tiempo en ojos con edema macular residual en la semana 24 de tratamiento. El edema macular residual a los 6 meses de tratamiento se resolvió en el $56 \%$, el $46 \%$ y el $32 \%$ de los ojos tratados con aflibercept $2.0 \mathrm{mg}$, ranibizumab $0.5 \mathrm{mg}$ y bevacizumab $1.25 \mathrm{mg}$, respectivamente, después de 2 años de tratamiento continuo. EMD: edema macular diabético.

Sin embargo, la mayoría de estos reportes son no comparativos y carecen de grupo control, y además asumen que no habrá mejoría si se continúa con el mismo agente antiangiogénico. En un análisis realizado por Ferris, et al. ${ }^{10}$ se cambió de un medicamento $A$ a un medicamento B si los pacientes cumplían con las siguientes características: agudeza visual menor de 20/40, persistencia de edema macular y menos de 5 letras de ganancia después de tres inyecciones mensuales de ranibizumab $0.5 \mathrm{mg}$. Tras el cambio del medicamento A al medicamento $B$ (switch), los pacientes mejoraron en promedio 5 letras de visión y tuvieron una disminución en el grosor macular central de $76 \mu \mathrm{m}$ (figura 7). Parece que este switch tuvo un resultado favorable, pero el medicamento A era ranibizumab $0.5 \mathrm{mg}$ y el medicamento $B$ también era ranibizumab $0.5 \mathrm{mg}$, se trataba de pacientes del protocolo I de DRCR.net que cumplieron los criterios de inclusión para este análisis, y en realidad era una continuación de tratamiento y no un switch, recalcando la importancia de tener un grupo control cuando se evalúa un switch entre agentes farmacológicos.

En resumen, no existe evidencia clínica de calidad que avale que el cambio de agente antiangiogénico tenga un efecto favorable en los pacientes con respuestas subóptimas al tratamiento.

\section{Adición de fotocoagulación con láser macular}

En uno de los grupos de estudio del protocolo I de DRCR.net se evaluó la combinación de ranibizumab y fotocoagulación con láser macular (siguiendo los lineamientos modificados del ETDRS [Early Treatment Diabetic Retinopathy Study]) frente a la monoterapia farmacológica 


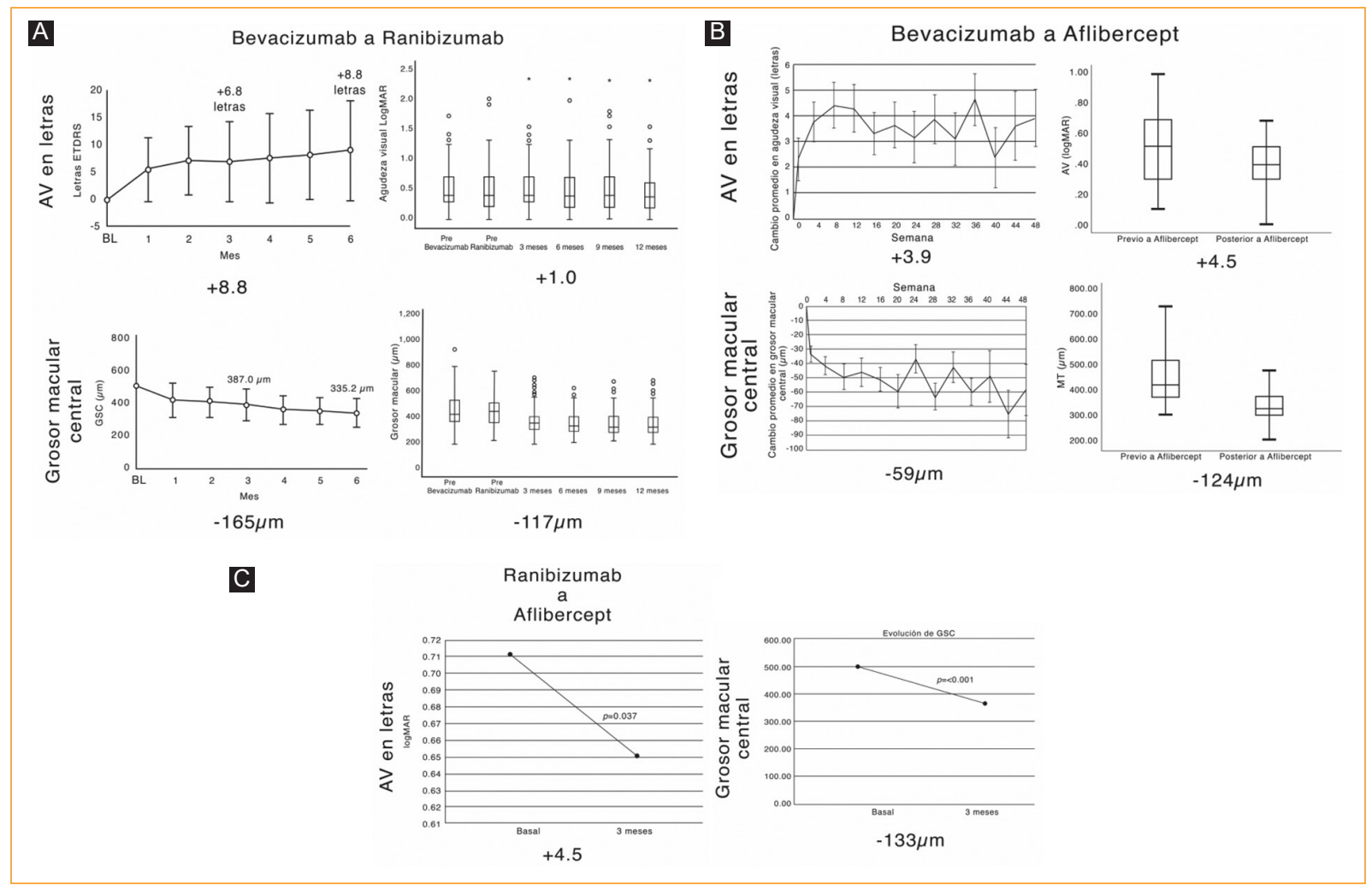

Figura 6. Resultados de series de casos. Existe beneficio visual y anatómico al cambiar entre agentes antiangiogénicos (switch). A: de bevacizumab a ranibizumab. B: de bevacizumab a aflibercept. C: de ranibizumab a aflibercept. Nótese la ausencia de grupo control. AV: agudeza visual; ETDRS: Early Treatment Diabetic Retinopathy Study; GSC: grosor del subcampo central; MT: grosor macular.

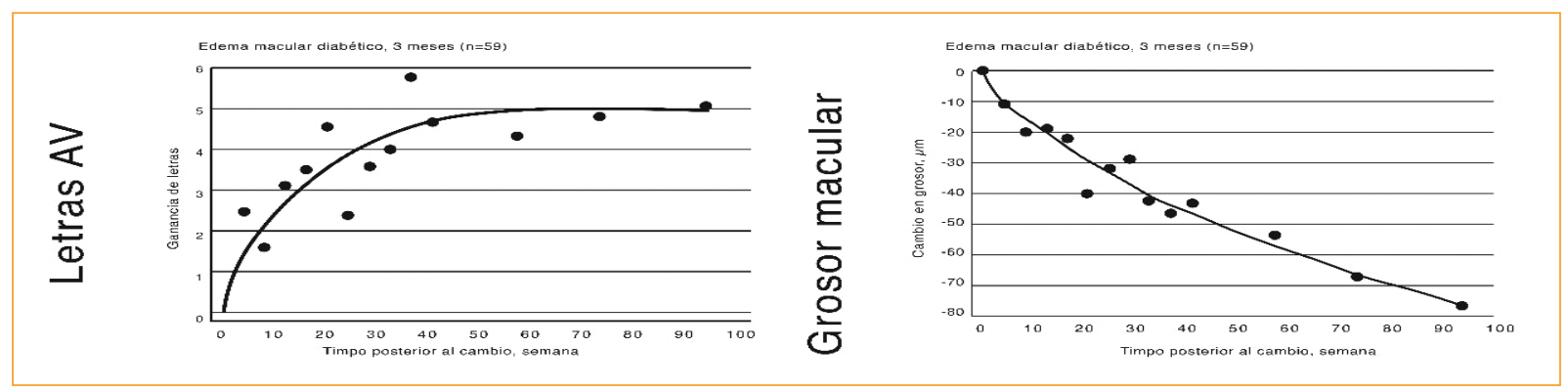

Figura 7. Resultados en agudeza visual (AV) y grosor macular después del engaño de switch. Ejemplifica la falta de evidencia en el cambio entre medicamentos antiangiogénicos al carecer de grupo control.

(aunque el $28 \%$ de estos pacientes sí recibieron fotocoagulación con láser durante el seguimiento). Los resultados en ese grupo combinado no demostraron ningún beneficio sobre la monoterapia con ranibizumab, lo cual sugiere que la adición de fotocoagulación con láser macular no otorga ningún beneficio en los pacientes con EMD (figura 8)'.

Numerosos estudios, incluyendo el TREX-DME, fallaron en demostrar beneficio visual o anatómico con la adición de láser navegado micropulsado amarillo (577 nm) guiado por angiografía a la monoterapia con un antiangiogénico (figura 9) ${ }^{11}$.
En conclusión, no existe evidencia clínica que indique beneficio de la adición de fotocoagulación con láser macular en ninguna de sus variantes a la monoterapia antiangiogénica.

\section{Adición de fotocoagulación con láser periférica en áreas de no perfusión por angiografía con fluoresceína}

Ante la suposición de que añadir fotocoagulación con láser periférica a las áreas de no perfusión capilar 


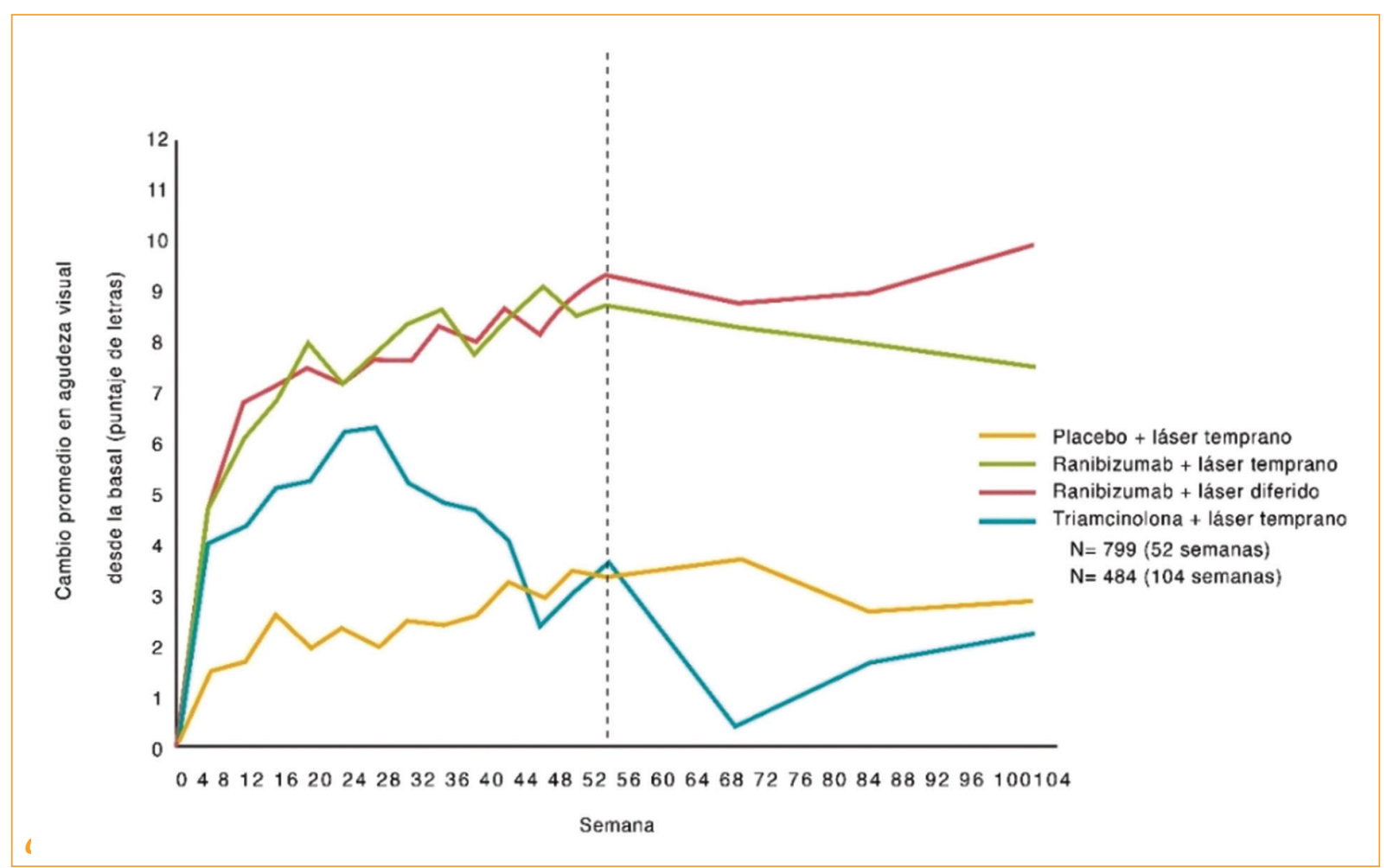

Figura 8. Comparación entre los cuatro grupos del Protocolo I de DRCR.net. Se observa la similitud de resultados entre el grupo de ranibizumab con láser inmediato (obligatorio) y el grupo de ranibizumab con láser diferido (opcional después de 6 meses; solo el $28 \%$ de los ojos de este grupo requirieron láser de rescate).

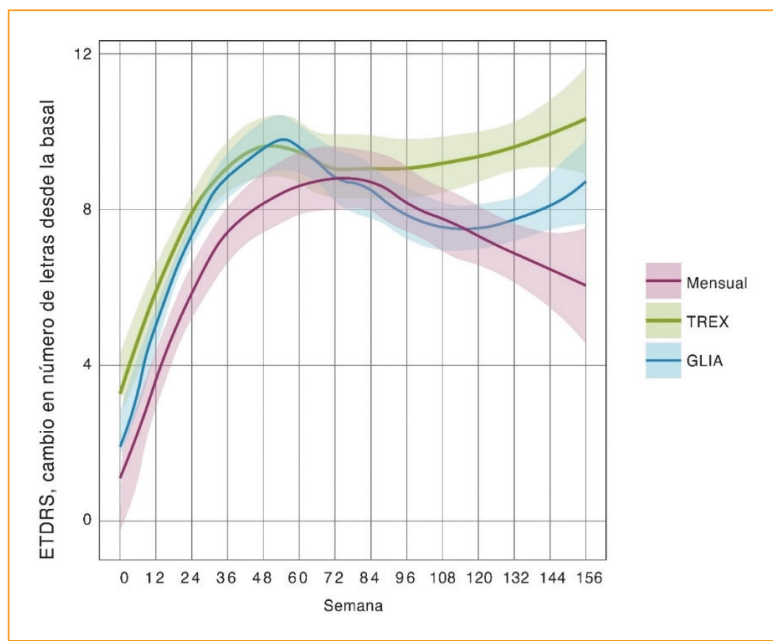

Figura 9. Resultados visuales del estudio TREX-DME. No hubo diferencia estadísticamente significativa $(p=0.60)$ entre la monoterapia mantenida mensual y el protocolo de tratar y extender combinado con láser micropulsado navegado amarillo guiado (GLIA) o no guiado (TREX) por angiografía. ETDRS: Early Treatment Diabetic Retinopathy Study.

determinadas por angiografía de retina con fluoresceína pudiera disminuir la cantidad de factor de crecimiento endotelial vascular (VEGF, vascular endothelial growth factor), y de esa manera ayudar en el tratamiento del EMD, se llevó a cabo el estudio DAVE, un pequeño estudio controlado que comparó la monoterapia con ranibizumab con la combinación de ranibizumab y fotocoagulación con láser periférica a las áreas no perfundidas por angiografía de retina con fluoresceína. Los resultados demuestran que no existe beneficio al agregar a la monoterapia con ranibizumab láser periférico a las áreas no perfundidas en la angiografía, tanto en los resultados visuales como en los anatómicos (figura 10), y tampoco en el número de inyecciones requeridas (figura 11) ${ }^{12}$.

\section{Cambio a implante de dexametasona}

El protocolo $U$ de DRCR.net es un estudio diseñado para evaluar el efecto de la adición (combinación) de un implante de dexametasona al tratamiento mantenido con ranibizumab en pacientes con edema macular persistente posterior a por lo menos tres inyecciones mensuales de ranibizumab, en comparación con la monoterapia mantenida con ranibizumab. Aunque en el diseño original del estudio se estableció que no se incluirían pacientes fáquicos para no confundir los resultados visuales por la 

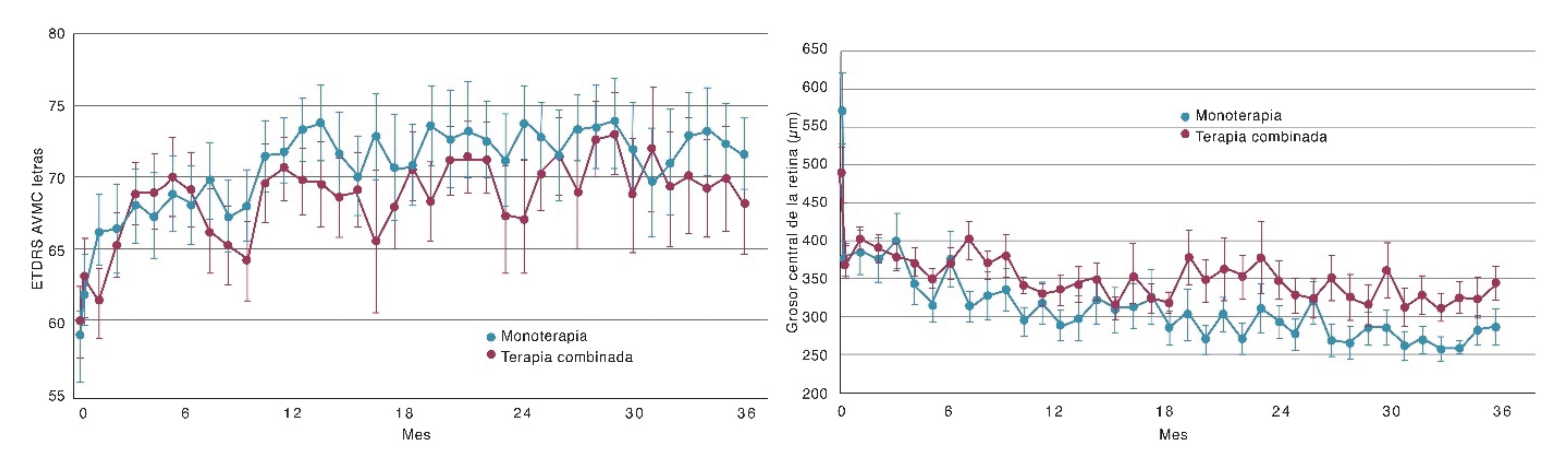

Figura 10. Resultados del estudio DAVE, visuales y anatómicos, con monoterapia con ranibizumab (azul) y con ranibizumab más láser periférico al área de no perfusión capilar por angiografía con fluoresceína (rojo). No hubo diferencia estadísticamente significativa. La monoterapia fue superior en cuanto a letras de agudeza visual ganadas y disminución de grosor macular central. AVMC: agudeza visual major corregida; ETDRS: Early Treatment Diabetic Retinopathy Study.

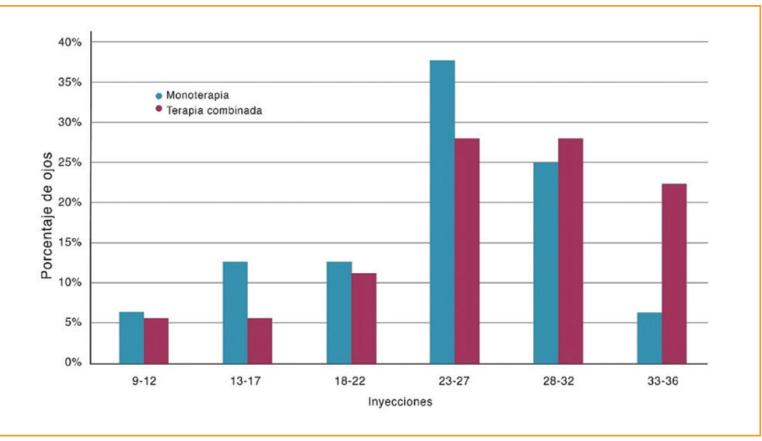

Figura 11. Resultados del estudio DAVE. Exposición a tratamiento (cantidad de inyecciones requeridas) en ojos tratados con monoterapia con ranibizumab (azul) y terapia combinada de ranibizumab y fotocoagulación láser a las áreas de no perfusión capilar por angiografía con fluoresceína (rojo). Los pacientes con monoterapia requirieron menos inyecciones.

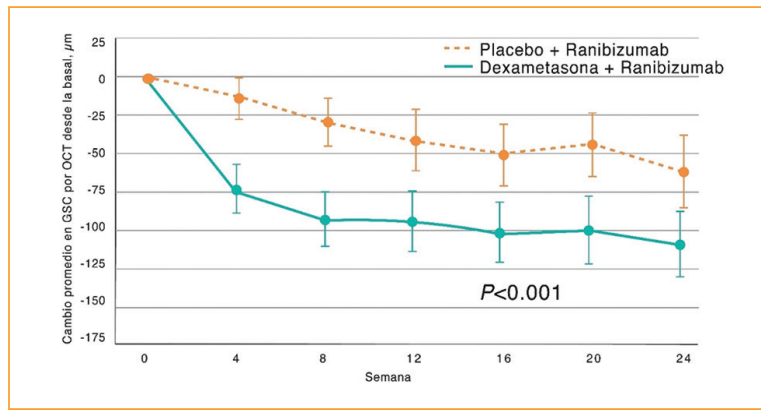

Figura 12. Resultados anatómicos del Protocolo U de DRCR.net. Cambio en el grosor macular central en pacientes con edema macular residual en tratamiento mantenido con ranibizumab (línea azul claro) frente a ranibizumab e implante de dexametasona (línea verde oscuro). Los pacientes tratados con adición de implante de dexametasona tuvieron una disminución de $100 \mu \mathrm{m}$ mayor que los pacientes en tratamiento mantenido ( $p$ < 0.001). GSC: grosor del subcampo central; OCT: tomografía de coherencia óptica.

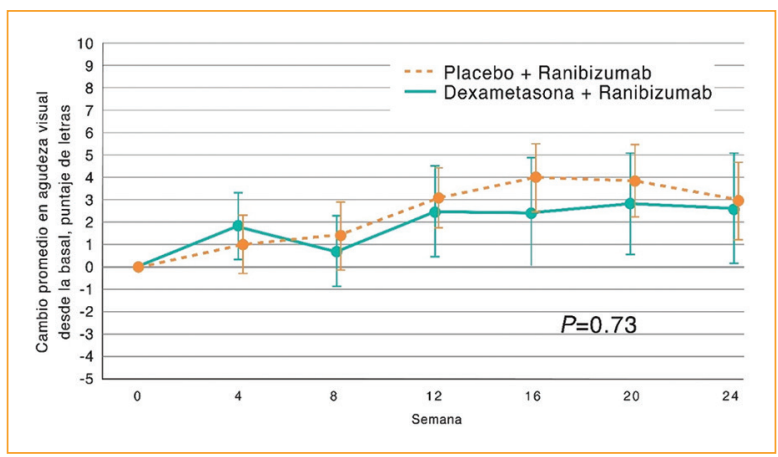

Figura 13. Resultados visuales del Protocolo U de DRCR. net. Cohorte completa. Cambio en agudeza visual promedio en pacientes con edema macular residual en tratamiento mantenido con ranibizumab (línea azul claro) o con ranibizumab más implante de dexametasona (línea verde oscuro). No se observa diferencia entre ambos grupos $(p=0.73)$.

formación de catarata, este criterio fue enmendado debido al lento reclutamiento de pacientes. El estudio demostró una mejoría estadísticamente significativa y clínicamente relevante del edema macular al añadir el implante de dexametasona al tratamiento con ranibizumab. Los ojos que recibieron la combinación tuvieron una disminución de $100 \mu \mathrm{m}$ de grosor macular central más que los ojos que continuaron con la monoterapia (figura 12) $)^{13}$.

En cuanto a los resultados visuales de la cohorte completa, no se demostró un beneficio en ganancia de letras ETDRS (figura 13).

Sin embargo, este resultado puede verse alterado por varios factores, entre ellos el alto porcentaje de pacientes fáquicos incluido (60\%) y el alto porcentaje de pacientes con buena agudeza visual (el $56 \%$ con $20 / 50$ 0 mejor), lo que da un efecto techo, y con buenos 


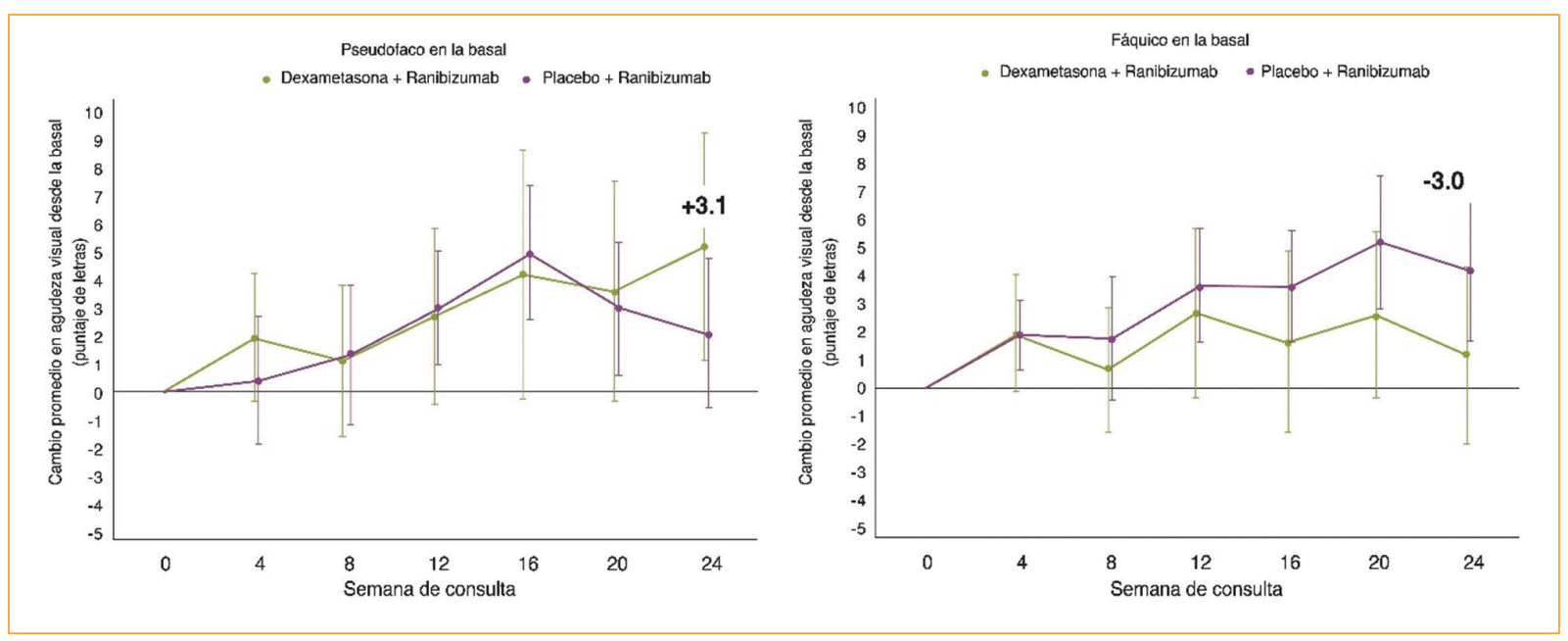

Figura 14. Resultados visuales del Protocolo U de DRCR.net por subgrupos. Los pacientes pseudofacos con edema macular residual en quienes se añadió el implante de dexametasona tuvieron una ganancia de +3.1 letras, frente a una pérdida de 3 letras en los pacientes fáquicos tratados también con implante de dexametasona; una diferencia de +6.1 determinada solo por el estado del cristalino $(p=0.08)$.
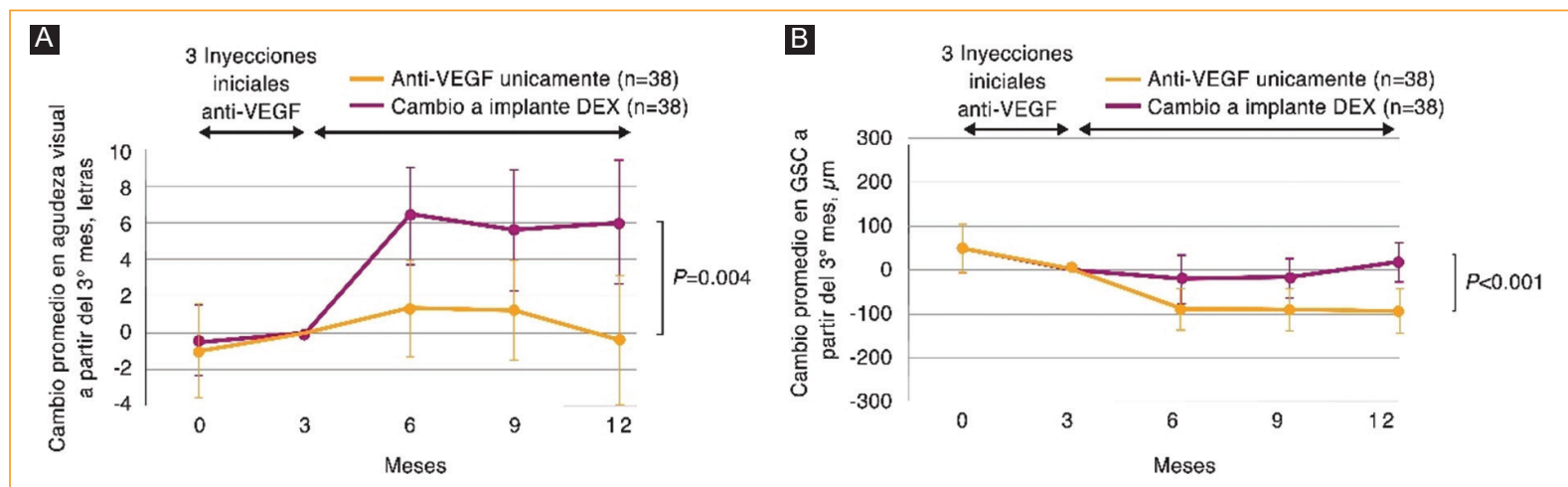

Figura 15. A: resultados visuales a 12 meses del estudio Shall we stay or shall we switch?, que muestran una ganancia de +6.0 letras en el grupo con switch a dexametasona (DEX), mientras que el grupo con terapia mantenida con antiangiogénico (grupo control) no tuvo mejoría visual $(p=0.004)$. B: resultados anatómicos del mismo estudio, que muestran que los pacientes con switch a dexametasona (DEX) redujeron el del subcampo (GSC) $<100 \mu \mathrm{m}$ más que el grupo control $(p<0.001)$. VEGF: vascular endothelial growth factor.

grosores maculares (el $50 \%$ con $<350 \mu \mathrm{m}$ ). En pocas palabras, los pacientes incluidos no eran los mejores para encontrar diferencias entre los grupos. Esto se puede ver reflejado al analizar por subgrupos los pacientes fáquicos y pseudofáquicos tratados con implante de dexametasona, pues hay una diferencia de +6 letras a favor de los pacientes pseudofáquicos (figura 14).

El estudio Shall we stay or shall we switch? del International Retina Group es un estudio controlado, retrospectivo, de la vida real, que analizó el efecto de reemplazar la terapia antiangiogénica con implante de dexametasona en pacientes vírgenes a tratamiento que tuvieron una pobre respuesta al tratamiento con tres dosis mensuales de antiangiogénico. Sus conclusiones demuestran un mejor resultado visual (+6 letras) y anatómico $(-100 \mu \mathrm{m})$ con el cambio temprano (12 semanas) a implante de dexametasona en comparación con el tratamiento mantenido con antiangiogénicos (grupo control) (figura 15) ${ }^{14}$. 


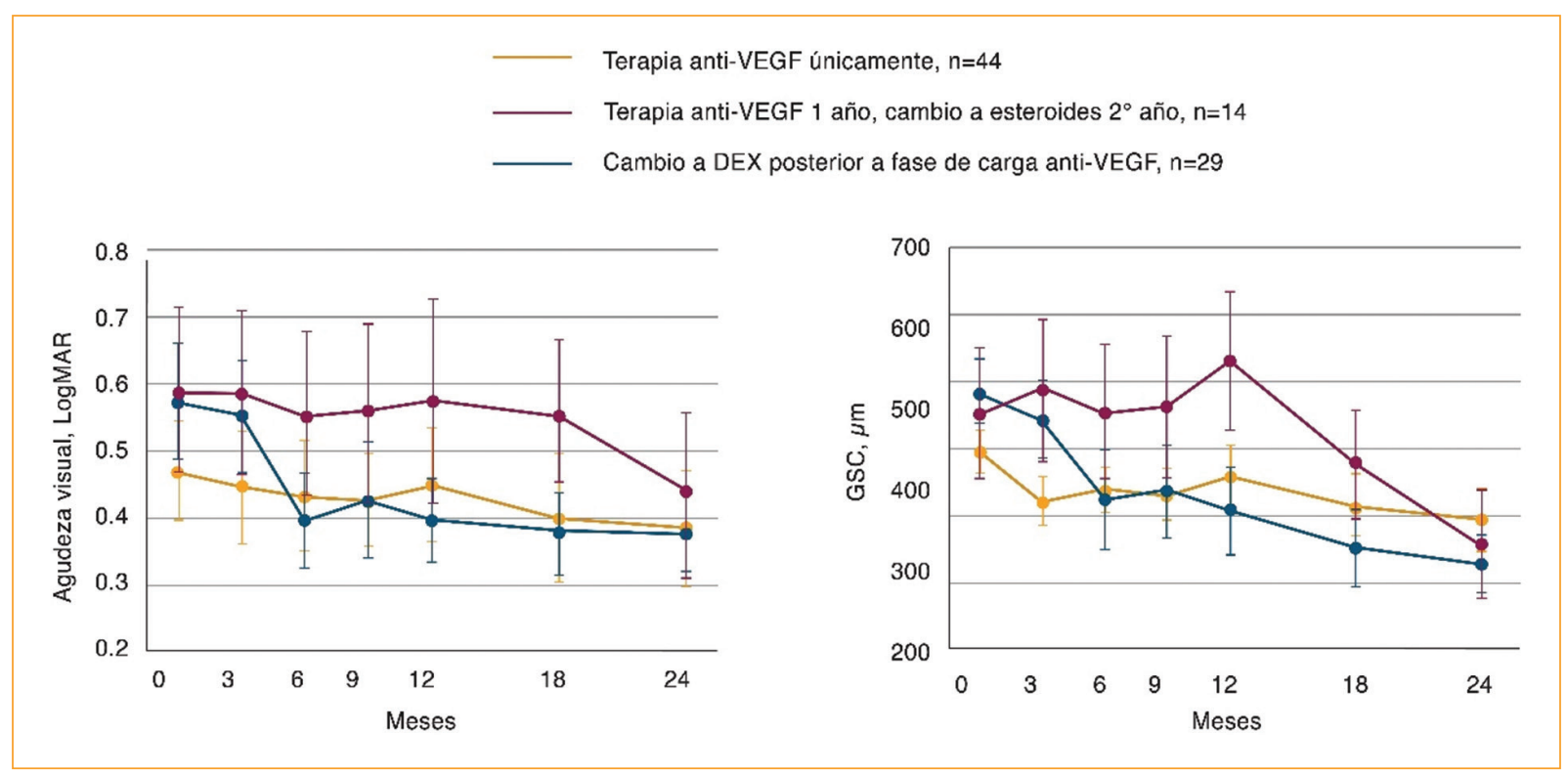

Figura 16. Resultados visuales a 24 meses del estudio de extensión Shall we stay or shall we switch? que muestran que el beneficio anatómico y visual obtenido por el switch temprano (12 semanas) a implante de dexametasona (DEX) durante el primer año fue mantenido durante el segundo año. Los pacientes con switch tardío a implante de dexametasona (12 meses; línea color magenta) también presentaron beneficio, pero no alcanzó los resultados visuales de los pacientes con switch temprano (12 semanas). GSC: grosor del subcampo central; VEGF: vascular endothelial growth factor.

Además, en su estudio de extensión se observó que, aunque el switch tardío (12 meses) produjo un beneficio visual y anatómico en pacientes con pobre respuesta al antiangiogénico, el resultado fue mejor en los pacientes con switch temprano (12 semanas) (figura 16) ${ }^{15}$.

En conclusión, debe considerarse el cambio de tratamiento en todo paciente con una respuesta subóptima después de tres inyecciones mensuales de antiangiogénico. No hay evidencia de que la adición de láser en sus varias modalidades mejore los resultados visuales, ni de que el cambio de agente antiangiogénico sea benéfico para el paciente. La adición de un implante de dexametasona brinda un beneficio visual y anatómico en los pacientes con pobre respuesta al tratamiento.

\section{Bibliografía}

1. Elman M, Aiello L, Beck R, Bressler N, Bressler, Edwards A, et al. Randomized trial evaluating ranibizumab plus prompt or deferred laser or triamcinolone plus prompt laser for diabetic macular edema. Ophthalmology. 2010;117:1064-77

2. Gonzalez V, Campbell J, Holekamp N, Kiss S, Loewenstein A, Augustin $A$, et al. Early and long-term responses to anti-vascular endothelial growth factor therapy in diabetic macular edema: analysis of Protocol I data. Am J Ophthalmol. 2016:172:72-9.

3. Bressler N, Beaulieu W, Maguire M, Glassman A, Blinder K, Bressler S, et al. Early response to anti-vascular endothelial growth factor and twoyear outcomes among eyes with diabetic macular edema in Protocol T. Am J Ophthalmol. 2018;195:93-100.
4. Bressler N, Beaulieu W, Glassman A, Blinder K, Bressler S, Jampol L, et al. Persistent macular thickening following intravitreous aflibercept, bevacizumab, or ranibizumab for central-involved diabetic macular edema with vision impairment: a secondary analysis of a randomized clinical trial. JAMA Ophthalmol. 2018;136:257-69.

5. Dhoot D, Pieramici D, Nasir M, Castellarin A, Couvillion, See R, et al. Residual edema evaluation with ranibizumab $0.5 \mathrm{mg}$ and $2.0 \mathrm{mg}$ formulations for diabetic macular edema (REEF study). Eye (Lond). 2015;29:534-41.

6. Ehrlich R, Pokroy R, Segal O, Goldstein M, Pollack A, Hanhart J, et al Diabetic macular edema treated with ranibizumab following bevacizumab failure in Israel (DERBI study). Eur J Ophthalmol. 2019;29:229-33.

7. Bahrami B, Hong T, Schlub T, Chang A. Aflibercept for persistent diabetic macular edema: forty-eight-week outcomes. Retina. 2019;39:61-8.

8. Laiginhas R, Silva M, Rosas V, Penas S, Fernandes V, Rocha A, et al. Aflibercept in diabetic macular edema refractory to previous bevacizumab: outcomes and predictors of success. Graef Arch Clin Exp Ophthalmol. 2018;256:83-9.

9. Mira F, Paulo M, Henriques F, Figueira J. Switch to aflibercept in diabetic macular edema patients unresponsive to previous anti-VEGF therapy. J Ophthalmol. 2017:2017:5632634.

10. Ferris F, Maguire M, Glassman A, Ying G, Martin D. Evaluating effects of switching anti-vascular endothelial growth factor drugs for age-related macular degeneration and diabetic macular edema. JAMA Ophthalmol. 2017;135:145-99.

11. Payne J, Wykoff C, Clark W, Bruce B, Boyer D, Brown D. Long-term outcomes of treat-and-extend ranibizumab with and without navigated laser for diabetic macular oedema: TREX-DME 3-year results. Br J Ophthalmol 2021;105:253-7.

12. Brown D, Ou W, Wong T, Kim R, Croft D, Wykoff C. Targeted retinal photocoagulation for diabetic macular edema with peripheral retinal nonperfusion: three-year randomized DAVE trial. Ophthalmology. 2018;125:683-90.

13. Ung C, Moussa K, Yonekawa Y. Combination treatment of diabetic macular edema with antivascular endothelial growth factor and steroids: analyses of the DRCR.net protocol U. Ann J Ophthalmic Clin Trials 2018;1:1-5.

14. Busch C, Zur D, Fraser S, Lains I, Santos A, Lupidi M, et al. Shall we stay, or shall we switch? Continued anti-VEGF therapy versus early switch to dexamethasone implant in refractory diabetic macular edema. Acta Diabetol. 2018:55:789-96.

15. Busch C, Fraser S, Iglicki M, Lupidi M, Couturier A, Chaikitmongkol V, et al. Real world outcomes on non-responding diabetic macular edema treated with continued anti-VEGF therapy versus early switch to dexamethasone implant: 2-year results. Acta Diabetol. 2019;56:1341-50. 


\title{
Pacientes con edema macular diabético no respondedores a terapia antiangiogénica (análisis del estudio EARLY)
}

\author{
Juan M. Jiménez-Sierra* \\ Asociación para Evitar la Ceguera en México (APEC), Ciudad de México, México
}

La terapia antiangiogénica (anti-VEGF [Vascular Endothelial Growth Factor]) es la modalidad terapéutica más utilizada en la actualidad para el tratamiento del edema macular diabético (EMD). También se considera por la mayoría de los especialistas en retina como el tratamiento de primera línea en esta patología ${ }^{1}$.

Si bien un porcentaje de pacientes presenta muy buena respuesta a este tratamiento, existen muchos ojos que, a pesar de múltiples inyecciones de antiVEGF, logran poca o nula mejoría en la agudeza visual, y en algunas ocasiones muestran disminución del grosor macular central medido por tomografía de coherencia óptica, pero sin mejoría de la agudeza visual, y otros pacientes no presentan cambio en el grosor macular ${ }^{2,3}$.

El primer problema es que no existe una clara definición para que los pacientes sean catalogados como «no respondedores», y además existe discrepancia en el número de inyecciones para poder afirmar quién será no respondedor a la terapia anti-VEGF; este aspecto sigue siendo tema de controversia entre los especialistas de retina ${ }^{4}$.

Ante un paciente con EMD que ha recibido tres inyecciones mensuales consecutivas con algún agente anti-VEGF y no responde de forma adecuada, surge una pregunta: ¿cuál es el siguiente paso?

Las posibilidades serían":

- Cambiar a otro anti-VEGF.

- Continuar con el mismo anti-VEGF durante 3 meses más.
- Cambiar a un esteroide (implante de dexametasona intravítrea).

- Aplicar láser focal.

En este capítulo nos basaremos principalmente en los resultados del estudio EARLY, ya que es el más específico para responder esta pregunta y analiza la predicción de la respuesta a largo plazo considerando las tres primeras inyecciones ${ }^{1}$.

El estudio Protocolo I, multicéntrico, de fase III, que incluyó 691 pacientes (854 ojos con EMD), fue llevado a cabo por el DRCR.net. Un análisis post hoc de sus resultados (estudio EARLY) evaluó la relación entre la respuesta temprana al tratamiento con ranibizumab $0.5 \mathrm{mg}$ (respuesta visual en la semana 12, después de tres inyecciones de ranibizumab con intervalos de 1 mes) y el resultado visual a 1, 2 y 3 años en pacientes

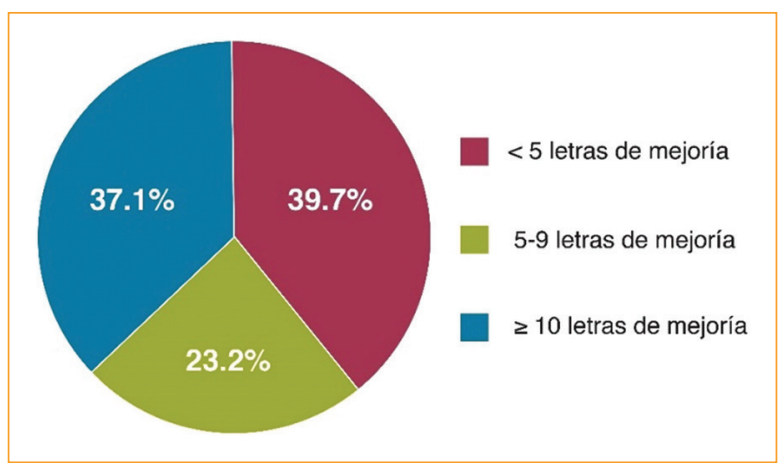

Figura 1. Resultados del Protocolo I a 12 semanas, estratificados por grupos de mejoría. 


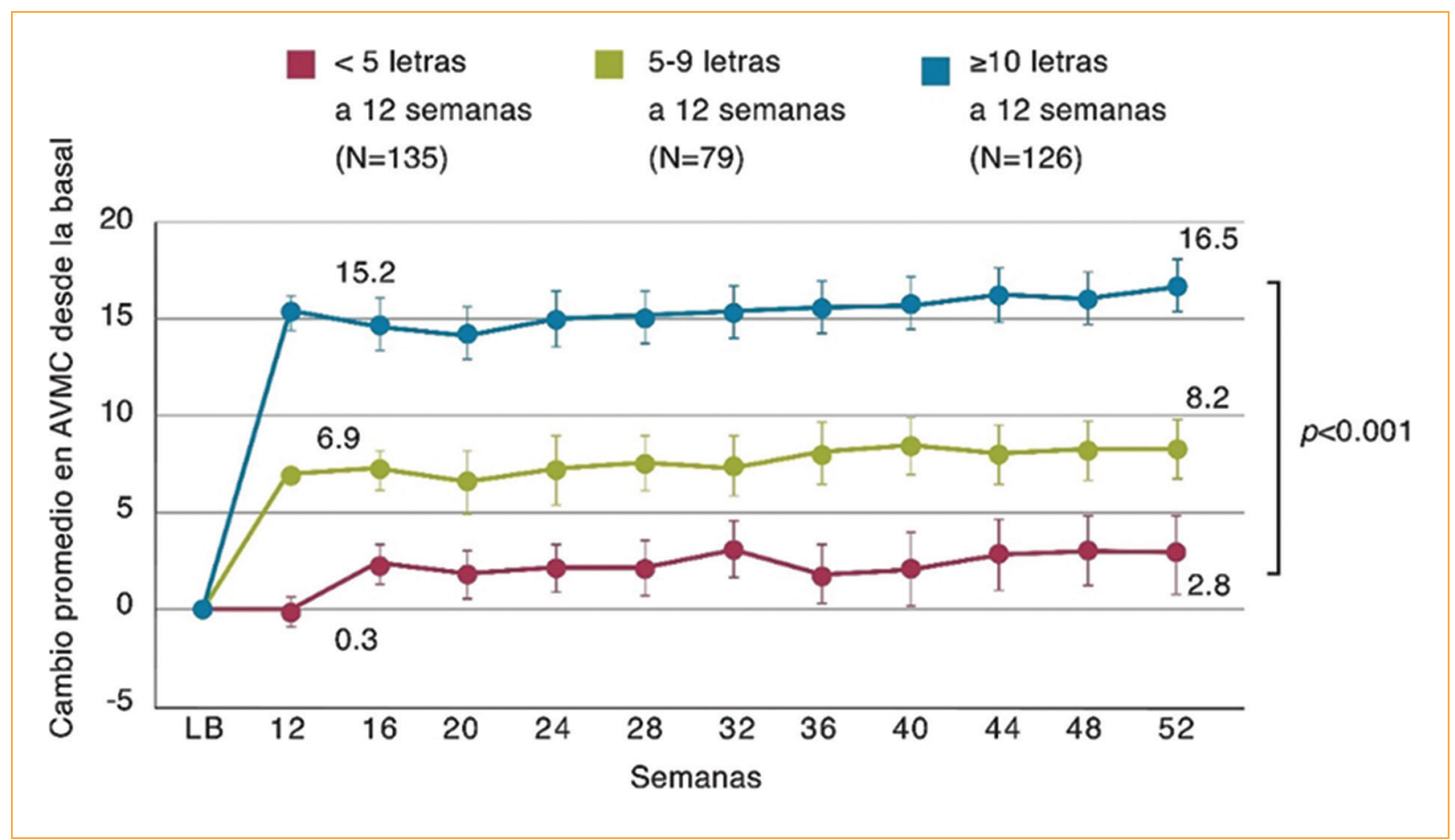

Figura 2. Respuesta a 12 meses. En color rojo se muestra la respuesta a las 12 semanas (< 5 letras); en verde, mejoría inicial entre 5 y 9 letras, y que tienden a mantener esa mejoría; y en azul, buena mejoría inicial a las 12 semanas > 10 letras, y que tienden a mantener una buena agudeza visual mejor corregida (AVMC) a los 12 meses.

con tratamiento mantenido con ranibizumab cumpliendo los criterios de retratamiento, y encontró que la respuesta al tratamiento después de las primeras tres inyecciones podía predecir la respuesta a largo plazo'.

El objetivo del análisis fue evaluar si la respuesta al anti-VEGF después de tres inyecciones (12 semanas) podía predecir la respuesta a largo plazo en el EMD, comparando la respuesta a las 3 semanas y el resultado a los 12 meses. Los resultados se estratificaron en tres grupos de acuerdo con la agudeza visual corregida utilizando la cartilla del ETDRS (Early Treatment Diabetic Retinopathy Study) a las 12 semanas (figura 1).

Al comparar los resultados a 12 semanas y 12 meses se observa que los tres grupos presentaron tendencia a mantenerse sin cambios importantes. El grupo con < 5 letras de mejoría después de tres inyecciones no presentó mejoría adicional al seguir inyectándose a 12 meses. Con estos resultados, Dugel ${ }^{1}$ afirma que «si los objetivos de mejoría de agudeza visual no se obtienen después de tres inyecciones consecutivas de anti-VEGF, no se justifican inyecciones adicionales para mejorar la agudeza visual y se sugiere cambiar de tratamiento".

Las respuestas de estos tres grupos se analizaron a los 12 meses y se pueden observar en la figura 2, que muestra las tres curvas. El análisis multivariado entre los tres grupos entre la semana 12 y el mes 12 muestra significancia estadística $(p<0.001)^{1}$.
En conclusión, estos resultados, que se obtuvieron utilizando solo un anti-VEGF, indican que el $40 \%$ de los pacientes con EMD no son respondedores a esta terapia. Este alto porcentaje de no respondedores justifica el uso de otras modalidades terapéuticas, principalmente tratamientos con diferente mecanismo de acción, como son los esteroides.

La respuesta a la terapia anti-VEGF en el EMD debe ser cuidadosamente valorada a las 12 semanas (después de tres inyecciones mensuales consecutivas). Si la respuesta no muestra mejoría de $>5$ letras en esta evaluación, se sugiere no continuar con antiangiogénicos y valorar el cambio a terapia con esteroides (dexametasona) $)^{1-4}$.

\section{Bibliografía}

1. Dugel $P$. Can long-term response to anti-VEGF therapy be predicted after three injections in patients with DME (EARLY)? A post hoc analysis of the ranibizumab arms from Protocol I point to "yes". 2015 AAO Meeting. November 2015, Las Vegas, Nevada, United States.

2. Jampol L, Glassman A, Bressler N. Comparative effectiveness trial for diabetic macular edema: three comparisons for the price of 1 study from the Diabetic Retinopathy Clinical Research Network. JAMA Ophthalmol. 2015;133:983-4

3. Nguyen Q, Brown D, Marcus D, Boyer D, Patel S, Feiner L, et al. Ranibizumab for diabetic macular edema: results from 2-phase III randomized trials: RISE and RIDE. Ophthalmology. 2012;119:789-801.

4. Elman M, Aiello L, Beck R, Bressler N, Bressler S, Edwards A, et al. Randomized trial of ranibizumab plus prompted or deferred laser or triamcinolone plus prompted or deferred laser to treat diabetic macular edema. Ophthalmology. 2010;117:1064-77. 


\title{
Esteroides en el edema macular diabético: triamcinolona, fluocinolona y dexametasona. Ventajas del uso de dexametasona como tratamiento de primera línea
}

\author{
Gabriela Lopezcarasa-Hernández* \\ Hospital Ángeles Lomas, Instituto de Ciencias Oftalmológicas, Huixquilucan, Estado de México, México
}

Existe amplia evidencia que indica que actualmente el tratamiento de primera línea para el edema macular diabético (EMD) es la terapia antiangiogénica mediante la aplicación intravítrea de inhibidores del factor de crecimiento vascular endotelial (anti-VEGF [Vascular Endothelial Growth Factor]). Aun así, dos tercios de los pacientes presentan EMD persistente después de 6 meses de tratamiento ${ }^{1,2}$.

El uso de esteroides, como la triamcinolona, la fluocinolona y la dexametasona, ha demostrado también su utilidad en el tratamiento del EMD. Es indispensable saber que los glucocorticoides son complejos hormona-receptor con gran potencial antiinflamatorio e inmunosupresor, que ejercen numerosos beneficios en las enfermedades inflamatorias. Los glucocorticoides son hormonas lipófilas con una estructura hidrófoba que fácilmente atraviesan la membrana celular. Son hormonas naturales, las cuales se producen en la corteza adrenal en relación de 5 a $30 \mathrm{mg}$ de cortisol activo por día, que interviene en múltiples funciones del organismo.

Brevemente debemos recordar que los glucocorticoides ejercen una función antiinflamatoria e inmunosupresora, afectando principalmente a los leucocitos; disminuyen el número de monocitos/macrófagos y afectan la síntesis de citocinas y prostaglandinas (como las interleucinas 1,2 y 6 , el factor de necrosis tumoral alfa y el interferón), reduciendo o inhibiendo su número. En referencia a los granulocitos, disminuyen el número de eosinófilos y basófilos al mismo tiempo que aumenta el de neutrófilos circulantes. También reducen la adhesión de las moléculas a las células endoteliales y la proliferación de fibroblastos, reduciendo la permeabilidad vascular.

La influencia de los glucocorticoides en todas las células inmunitarias, tanto primarias como secundarias, en los tejidos y órganos, así como la supresión, la disminución de la transcripción y la acción que ejercen en los factores humorales que intervienen en el proceso inflamatorio, les confieren un poder terapéutico excepcional. Los glucocorticoides sintéticos inhiben las citocinas proinflamatorias e influyen en otros mecanismos con efecto vascular y activación del drenaje, favoreciendo su efecto antiedematoso aunado al efecto parcial que ejercen en la supresión parcial del $\mathrm{VEGF}^{3,4}$.

El modo de acción de los glucocorticoides depende de las dosis; aquellas dosis más altas activan los efectos no genómicos (figuras 1 a 4$)^{4}$.

Las tres propiedades que se han adjudicado a los glucocorticoides con efecto sobre el EMD son ${ }^{5-7}$ :

- Efectos antiinflamatorios, mediante la represión principalmente del factor de necrosis tumoral alfa, la interleucina 6 y el factor nuclear kappa B que inhibe la enzima óxido nítrico sintetasa.

- Efectos vasculares, al reprimir las citocinas que interfieren en el deterioro de la barrera hematorretiniana, favoreciendo su restauración. 


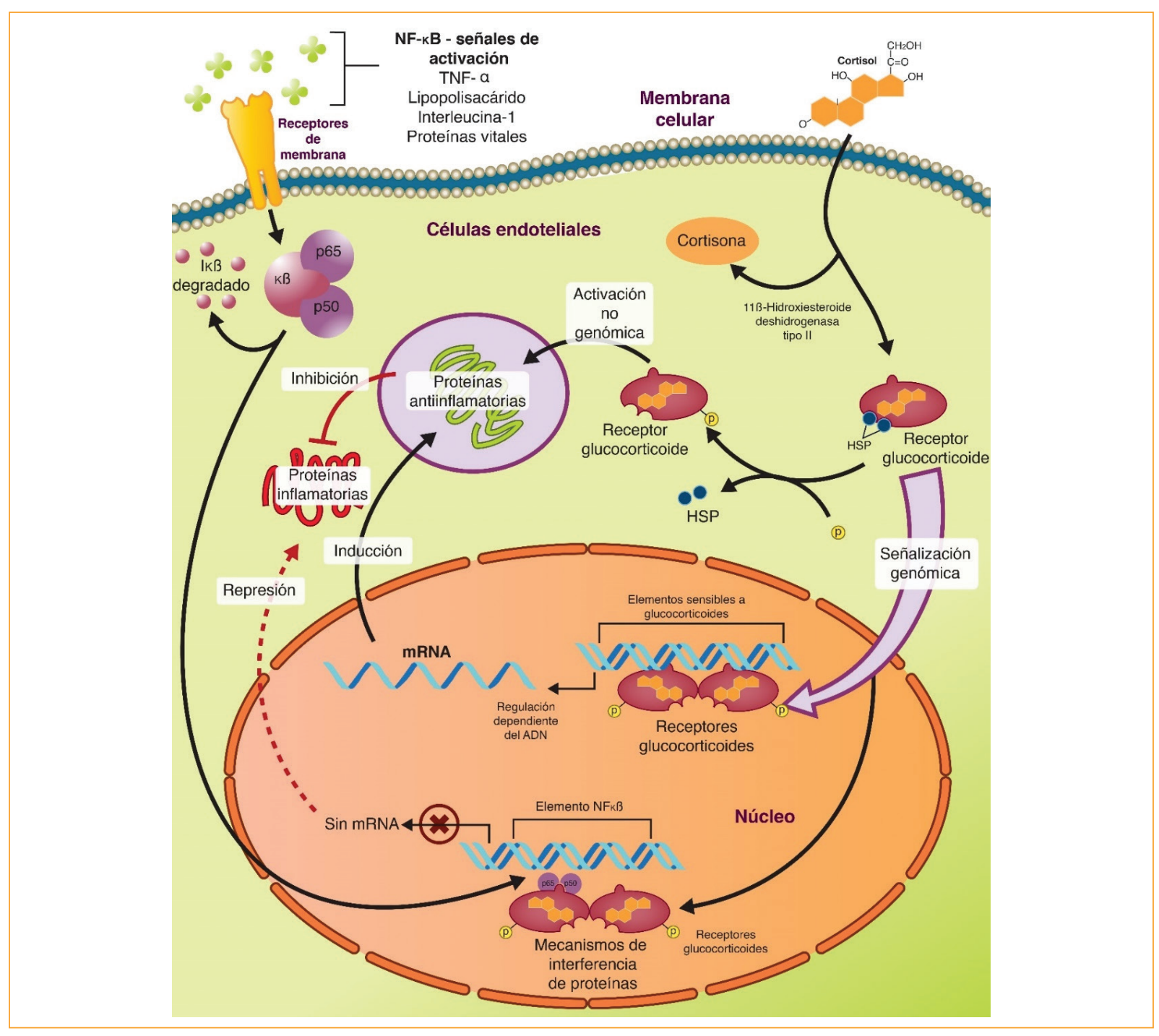

Figura 1. Mecanismos de acción directo e indirecto que se consideran genómico y epigenético: no genómico.

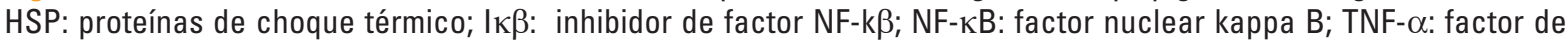
necrosis tumoral alfa.

- Efectos sobre las células gliales retinianas, mediante la reducción de la inflamación de las células de Müller que desempeñan un papel hidroiónico activo a través de los canales de potasio y acuaporina, restaurando la distribución de los canales de potasio y agua que infieren en el mecanismo antiedematoso con una función de drenaje.

Los efectos adversos más importantes de los glucocorticoides se observan al utilizarlos en dosis altas y por largos periodos de tiempo, estos incluyen alteración del metabolismo de los carbohidratos, de los ácidos grasos, de las proteínas, así como desmineralización ósea. Estos efectos adversos generalmente no son de trascendencia con su uso en oftalmología, pues las dosis no son altas y los medios de administración son directos en el globo ocular ${ }^{8}$.

Entre los agentes esteroideos sintéticos o glucocorticoides (figura 5) se encuentran la prednisolona, la metilprednisolona, la triamcinolona, la fluocinolona y la dexametasona, que tienen más efecto antiinflamatorio que actividad mineralocorticoide en relación al cortisol, lo cual optimiza los resultados que se buscan en el tratamiento farmacológico de las diferentes patologías ${ }^{4}$.

Es muy importante hacer mención a los cambios que se han realizado en estos glucocorticoides, como en los grupos acetónido que confieren poder antiinflamatorio muy potente o la fluoración de la dexametasona que aumenta la unión del receptor glucocorticoide y la 


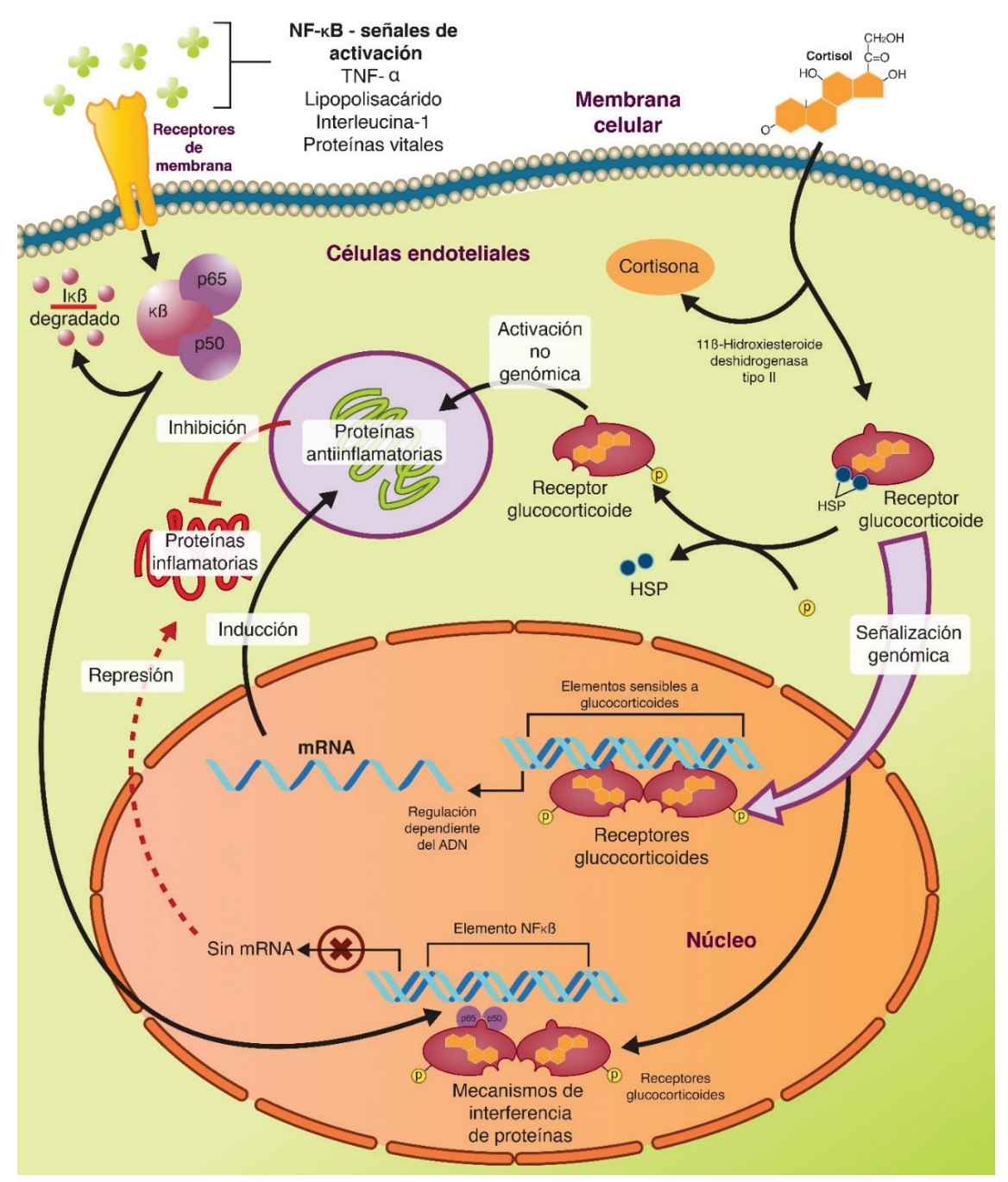

Figura 2. El mecanismo directo genómico provoca la síntesis de proteínas antiinflamatorias, ya sea por transcripción 0 por transducción. HSP: proteínas de choque térmico; Iк $\beta$ : inhibidor de factor NF-k $\beta$; NF-kB: factor nuclear kappa B; TNF- $\alpha$ : factor de necrosis tumoral alfa.

actividad antiinflamatoria, o el grupo fosfato que mejora la solubilidad en agua y tiene importantes repercusiones en la reducción de algunos efectos secundarios en el trabéculo $0^{4}$.

Toda la evolución actual en relación con la optimización del riesgo/beneficio en la administración localizada o la liberación y administración paulatina de los esteroides ha resultado en beneficios para los pacientes al regular las características farmacodinámicas de estos fármacos. Unas de las mayores desventajas de la terapia antiangiogénica para el tratamiento del EMD son la frecuencia, el número de inyecciones y el apego al tratamiento por parte del paciente que se requiere para controlar el edema. Además, el riesgo acumulado de endoftalmitis por aplicaciones constantes se eleva hasta un $1 \%$ posterior a 40 inyecciones ${ }^{9,10}$.

Respecto a las opciones de tratamiento, es importante considerar la fisiopatología del EMD, que involucra diferentes factores de crecimiento y citocinas, tales como interleucinas, monocitos, factor de necrosis tumoral y VEGF principalmente, generando cambios vasculares, leucostasis con la consecuente alteración de la adhesión intracelular (ICAM-1), diapédesis, hiperpermeabilidad y liberación de proteínas inflamatorias. Todas estas alteraciones generan daño endotelial, engrosamiento de la membrana capilar basal, acúmulo de componentes extracelulares y daño a la microvasculatura retiniana con lesión de la barrera 


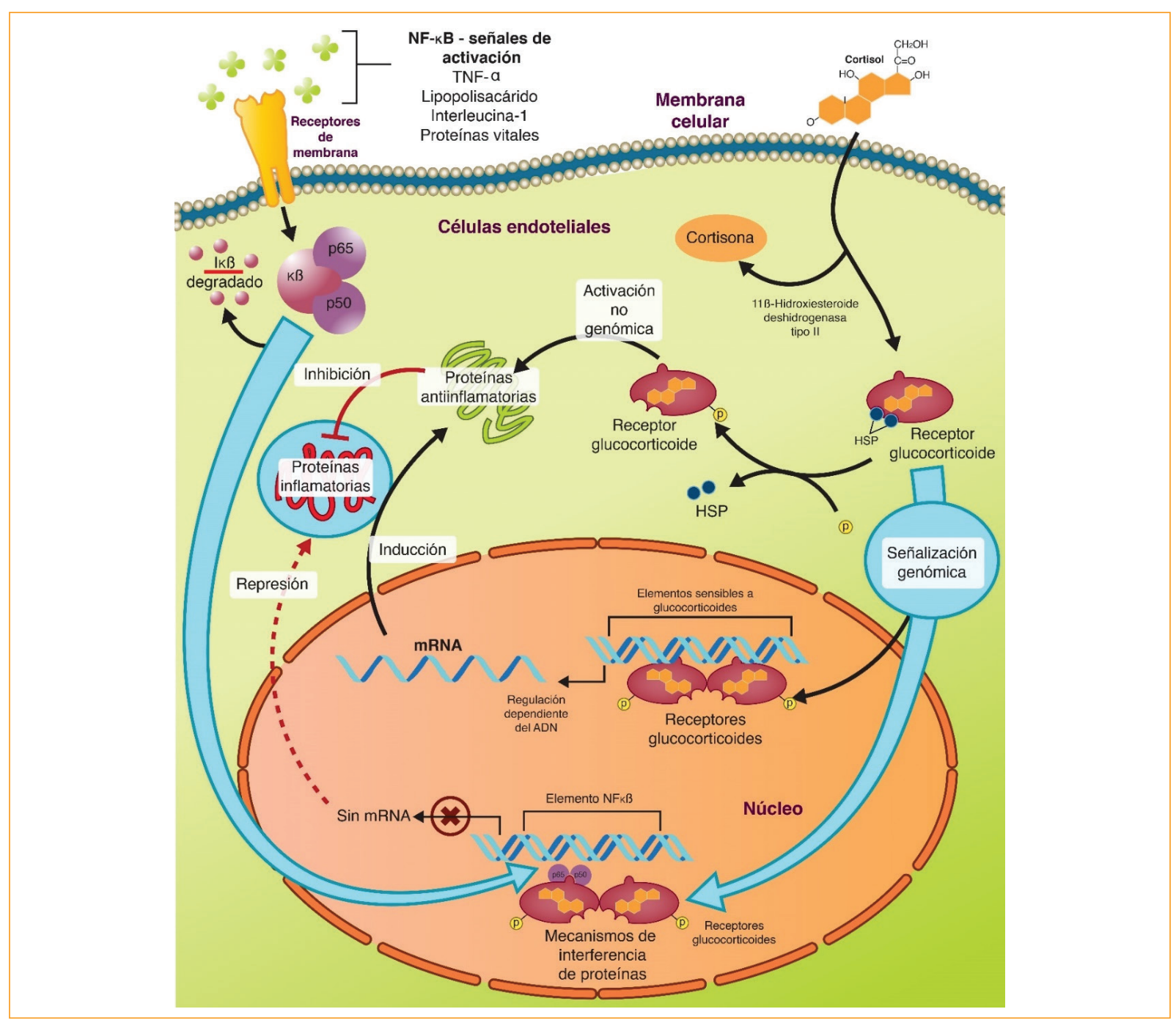

Figura 3. El mecanismo indirecto genómico inhibe el modo de citocinas proinflamatorias. HSP: proteínas de choque térmico; Iк $\beta$ : inhibidor de factor NF-k $\beta$; NF- $\kappa B$ : factor nuclear kappa B; TNF- $\alpha$ : factor de necrosis tumoral alfa.

hematorretiniana y el consecuente acúmulo de líquido en el área macular.

Lo anterior justifica el uso de los esteroides como una terapia de abordaje que resulta multifuncional, ya que implementa varios mecanismos de acción, como son la disminución de los factores de inflamación y la injerencia en la síntesis de VEGF.

La aplicación de tratamientos intravítreos con glucocorticoides ha demostrado una disminución importante del proceso inflamatorio con acción en la barrera hematorretiniana a través de la inhibición de ICAM-111-13.

En relación con el desarrollo de hipertensión ocular y catarata relacionados con los esteroides intravítreos, debemos saber que está relacionado con la solubilidad que estos tienen en agua y su afinidad por la malla trabecular; una mayor solubilidad también significa que el medicamento se elimina más rápidamente. El acetónido de triamcinolona es lipófilo y la dexametasona es hidrosoluble, motivo por el cual requiere un sistema de liberación lenta que aumente su sobrevida, pero a la vez esto favorece que sus efectos adversos sean menores que los de los agentes lipófilos, como la triamcinolona y la fluocinolona ${ }^{14,15}$.

Las opciones alternativas al tratamiento anti-VEGF intraocular son los esteroides sintéticos, y se han utilizado el acetónido de triamcinolona, los implantes de dexametasona y los implantes de acetónido de fluocinolona. 


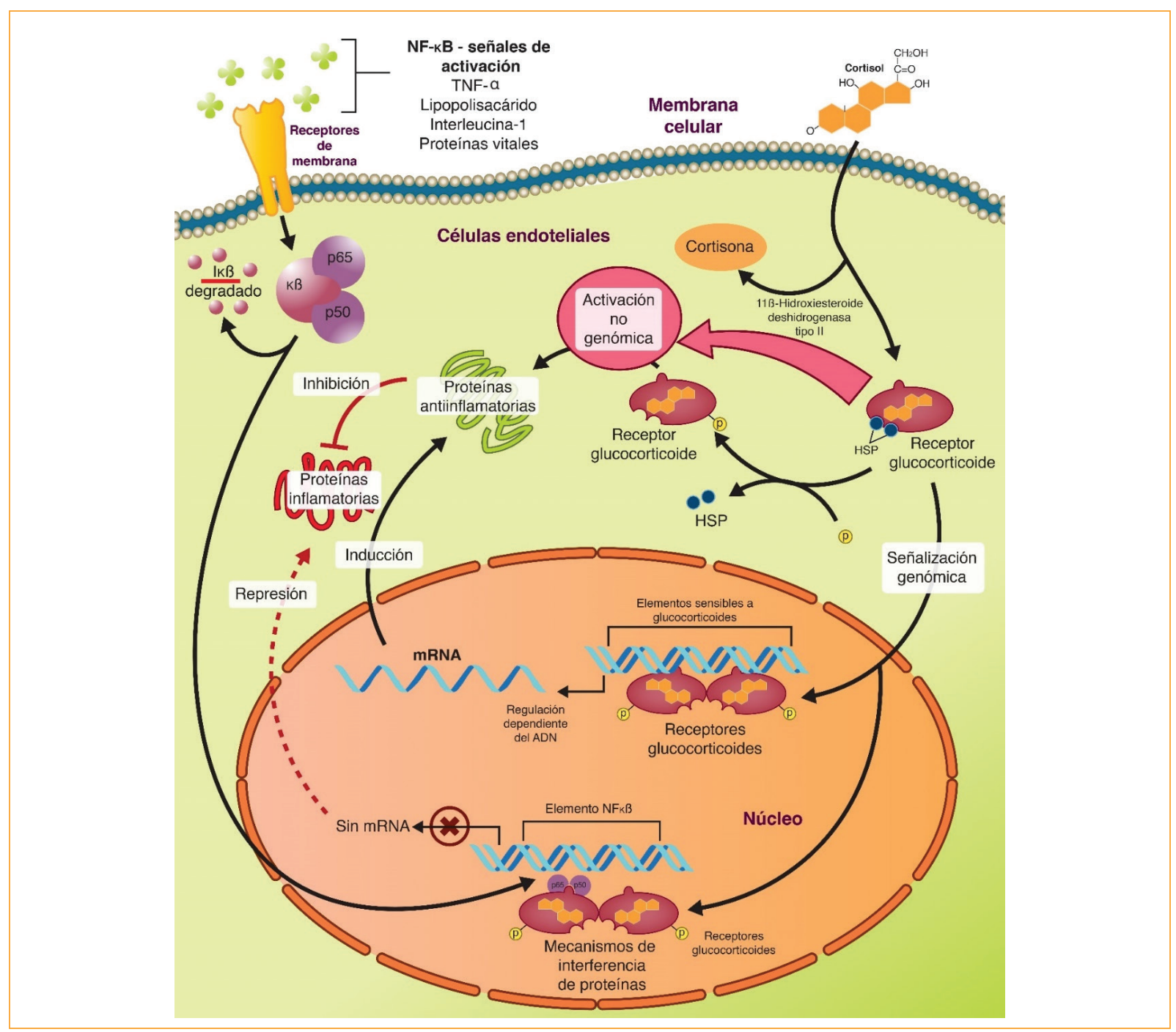

Figura 4. Mecanismo epigenético no genómico. Actúa a través de la activación rápida de proteínas antiinflamatorias

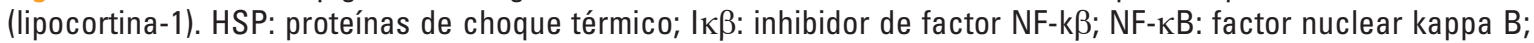
TNF- $\alpha$ : factor de necrosis tumoral alfa.

\section{Esteroides intravítreos}

\section{Acetónido de triamcinolona}

Jonas y Söfker ${ }^{16}$ fueron los primeros en inyectar acetónido de triamcinolona en la cavidad vítrea para el EMD, en el año 2000, demostrando su regresión y obteniendo una ganancia visual por un periodo de hasta 5 meses. Posterior a esto se popularizó su uso a razón de $4 \mathrm{mg}$ como dosis intravítrea, decisión basada en que la presentación comercial de este medicamento era de $40 \mathrm{mg} / \mathrm{ml}$.

Existen publicaciones que reportan que el acetónido de triamcinolona tiene 7.5 más potencia que el cortisol, siendo su vida media en ojos no vitrectomizados de 18.6 días, y de 3.2 días en ojos vitrectomizados. Así mismo, se conoce que una inyección de $4 \mathrm{mg}$ dura aproximadamente 3 meses en un ojo humano no vitrectomizado ${ }^{11}$.

El acetónido de triamcinolona es lipófilo y eso le hace más afín al cristalino y a la red trabecular (generando efectos adversos). Su solubilidad en agua es mucho menor que la del fosfato de dexametasona. La triamcinolona reduce los valores de citocinas y quimiocinas, así como también del VEGF, pero tal vez a valores casi normales. Teniendo en cuenta el conocimiento obtenido sobre las funciones fisiológicas importantes del 


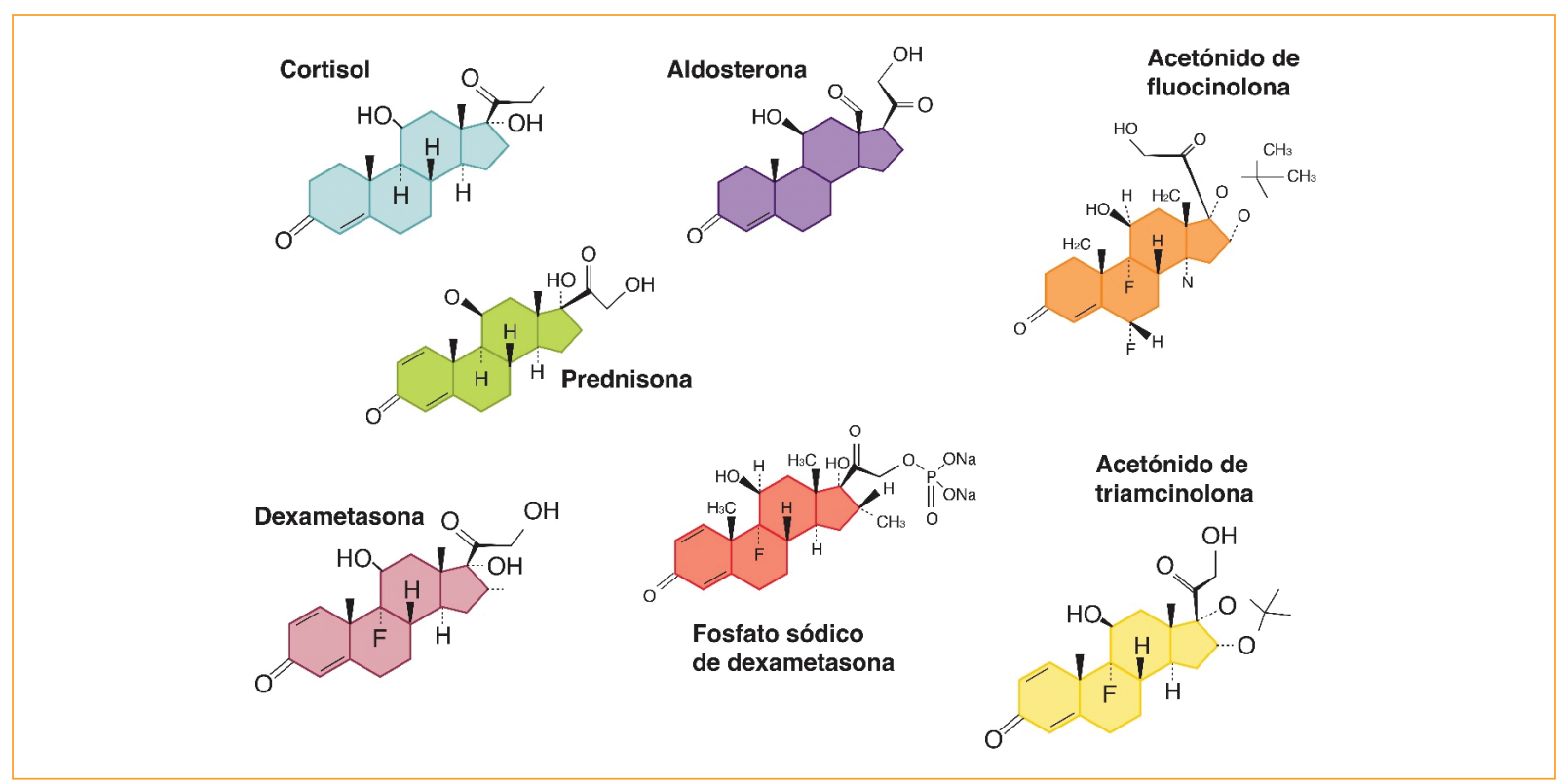

Figura 5. Estructuras de los glucocorticoides sintéticos.

VEGF, se plantea el interrogante de si es útil neutralizarlo por completo ${ }^{11,14,15,17}$.

Se realizaron estudios para valorar diferentes dosis-respuesta en ojos con EMD y se observó que sí había una relación dependiente de la dosis, aunque también una elevación de la presión intraocular paralela relacionada con el aumento de la dosis ${ }^{18}$.

El acetónido de triamcinolona libre de preservadores se microniza y se liofiliza con un tamaño de partícula más uniforme, a diferencia de la inyección de $40 \mathrm{mg}$ $\left(\right.$ Kenalog $\left.{ }^{\circledR}\right)$, que se fabrica con una metodología más antigua y tiene diferentes tamaños de partículas, por lo cual se depura más lentamente en el vítreo.

El Protocolo $B$ es un estudio prioritario en la evaluación del acetónido de triamcinolona en el EMD, en el que se comparó el láser focal con $104 \mathrm{mg}$ de acetónido de triamcinolona intravítreo, con retratamientos a intervalos de 4 meses. En el punto de corte de 4 meses, los pacientes con $4 \mathrm{mg}$ tenían mejor campo visual computarizado (CVC) que los de los otros dos grupos, lo cual para el año ya no resultaba estadísticamente significativo en los tres grupos. Sin embargo, a los 2 años se observó mejor agudeza visual corregida (AVC) en el grupo de láser en comparación con el de acetónido de triamcinoIona, esto secundario aparentemente al desarrollo de catarata. Un dato de mucha importancia es el desarrollo de presión intraocular elevada $\geq 10 \mathrm{mmHg}$ de la basal en ambos grupos de acetónido de triamcinolona: $40 \%$ en el grupo de $4 \mathrm{mg}, 20 \%$ en el grupo de $1 \mathrm{mg}$ y $10 \%$ en el grupo de láser. Los pacientes con presión intraocular $>30 \mathrm{mmHg}$ recibieron tratamiento tópico y 4 (23\%) tuvieron que ser sometidos a cirugía de glaucoma ${ }^{13}$.

Otro estudio trascendente fue el Protocolo I de DRCR.net, a 5 años, que comparó placebo con láser, $0.5 \mathrm{mg}$ de ranibizumab más láser, $0.5 \mathrm{mg}$ ranibizumab más láser diferido (> 24 semanas) y $4 \mathrm{mg}$ de acetónido de triamcinolona más láser. A 1 año, el grupo de acetónido de triamcinolona y láser resultó en una ganancia de 4 letras respecto a la agudeza visual basal, comparado con 3 letras en el grupo de láser y 9 letras en ambos grupos de ranibizumab y láser.

Como efectos adversos se reportó un aumento $\geq$ $10 \mathrm{mmHg}$ por arriba de la presión intraocular basal en el $50 \%$ de los pacientes con acetónido de triamcinoIona, frente al $9 \%$ con ranibizumab y el $11 \%$ con láser. Así mismo, el grupo de acetónido de triamcinolona requirió cirugía de catarata en el $59 \%$ de los casos, frente al $14 \%$ en los grupos de ranibizumab y de láser a los 2 años de seguimiento. En este estudio se concluye que los resultados son similares en todos los grupos, pero con mayores efectos relacionados al grupo de acetónido de triamcinolona ${ }^{13,19}$.

En el análisis post hoc realizado a este mismo Protocolo I se reporta que un tercio de los pacientes experimentaron edema persistente durante el primer año, con un grosor central de la retina $\geq 250 \mu \mathrm{m}$ 
posterior al inicio de ranibizumab, el cual continuó para la mayoría de los pacientes hasta el segundo año $0^{17,20}$.

Al ajustar por los posibles factores de confusión, incluida la mejor agudeza visual corregida (AVMC) inicial, se mantuvo una asociación negativa significativa entre el edema persistente y el aumento de la AVMC a largo plazo entre las semanas 52, 104 y $156^{20}$.

Este estudio post hoc llevó a una conclusión muy importante: en los pacientes con edema persistente 0 mayor tiempo sin tratamiento, la agudeza visual final es significativamente menor en aquellos con mayor duración del edema, estimando que la mejoría de la visión fue en promedio de 5 letras menos en los pacientes con mayor duración del edema. Esto nos lleva a la idea de que la consideración temprana de estrategias adicionales de tratamiento o switch es imperativa para maximizar el beneficio potencial de la visión, evitando el daño neuronal irreversible ${ }^{20}$.

Además del aumento de la presión intraocular y la mayor incidencia de catarata antes mencionados, existen publicaciones sobre endoftalmitis no infecciosa relacionada con el conservador utilizado, específicamente en Kenalog ${ }^{\circledR} 40 \mathrm{mg} / \mathrm{ml}$ en uso intraocular, y sobre pseudoendoftalmitis secundaria al depósito de cristales en la cámara anterior, aunado al hecho de que su uso es fuera de indicación, ya que no está aprobado por la Food and Drug Administration (FDA) para este tipo de tratamientos ${ }^{21,22}$.

Existen dos presentaciones de acetónido de triamcinolona sin conservador, Triesence ${ }^{\circledR} 40 \mathrm{mg} / \mathrm{ml}$ y Trivaris ${ }^{\circledR} 80 \mathrm{mg} / \mathrm{ml}$, que ya no presentan este tipo de reacciones inflamatorias secundarias, pues se fabrican $\sin$ alcohol bencílico polisorbato 80 , que era el conservador antes relacionado con este tipo de efectos adversos ${ }^{19,20}$.

Wykoff, et al. ${ }^{23}$ han reportado el uso de acetónido de triamcinolona en inyección supracoroidea en un intento de alcanzar concentraciones terapéuticas en la retina y al mismo tiempo disminuir los efectos adversos en la cámara anterior, observando una buena tolerabilidad.

Se describe el protocolo HULK, en el que se estudia la administración de acetónido de triamcinolona sin conservadores solo o en combinación con aflibercept intravítreo para el tratamiento del EMD.

Se administraron $4 \mathrm{mg}$ en $0.1 \mathrm{ml}$ por vía supracoroidea, 4-4.5 mm posterior al limbo, con agujas de $900 \mathrm{a}$ $1100 \mu \mathrm{m}$ usando el microinyector en todos los pacientes, y a aquellos sin tratamiento previo se les agregó una dosis de aflibercept $(0.05 \mathrm{ml}, 2.0 \mathrm{mg})$, en un total de 20 pacientes. A partir del segundo a quinto meses, los pacientes fueron tratados por razón necesaria si cumplían con los criterios de retratamiento descritos. Con esta técnica, en ambos grupos del estudio se demostraron una ganancia de letras y beneficios anatómicos basados en el grosor central macular. A los 6 meses, el $89 \%$ de los pacientes en el grupo combinado presentaban un $50 \%$ de reducción del grosor macular. El promedio de aplicaciones fue de 2.6 en los pacientes sin tratamiento previo y de 3.3 en aquellos con tratamiento previo en un periodo de 6 meses.

Se reportaron efectos adversos relacionados con la técnica de aplicación. El 10\% de los pacientes reportaron elevación de la presión intraocular y hubo tres casos de progresión de catarata. El estudio HULK es el primero realizado con esta técnica en monoterapia como tratamiento de las enfermedades vasculares de la retina. Este estudio demostró que el acetónido de triamcinolona libre de conservadores resulta efectivo y seguro para la población con $\mathrm{EMD}^{23}$.

\section{Implantes de acetónido de fluocinolona (Iluvien ${ }^{\circledR}$ y Retisert $^{\oplus}$ )}

El acetónido de fluocinolona tiene una potencia antiinflamatoria similar a la de la dexametasona. Existen dos presentaciones en el mercado. El primer implante no biodegradable disponible en el mercado que contiene acetónido de fluocinolona es lluvien ${ }^{\circledR} 0.19 \mathrm{mg}$, que se inyecta en la cavidad vítrea con una aguja de calibre 25. Está diseñado para liberar $0.20 \mu \mathrm{g}$ lentamente hasta por 3 años 24,25 .

El segundo implante disponible es Retisert ${ }^{\circledR}$, un implante no biodegradable que contiene $0.59 \mathrm{mg}$ de acetónido de fluocinolona, el cual se sutura a la pars plana y está diseñado para liberar $0.59 \mu \mathrm{g}$ al día por un mes y posteriormente 0.3-0.4 $\mu \mathrm{g}$ al día por un periodo de hasta 30 meses $^{24,26,27}$.

El cambio a lluvien ${ }^{\circledR}$ en el EMD se reportó como benéfico, realizando un cambio de tratamiento cada 2.9 meses con tratamiento anti-VEGF a 14.3 meses con Iluvien ${ }^{\circledR}$. Este medicamento fue autorizado por la FDA en 2014 para el tratamiento del EMD crónico sin antecedentes de glaucoma, en dosis de $0.19 \mathrm{mg}$. Los estudios de farmacocinética muestran una liberación sostenida dentro del ojo por un periodo de $1 \mathrm{año}^{13}$.

El estudio FAME estudió el EMD refractario al tratamiento con láser micropulsado, dividiendo 956 pacientes en tres grupos en los que se utilizaron $0.2 \mathrm{mg}$ de Iluvien ${ }^{\circledR}$ por día, 0.5 mg de lluvien ${ }^{\circledR}$ por día o placebo, con terapia de rescate a 6 meses con láser micropulsado. La duración promedio del edema en estos pacientes era de 5.1 años, por lo cual la definición 
operativa de EMD crónico fue aquél con más de 3 años de evolución. La respuesta fue favorable al tratamiento con lluvien ${ }^{\circledR}$ a 3 años, reportando una ganancia $>5$ letras en el $28.7 \%$, el $27.8 \%$ y el $18.9 \%$, respectivamente. El grupo con menor dosis de lluvien ${ }^{\circledR}$ requirió terapia de rescate con láser micropulsado en el $40 \%$ de los casos. El estudio de un subgrupo demostró que los pacientes con mayor cronicidad, referida como más de 3 años de evolución, tuvieron mejor respuesta a lluvien ${ }^{\circledR}$ que aquellos con menor tiempo de evolución ${ }^{13,27,28}$.

En un análisis post hoc en el que se comparó en EMD crónico (más de 3 años) y EMD agudo el porcentaje de pacientes que ganaron $\geq 15$ letras, este fue significativamente mayor en el grupo de pacientes crónicos, del $34 \%$ frente al $13.4 \%$ en el grupo placebo, comparados con los del grupo de EMD agudo, que fue del $22.3 \%$ frente al $27.8 \%$ en el grupo placebo, lo que sugiere que se obtiene mayor beneficio en los pacientes crónicos ${ }^{13,27,28}$.

El porcentaje de pacientes diagnosticados con glaucoma que requirieron cirugía fue alto en ambos grupos de tratamiento: 7.6 y $3.7 \%$ en los grupos de 0.5 y $0.2 \mu \mathrm{g}$, respectivamente, frente a $0.5 \%$ en el grupo placebo. Esto indica una relación importante en el desarrollo de glaucoma en estos pacientes, al igual que en el desarrollo de catarata, que fue del $50.9 \%$ y el $41.1 \%$ con las dosis respectivas, frente a solo el $7 \%$ en el grupo con placebo ${ }^{13,25}$.

Iluvien ${ }^{\circledR}$ fue autorizado en Europa para el tratamiento del EMD persistente o recurrente a pesar de haber recibido tratamiento previo. Existen varios estudios europeos de la vida real en los que se confirma la respuesta benéfica, tanto anatómica como funcional, con este implante en el EMD de pacientes con mala respuesta a la terapia antiangiogénica o a otros corticosteroides, lo cual marca una diferencia importante con el estudio FAME, que incluyó no respondedores al láser micropulsado ${ }^{29}$.

En un estudio realizado en el Reino Unido se incluyó un $75 \%$ de pacientes con respuesta insuficiente a la terapia antiangiogénica. De estos, $20 \%$ tuvieron una ganancia de 15 o más letras y el promedio desde la basal, fue una ganancia de 5.1 letras. Del $18 \%$ de estos pacientes que corrigieron 20/40 en el inicio se aumentó la proporción al $40 \%$ de los pacientes a los 24 meses del tratamiento. Se reportó que se duplicó el porcentaje de pacientes que corrigieron $\geq 20 / 40(40 \%)$ desde los 12 meses $^{30}$.

Los estudios de costo-efectividad realizados en Europa concluyeron que lluvien ${ }^{\circledR}$ es una opción adecuada para pacientes con EMD crónico, otorgando un valor adecuado particularmente en aquellos pseudofáquicos por el riesgo de formación de catarata reportado previamente con este medicamento ${ }^{31}$.

En cuanto al implante de acetónido de fluocinolona no biodegradable Retisert ${ }^{\circledR}$, está indicado principalmente para el tratamiento de uveítis posteriores no infecciosas ${ }^{24,26}$. Sin embargo, su uso fuera de indicación $(0.59 \mathrm{mg})$ se reportó benéfico en los ojos con EMD recurrente o persistente a pesar de la aplicación de láser micropulsado. Se hizo un estudio multicéntrico en el que se comparó el uso de Retisert ${ }^{\circledR}$ frente al tratamiento habitual para el EMD, y se halló una mejoría de la AVMC a los 2 años del implante en comparación con el grupo de tratamiento habitual. A 3 años del estudio ya no había diferencias significativas, lo que coincide con la descripción de que el medicamento del implante se había consumido en su totalidad ${ }^{32}$.

\section{Implante de dexametasona (Ozurdex $\left.{ }^{\oplus}\right)$}

La dexametasona tiene una potencia 10 veces mayor para la transactivación del receptor de glucocorticoide que para el de mineralocorticoide. Muestra 25 veces más poder antiinflamatorio que el cortisol, potencia que se determina por su efecto vasoconstrictor, motivo por el cual se considera uno de los corticoides más potentes. Es importante recordar que suprime múltiples mediadores inflamatorios, incluyendo el VEGF. Su actividad sobre los principales mediadores de la inflamación se muestra en la figura 68,32. Además, la dexametasona es hidrosoluble, lo que hace que su vida media en el ojo humano sea de 5.5 horas.

Se ha desarrollado un implante biodegradable de dexametasona de liberación prolongada (Ozurdex ${ }^{\circledR}$ ) que contiene $700 \mu \mathrm{g}$ en un polímero que se degrada por completo de forma paulatina en agua y dióxido de carbono una vez insertado en la cavidad vítrea. La característica hidrófoba de los glucocorticoides fue modificada en este implante intraocular, volviéndolo hidrosoluble y generando así una disminución de algunos efectos adversos relacionados con la triamcinoIona, que se depositaba en el trabéculo (glaucoma) ${ }^{11}$.

El implante de Ozurdex ${ }^{\circledR} 0.7 \mathrm{mg}$ fue aprobado por la FDA para el tratamiento de pacientes con EMD en septiembre de 2014, y por la Agencia Europea de Medicamentos en julio de 2014 con la indicación para pacientes con EMD pseudofáquico o aquellos considerados no respondedores a un tratamiento previo o que tuvieran contraindicación para recibir terapia antiVEGF. Se describió que el implante libera medicamento por un periodo promedio de 6 meses, que fue 


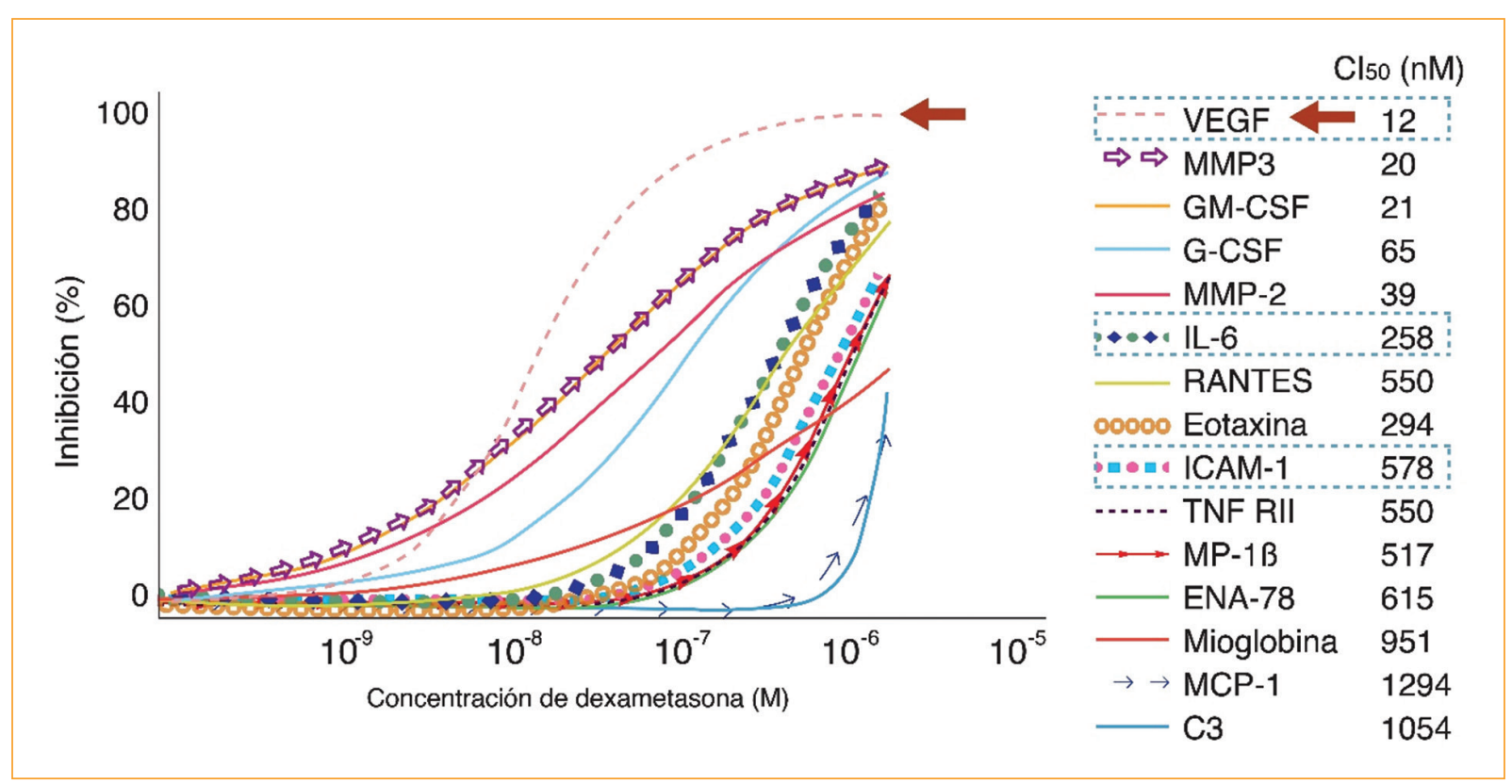

Figura 6. Mediadores de la inflamación sobre los que tiene actividad la dexametasona para controlar la cascada de la reacción inflamatoria.

modificado en los estudios de la vida real al observar una duración promedio de 2.3 a 4.9 meses $^{13}$.

Existe un gran número de protocolos desarrollados en relación con el uso de dexametasona intravítrea para el tratamiento del EMD, de los cuales mencionaremos los más importantes.

El primer estudio que evaluó el uso de dexametasona para el EMD fue el PLACID, un estudio multicéntrico, aleatorizado y controlado, que comparó el implante Ozurdex ${ }^{\circledR} 0.7 \mathrm{mg}$, el láser micropulsado al primer mes y la monoterapia con láser e inyección de placebo. Los 253 pacientes que integraron el estudio podían recibir tres tratamientos más de láser y un segundo implante de Ozurdex $^{\circledR}$ si era necesario. A pesar de que en los resultados a 1 año no hubo diferencias estadísticamente significativas entre los grupos, sí se reportó una ganancia de al menos 10 letras en los grupos, siendo mayor en el grupo de Ozurdex ${ }^{\circledR}$ con láser (31.7\%) que en el de monoterapia con láser (11\%). El incremento de la presión intraocular fue más frecuente en el grupo de Ozurdex ${ }^{\circledR}$, en el que se observó que el $16 \%$ de los pacientes tuvieron un aumento $\geq 10 \mathrm{mmHg}$ de la basal, el $16.8 \% \geq 25 \mathrm{mmHg}$ y el $4 \% \geq 35 \mathrm{mmHg}$, siendo importante señalar que ningún paciente fue sometido a cirugía filtrante ${ }^{13,33}$.

El estudio MEAD, aleatorizado, controlado y multicéntrico, es considerado el más importante realizado con dexametasona, pues se estudiaron dos dosis diferentes (0.7 y $0.35 \mathrm{mg}$ ) frente a placebo en 1048 participantes con EMD, AVMC de 20/50 a 20/200 y grosor macular central $(G M C) \geq 300 \mu \mathrm{m}$. El retratamiento se permitía en aquellos pacientes que cumplían con los criterios preestablecidos, y debía ser aplicado cada 6 meses como mínimo. El $67 \%$ de estos pacientes completaron el estudio a 3 años ${ }^{34}$.

El número promedio de implantes recibido fue de cinco y la mayor ganancia en letras la obtuvo el grupo de $0.7 \mathrm{mg}: 8$ letras frente a 2 en el grupo con placebo. Al final del tercer año, los porcentajes de pacientes con una ganancia $\geq 15$ letras fueron del $22.2 \%$ y el $18.4 \%$ en los grupos de Ozurdex ${ }^{\circledR}$ de 0.7 y 0.35 mg, respectivamente, frente al $12 \%$ en el grupo con placebo. En este mismo estudio se demostró una reducción de la AVMC en promedio a los 15 meses del inicio del tratamiento, atribuida a la presencia de catarata, con una subsecuente recuperación a los 3 años, lo cual se explica por la realización de cirugía, observándose que los pacientes regresaban a la ganancia de letras que habían obtenido al inicio de sus tratamientos (figura 7$)^{34}$.

El estudio MEAD reportó un incremento en la presión intraocular $\geq 10 \mathrm{mmHg}$ en el $27.7 \%$ y el $24.8 \%$ de los pacientes de los grupos de 0.7 y $0.35 \mathrm{mg}$, respectivamente, frente al $13 \%$ en el grupo con placebo; el aumento $\geq 35 \mathrm{mmHg}$ ocurrió en el $6.6 \%$ y el $5.2 \%$ con las dosis respectivas, frente al $0.9 \%$ con placebo. El 


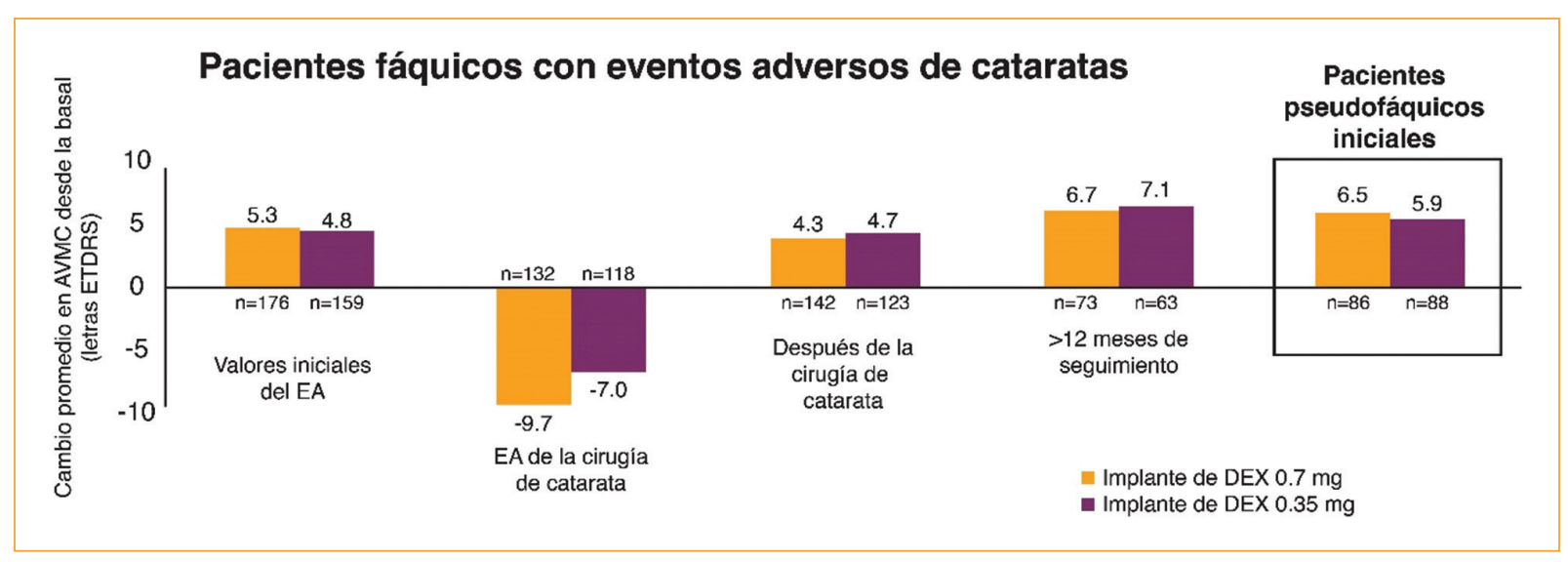

Figura 7. Los pacientes que se sometieron a cirugía de catarata finalmente obtuvieron el mismo resultado que los pacientes pseudofáquicos. AVMC: agudeza visual mejor corregida; DEX: dexametasona; EA: evento adverso; ETDRS: Early Treatment Diabetic Retinopathy Study.

$41.5 \%$ y el $37.6 \%$ de los pacientes con Ozurdex ${ }^{\circledR}$ de 0.7 y $0.35 \mathrm{mg}$, respectivamente, o un tercio de los pacientes estudiados, requirieron medicamento para controlar la presión intraocular, reportando finalmente que solo el $0.3 \%$ de ellos requirieron cirugía filtrante ${ }^{34}$.

Un análisis post hoc de los datos del Protocolo I de DRCR.net demostró que la respuesta de la AVMC a las 12 semanas predice la respuesta a 3 años, ya que la mayoría de los pacientes con una ganancia $<5$ letras a las 12 semanas no mostraron mejoría en el seguimiento a 3 años con ranibizumab más láser inmediato o diferido ${ }^{35}$.

Los ojos sin tratamiento previo tuvieron resultados mucho mejores que aquellos con tratamiento previo. Esto se atribuye a los cambios estructurales irreversibles que se generan en la mácula en un edema macular crónico, apoyando el concepto de que se debe considerar una terapia multifuncional en aquellos pacientes no respondedores al tratamiento de forma temprana para evitar este tipo de complicaciones ${ }^{36}$.

Otro estudio que aporta datos trascendentes en cuanto al uso de Ozurdex ${ }^{\circledR}$ es el CHAMPLAIN, en el que se estudiaron 55 pacientes refractarios al tratamiento con antecedente de vitrectomía vía pars plana. En estos pacientes se aplicó Ozurdex ${ }^{\circledR} 0.7 \mathrm{mg}$ y la ganancia visual desde la basal fue de 6 letras después de 8 semanas de la aplicación y de 3 letras después de 26 semanas ${ }^{13,37}$.

En relación con los efectos adversos reportados, el incremento de la presión intraocular ocurrió en el 16\% de los pacientes y ninguno requirió tratamiento quirúrgico. La progresión de la catarata se reportó en el 17\% de los pacientes fáquicos y solo un paciente necesitó cirugía ${ }^{13,37}$.
Respecto a los efectos adversos observados en el estudio MEAD con 1048 pacientes, solo el $27.7 \%$ presentaron elevación de la presión intraocular $\geq 10 \mathrm{mmHg}$ de la basal. Un tercio de estos pacientes tratados con Ozurdex ${ }^{\circledR}$ requirieron tratamiento tópico para el control de la presión intraocular durante el periodo de estudio, y solo un paciente requirió tratamiento quirúrgico $(0.19 \%)$. Se observó que el perfil de elevación de la presión intraocular con Ozurdex ${ }^{\circledR}$ es predecible, presentándose a las 2 semanas, con un pico a los 2 meses y una disminución gradual a los 6 meses. Se demostró que estos aumentos son transitorios y manejables con tratamiento tópico hipotensor ${ }^{34}$.

Al respecto de la catarata, como ya se ha mencionado, los pacientes que presentaron una disminución de la visión atribuible a la catarata recuperaron las líneas de visión tras la cirugía de catarata. Tanto en el grupo de pacientes pseudofáquicos como fáquicos se observó disminución de la AVMC, lo que se atribuyó a la presencia de catarata. Esta disminución fue completamente recuperable y similar a la de pacientes que iniciaron tratamiento, incluyendo la extracción. A este respecto es importante considerar que el daño estructural a la retina resulta en pérdida de visión irreversible, por lo cual deberíamos considerar no priorizar la posibilidad de desarrollo de catarata sobre un daño irreversible a la retina (tabla 1$)^{34}$.

\section{Estudios en la vida real}

Se han realizado numerosos estudios en la vida real considerando el hecho de que los ensayos clínicos limitan la inclusión de pacientes por sus criterios de 
selección, los cuales en ocasiones son muy difíciles de aplicar a la práctica diaria, donde se enfrentan retos tales como el tipo de población y la posibilidad de continuidad del tratamiento a largo plazo, mismos que afectan la adherencia, y la carga de tratamiento tanto para pacientes como para proveedores.

En los estudios realizados por Kiss, et al. ${ }^{38}$ (2014), Bonnin, et al. ${ }^{39}$ (2015) y Talks, et al. ${ }^{40}$ (2016) se evaluó la terapia antiangiogénica para el EMD en la vida real, y a 12 meses se encontró que a menor número de inyecciones, con un promedio de 2.6 al año, la ganancia en letras era de 3.7, en comparación con aquellos pacientes que recibieron un promedio de siete inyecciones anuales y presentaron una ganancia de 8.5 letras, lo cual demuestra que un régimen intensivo genera mejores resultados. Así, en el análisis retrospectivo realizado por Kiss, et al. ${ }^{38}$ se demuestra que los pacientes que son tratados con anti-VEGF reciben en promedio menos de cuatro inyecciones al año.

En los estudios en la vida real sobre el tratamiento con Ozurdex ${ }^{\circledR}$ para el EMD hay diferentes datos de acuerdo con el tiempo de estudio del resultado en los pacientes. En los estudios realizados a 6 meses se observa una ganancia de 7.5 letras con un promedio de una aplicación, y de hasta 9.7 letras con 1.75 aplicaciones ${ }^{41.42}$. Los estudios realizados a 12 meses presentan una ganancia de 7.5 letras con un promedio de 1,27 aplicaciones (Chhablani, et al. ${ }^{43}$ ) y de 8.7 letras con un promedio de 2.13 aplicaciones a 1 año (Matonti, et al. $\left.{ }^{44}\right)$. Aknin, et al. ${ }^{45}$ reportan una ganancia de 16.5 letras con un promedio de 1.5 aplicaciones a 18 meses, y por último, Malcles, et al. ${ }^{46}$ reportan una ganancia de 9.5 letras con 3.6 aplicaciones a 36 meses.

Boyer, et al. ${ }^{34}$ reportan una mejor agudeza visual de forma mantenida con menor número de inyecciones. Se comparó Ozurdex ${ }^{\circledR}$ frente a placebo a 3 años de tratamiento, y el 22.2\% ( $n=351)$ de los pacientes presentaron una $A V M C \geq 15$ letras, en comparación con el $12 \%$ del grupo placebo $(n=350)$, en los que el promedio de inyecciones a 36 meses fue de 4.1 aplicaciones. Es importante notar que no se reportó una disminución de la ganancia obtenida después de 2 años.

Fraser-Bell, et al. ${ }^{47}$ realizaron un estudio de la vida real en el que se compararon Ozurdex ${ }^{\circledR}$ y bevacizumab valorando la AVMC y el GMC a 24 meses en ambos grupos ( $n=46$ y $n=42$, respectivamente). Se encontró que Ozurdex ${ }^{\circledR}$ proporcionó una ganancia visual comparable a la de la terapia antiangiogénica con bevacizumab en un periodo de 24 meses de $\geq 10$ letras, con un $43 \%$ en el grupo de Ozurdex ${ }^{\circledR}$ y un $45 \%$ en el de bevacizumab, con un promedio de cinco inyecciones en el grupo de Ozurdex ${ }^{\circledast}$ y de 13.9 en el de bevacizumab. En este mismo estudio se reportó el GMC, en el cual se observa una disminución inicial de mayor importancia en el grupo de Ozurdex ${ }^{\circledR}$, pero al final de los 24 meses fue muy similar en ambos grupos, concluyendo que se obtiene un resultado tanto anatómico como funcional equiparable, pero con una diferencia en el número de inyecciones muy importante.

El análisis post hoc del Protocolo I en el que se demostró que el edema persistente compromete la AVMC final del paciente y su respuesta al tratamiento nos lleva a pensar que el tratamiento temprano con Ozurdex ${ }^{\circledR}$ mejora de manera significativa los resultados de los pacientes. A este respecto, Escobar-Barranco, et al. ${ }^{41}$ llevaron a cabo un estudio de eficacia en la vida real en el que se compararon un grupo de pacientes con EMD sin tratamiento previo $(n=36)$ y un grupo con EMD refractario al tratamiento $(n=40)$; en ambos grupos se consideraron la AVMC y el GCM. El promedio de mejoría de la AVMC fue una línea de ETDRS [Early Treatment Diabetic Retinopathy Study] mayor en los pacientes sin tratamiento previo en comparación con aquellos con edema refractario, lo cual lleva a la conclusión de que la intervención temprana con dexametasona (Ozurdex ${ }^{\circledR}$ ) o el switch temprano del tratamiento pueden llevar a una mejoría de la AVMC y una reducción del edema tanto en los pacientes sin tratamiento previo como en aquellos con edema macular refractario al tratamiento (figura 8) 20,41. $^{2}$

En un metaanálisis de 16 estudios de la vida real que comparó 3859 pacientes tratados con terapia antiangiogénica por EMD recalcitrante con seis o más tratamientos previos de anti-VEGF intravítreo se demostró un efecto favorable, con una ganancia de 20 letras $y$ un seguimiento promedio a 6 meses ( 3 a 36 meses), en los pacientes a quienes se inició tratamiento con implante de dexametasona (figura 9$)^{48}$.

Veritti, et al. ${ }^{49}$ realizaron un estudio para correlacionar la disminución del edema mediante la medición del GMC por tomografía de coherencia óptica y los cambios iniciales de la AVMC dentro de los primeros días (72 horas) posteriores a la aplicación de Ozurdex ${ }^{\circledR}$ y hasta 90 días. En este estudio se demostró que el GMC empieza a disminuir en las primeras 72 horas posteriores a la aplicación, con un aproximado de la mitad de la reducción total ocurriendo dentro de este periodo. El estado de las capas externas de la retina se correlacionó fuertemente con la ganancia visual, observándose una ganancia en letras de 10 en promedio en la cartilla ETDRS al día 14 comparado con la basal.

Según los resultados reportados en los diferentes estudios se puede concluir que los esteroides resultan 


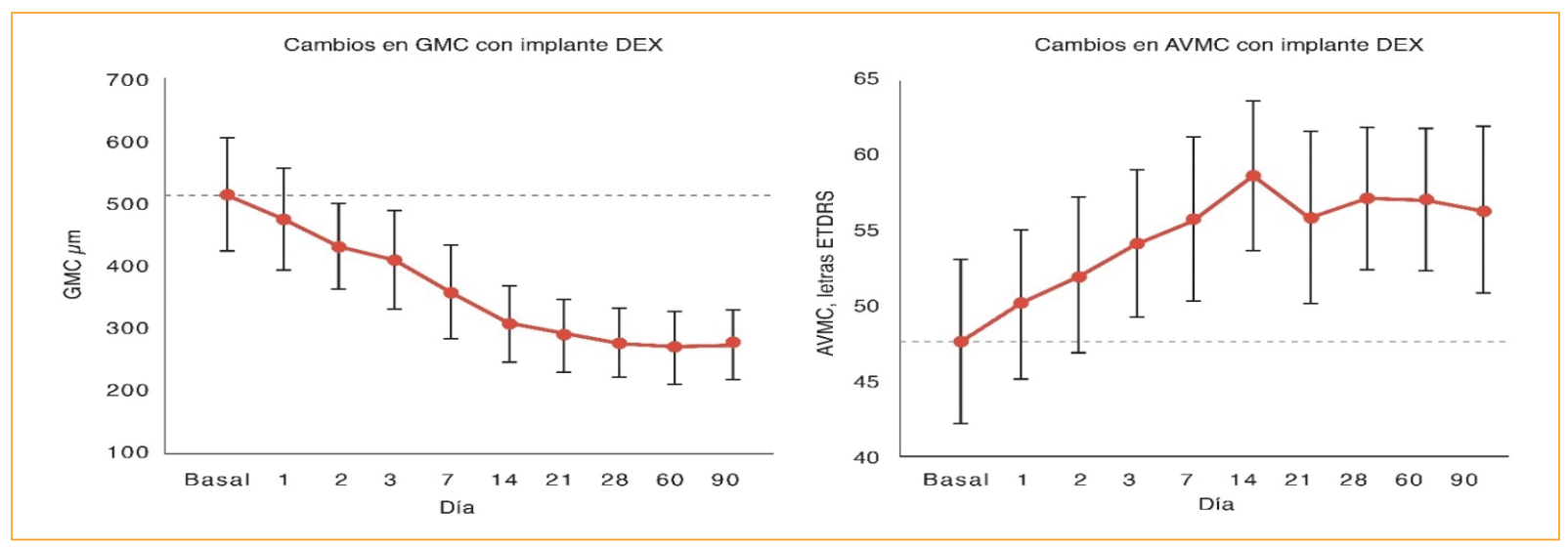

Figura 8. Correlación del grosor macular central (GMC) y la agudeza visual mejor corregida (AVMC) con los días posteriores a la aplicación del implante de dexametasona (DEX). ETDRS: Early Treatment Diabetic Retinopathy Study.

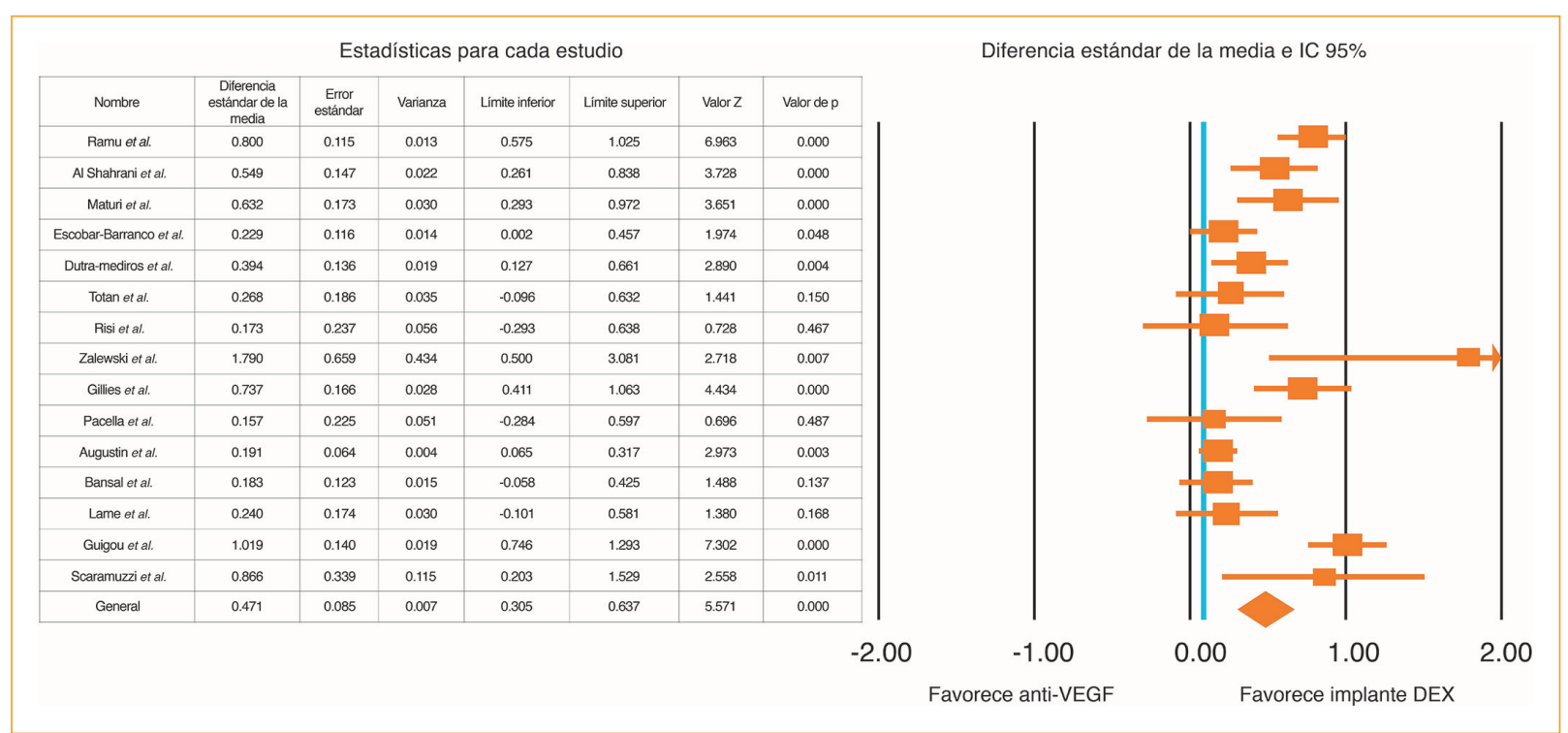

Figura 9. Diferencia estándar e intervalo de confianza del 95\% (IC 95\%) en metaanálisis de estudios de la vida real. Se observa una desviación a la derecha que indica una mejoría en los pacientes con edema macular diabético recalcitrante tratados con implante de dexametasona (DEX). VEGF: vascular endothelial growth factor.

ser una opción de tratamiento de gran importancia para el manejo del EMD, como primera línea de tratamiento en pacientes pseudofáquicos, en pacientes con afecciones cardiovasculares y en aquellos que no pueden mantener el apego al tratamiento (por aspectos económicos), y en segunda línea de tratamiento para los pacientes no respondedores al tratamiento después de tres inyecciones de anti-VEGF. Es indispensable tener en mente que en los pacientes que no responden a la terapia antiangiogénica después de tres a seis inyecciones se debe realizar un switch a esteroides lo antes posible para evitar una pérdida de visión irreversible por daño neuronal crónico ${ }^{34}$.
Los aspectos importantes que se deben considerar en el tratamiento de los pacientes con EMD son la agudeza visual inicial, la duración o la cronicidad del EMD para poder evaluar la posible respuesta y el pronóstico del paciente, los factores oculares patológicos locales relacionados (como podrían ser catarata, membranas epirretinianas o tracción), los factores sistémicos relacionados y la capacidad de respuesta variable al tratamiento.

Otro factor importante que se demostró en varios estudios de la vida real es que los efectos adversos sistémicos de estos agentes son nulos, ya que su circulación sistémica es muy limitada, y los efectos oculares relacionados, como aumento de la presión intraocular y catarata, son 
Tabla 1. Seguridad ocular en el EMD: implante DEX e implante FA. Seguridad ocular en los estudios MEAD y FAME a los 36 meses

\section{Implante DEX (0.7mg) MEAD}

\begin{tabular}{|c|c|c|}
\hline & $\begin{array}{l}\text { Implante DEX } \\
(0.7 \mathrm{mg}) \mathrm{MEAD}\end{array}$ & $\begin{array}{l}\text { Implante FA } \\
(0.2 \mu \mathrm{g}) \text { FAME }\end{array}$ \\
\hline Número de pacientes en el grupo del estudio & 351 & 375 \\
\hline Porcentaje de pacientes con PIO elevada como EA & $36 \%$ & $37.1 \%$ \\
\hline $\begin{array}{l}\text { Porcentaje de pacientes que requieren cirugía de } \\
\text { glaucoma / incisión }\end{array}$ & $0.6 \%$ & $4.8 \%$ \\
\hline $\begin{array}{l}\text { Porcentaje de pacientes con catarata antes de iniciar } \\
\text { el estudio }\end{array}$ & $85 \%$ & $44.3 \%$ \\
\hline $\begin{array}{l}\text { Porcentaje de pacientes que requieren cirugía de } \\
\text { catarata }\end{array}$ & $59 \%$ & $80 \%$ \\
\hline
\end{tabular}

completamente manejables y no resultan en una prioridad al compararse con los efectos que genera un EMD crónico sin el tratamiento oportuno ${ }^{34,41,50}$.

Actualmente, el esteroide de elección para el EMD es el implante de dexametasona de $0.7 \mathrm{mg}\left(\mathrm{Ozurdex}^{\circledR}\right)$, y en pacientes no respondedores a la dexametasona está descrito y autorizado en otros países el uso de acetato de fluocinolona (lluvien ${ }^{\circledR}$ ) por brindar un buen resultado en el EMD refractario al tratamiento y en cronicidad $>3$ años (este último no se encuentra disponible en México).

El implante de dexametasona (Ozurdex ${ }^{\circledR}$ ) es eficaz para tratar pacientes con EMD de corta y larga evolución, con una acción rápida y de larga duración, con un esquema de inyección bajo que resulta más práctico de manejar tanto para el paciente como para el médico. Constituye una alternativa viable para pacientes sin tratamiento previo y para aquellos previamente tratados, ya sea con láser o con terapia antiangiogénica, con ganancias en letras equiparable a la obtenida con la terapia antiangiogénica a 3 años y con menor número de tratamientos ${ }^{41,50,51}$.

\section{Bibliografía}

1. Bressler S, Ayala A, Bressler N, Melia M, Qin H, Ferris F, et al. Persistent macular thickening after ranibizumab treatment for diabetic macular edema with vision impairment. JAMA Ophthalmol. 2016;134:278-85.

2. Bressler N, Beaulieu W, Glassman A, Blinder K, Bressler S, Jampol L, et al. Persistent macular thickening following intravitreous aflibercept, bevacizumab or ranibizumab for central-involved diabetic macular edema with vision impairment: a secondary analysis of a randomized clinical trial. JAMA Ophthalmol. 2018;136:257-69.

3. Page C, Curtis M, Sutter M, Walker M, Hoffman B. Integrated pharmacology. London: Mosby; 1997. p. 83-90.

4. Buttgereit F, Da Silva J, Boers M, Burmester G, Cutolo M, Jacobs J, et al Standardized nomenclature for glucocorticoid dosages and glucocorticoid treatment regimens: current questions and tentative answers in rheumatology. Ann Rheum Dis. 2002;61:718-22.
5. Bringmann A, Pannicke T, Grosche J, Francke M, Wiedermann P, Skatchkov S, et al. Müller cells in the healthy and diseased retina. Prog Ret Eye Res. 2006;25:397-424

6. Uckermann O, Kutzera F, Wolf A, Pannicke T, Reichenbach A, Wiedemann $\mathrm{P}$, et al. The glucocorticoid triamcinolone acetonide inhibits osmotic swelling of retinal glial cells via stimulation of endogenous adenosine signaling. Pharmacol Exp Ther. 2005;315:1036-45.

7. Reichenbach A, Wurm A, Pannicke T, landiev I, Wiedemann P, Bringmann A. Muller cells as players in retinal degeneration and edema. Graef Arch Clin Exp Ophthalmol. 2007;245:627-36.

8. Kuppermann B, Zacharias N, Kinney M. Steroid differentiation: the safety profile of various steroids on retinal cells in vitro and their implications for clinical use (an American Ophthalmological Society Thesis). Trans Am Ophthalmol Soc. 2014;112:116-41.

9. Strehl C, Buttgereit F. Optimized glucocorticoid therapy: teaching old drugs new tricks. Molec Cel Endocrinol. 2013;380:32-40.

10. McCannel C. Meta-analysis of endophthalmitis after intravitreal injection of anti-vascular endothelial growth factor agents: causative organisms and possible prevention strategies. Retina. 2011;31:654-61.

11. Chawan-Saad J, Wu M, Wu A. Corticosteroids for diabetic macular edema. Taiwan J Ophthalmol. 2019;9:233-42.

12. Stewart M. Corticosteroid use for diabetic macular edema: ¿old fad or new trend? Curr Diab Rep. 2012;12:364-75.

13. Schmidt E, Garcia J, Bandello F, Berg K, Chakravarthy U, Gerendas B, et al. Guidelines for the management of diabetic macular edema by EURETINA. Ophthalmologica. 2017;237:185-222.

14. Edelman J. Differentiating intraocular corticoids. Ophthalmologica. 2010;224:25-30.

15. Kuppermann B, Blumenkranz M, Haller J, Williams G, Weinberg G, Chou C, et al. Randomized controlled study of an intravitreous dexamethasone drug delivery system in patients with persistent macular edema. Arch Ophthalmol. 2007:125:309-17.

16. Jonas J, Söfker A. Intraocular injection of crystalline cortisone as adjunctive treatment of diabetic macular edema. Am J Ophthalmol. 2001:132:425-7.

17. Thakur A, Kadam R, Kompella U. Trabecular meshwork and lens partitioning of corticosteroids: implications for elevated intraocular pressure and cataracts. Arch Ophthalmol. 2011:129:914-20.

18. Spandau U, Derse M, Schmitz P, Papoulis C, Jonas J. Dosage dependency of intravitreal triamcinolone acetonide as treatment for diabetic macular edema. Br J Ophthalmol 2005;89:999-1003.

19. Elman M, Aiello L, Beck R, Bressler N, Bressler S, Edwards A, et al. Randomized trial evaluating ranibizumab plus prompt or deferred laser or triamcinolone plus prompt laser for diabetic macular edema. Ophthalmology. 2010;117:1064-77.

20. Loewenstein $A$. The association between duration of edema and average vision improvement over three years: a post-hoc "area under curve" analysis of Protocol I. Retina World Congress, oral presentation.

21. Jonas J. Intravitreal triamcinolone acetonide for treatment of intraocular oedematous and neovascular diseases. Acta Ophthalmol Scand. 2005:83:645-63 
22. Ozkiris A, Erkilic K. Complications of intravitreal injections of triamcinolone acetonide. Can J Ophthalmol. 2005;40:63-8.

23. Wykoff C, Shah C, Dhoot D, Rodriguez H, Thompson D, Du W, et al Longitudinal retinal perfusion status in eyes with diabetic macular edema receiving intravitreal aflibercept or laser in VISTA study. Ophthalmol. 2019;126:1171-80.

24. Cantrill $\mathrm{H}$, Waltman $\mathrm{S}$, Palmberg $\mathrm{P}$, Zink H, Becker B. In vitro determination of relative corticosteroid potency. J Clin Endocrinol Metab. 1975;40:1073-7.

25. Campochiaro P, Hafiz G, Mahmood S, Bloom S, Brown D, Busquets M et al. Sustained ocular delivery of fluocinolone acetonide by an intravitreal insert. Ophthalmology. 2010;117:1393-9.

26. Jaffe G, Martin D, Callanan D, Pearson A, Levy B, Comstock T. Fluocinolone acetonide implant (Retisert) for noninfectious posterior uveitis: thirty-four-week results of a multicenter randomized clinical study. Ophthalmology. 2006;113:1020-7.

27. Cunha J, Ashton P, lezzi R, Campochiaro P, Digel P, Holz F, et al. Sustained delivery fluocinolone acetonide vitreous implants: long-term benefit in patients with chronic diabetic macular edema. Ophthalmology. 2014;121:1892-903.

28. Eaton A, Koh S, Jimenez J, Rieman C. The USER study: a chart review of patients receiving a $0.2 \mu \mathrm{g} /$ day fluocinolone acetonide implant for diabetic macular edema. Ophthalmol Ther. 2019;8:51-62.

29. Figueira J, Henriques J, Amaro M, Rosas V, Alves D, Cunha J. A non-randomized, open-label, phase 4 pilot study on the effect and safety of ILUVIEN ${ }^{\circledR}$ in chronic DME patients considered insufficiently responsive to available therapies (RESPOND). Ophthalmic Res. 2017:57:166-72.

30. Fusi W, Mukherjee C, Lane M, Tsaloumas M, Glover N, Kidess A, et al. Treating diabetic macular oedema (DMO): real world UK clinical outcomes for the $0.19 \mathrm{mg}$ fluocinolone acetonide intravitreal implant (lluvienTM) at 2 years. BMC Ophthalmol. 2018;18:62

31. Pochopien M, Beiderbeck A, McEwan P, Zur R, Toumi M, Aballea S. Cost-effectiveness of fluocinolone acetonide implant (ILUVIEN ${ }^{\circledR}$ ) in UK patients with chronic diabetic macular oedema considered insufficiently responsive to available therapies. BMC Health Serv Res. 2019;19:22.

32. Pearson P, Comstock T, Ip M, Callanan D, Morse L, Ashton P, et al. Fluocinolone acetonide intravitreal implant for diabetic macular edema: a 3-year multicenter, randomized, controlled clinical trial. Ophthalmology. 2011;118:1580-7.

33. Callanan D, Gupta S, Boyer D, Ciulla T, Singer M, Kuppermann B, et al Dexamethasone intravitreal implant in combination with laser photocoagulation for the treatment of diffuse diabetic macular edema. Ophthalmology. 2013;120:1843-51.

34. Boyer D, Yoon Y, Belfort R, Bandello F, Maturi R, Agustin A, et al. Threeyear, randomized, sham-controlled trial of dexamethasone intravitrea implant in patients with diabetic macular edema. Ophthalmology. 2014;121:1904-14.

35. Gonzalez V, Campbell J, Holecamp N, Kiss S, Lowenstein A, Augustin A, et al. Early and long-term responses to anti-vascular endothelial growth factor therapy in diabetic macular edema: analysis of Protocol I data. Am J Ophthalmol. 2016;172:72-9.
36. Castro V, Cervera E, Navarro C, Monferrer C, Hernández L, Montero J et al. Intravitreal dexamethasone implant Ozurdex ${ }^{\circledR}$ in naïve and refractory patients with different subtypes of diabetic macular edema. BMC Ophthalmol. 2019:19:15.

37. Boyer D, Faber D, Gupta S, Patel S, Tabandeh H, Li X, et al. Dexamethasone intravitreal implant for treatment of diabetic macular edema in vitrectomized patients. Retina. 2011;31:915-23.

38. Kiss S, Liu Y, Brown J, Holekamp N, Almony A, Campbell J, et al. Clinical utilization of anti-vascular endothelial growth-factor agents and patient monitoring in retinal vein occlusion and diabetic macular edema. Clin Ophthalmol. 2014;8:1611-21.

39. Bonnin S, Dupas B, Sanharawi M, Perol J, Erginay A, Tadayoni R, et al. Efficacy of dexamethasone intravitreal implant for the treatment of diabetic macular edema. Eur J Ophthalmol. 2015;25:448-53.

40. Talks J, Lotery A, Ghanchi F, Sivaprasad S, Johnston R, Patel N, et al. First-year visual acuity outcomes of providing aflibercept according to the VIEW study protocol for age-related macular degeneration.Ophthalmology 2016;123:337-43.

41. Escobar J, Pina B, Fernández M. Dexamethasone implants in patients with naïve or refractory diffuse diabetic macular edema. Ophthalmologica. 2015;233:176-85.

42. Medeiros M, Postorino M, Navarro R, García J, Mateo C, Corcostegui B. Dexamethasone intravitreal implant for treatment of patients with persistent diabetic macular edema. Ophthalmologica. 2014;231:141-6.

43. Chhablani J, Bansal P, Veritti D, Sambhana S, Sarao V, Pichi F, et al. Dexamethasone implant in diabetic macular edema in real-life situations. Eye. 2016;30:426-30.

44. Matonti F, Pommer S, Meyer F, Hajiar C, Merite P, Parrat E, et al. Longterm efficacy and safety of intravitreal dexamethasone implant for the treatment of diabetic macular edema. Eur J Ophthalmol. 2016;26:454-9.

45. Aknin I, Melki L. Longitudinal study of sustained-release dexamethasone intravitreal implant in patients with diabetic macular edema. Ophthalmologica. 2016;235:187-8

46. Malcles A, Dot C, Vorrin N, Agard E, Vie A, Bellocq D, et al. Real-life study in diabetic macular edema treated with dexamethasone implant: the Reldex study. Retina. 2017;37:753-60.

47. Fraser-Bell S, Lim L, Campain A, Mehta H, Aroni C, Bryant J, et al. Bevacizumab or dexamethasone implants for DME: 2-year results (the BEVORDEX study). Ophthalmol. 2016;123:1399-401.

48. Khan Z, Kuriakase R, Khan M, Chin E, Almeida D. Efficacy of the intravitreal sustained-release dexamethasone implant for diabetic macular edema refractory to anti-vascular endothelial growth factor therapy: meta-analysis and clinical implications. Ophthalmic Surg Lasers Imaging Retina. 2017;48:160-6.

49. Veritti D, Sarao V, Galiazzo F, Lanzetta P. Early effects of dexamethasone implant on macular morphology and visual function in patients with diabetic macular edema. Ophthalmologica. 2017;238:100-5.

50. Udaondo P. Ranibizumab in DME. AAO, New Orleans, USA; 2013. Poster PO213.

51. Guigou S, Hajjar C, Parrat E, Merite P, Pommer S, Matonti F, et al. Multicenter Ozurdex ${ }^{\circledR}$ assessment for diabetic macular edema: MOZART study. J Fr Ophthalmol. 2014;37:480-5. 


\title{
Catarata secundaria a esteroides
}

\author{
Ángeles Hernández-Vázquez* \\ Hospital de Nuestra Señora de la Luz, Ciudad de México, México
}

Los glucocorticoides son agentes antiinflamatorios muy efectivos utilizados ampliamente en el manejo de varias condiciones clínicas, incluyendo algunos trastornos oculares ${ }^{1}$.

En todo el mundo, la catarata es una de las principales causas de disminución visual (33\%) y la principal causa de ceguera (55\%). En la actualidad se considera que la catarata por esteroides es la cuarta causa más común de catarata secundaria. La edad es el mayor factor de riesgo $(73 \%)$ asociado a catarata; otros son la diabetes, la miopía y el uso crónico de esteroides. La posibilidad de presentar catarata secundaria al uso de esteroides aumenta conforme lo hace la expectativa de vida, por asociarse a un mayor uso y beneficio de los esteroides para el manejo de patologías asociadas $^{1-3}$.

Hoy en día se reconoce que la aplicación prolongada de esteroides por cualquier vía (intraocular, tópica o sistémica) se relaciona con el desarrollo de catarata subcapsular posterior. Aparentemente, la vía nasal tiene un rápido metabolismo y con frecuencia no se asocia a la aparición de este tipo de catarata (figura 1) 1 .

\section{Etiología}

El desarrollo de catarata no se restringe únicamente al uso de esteroides sistémicos; existen estudios que han reportado que el uso de esteroides tópicos también puede favorecer el desarrollo de catarata. Esto es un aspecto importante porque los esteroides tópicos

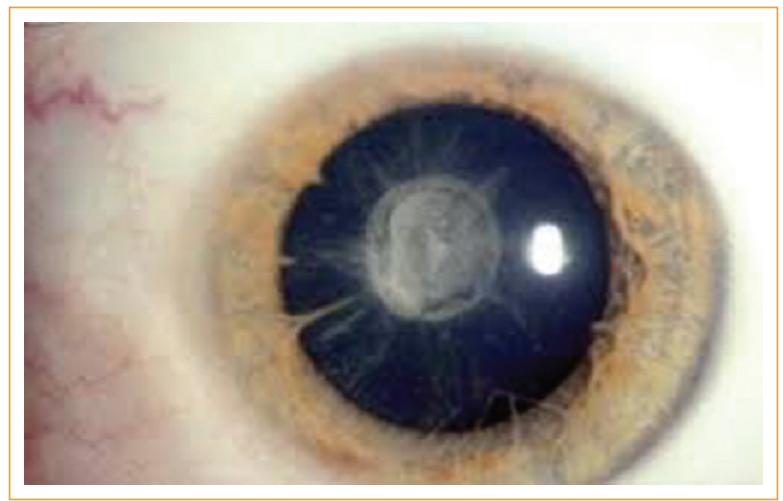

Figura 1. Catarata subcapsular posterior.

son ampliamente utilizados para el manejo de infecciones o enfermedades inflamatorias oculares. En la figura 2 se resumen algunos de los mecanismos del desarrollo de catarata por esteroides.

La catarata secundaria al uso de esteroides presenta unas características específicas:

- Solo se asocia a esteroides con actividad glucocorticoide, lo cual sugiere que el desarrollo de esta catarata es secundario a la activación de los receptores glucocorticoides. Los estudios han demostrado que el cristalino contiene receptores funcionales de glucocorticoides con la capacidad de inducir o reprimir la transcripción de genes que se sabe que están asociados con la activación del receptor de glucocorticoides en otro tipo de células. Varios estudios en cultivos de células epiteliales han demostrado que 


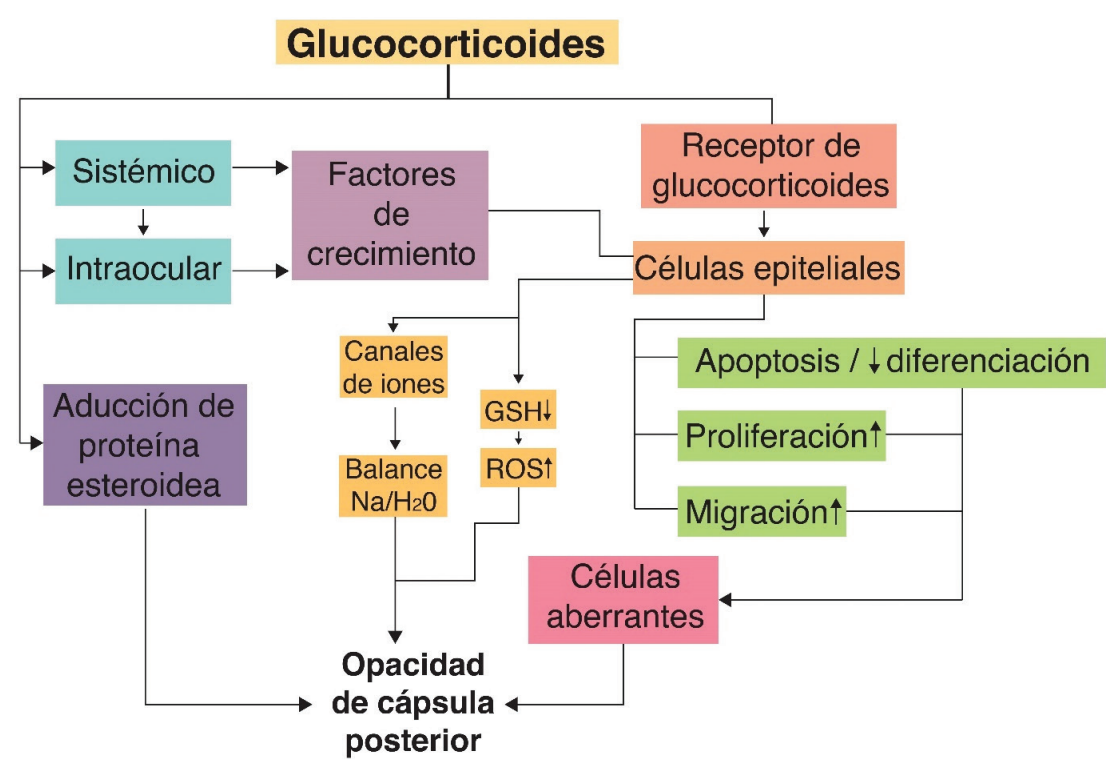

Figura 2. Formación de cataratas por el uso de esteroides.

después de 24 horas de la exposición a glucocorticoides existe una sobreexpresión de 57 transcriptasas y una subexpresión de aproximadamente 50 transcriptasas.

- Existe una migración posterior de las células epiteliales del cristalino del ecuador hacia la parte posterior. Las células epiteliales del cristalino se localizan en la cápsula anterior y el ecuador, pero en general están ausentes de la región subcapsular posterior entre el ecuador y el polo posterior del cristalino. El cristalino crece lentamente a lo largo de la vida, y se agregan nuevas fibras del cristalino a la corteza, por proliferación de las células epiteliales localizadas en una delgada banda en la zona germinal y transicional del ecuador del cristalino. Existen algunas teorías sobre el mecanismo de esta migración aberrante. Lyu, et al. ${ }^{4}$ sugirieron que la migración celular fuera de la región ecuatorial en cristalinos de ratas expuestas a glucocorticoides está relacionada con una reducción de E-cadherina, una proteína involucrada en la adhesión celular a la matriz extracelular y en la vía de señalización celular. Jobling y Augusteyn ${ }^{3}$ reportaron la presencia de una alteración en el gradiente normal de los factores de crecimiento, en particular del factor de crecimiento de fibroblastos-2, como un estímulo para la continua proliferación de células epiteliales del cristalino y su posterior migración dirigida.
- Su localización inicial está bien limitada a la parte central de la cápsula posterior del cristalino. También puede haber una sinergia entre la diabetes y el tratamiento con glucocorticoides, lo que lleva a un desarrollo más temprano y más agresivo de opacificación de la cápsula posterior (OCP). Las similitudes entre la OCP inducida por esteroides y la OCP relacionada con diabetes coinciden con la posibilidad de que pueda haber elementos comunes a los mecanismos subyacentes al inicio de la OCP diabética y la OCP por esteroides.

\section{Otras teorías}

\section{Oxidación e hidratación del cristalino}

La oxidación de las proteínas del cristalino y la disrupción de la hidratación del cristalino son mecanismos generales asociados a las cataratas relacionadas con la edad. Se ha sugerido que estos mecanismos posiblemente sean importantes en la inducción de cataratas con esteroides. La disminución de la concentración de glutatión en su forma reducida es una característica común de los cristalinos con catarata, y por lo tanto se esperaría que este fenómeno promoviera la oxidación de las proteínas del cristalino; sin embargo, se sabe poco sobre la influencia del tratamiento con esteroides en la oxidación de las proteínas del cristalino, pero se considera que esta debe tomarse en cuenta'. 
Es posible que la administración de glucocorticoides pueda conducir directamente a cambios en la hidratación del cristalino mediante la modificación de la expresión de los canales de la membrana de la superficie celular, los cuales son importantes en la regulación de los iones intracelulares y el agua. Por ejemplo, el tratamiento con glucocorticoides sistémicos afecta directamente el grado de expresión de la Na/K-ATPasa y el equilibrio del agua en otros tipos de células. Sin embargo, estos mecanismos aún no se han explorado en la catarata secundaria a esteroides ${ }^{1}$.

\section{Uniones proteína-esteroide}

La hipótesis de que la OCP inducida por esteroides se produce por la unión de los esteroides a las proteínas del cristalino para generar uniones de proteínas-esteroides ha recibido la mayor atención como posible causa de OCP. Originalmente atribuida a Bucala, et al. ${ }^{5}$, esta hipótesis sugiere que el grupo carbonilo C-20 del esteroide forma una base de Schiff con un grupo residuo amino del residuo lisina de cristalina del cristalino. En contraste, Jobling y Augusteyn ${ }^{3}$ notaron que existía un problema no resuelto: si la formación de estas uniones está involucrada en la formación de cataratas por esteroides, ¿por qué estas uniones estarían restringidas a los extremos terminales de las fibras del cristalino solo en el polo posterior? No obstante, como hay evidencia de cierta formación de uniones proteína-esteroide, este mecanismo podría contribuir al desarrollo de cataratas.

\section{Catarata y triamcinolona}

El acetónido de triamcinolona es un agonista glucocorticoide de administración intravítrea utilizado en enfermedades retinianas asociadas a proliferación celular anormal y alteraciones retinianas con acúmulo de líquido intrarretiniano o subretiniano, como es el caso del edema macular diabético (EMD). La mayoría de los estudios tienen un seguimiento de entre 3 y 12 meses, por lo que la incidencia de progresión de catarata puede variar. La mayoría de los estudios encuentran una incidencia de progresión de catarata de hasta el $25-28 \%$, y la cirugía de facoemulsificación se reporta en un $15-20 \%$ después de 1 año de uso de triamcinolona intravítrea. Algunos expertos opinan que la incidencia de efectos adversos depende de la dosis y de la presentación (con o sin conservadores). En un reporte se describió que la incidencia de progresión de catarata no fue mayor utilizando diferentes dosis de triamcinolona intravítrea, por lo que se concluyó que esta incidencia es independiente de la dosis y de la frecuencia de administración ${ }^{6-9}$.

\section{Catarata y fluocinolona}

El implante de acetónido de fluocinolona libera una dosis baja de fármaco hasta por 18036 meses. La dosis liberada es de $0.200 .5 \mu \mathrm{g}$ de fluocinolona por día. A los 24 meses, aproximadamente el $75 \%$ de los pacientes que eran fáquicos al inicio del estudio ${ }^{8,9}$ habían sido sometidos a cirugía de cataratas. La catarata se observó principalmente entre los meses 6 y 18.

\section{Catarata y dexametasona}

Ozurdex ${ }^{\circledR}$ es un implante de dexametasona de liberación lenta aprobado por la Food and Drug Administration para el tratamiento de uveítis, infecciones, edema macular por oclusión venosa y EMD. Uno de sus efectos secundarios reportados en los ensayos clínicos es el desarrollo de cataratas. La progresión de las cataratas es otra consideración importante con el uso de implantes de dexametasona. En el estudio GENEVA no se observó una progresión significativa en la formación de cataratas en el seguimiento a 6 meses $^{10}$.

El estudio MEAD reportó un $67.9 \%$, un $64.1 \%$ y un $20.4 \%$ de pacientes con catarata visualmente significativa posterior al implante de $0.7 \mathrm{mg} 00.35 \mathrm{mg}$ de dexametasona o placebo, respectivamente, al final de los 3 años del estudio. La tasa de cirugía de cataratas durante el estudio fue del $59.2 \%$, el $52.3 \%$ y el $7.2 \%$, respectivamente, pero la mayoría de las cataratas fueron visualmente significativas durante el primer año ${ }^{11}$.

En el estudio BEVORDEX se encontró un aumento de dos grados en la progresión de cataratas a los 12 meses en el $13 \%$ de los pacientes tratados con implantes de dexametasona y el $4.8 \%$ de los tratados con bevacizumab. Comparando el implante de dexametasona con la triamcinolona intravítrea, el $12.5 \%$ y el $16.6 \%$ de los pacientes, respectivamente, requirieron cirugía de cataratas durante 1.5 años de seguimiento ${ }^{12}$.

La tasa de progresión de cataratas y cirugía en diversos estudios se presenta en la tabla 1.

De acuerdo con esta información presentada, no es del todo certero atribuir en la mayoría de los estudios la formación de catarata al uso de un esteroide, ya que una gran proporción de estos pacientes tienen otros factores de riesgo, como uso de esteroides sistémicos, 
Tabla 1. Progresión de catarata con el implante de dexametasona

\begin{tabular}{|l|c|c|c|c|c|}
\hline Estudio & $\begin{array}{c}\text { Duración } \\
\text { (meses) }\end{array}$ & $\begin{array}{c}\text { Pacientes } \\
\text { fáquicos }\end{array}$ & $\begin{array}{c}\text { Dosis (mg) } \\
\text { \% progresión de } \\
\text { catarata }\end{array}$ & $\begin{array}{c}\text { \% cirugía durante } \\
\text { el estudio }\end{array}$ \\
\hline GENEVA: Haller, et al. 2010 & 6 & 735 & $0.7 / 0.35$ & 6.5 & 0.4 \\
\hline GENEVA: Haller, et al. 2011 & 12 & 302 & $0.7 / 0.35$ & $29.8 / 19.8$ & $1.3 / 1.8$ \\
\hline HURON: Lowder, et al. 2011 & 6.5 & 113 & $0.7 / 0.35$ & 13.2 & 2.6 \\
\hline PLACID: Callanan, et al. 2013 & 12 & 103 & 0.7 & 22.2 & 4.4 \\
\hline SHASTA: Capone, et al. 2014 & $3-6$ & 128 & 0.7 & NR & 35.9 \\
\hline MEAD: Boyer, et al. 2014 & 36 & 524 & $0.7 / 0.35$ & $67.9 / 64.1$ & $59.2 / 52.3$ \\
\hline BEVORDEX: Gillies, et al. & 12 & 46 & 0.7 & 35 & 4.8 \\
\hline ERIE: Kuppermann & 6 & 123 & 0.7 & 6.6 & NR \\
\hline OZDRY: Ramu, et al. 2015 & 12 & 73 & 0.7 PRN o fijo & 78.1 & 6.8 \\
\hline OMAR: Ozkok, et al. 2015 & 18 & 8 & 0.7 & NR & 12.5
\end{tabular}

NR: no reportado; PRN: por razón necesaria.

diabetes tipo 20 antecedente de vitrectomía. La progresión de catarata o la formación de catarata en un cristalino claro debido al uso de un esteroide intravítreo es uno de los efectos secundarios que pueden ocasionar baja visual, la cual va a depender del número de implantes colocados durante el primer año (más de dos implantes) y del estado del cristalino previo al tratamiento. Por lo tanto, los pacientes deben ser asesorados sobre la tasa de formación de cataratas e idealmente evaluados como candidatos para cirugía de cataratas antes de iniciar el tratamiento ${ }^{13}$.

\section{Bibliografía}

1. James E. The etiology of steroid cataract. J Ocular Pharmacol Ther. 2017; 23:403-20.

2. Pascolini D, Mariotti S. Global estimates of visual impairment: $2010 . \mathrm{Br}$ J Ophthalmol 2012;96:614-8.

3. Obling A, Augusteyn R. What causes steroid cataracts? A review of steroid induced posterior subcapsular cataracts. Clin Exp Optom. 2002;85:61-75.

4. Lyu J, Kim J, Chung S, Kim K, Joo C. Alteration of cadherin in dexamethasone-induced cataract organ-cultured rat lens. Invest Ophthalmol Vis Sci. 2003;44:2034-40
5. Bucala R, Gallati M, Manabe S, Cotlier E, Cerami A. Glucocorticoid-lens protein adducts in experimentally induced steroid cataracts. Exp Eye Res. 1985;40:853-63.

6. Veritti D, Di Giulio A, Sarao V, Lanzetta P. Drug safety evaluation of intravitreal triamcinolone acetonide. Expert Opin Drug Saf. 2012;11: 331-40.

7. Gillies M, Simpson J, Billson F, Luo W, Penfold P, Chua W, et al. Safety of an intravitreal injection of triamcinolone. Arch Ophthalmol. 2004;122:336-40.

8. Jonas J, Degenring R, Vossmerbauemer U, Kamppeter B. Frequency of cataract surgery after intravitreal injection of high-dosage triamcinolone acetonide. Eur J Ophthalmol. 2005;15:462-4.

9. Lam D, Chan C, Mohamed S, Lai T, Li K, Li P, et al. A prospective randomized trial of different doses of intravitreal triamcinolone for diabetic macular oedema. Br J Ophthalmol. 2007:91:199-203.

10. Haller J, Bandello F, Belfort R, Blumenkranz M, Gillies M, Heier J, et al. Randomized, sham-controlled trial of dexamethasone intravitreal implant in patients with macular edema due to retinal vein occlusion. Ophthalmology. 2010:117:1134-46.

11. Boyer D, Yoon Y, Belfort R, Bandello F, Maturi R, Agustin A, et al. Threeyear, randomized, sham controlled trial of dexamethasone intravitreal implant in patients with diabetic macular edema. Ophthalmology. 2014;121:1904-14

12. Gillies M, Lim L, Campain A, Quin G, Salem W, Li J, et al. A randomized clinical trial of intravitreal bevacizumab versus intravitreal dexamethasone for diabetic macular edema: the BEVORDEX study. Ophthalmology. 2014;121:2473-81.

13. Lam W, Albiani D, Yoganathan $P$, Chen J, Kherani A, Maberley D, et al. Real-world assessment of intravitreal dexamethasone implant $(0.7 \mathrm{mg})$ in patients with macular edema: the CHROME study. Clin Ophthalmol. 2015:9:1255-68. 


\title{
Edema macular diabético y retinopatía diabética proliferativa
}

\author{
Jesús González-Cortés* \\ Hospital Universitario José Eleuterio González, Universidad Autónoma de Nuevo León, Monterrey, Nuevo León, México
}

La retinopatía diabética $(\mathrm{RD})$ se caracteriza por el daño progresivo en los capilares retinianos y posteriormente por la isquemia retiniana, que en casos graves conduce a una RD que amenaza la visión inducida por angiogénesis y fuga de líquidos. El factor de crecimiento endotelial vascular (VEGF, vascular endothelial growth factor) es clave en el desarrollo y la progresión de la RD y del edema macular diabético (EMD), el cual es regulado en gran medida por la tensión de oxígeno $0^{1-3}$.

El Early Treatment Diabetic Retinopathy Study (ETDRS) demostró que la fotocoagulación focal del EMD clínicamente significativo reduce de manera sustancial el riesgo de pérdida de visión. El tratamiento focal también aumenta las posibilidades de mejoría visual, disminuye la frecuencia de EMD persistente y causa solo pérdidas menores del campo visual. Por otro lado, la fotocoagulación panretiniana (FPR) ha sido el tratamiento estándar para la RD proliferativa (RDP) desde que el ETDRS demostró su beneficio hace 45 años. Sin embargo, la FPR puede causar pérdida permanente del campo visual periférico y disminución de la visión nocturna, además de exacerbar el EMD, lo que lleva a la necesidad de tratamientos alternativos ${ }^{4-6}$.

Numerosos estudios sobre el tratamiento con inhibidores del VEGF (anti-VEGF) han demostrado consistentemente su eficacia en el tratamiento del EMD. Por ello, estos agentes se han posicionado como la terapia de primera línea para el EMD, con resultados de agudeza visual superiores y riesgos aceptables en comparación con el láser focal, en rejilla o sin tratamiento, y ha llevado a la observación de que las lesiones de la RD pueden revertirse con el tratamiento ${ }^{7-14}$.

\section{Protocolos en el tratamiento del edema macular diabético y la retinopatía diabética proliferativa}

En los resultados del Protocolo S de DRCR.net, el ranibizumab intravítreo cumplió con el resultado de no inferioridad en el cambio de la agudeza visual mejor corregida (AVMC) a los 2 años de seguimiento, que no fue peor que en el grupo de FPR para el tratamiento de la RDP. No hubo diferencias estadísticamente significativas en la AVMC entre los grupos de ranibizumab y de FPR (figura 1), con el reconocimiento de que el $53 \%$ del grupo de FPR recibió inyecciones adicionales de ranibizumab para tratar el EMD y solo el $6 \%$ del grupo del ranibizumab requirieron FPR. Se produjo una mayor pérdida de campo visual periférico (intervalo de confianza del 95\% [IC95\%] para una diferencia: 213$531 \mathrm{~dB}$ ) y se realizaron más vitrectomías vía pars plana (VPP) (IC95\% para una diferencia: 4-15\%) en el grupo de FPR que en el grupo del ranibizumab. Además, los ojos tratados con ranibizumab eran menos propensos a desarrollar EMD con involucro central (EMD-IC) que causaba una discapacidad visual de 20/32 o peor; resultados similares a 1 año usando aflibercept en el ensayo clínico aleatorizado CLARITY. En sí, en el Protocolo $S$ de DRCR.net, un mayor número de pacientes en el grupo de FPR desarrolló EMD (28 frente a 9). En los resultados a 5 años, el promedio de inyecciones en el grupo de FPR fue de 7.9 y en el grupo de 


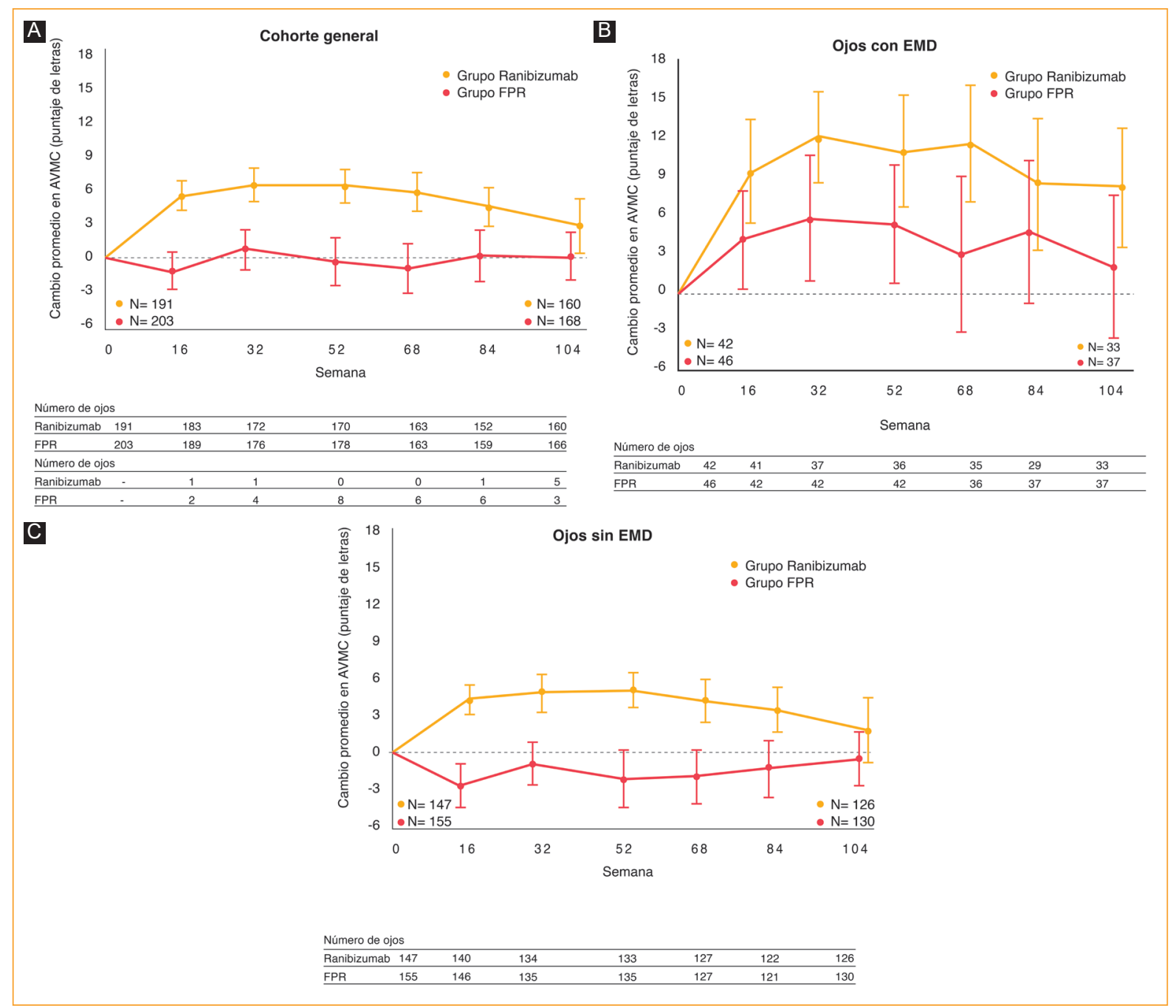

Figura 1. Cambio en la agudeza visual mejor corregida (AVMC) a lo largo del tiempo para la cohorte general, para ojos con y sin edema macular diabético (EMD) basal. FPR: fotocoagulación panretiniana.

ranibizumab fue de 19.2. La AVMC final media en ambos grupos fue de 20/25. A pesar de que a los 2 años el grupo de FPR tuvo mayor pérdida de campo visual, la disminución del campo visual periférico progresó en ambos grupos durante los posteriores 5 años de seguimiento ${ }^{15-17}$.

En un análisis post hoc del Protocolo T de DRCR. net, después de 2 años de seguimiento, se demostró una mejoría en la gravedad de la RD de aproximadamente el $25 \%$ con aflibercept, el $22 \%$ con bevacizumab y el $31 \%$ con ranibizumab en los pacientes con RD no proliferativa (RDNP) al inicio del estudio. A su vez, este análisis sugiere un beneficio secundario del tratamiento del EMD con aflibercept intravítreo con respecto a la mejoría de la gravedad de la RD en los pacientes con RDP desde el inicio del estudio (tabla 1) ${ }^{18}$.
Como previamente se mencionó, la terapia anti-VEGF para el EMD mejora la puntuación de la escala de gravedad de la $R D$ evaluada en fotografías de fondo a color y puede reducir su empeoramiento. Algunos ensayos aleatorizados que compararon inyecciones antiVEGF y FPR en la RDP demostraron la no inferioridad del anti-VEGF frente al FPR para prevenir complicaciones de la RDP, al menos durante los primeros 2 años. Sin embargo, la respuesta del anti-VEGF en la perfusión retiniana basada en angiografía con fluoresceína sigue sin estar clara. Estudios similares utilizando fotografías de campo ultraamplio y comparándolas con angiografía con fluoresceína de campo ultraamplio o angiografía por tomografía de coherencia óptica de barrido de campo amplio en ojos con RD y EMD concluyen que, después de las inyecciones con anti-VEGF, 
Tabla 1. Mejoría de la retinopatía diabética en las visitas anuales del grupo de tratamiento anti-VEGF y situación inicial de la retinopatía diabética

\begin{tabular}{|c|c|c|c|c|c|c|}
\hline \multirow[t]{2}{*}{ Características } & \multirow[t]{2}{*}{ Aflibercept } & \multirow[t]{2}{*}{ Bevacizumab } & \multirow[t]{2}{*}{ Ranibizumab } & \multicolumn{3}{|c|}{$\begin{array}{l}\text { Comparaciones entre grupos } \\
\text { pareados (ajustado IC } 95 \% \text { ) }\end{array}$} \\
\hline & & & & $\begin{array}{l}\text { Aflibercept } \\
\text { vs. } \\
\text { bevacizumab }\end{array}$ & $\begin{array}{l}\text { Aflibercept } \\
\text { vs. } \\
\text { ranibizumab }\end{array}$ & $\begin{array}{c}\text { Ranibizumab } \\
\text { vs. } \\
\text { bevacizumab }\end{array}$ \\
\hline $\begin{array}{l}\text { RDNP al inicio } \\
\text { No. ojos } \\
\text { Mejoría en } 1 \text { año } \\
\text { Ojos que mejoraron/ojos con } \\
\text { fotografías graduables } \\
\text { Mejoría \% } \\
\text { (IC 95\%) }\end{array}$ & $\begin{array}{c}167 \\
44 / 141 \\
31.2 \\
(23.7 \text { a } 39.5)\end{array}$ & $\begin{array}{c}147 \\
29 / 131 \\
22.1 \\
(15.4 \text { a } 30.2)\end{array}$ & $\begin{array}{c}163 \\
57 / 151 \\
37.7 \\
(30 \text { a } 46)\end{array}$ & $\begin{array}{c}11.7 \\
(2.9 \text { a } 20.6)\end{array}$ & $\begin{array}{c}2.9 \\
(-5.7 \text { a } 11.4)\end{array}$ & $\begin{array}{c}8.9 \\
(1.7 \text { a } 16.1)\end{array}$ \\
\hline Valor de $P$ ajustado & & & & 0.004 & 0.51 & 0.01 \\
\hline \multicolumn{7}{|l|}{ Mejoría en 2 años } \\
\hline $\begin{array}{l}\text { Ojos que mejoraron/ojos con } \\
\text { fotografías graduables }\end{array}$ & $33 / 133$ & $25 / 113$ & $40 / 129$ & & & \\
\hline Mejoría \% (IC 95\%) & $\begin{array}{c}24.8 \\
(17.7 \text { a } 33)\end{array}$ & $\begin{array}{c}22.1 \\
(14.9 \text { a } 30.9)\end{array}$ & $\begin{array}{c}31 \\
(23.2 \text { a } 39.7)\end{array}$ & $\begin{array}{c}3.1 \\
(-3.3 \text { a } 9.5)\end{array}$ & $\begin{array}{c}0.7 \\
(-6.4 \text { a } 7.7)\end{array}$ & $\begin{array}{c}2.4 \\
(-4 \text { a } 8.7)\end{array}$ \\
\hline Valor de $P$ ajustado & & & & 0.85 & 0.85 & 0.85 \\
\hline $\begin{array}{l}\text { RDP al inicio } \\
\text { No. ojos } \\
\text { Mejoría en } 1 \text { año } \\
\text { Ojos que mejoraron/ojos con } \\
\text { fotografías graduables } \\
\text { Mejoría \% (IC 95\%) }\end{array}$ & $\begin{array}{c}30 \\
22 / 29 \\
75.9 \\
(56.5 \text { a } 89.7)\end{array}$ & $\begin{array}{c}38 \\
11 / 35 \\
31.4 \\
(16.9 \text { a } 49.3)\end{array}$ & $\begin{array}{c}32 \\
16 / 29 \\
55.2 \\
(35.7 \text { a } 73.6)\end{array}$ & $\begin{array}{c}50.4 \\
(26.8 \text { a } 74)\end{array}$ & $\begin{array}{c}30 \\
(4.4 \text { a } 55.6)\end{array}$ & $\begin{array}{c}20.4 \\
(-3.1 \text { a } 44)\end{array}$ \\
\hline Valor de $P$ ajustado & & & & $<.001$ & 0.02 & 0.09 \\
\hline \multicolumn{7}{|l|}{ Mejoría en 2 años } \\
\hline $\begin{array}{l}\text { Ojos que mejoraron/ojos con } \\
\text { fotografías graduables }\end{array}$ & $19 / 27$ & $10 / 33$ & $9 / 24$ & & & \\
\hline Mejoría \% (IC 95\%) & $\begin{array}{c}70.4 \\
(49.8 \text { a } 86.2)\end{array}$ & $\begin{array}{c}30.3 \\
(15.6 \text { a } 48.7)\end{array}$ & $\begin{array}{c}37.5 \\
(18.8 \text { a } 59.4)\end{array}$ & $\begin{array}{c}35.9 \\
(6.1 \text { a } 65.6)\end{array}$ & $\begin{array}{c}31.4 \\
(-0.6 \text { a } 63.4)\end{array}$ & $\begin{array}{c}4.5 \\
(-20.5 \text { a } 29.4)\end{array}$ \\
\hline Valor de $P$ ajustado & & & & 0.01 & 0.06 & 0.73 \\
\hline
\end{tabular}

la mejoría en la puntuación de la escala de gravedad de la RD puede ocurrir sin reperfusión de vasos o capilaridad retiniana en la angiografía con fluoresceína de campo ultraamplio o en la angiografía por tomografía de coherencia óptica OCT de barrido de campo amplio. Por lo tanto, la fuerte correlación entre el número de lesiones en la RD y las zonas de no perfusión, establecida antes de cualquier tratamiento, podría no ser relevante después de la terapia anti-VEGF. Estos resultados deben tenerse en cuenta en futuros estudios, con el objetivo de demostrar una mejora en la perfusión retiniana periférica en la $R D$ después de la terapia anti-VEGF ${ }^{19-22}$.

\section{Algoritmo en el tratamiento de la retinopatía diabética proliferativa en diferentes escenarios}

Sun J, et al., basándose en los resultados del Protocolo $S$ de DRCR-net, sugieren un algoritmo para el tratamiento de la RDP en diversas circunstancias. Las distintas ventajas de cada modalidad de tratamiento pueden orientar la decisión clínica para cada caso en particular (tabla 2) ${ }^{15,23}$.

A continuación, se propone un algoritmo de tratamiento basado en la publicación de Sun J, et al. y en los resultados del Protocolo S de DRCR-net ${ }^{15,23}$. 
Tabla 2. Ventajas y desventajas relativas de los anti-VEGF en comparación con la FPR para el tratamiento de la retinopatía diabética proliferativa

\begin{tabular}{|l|l|}
\hline & Anti-VEGF \\
\hline $\begin{array}{l}\text { Número de } \\
\text { procedimientos para } \\
\text { tratamiento de RDP }\end{array}$ & $\begin{array}{l}\text { Se requieren múltiples inyecciones para respuesta } \\
\text { óptima (mediana de número de inyecciones en } 2 \text { años } \\
\text { fue } 14 \text { en ojos con EMD basal y } 10 \text { sin EMD basal) }\end{array}$ \\
\hline
\end{tabular}

Frecuencia de visitas subsecuentes

Agudeza visual a 2 años

Agudeza visual posterior a 2 años

Efecto en campo visual periférico

Efecto en estatus de EMD

Necesidad de un procedimiento adicional al tratamiento por RDP o EMD

Riesgo de eventos adversos asociados a procedimientos invasivos

Riesgo de hemorragia vítrea

Riesgo de desprendimiento traccional de retina

Riesgo de glaucoma

Riesgo de eventos adversos sistémicos

Costo beneficio

Consideraciones adicionales
Visitas mensuales o similar son necesarias en el primer año con un seguimiento subsecuente extendido si NV persiste

Agudeza visual sin disminución a 2 años de FPR (5 letras)

Agudeza visual promedio posterior a 2 años superior a FPR

Mejora estado de EMD y reduce el riesgo de aparición de EMD comparado con FPR

Mejora estatus de EMD y reduce el riesgo de aparición de EMD comparado con FPR

Pocos ojos que recibieron anti-VEGF según el algoritmo DRCR.net necesitarán FPR por empeoramiento o persistencia de NV. La necesidad de vitrectomía en los 2 años posteriores es menor en ojos tratados con anti-VEGF. Pocos ojos necesitarán tratamiento adicional de EMD

Procedimiento invasivo con riesgo asociado de endoftalmitis, desgarro de retina traumático 0 catarata

Posiblemente menor riesgo a 2 años

Posiblemente menor riesgo a 2 años. Posible aumento de riesgo de tracción de retina, pero menor riesgo de desprendimiento traccional de retina en ojos que no tienen tracción macular basa

Bajo riesgo de aumento sustancial de presión intraocular, necesidad de antihipertensivos oculares o cirugía de glaucoma

Riesgo teórico aumentado de eventos tromboembólicos y mayor riesgo de protocolo $\mathrm{S}$ con el grupo de ranibizumab han sido reportados, aunque el riesgo aumentado no ha sido demostrado de manera concluyente en estudios de anti-VEGF intravítreo por padecimientos oculares

Ranibizumab presente costo-beneficio adecuado comparado con FPR únicamente si existe EMD asociado

No se considera una buena opción en pacientes con comorbilidades sistémicas mayores en quienes la RDP puede recurrir o empeorar sustancialmente si no cumplen con un seguimiento mensual

\section{FPR}

Puede aplicarse en 1-2 sesiones, pero puede requerir tratamiento adicional $(45 \%$ de los ojos tratados con FPR necesitaron FPR adicional en los 2 años posteriores al tratamiento inicial)

Primera visita de seguimiento posterior a FPR normalmente programada a 3-4 meses con seguimiento subsecuente extendido si NV persiste $\cdots$

Agudeza visual promedio posterior a 2 años inferior a VEGF

Terapia destructiva que conlleva defectos en campo visual periférico

Riesgo mayor de EMD central de inicio comparado con anti-VEGF en 2 años. Aunado a EMD desarrollado posterior a FPR que puede llevar a baja visual

Pocos ojos que reciben FPR necesitarán anti-VEGF por NV persistente o empeoramiento. La necesidad de vitrectomía posterior a 2 años es mayor en ojos tratados con FPR. Algunos ojos pueden requerir anti-VEGF por empeoramiento o aparición de EMD

Procedimiento no invasivo sin riesgo de endoftalmitis y riesgo mínimo de desgarro de retina, desprendimiento de retina o catarata

Posiblemente mayor riesgo a 2 años

Posiblemente mayor riesgo de desprendimiento a 2 años. Aumento de tracción retiniana es posible pero menor riesgo de desprendimiento traccional de retina en ojos que no tienen tracción macular basal

Bajo riesgo de aumento sustancial de presión intraocular, necesidad de antihipertensivos oculares o cirugía de glaucoma. Efusión ciliocoroidea y glaucoma de ángulo cerrado son poco comunes, pero son eventos adversos posibles

Sin riesgo aumentado de eventos tromboembólicos u otros eventos sistémicos

PRP tiene mayor costo-beneficio comparado con ranibizumab en ojos con RDP sin EMD

Durabilidad del efecto prolongada para la regresión de NV que puede durar décadas. Excelente opción de tratamiento para RDP en pacientes con dificultad para mantener un seguimiento mensual 
Cuadro I. Algoritmo del protocolo S de DRCR-net para la retinopatía diabética proliferativa

1. Comenzar con seis inyecciones mensuales; si la neovascularización se resuelve después de cuatro o cinco inyecciones, las inyecciones pueden diferirse.

2. Después de 6 meses, continuar con las inyecciones si la neovascularización continúa progresando o sigue mejorando, pero diferir las inyecciones si la neovascularización se encuentra estable en la visita actual y en las últimas dos visitas ("estabilidad sostenida»).

3. Reanudar mensualmente el anti-VEGF si la neovascularización empeora después de suspender las inyecciones. Si se logra de nuevo la estabilidad sostenida, las inyecciones pueden diferirse una vez más, pero esto requiere al menos tres inyecciones consecutivas nuevamente: una administrada para el estado inicial de la neovascularización progresiva y dos más si la neovascularización se mantiene estable.

Cuadro II. Algoritmo para el tratamiento del edema macular diabético

1. Comenzar con tres inyecciones mensuales o hasta lograr la mejoría máxima.

2. Después de la fase de carga, continuar inyectando de acuerdo con una conducta reactiva o proactiva.

\section{Retinopatía diabética proliferativa sin edema macular diabético}

Según los resultados del Protocolo S de DRCR-net, tanto la FPR como la terapia anti-VEGF parecen ser opciones terapéuticas viables. Si bien la terapia antiVEGF es efectiva en la regresión de la neovascularización de la retina y en reducir el riesgo de desarrollar EMD, de manera global podría no ser rentable. En los Estados Unidos de América, el uso de agentes antiVEGF durante 2 años puede considerarse rentable dentro del rango de 50,000 a 150,000 dólares americanos por año de vida ajustado por calidad ${ }^{24}$.

- En caso de iniciar con anti-VEGF, se sugiere hacerlo de acuerdo con el algoritmo de tratamiento propuesto por el protocolo S (Cuadro I) y solo agregar la FPR si la neovascularización empeora de manera importante.

- En caso de iniciar con FPR, se sugiere agregar un anti-VEGF si la neovascularización empeora de manera importante (Cuadro I) o si aparece EMD (Cuadro II).

Deben considerarse las ventajas y desventajas de las opciones de tratamiento, además de las condiciones de cada paciente.

\section{Retinopatía diabética proliferativa con edema macular diabético sin involucro central}

En general, la terapia anti-VEGF ha sido aceptada como tratamiento de primera línea en el EMD, desplazando al láser como terapia de segunda línea. A pesar de que algunos autores sugieren la aplicación de láser en el EMD sin involucro central, existen reportes en los que la adición del láser convencional, subumbral o micropulsado no ha añadido beneficios a la monoterapia farmacológica en el EMD con y sin involucro centra| ${ }^{25-30}$

- En caso de iniciar con anti-VEGF para el EMD (Cuadro II), el uso de FPR puede diferirse ya que el mismo agente anti-VEGF tratará tanto el EMD como la RDP. Una vez que la terapia anti-VEGF no es necesaria, debido a la resolución o la estabilidad del EMD, estos ojos deben ser reevaluados en función del estado de la neovascularización. Si esta empeora de manera importante, se sugiere decidir la terapia como se ha indicado para la RDP sin EMD.

- En caso de nula o pobre respuesta del EMD pueden considerarse diversas opciones: cambio de anti-VEGF, láser macular, implante de dexametasona, etc. - En caso de iniciar con láser macular focal y FPR, se sugiere agregar un anti-VEFG si el EMD empeora (Cuadro II) o existe una progresión importante de la neovascularización (Cuadro I).

Deben considerarse las ventajas y desventajas de las opciones de tratamiento, además de las condiciones de cada paciente.

\section{Retinopatía diabética proliferativa con edema macular diabético con involucro central}

La terapia anti-VEGF se considera de primera línea en la mayoría de los ojos con EMD-IC. El ranibizumab fue altamente efectivo en el tratamiento de la RDP en el Protocolo $S$ de DRCR-net, y el aflibercept lo fue en el tratamiento de la RDP en el estudio CLARITY'15,16.

- El tratamiento del EMD-IC debe iniciarse con un anti-VEGF (Cuadro II). El uso de FPR puede diferirse, ya que el mismo agente anti-VEGF tratará tanto el EMD como la RDP. Una vez que la terapia anti-VEGF no es necesaria debido a la resolución o la estabilidad del EMD, estos ojos pueden ser reevaluados en función del estado de la neovascularización. Si esta empeora de manera importante, se sugiere decidir la terapia como se ha indicado para la RDP sin EMD. 
- En caso de nula o pobre respuesta del EMD pueden considerarse diversas opciones: cambio de anti-VEGF, láser macular, implante de dexametasona, etc.

Deben considerarse las ventajas y desventajas de las opciones de tratamiento, además de las condiciones de cada paciente.

\section{Retinopatía diabética proliferativa de alto riesgo con o sin edema macular diabético que perjudica la visión}

Para los ojos con RDP de alto riesgo, es decir, nivel de ETDRS $\geq 71$, que no requirieron VPP, las cifras de eventos de empeoramiento de la RDP (p. ej., hemorragia vítrea, desprendimiento de retina, neovascularización del ángulo o glaucoma neovascular) fueron mayores en comparación con los ojos que tenían una RDP de bajo riesgo independientemente del tratamiento. Sin embargo, el promedio de mejoría en la AVMC durante 2 años fue mayor con ranibizumab que con FPR, y la diferencia pareció ser mayor en los ojos con alto riesgo ${ }^{20,31}$.

- Debe considerarse al anti-VEGF como monoterapia para ojos con RDP (Cuadro I) incluso cuando se considere de alto riesgo y no se esté planificando una VPP inmediata.

\section{Empeoramiento de la retinopatía diabética proliferativa en ojos que reciben anti- VEGF (ranibizumab)}

Muy pocos ojos asignados al azar para recibir ranibizumab cumplieron con los criterios de falla para un empeoramiento sustancial de la RDP (3 de 191 ojos), y solo 12 ojos recibieron fotocoagulación panretiniana (FPR), 8 de los cuales durante la VPP. Estos resultados indican que los ojos tratados con ranibizumab para la RDP de acuerdo con el algoritmo del Protocolo $S$ de DRCR-net es poco probable que necesiten FPR para el tratamiento de la RDP. El tratamiento con anti-VEGF puede funcionar como monoterapia para la RDP, al menos durante los primeros 2 años si el cumplimiento por parte del paciente es bueno. Sin embargo, se observó un empeoramiento de la neovascularización en aproximadamente un $13 \%$ de las visitas, con un empeoramiento posterior en seguimiento de 2 años. Por lo tanto, mayores aplicaciones de FPR para la RDP que progresa podrían resultar en una dosificación menos frecuente 0 intervalos de seguimiento más largos que los utilizados en el Protocolo S de DRCR-net.

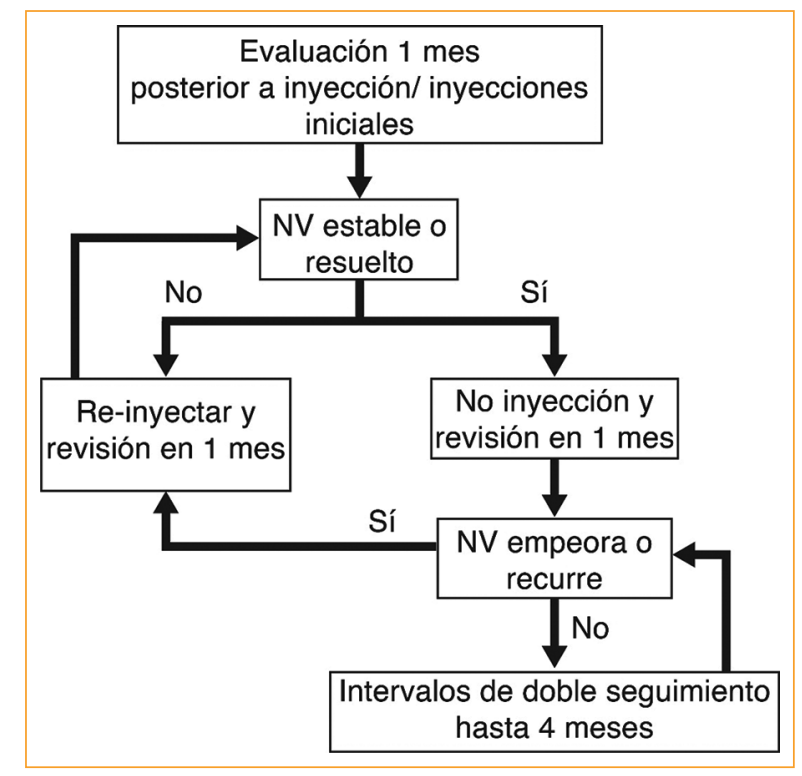

Figura 2. Principios de la DRCR.net. Algoritmo de tratamiento con anti-VEGF para la retinopatía diabética proliferativa.

\section{Vitrectomía para la retinopatía diabética proliferativa}

La VPP se permitió en el Protocolo S de DRCR-net para el desprendimiento de retina traccional con deterioro visual o hemorragia vítrea persistente. Sin embargo, se pidió a los investigadores que difirieran la cirugía durante al menos 8 semanas después del inicio de la hemorragia vítrea. En los ojos tratados con ranibizumab, en comparación con los tratados con FPR, hubo menos hemorragias vítreas $(27 \%$ frente a $34 \%$; $p$ $=0.09)$, desprendimientos de retina $(6 \%$ frente a $10 \%$; $p=0.08)$ y VPP ( $4 \%$ frente a $15 \% ; p<0.001)$. Ocho ojos en el grupo de ranibizumab necesitaron VPP por los motivos siguientes: hemorragia vítrea persistente (6), desprendimiento de retina (1) y hemorragia vítrea con desprendimiento de retina (1). Debemos considerar que los ojos con desprendimiento de retina traccional e involucro macular y con VPP anticipada fueron excluidos del Protocolo $S$ de DRCR-net, y también de los Protocolos I, J y N. En consecuencia, se pidió a los investigadores que no incluyeran ojos con un riesgo significativo de neovascularización después de la terapia con anti-VEGF. Sin embargo, los resultados de estos estudios no respaldan la hipótesis de que el antiVEGF administrado a un ojo con RDP, con o sin características de alto riesgo (pero sin amenaza de desprendimiento de retina traccional con involucro 
macular desde el inicio del estudio), provoca neovascularización con mayor frecuencia que en los ojos con FPR. El protocolo $S$ de DRCR-net no comparó ranibizumab frente a FPR en ojos para los que ya se había planeado la VPP20.

La FPR se administra solo si la neovascularización empeora de manera sustancial a pesar del anti-VEGF. El inicio o el empeoramiento de la hemorragia prerretiniana o vítrea no se clasifica necesariamente como un empeoramiento de la neovascularización, a menos que la hemorragia impida la evaluación de la neovascularización. En la figura 2 y la tabla 2 se ofrece explicación adicional.

En la práctica clínica, es posible que el tratamiento de FPR con una o varias sesiones sea suficiente para controlar la RPD y no se requieran procedimientos adicionales. Por otro lado, el costo de la terapia con láser es menor que el de la terapia anti-VEGF, y además no presenta riesgo de endoftalmitis ni de eventos adversos sistémicos. El DRCR-net, en un análisis sobre costo-beneficio de la monoterapia con ranibizumab o con FPR para la RDP, observó que era más adecuado iniciar con FPR en pacientes con RDP sin EMD asociado y con ranibizumab en aquellos con EMD al momento de la detección. Por lo tanto, los beneficios relativos del tratamiento de la RDP con anti-VEGF frente a FPR podrían considerarse en un paciente que presenta EMD, en el cual la terapia anti-VEGF generalmente es necesaria, siempre y cuando el paciente se apegue al tratamiento y tenga posibilidades para acceder a él ${ }^{15,32,33}$.

En México es posible adoptar el algoritmo del Protocolo S de DRCR-net; sin embargo, las circunstancias de nuestros pacientes y de nuestro entorno podrían modificar el esquema. Deben considerarse las ventajas y las desventajas de las opciones de tratamiento, además de las condiciones socioeconómicas, de apego al tratamiento y de acceso a medicamentos fuera de su indicación (off-label).

Es de conocimiento general que la patogénesis y la progresión de la RD implican cambios en la estructura vítrea y su relación con la interfase vitreorretiniana. Un estudio cuyo objetivo fue evaluar los costos y la utilidad de la VPP temprana en comparación con la FPR y el ranibizumab intravítreo en pacientes con RDP sin EMD, mediante un análisis de decisión basado en los resultados del Protocolo S de DRCR-net a 2 años de tratamiento para cada escenario, concluyó que la VPP como estrategia de tratamiento demuestra una utilidad de costo similar al manejo con FPR y una utilidad de costo más favorable en comparación con la terapia con ranibizumab intravítreo a corto plazo. Esta ventaja sobre el

\section{Cuadro III. Protocolos en curso}

- Protocolo V: estudio aleatorizado de ojos con EMD y AVMC $\geq$ 20/25. Tratamiento con aflibercept y ziv-aflibercept inmediato o láser pronto con anti-VEGF diferido, para evaluar los ojos con pérdida de $\geq 5$ letras en 2 años.

- Protocolo W: seguridad y eficacia del aflibercept en comparación con observación en RDNP grave y AVMC $\geq$ 20/25 sin EMD y sin tratamiento previo, para valorar la aparición de edema o el avance de la retinopatía.

- Protocolo AA: evaluación de lesiones periféricas en la RD y su asociación con el avance de la retinopatía en pacientes con RDNP, sin EMD ni tratamiento previo, comparando imágenes de campo ultraamplio frente a fotografías estándar de los siete campos (ETDRS) con el objetivo de determinar si las fotografías de campo ultraamplio aportan mayor información que las convencionales.

- Protocolo AB: se compara el tratamiento con VPP temprana frente a aflibercept en el hemovítreo secundario a RDP mediante la evaluación de la AVMC a 6 meses de tratamiento.

- Protocolo AC: evaluación de los cambios en la AVMC en pacientes con terapia anti-VEGF (aflibercept frente a bevacizumab + aflibercept diferido) para el EMD-IC.

- Protocolo AD (PROMINENT): evaluación de si el tratamiento con pemfibrato ( $0.2 \mathrm{mg} / 12 \mathrm{~h}$ vía oral), comparado con placebo, reduce la tasa de empeoramiento de la $\mathrm{RD}$ en pacientes con diabetes tipo 2 y RDNP.

- Protocolo GEN: creación de un banco de material genético y de información sobre el fenotipo clínico que permita evaluar la susceptibilidad o resistencia genética en la RD, y determinar variantes sobre biomarcadores clave en el desarrollo de EMD y neovascularización.

anti-VEGF es continua cuando se tienen en cuenta los costos de por vida. La seguridad de los anti-VEGF en comparación con la VPP primaria (sin anti-VEGF) para la hemorragia vítrea persistente se está evaluando en el Protocolo $A B^{34-41}$.

El estudio PANORAMA, de fase 3 , aleatorizado y doble ciego, cuyo objetivo es evaluar la eficacia y la seguridad de la inyección intravítrea de aflibercept en comparación con un tratamiento simulado en la mejoría de la RDNP moderada a grave en ausencia de EMD-IC, demuestra en la semana 24 que el aflibercept mejora la gravedad de la RD en los pacientes con RDNP moderadamente grave o grave, y sugiere que el antiVEGF puede revertir la progresión de la enfermedad en estos pacientes ${ }^{42}$.

En el caso del EMD, la pobre o nula respuesta al anti-VEGF utilizado y la relación de este con la isquemia periférica retiniana persistente requieren modificaciones en el tratamiento. Deben considerarse alternativas como cambiar (switch) de anti-VEGF, utilizar un implante de dexametasona intravítreo, aplicar FPR adicional de la retina periférica, realizar una VPP o combinar tratamientos. Estos aspectos se tratan en otros capítulos de este consenso. 
Por otro lado, aunque la monoterapia con anti-VEGF logre la estabilización de la neovascularización en la RDP, agregar FPR (combinar) podría resultar en una menor frecuencia de aplicaciones intravítreas, lo que resulta en menor riesgo y menos costo para el paciente.

En general, podemos concluir que la meta para lograr el éxito en el tratamiento de la RDP y del EMD es la inhibición del VEGF y de los factores proinflamatorios, condición que parece obtenerse de manera más eficaz con la terapia farmacológica en comparación con la ablación retiniana. En la actualidad, las indicaciones del láser, de la terapia farmacológica intravítrea (anti-VEGF y antiinflamatorios esteroideos) y de la VPP cada vez son más claras. Así, los anti-VEGF parecen perfilarse como terapia de primera línea en la RDP, como también se sugiere en el tratamiento del EMD. Es posible que los esquemas de tratamiento en los pacientes con RDNP grave con o sin EMD sean indiferentes a los sugeridos actualmente en los pacientes con RDP con o sin EMD; inclusive que la VPP primaria temprana sea una alternativa para prevenir las complicaciones retinianas de la patología microvascular diabética. Actualmente existen diversos protocolos en curso (Cuadro III) cuya finalidad es entender de manera más exacta el comportamiento de la RDP y del EMD ante diversos escenarios y aportar bases más sólidas para un esquema de tratamiento eficaz y oportuno.

\section{Bibliografía}

1. Kohner E. Diabetic retinopathy. BMJ. 1993;307:1195-9.

2. Ferrara N. Molecular and biological properties of vascular endothelial growth factor. J Mol Med. 1999;77:527-43.

3. Stitt A, Curtis T, Chen M, Medina R, McKay G, Jenkins A, et al. The progress in understanding and treatment of diabetic retinopathy. Prog Retin Eye Res. 2016;51:156-86.

4. Photocoagulation for diabetic macular edema. Early Treatment Diabetic Retinopathy Study report number 1. Early treatment diabetic retinopathy study research group. Arch Ophthalmol. 1985;103:1796-806

5. Diabetic Retinopathy Study Research Group. Photocoagulation treatment of proliferative diabetic retinopathy: clinical application of Diabetic Retinopathy Study (DRS) findings, DRS report number 8. Ophthalmology. 1981;88:583-600.

6. Brucker A, Qin H, Antoszyk A, Beck R, Bressler N, Browning D, et al. Observational study of the development of diabetic macular edema following panretinal (scatter) photocoagulation given in 1 or 4 sittings. Arch Ophthalmol. 2009;127:132-40.

7. Googe J, Brucker A, Bressler N, Qim J, Aiello L, Antonszyk A, et al. Randomized trial evaluating short-term effects of intravitreal ranibizumab or triamcinolone acetonide on macular edema after focal/grid laser for diabetic macular edema in eyes also receiving panretinal photocoagulation. Retina. 2011;31:1009-27.

8. Do D, Nguyen Q, Boyer D, Schmidt U, Brown D, Vitti R, et al. One-year outcomes of the da Vinci Study of VEGF Trap-Eye in eyes with diabetic macular edema. Ophthalmology. 2012;119:1658-65.

9. Heier J, Korobelnik J, Brown D, Schmidt U, Do D, Midena E, et al. Intravitreal aflibercept for diabetic macular edema: 148-week results from the VISTA and VIVID Studies. Ophthalmology. 2016;123:2376-85.

10. Wells J, Glassman A, Ayala A, Jampol L, Bressler N, Bressler S, et al. Aflibercept, bevacizumab or ranibizumab for diabetic macular edema: two-year results from a comparative effectiveness randomized clinical trial. Ophthalmology. 2016;123:1351-9.

11. Elman M, Ayala A, Bressler N, Browning D, Flaxel C, Glassman A, et al Intravitreal ranibizumab for diabetic macular edema with prompt versus deferred laser treatment: 5-year randomized trial results. Ophthalmology. 2015;122:375-81.

12. Bressler S, Qin H, Melia M, Bressler N, Beck R, Chan C, et al. Exploratory analysis of the effect of intravitreal ranibizumab or triamcinolone on worsening of diabetic retinopathy in a randomized clinical trial. JAMA Ophthalmol. 2013;131:1033-40.

13. Brown D, Schmidt U, Do D, Holz F, Boyer D, Midena E, et al. Intravitreal aflibercept for diabetic macular edema: 100-week results from the VISTA and VIVID studies. Ophthalmology. 2015;122:2044-52.

14. Nguyen Q, Brown D, Marcus D, Boyer D, Patel S, Feiner L, et al. Ranibizumab for diabetic macular edema: results from 2 phase-III randomized trials: RISE and RIDE. Ophthalmology. 2012;119:789-801.

15. Gross J, Glassman A, Jampol L, Inusah S, Aiello L, Antoszyk A, et al. Panretinal photocoagulation vs. intravitreous ranibizumab for proliferative diabetic retinopathy: a randomized clinical trial. JAMA. 2015;314:2137-46.

16. Sivaprasad S, Prevost A, Vasconcelos J, Riddell A, Murphy C, Kelly J, et al. Clinical efficacy of intravitreal aflibercept versus panretinal photocoagulation for best corrected visual acuity in patients with proliferative diabetic retinopathy at 52 weeks (CLARITY): a multicentre, single-blinded, randomised, controlled, phase $2 \mathrm{~b}$, non-inferiority trial. Lancet. 2017:389:2193-203.

17. Gross J, Glassman A, Liu D, Sun J, Antonszyk A, Baker C, et al. Five-year outcomes of panretinal photocoagulation vs. intravitreous ranibizumab for proliferative diabetic retinopathy: a randomized clinical trial. JAMA Ophthalmol. 2018;136:1138-48.

18. Bressler S, Liu D, Glassman A, Blodi B, Castellarin A, Jampol L, et al. Change in diabetic retinopathy through 2 years: secondary analysis of a randomized clinical trial comparing aflibercept, bevacizumab and ranibizumab. JAMA Ophthalmol. 2017;135:558-68.

19. Silva $P$, De la Cruz A, Ledesmav M, Hemert J, Radwa A, Cavallerano J, et al. Diabetic retinopathy severity and peripheral lesions are associated with nonperfusion on ultrawide field angiography. Ophthalmology. 2015;122:2465-72

20. Bressler S, Beaulieu W, Glassman A, Gross J, Jampol L, Melia M, et al. Factors associated with worsening proliferative diabetic retinopathy in eyes treated with panretinal photocoagulation or ranibizumab. Ophthalmology. 2017:124:431-9.

21. Couturier A, Rey P, Erginay A, Lavia C, Bonnin S, Dupas B, et al. Widefield OCT-angiography and fluorescein angiography assessments of nonperfusion in diabetic retinopathy and edema treated with anti-vascular endothelial growth factor. Ophthalmology. 2019;126:1685-94.

22. Bonnin S, Dupas B, Lavia C, Erginay A, Dhundass M, Couturier A, et al. Anti-vascular endothelial growth factor therapy can improve diabetic retinopathy score without change in retinal perfusion. Retina. 2019;39:426-34.

23. Sun J, Glassman A, Beaulieu W, Stockdale C, Bressler N, Flaxel C, et al. Rationale and application of the Protocol S anti-vascular endothelial growth factor algorithm for proliferative diabetic retinopathy. Ophthalmology. 2019;126:87-95.

24. Ross E, Hutton D, Stein J, Bressler N, Jampol L, Glassman A, et al. Cost-effectiveness of aflibercept, bevacizumab, and ranibizumab for diabetic macular edema treatment: analysis from the diabetic retinopathy clinical research network comparative effectiveness trial. JAMA Ophthalmol. 2016;134:888-96

25. Hooper P, Boucher M, Colleaux K, Cruess A, Greve M, Lam W, et al. Contemporary management of diabetic retinopathy in Canada: from guidelines to algorithm guidance. Ophthalmologica. 2014;231:2-15.

26. Bandello F, Cunha J, Chong N, Lang G, Massin P, Mitchell P, et al. New approaches for the treatment of diabetic macular oedema: recommendations by an expert panel. Eye (Lond). 2012;26:485-93.

27. Mitchell $P$, Wong $T$. Management paradigms for diabetic macular edema. Am J Ophthalmol. 2014;157:505-13.

28. Elman M, Aiello L, Beck R, Bressler N, Bressler S, Edwards A, et al. Randomized trial evaluating ranibizumab plus prompt or deferred laser or triamcinolone plus prompt laser for diabetic macular edema. Ophthalmology. 2010;117:1064-77.

29. Payne J, Wykoff C, Clark W, Bruce B, Boyer D, Brown M. Long-term outcomes of treat-and-extend ranibizumab with and without navigated laser for diabetic macular oedema: TREX-DME 3-year results. $\mathrm{Br} \mathrm{J}$ Ophthalmol. 2020;105:253-7.

30. Lozano E, González J, Olvera A, Treviño E, Rodríguez J, Mohamed K. Short-term outcomes after the loading phase of intravitreal bevacizumab and subthreshold macular laser in non-center involved diabetic macular edema. Int J Ophthalmol. 2018;11:981-5.

31. Bressler S, Beaulieu W, Glassman A, Gross J, Melia M, Chen E, et al. Photocoagulation versus ranibizumab for proliferative diabetic retinopathy: should baseline characteristics affect choice of treatment? Retina. 2019;39:1646-54.

32. Diabetic Retinopathy Clinical Research Network. Randomized clinical trial evaluating intravitreal ranibizumab or saline for vitreous hemorrhage from proliferative diabetic retinopathy. JAMA Ophthalmol. 2013;131:283-93 
33. Hutton D, Stein J, Bressler N, Jampol L, Browning D, Glassman A Cost-effectiveness of intravitreous ranibizumab compared with panretinal photocoagulation for proliferative diabetic retinopathy secondary analysis from a Diabetic Retinopathy Clinical Research Network Randomized Clinical Trial. JAMA Ophthalmol. 2017;135:576-84.

34. Sebag J. Anatomy and pathology of the vitreo-retinal interface. Eye. 1992:6:541-52.

35. Nasrallah F, Jalkh A, Van Coppenolle F, Trempe C, McMeel J, Schempens $C$. The role of the vitreous in diabetic macular edema. Ophthalmology. 1988;95:1335-9.

36. Ophir A, Martinez M, Mosqueda $P$, Trevino A. Vitreous traction and epiretinal membranes in diabetic macular oedema using spectral-domain optical coherence tomography. Eye (Lond). 2010;24:1545-53.

37. Ophir A, Martinez M. Epiretinal membranes and incomplete posterior vitreous detachment in diabetic macular edema, detected by spectral-domain optical coherence tomography. Invest Ophthalmol Vis Sci. 2011;52:6414-20.
38. Gunduz K, Bakri S. Management of proliferative diabetic retinopathy. Compr Ophthalmol Update. 2007;8:245-56.

39. Chu T, Lopez P, Cano M, Freeman W, Lean J, Liggett P, et al. Posterior vitreoschisis: an echographic finding in proliferative diabetic retinopathy. Ophthalmology. 1996;103:315-22.

40. Gaucher D, Tadayoni R, Erginay A, Haouchine B, Gaudric A, Massin P. Optical coherence tomography assessment of the vitreoretinal relationship in diabetic macular edema. Am J Ophthalmol. 2005;139:807-13.

41. Lin J, Chang J, Yannuzzi N, Smiddy W. Cost evaluation of early vitrectomy versus panretinal photocoagulation and intravitreal ranibizumab for proliferative diabetic retinopathy. Ophthalmology. 2018;125:1393-400

42. Wykoff C; PANORAMA investigators. Intravitreal aflibercept for moderately severe to severe non-proliferative diabetic retinopathy (NPDR). The phase 3 PANORAMA study. Disponible en: https://investor.regeneron. com/static-files/89add496-a979-4c71-80b5-a8aebab7d95d. 


\title{
Edema macular diabético y catarata
}

\author{
Carla Pérez-Montaño* \\ Hospital de Nuestra Señora de la Luz, Ciudad de México, México
}

Debido a la mayor expectativa de vida y el envejecimiento de la población, las cataratas son la principal causa de ceguera en el mundo. La Organización Mundial de la Salud predice que las cataratas causarán una pérdida visual de moderada a grave en 57.1 millones de personas en el año $2020^{1}$.

La diabetes es una enfermedad crónica que afecta a uno de cada ocho adultos en todo el mundo. Los pacientes con diabetes tienen un riesgo elevado de desarrollar cataratas visualmente significativas en comparación con sujetos controles equiparados en edad sin diabetes, con una razón de momios de 1.97 (intervalo de confianza del 95\% [IC95\%]: $0.951-2.577 ; p<$ $0.001)$. Las cataratas en pacientes diabéticos se desarrollan hasta 20 años antes y maduran rápidamente con alteración visual que requiere extracción quirúrgica temprana ${ }^{1}$.

La formación de una catarata densa puede enmascarar la gravedad de una retinopatía diabética y dificultar el tratamiento adecuado con fotocoagulación láser. La extracción de la catarata permite la mejor visualización de la mácula y la retina, facilitando el tratamiento con láser y la realización de estudios de imagen, así como el monitoreo de la enfermedad ${ }^{1}$.

Con las técnicas modernas de facoemulsificación, los tiempos quirúrgicos y las complicaciones han disminuido, y los resultados visuales han mejorado, pero la diabetes se asocia con frecuencia a un incremento en el riesgo de aparición y progresión de edema macular, ya que esta cirugía, incluso en su forma moderna microincisional, es un procedimiento invasivo intraocular asociado a liberación de citocinas proinflamatorias. El incremento en la inflamación y la rotura de la barrera hematorretiniana pueden desempeñar un papel en el desarrollo de edema macular después de la cirugía de catarata. Apoyando esto, se ha demostrado que existe un incremento del factor de crecimiento endotelial vascular (VEGF, vascular endothelial growth factor) dentro del primer mes posterior a la cirugía. Los mediadores angiogénicos e inflamatorios liberados tras la cirugía pueden incrementar la permeabilidad de los capilares de la retina y causar exacerbación del edema macular. Por consiguiente, la probabilidad de que esto pueda desarrollarse en ojos que ya tienen comprometida la barrera hematorretiniana debido a retinopatía diabética preexistente puede ser aún mayor ${ }^{1,2}$.

En los ojos de pacientes con diabetes, dos afecciones con una evolución natural diferente pueden contribuir a la formación de edema macular después de la cirugía: el empeoramiento de un edema macular preexistente y la aparición de edema macular cistoideo pseudofáquico $(\mathrm{EMCP})^{3}$.

La aparición de edema macular después de la cirugía, en ojos sin evidencia de edema macular previo, tiene una evolución natural relativamente benigna, con una incidencia pico a las 5-6 semanas y resolución espontánea dentro de 6 meses en más del $68 \%$ de los ojos. En caso de empeoramiento de un edema macular preexistente, el curso natural es más grave, con tendencia a progresar ${ }^{3}$.

La diferenciación entre edema macular diabético (EMD) y EMCP puede ser desafiante, ya que comparten características. Los dos edemas presentan acumulación de fluido dentro del tejido retiniano en la región macular. Para tratar de manera adecuada el edema macular es crucial clasificarlo correctamente, ya que el tratamiento puede variar dependiendo de la 
condición subyacente. Un estudio describió los criterios de tomografía de coherencia óptica para diferenciar el EMCP del EMD. Un grosor macular central (GMC) más alto/proporción de volumen retiniano, una capa nuclear externa (CNE) más gruesa/capa de Henle, espacios cistoideos en la capa nuclear interna (CNI), ausencia de membrana epirretiniana, bandas hiperreflectivas externas intactas y presencia de líquido subretiniano indican EMCP. En contraste, una proporción de grosor mayor de $\mathrm{CNE} / \mathrm{CNI}$, la presencia de microaneurismas, exudados duros, microfoci, espacios cistoideos principalmente en la CNE y disrupción de las capas de fotorreceptores son características del EMD (figura 1) ${ }^{3}$.

Existe desacuerdo en las observaciones de varios estudios en cuanto al incremento del GMC o el desarrollo de edema macular posterior a cirugía de catarata en pacientes diabéticos.

Sze et al. ${ }^{4}$ en 2012, reportaron que los resultados visuales a los 12 meses de la cirugía de catarata en pacientes diabéticos sin retinopatía y con retinopatía diabética, pero sin fotocoagulación previa, fueron iguales que los obtenidos en pacientes no diabéticos, con una mejoría en promedio de 2 líneas de visión. Guliani, et al. ${ }^{5}$ valoraron de forma prospectiva los cambios en el GMC posterior a cirugía de catarata en diabéticos bien controlados (hemoglobina glucosilada $[\mathrm{HbA1c}]<7 \%)$ sin retinopatía diabética (330 pacientes) y no diabéticos (330 pacientes), con un seguimiento de 6 semanas. Aunque no hubo diferencia significativa en el GMC entre los dos grupos, sí hubo un incremento significativo en el GMC al final de la primera y la sexta semanas posquirúrgicas en comparación con los valores preoperatorios. El grosor disminuyó a valores prequirúrgicos hasta el final de la sexta semana. Este estudio demuestra la influencia de la cirugía de catarata no complicada en el GMC en diabéticos con buen control sin retinopatía, y que no varía en comparación con la respuesta presentada por personas sanas no diabéticas ${ }^{1,5}$.

En un estudio de casos y controles realizado por Sahin, et al. ${ }^{6}$ en 4500 pacientes diabéticos sin edema macular preoperatorio, la incidencia de edema macular posquirúrgico fue del $4 \%$, la cual es mayor que en la población sin diabetes $(p<0.001)$.

Por otra parte, Katsimpris, et al ${ }^{7}$. encontraron un aumento del GMC después de la cirugía de catarata en pacientes diabéticos sin retinopatía diabética en comparación con sus valores preoperatorios y con un grupo control, con seguimiento a 12 meses. Los ojos de los pacientes diabéticos sin retinopatía diabética presentaron mayor GMC y mayor incidencia de edema macular cistoideo posquirúrgico que los ojos de los controles sanos.

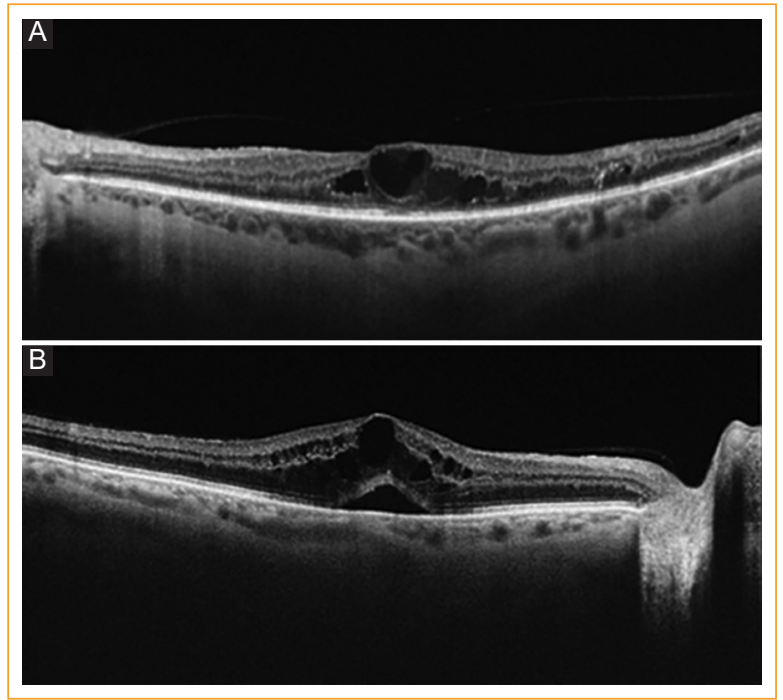

Figura 1. A: edema macular diabético: espacios cistoideos principalmente en capa nuclear externa/capa de Henle, exudados duros y disrupción de las capas de fotorreceptores. B: edema macular cistoideo pseudofáquico: fluido subretiniano y bandas hiperreflectivas externas intactas.

\section{Edema macular cistoideo pseudofáquico}

El desarrollo de EMCP es la causa más frecuente de alteración visual posterior a cirugía de catarata en la población general. En estudios antiguos, la incidencia de EMCP variaba entre el $0.2 \%$ y el $20 \%$; en estudios más recientes se reporta una tasa menor, que oscila desde menos del $1 \%$ hasta el $2-3 \%$, pero alcanza el $4 \%$ en la población diabética y llega al 10-12\% cuando se contabilizan los pacientes con retinopatía diabética no proliferativa grave y proliferativa. La tasa de detección de EMCP varía significantemente según el método, ya sea angiografía con fluoresceína o tomografía de coherencia óptica; ambos son métodos más sensibles y con mayores tasas de detección comparados con la clínica'.

Un edema macular diabético preexistente representa el principal factor de riesgo para su deterioro después de la cirugía de catarata no complicada, con una incidencia de EMCP del $16.3 \%$ en pacientes con EMD previo ${ }^{3}$.

Se cree que la fisiopatología del EMCP en los pacientes diabéticos se debe a una respuesta inflamatoria posoperatoria. El trauma físico asociado a la cirugía, incluyendo la disrupción de la barrera hematorretiniana, puede inducir una respuesta inflamatoria y la liberación de mediadores, como prostaglandinas y leucotrienos desde el ácido araquidónico. Las prostaglandinas son liberadas naturalmente desde el iris y el cuerpo ciliar, y migran a la retina después de la cirugía de catarata. La respuesta inflamatoria puede 


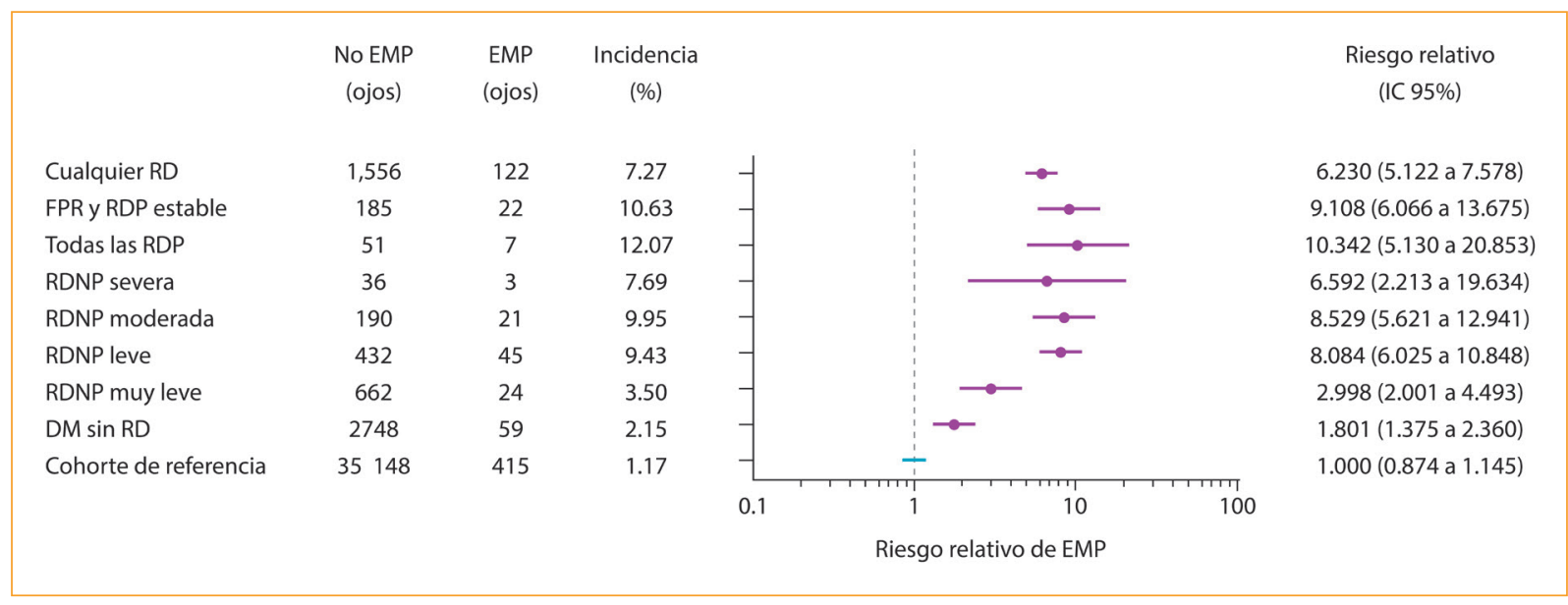

Figura 2. Riesgo relativo para nuevo edema macular según el grado de retinopatía diabética (RD). Solo se incluyeron pacientes sin maculopatía preoperatoria. DM: diabetes mellitus; EMCP: edema macular cistoideo pseudofáquico; IC 95\%: intervalo de confianza del 95\%; FPR: fotocoagulación; RDNP: retinopatía diabética no proliferativa; RDP: retinopatía diabética proliferativa;

conducir a la activación de la cascada inmunitaria, involucrando neutrófilos, macrófagos, linfocitos $T$ y mediadores inflamatorios. En los diabéticos, la barrera hematorretiniana se encuentra dañada en un grado variable y es causa del EMCP, dependiendo de muchos factores, incluyendo la duración y la gravedad de la enfermedad, la presencia de retinopatía y los tratamientos previos con fotocoagulación o inyección de esteroides. Se ha reportado que la incidencia es mayor en la diabetes tipo 2 no dependiente de insulina que en la dependiente de insulina ${ }^{3}$.

Chu, et al. ${ }^{8}$ reportaron que el riesgo relativo de EMCP estaba asociado al estadio de la retinopatía diabética, el cual fue de 1.8 (IC95\%: 1.36-2.36) en pacientes diabéticos sin signos de retinopatía, de 6.23 (IC95\%: 5.12-7.58) en pacientes con retinopatía diabética no proliferativa y de 10.34 (IC95\%: 5.13$20.85)$ en pacientes con retinopatía diabética proliferativa (figura 2). Los riesgos relativos medios comparados con la cohorte de referencia están trazados con su IC95\%. Los grupos que alcanzan un valor estadísticamente significativo de al menos $p<0.05$ están marcados en rojo.

En un estudio llevado a cabo por Yang, et al. ${ }^{9}$, la duración, la gravedad, el tipo de diabetes, la dureza del cristalino y los valores de $\mathrm{HbA} 1 \mathrm{c}$ fueron factores de riesgo para el desarrollo de EMCP en pacientes diabéticos. Tales hallazgos indican que estos pacientes deben recibir profilaxis dependiendo del estadio de la retinopatía diabética.

\section{Edema macular diabético}

Además de un mayor riesgo de EMCP, los pacientes diabéticos con edema macular preexistente tienen más riesgo de empeorar el edema tras la cirugía de catarata. En el Early Treatment Diabetic Retinopathy Study (ETDRS), reporte 25, la presencia de edema macular clínicamente significativo preexistente, aunque no fue significativamente más prevalente después de la cirugía (31\% frente a $29 \%$ ), se asoció con peores resultados visuales".

Dowler, et al. ${ }^{10}$, en su estudio de 32 pacientes diabéticos a quienes se realizó cirugía de catarata, reportaron que la presencia de edema macular clínicamente significativo preoperatorio hacía que el edema no resolviera espontáneamente en los 12 meses de seguimiento, mientras que sí se resolvieron el $69 \%$ de los nuevos casos de edema surgidos después de la cirugía ( $p=0.05$ ). Además, el edema macular clínicamente significativo en el momento de la cirugía de catarata se asoció a peor resultado visual al año $(p=0.005)$.

El DRCR.net, en su protocolo $Q$, reportó los resultados de la cirugía de catarata en pacientes sin involucro central definitivo de EMD. Identificaron que la progresión o el desarrollo de edema macular en el subcampo central podía estar influenciado por la presencia de EMD preexistente y su tratamiento, ya que hasta el $20 \%$ de los ojos con antecedente de tratamiento tenían edema con involucro central, frente al $4 \%$ de los ojos sin tratamiento previo $(p<0.001)^{8}$. 


\section{Prevención del edema macular cistoideo pseudofáquico en pacientes diabéticos sin edema macular preexistente}

\section{Antiinflamatorios no esteroideos}

Como muchos casos de EMCP son leves y suelen resolverse espontáneamente, la evaluación precisa de los beneficios clínicos de los fármacos antiinflamatorios no esteroides (AINE) disponibles es desafiante ${ }^{3}$.

Varios estudios han mostrado que los AINE, solos o en combinación con esteroides, reducen significativamente el riesgo de desarrollar EMCP en pacientes no diabéticos, en comparación con los corticosteroides tópicos. Los antiinflamatorios tópicos son efectivos principalmente al inhibir la vía de la ciclooxigenasa 2 y bloquear la producción de prostaglandinas; de este modo se reducen la incidencia, la gravedad y la duración del edema macular ${ }^{3}$.

Una serie de estudios clínicos han evaluado la eficacia de los AINE en la población diabética. En 2017 se estudiaron retrospectivamente 89,731 ojos y se observó una prevalencia de EMCP del $1.3 \%$ en ojos tratados con terapia tópica con AINE (ketorolaco) más esteroides, frente al $1.7 \%$ en el grupo de monoterapia con esteroides tópicos. El uso de AINE se asoció con una menor incidencia de EMCP en los pacientes sin diabetes y con diabetes sin retinopatía ${ }^{3}$.

El uso tópico de diclofenaco al $0.1 \%$ en pacientes con retinopatía diabética, cuatro veces al día por 7 días antes de la cirugía de catarata y los 30 días posteriores, resultó en una concentración intraocular significativamente más baja de interleucina $2(p=0.007)$ y un menor incremento del GMC ( $p<0.001)$, en comparación con la terapia estándar con dexametasona al $0.1 \%$ tópica cuatro veces al día por 30 días $^{3}$.

Diferentes estudios han reportado una eficacia variable del bromfenaco en pacientes con retinopatía diabética no proliferativa en comparación con esteroides tópicos para la prevención del EMCP. En un estudio prospectivo, aleatorizado, que comparó bromfenaco al $0.9 \%$ y acetónido de prednisolona al 1\% (22 ojos por grupo de estudio), no hubo diferencias significativas en el GMC entre los tratamientos, a 1 semana, 1 mes y 2 meses después de la cirugía. En otro estudio prospectivo llevado a cabo por Terada, et al..$^{12} 51$ pacientes operados de cirugía de catarata fueron asignados aleatoriamente a tratamiento posquirúrgico con bromfenaco al $0.1 \%$ como monoterapia por 8 semanas o tratamiento combinado de bromfenaco al $0.1 \%$ por 8 semanas y betametasona al $0.1 \%$ por 2 semanas. El GMC aumentó significativamente en los pacientes en monoterapia con bromfenaco al mes de la cirugía. Al final del estudio no hubo diferencias en la agudeza visual.

El nepafenaco, a diferencia de los AINE convencionales, es un profármaco con permeabilidad potencializada. Su rápida bioactivación a amfenaco por las hidrolasas intraoculares dentro de los tejidos oculares (epitelio del cuerpo ciliar, retina, coroides y cornea) resulta en un fármaco activo y específico suministrado a los segmentos anterior y posterior ${ }^{3}$.

Singh, et al. ${ }^{13}$ dirigieron dos estudios prospectivos, aleatorizados, doble ciego, de fase 3 , que compararon los efectos del nepafenaco $0.3 \%$ tópico frente a solo vehículo en pacientes con retinopatía diabética a los que se realizó una extracción de catarata. Un total de 615 pacientes en el grupo 1 y de 605 en el grupo 2 se aleatorizaron para recibir nepafenaco $0.3 \%$ una vez al día previamente a la cirugía y los 90 días posteriores o solo vehículo. Todos los pacientes recibieron acetónido de prednisolona por 4 semanas tras la cirugía. En el estudio 1, el grupo con nepafenaco presentó menos edema macular posquirúrgico (2.3\% frente a $17.3 \%$; $p$ $<0.001$ ), y en el estudio 2 los resultados fueron similares $(5.9 \%$ frente a $14.3 \%)$. Un mayor porcentaje de pacientes en el grupo de nepafenaco mejoraron su agudeza visual a más de 15 letras.

Aun con la evidencia actual, falta claridad sobre qué AINE podría ser el más efectivo. El bromfenaco y el nepafenaco tienen ventajas farmacocinéticas, ya que ambas moléculas pueden penetrar rápidamente la barrera corneal y alcanzar concentraciones terapéuticas tempranas y sostenidas. Además, han mostrado una reducción eficaz de la incidencia de EMCP en estudios clínicos aleatorizados, por lo que deben considerarse como la primera opción de tratamiento ${ }^{3}$.

\section{Esteroides tópicos}

Constituyen una terapia eficaz para suprimir la inflamación posquirúrgica. Comparados con los AINE, sus efectos son inferiores para reducir la incidencia de EMCP, lo que podría explicarse por su menor penetración en el ojo al ser de uso tópico. Los esteroides pueden causar más efectos adversos que los AINE, como aumento de la presión intraocular, retraso en la curación de heridas y reactivación de infecciones por herpes, lo que limita su uso prolongado ${ }^{3}$. 


\section{Prevención del edema macular cistoideo pseudofáquico en pacientes diabéticos sin edema macular diabético}

\section{Anti-VEGF y esteroides intravítreos}

Debido a que los estudios indican un papel clave del VEGF en la patogénesis del EMCP, las inyecciones intravítreas de anti-VEGF han generado un gran interés. Los estudios han evaluado el efecto de anti-VEGF intravitreos al final de la cirugía de catarata en diabéticos sin edema macular clínicamente significativo, y reportan una disminución del GMC, una menor incidencia de edema macular cistoideo y alguna mejoría de la agudeza visual mejor corregida (AVMC) al final del seguimiento. También se ha evaluado el papel de los esteroides inyectados subconjuntivalmente $o$ intravítreos en pacientes diabéticos sin edema macular. El acetónido de triamcinolona es un potente corticosteroide que puede reducir la rotura de la barrera hematorretiniana, inhibir la producción de prostaglandinas y disminuir la expresión de VEGF, y también ha mostrado menor grosor macular, menor incidencia de edema macular cistoideo en ojos tratados al final de la cirugía de catarata y efectos que pueden durar hasta 6 meses $^{3}$.

A pesar de los teóricos beneficios de los anti-VEGF y los esteroides intravítreos en la prevención del EMCP, aún falta evidencia de alta calidad para recomendar estos tratamientos sistemáticamente. Por otra parte, la administración intravítrea puede condicionar un riesgo de endoftalmitis y desprendimiento de retina en ojos que no presentaban edema macular antes de la cirugía ${ }^{3}$.

La Sociedad Europea de Cirujanos de Catarata y Refractiva respaldó el estudio multicéntrico PREMED (prevención del edema macular después de cirugía de catarata) a 2 años, doble ciego y aleatorizado, para proveer pautas basadas en la evidencia para la prevención del EMCP y su tratamiento en pacientes con y sin diabetes. En la población diabética (reporte 2) se aleatorizaron cuatro grupos, que recibieron:

- Grupo 1: régimen estándar (reporte 1) de bromfenaco $0.09 \%$ dos veces al día (2 días previos al evento quirúrgico y 2 semanas posteriores) y fosfato de dexametasona $0.1 \%$ cuatro veces al día ( 2 días previos al evento y 1 semana posterior, después 1 gota menos al día cada semana).

- Grupo 2: bromfenaco 0.09\%, dexametasona 0.1 e inyección subconjuntival de $40 \mathrm{mg}$ de triamcinolona transquirúrgica.

- Grupo 3: bromfenaco 0.09\%, dexametasona 0.1 e inyección intravítrea de $1.25 \mathrm{mg}$ de bevacizumab transquirúrgica.
- Grupo 4: bromfenaco 0.09\%, dexametasona 0.1, inyección subconjuntival de $40 \mathrm{mg}$ de triamcinolona transquirúrgica e inyección intravítrea de $1.25 \mathrm{mg}$ de bevacizumab transquirúrgica.

Una sola inyección subconjuntival de triamcinolona previno efectivamente el desarrollo de EMCP en pacientes diabéticos (incidencia del $0 \%$ a 6 y 12 meses). Sin embargo, el riesgo de desarrollar edema cistoideo debe balancearse frente al riesgo de incremento en la presión intraocular, ya que el $7.1 \%$ de los pacientes tratados con $40 \mathrm{mg}$ de triamcinolona tuvieron una presión intraocular de $25 \mathrm{mmHg}$ o más en las siguientes 12 semanas. El bevacizumab intravítreo no mostró un efecto significativo en la prevención del EMCP ${ }^{3,14}$.

\section{Estrategias para evitar el empeoramiento del edema macular diabético preexistente después de la cirugía de catarata}

Los resultados de estudios como el Early Treatment Diabetic Retinopathy Study (ETDRS) $)^{15}$ proveen evidencia de que la fotocoagulación debe ser considerada antes de la cirugía de catarata cuando sea posible. De los 3711 pacientes incluidos en el estudio, la cirugía se realizó en 270 ojos, y dos tercios de ellos habían recibido fotocoagulación focal para el edema macular antes de la cirugía. No hubo diferencia significativa en la proporción de ojos con EMD antes y después de la extracción de la catarata. Un año después de la cirugía de catarata, la proporción de ojos con una mejoría de agudeza visual de 2 o más líneas fue del $64 \%$ en el grupo de láser temprano y del $49 \%$ en el grupo de láser diferido. Los ojos asignados a fotocoagulación temprana mostraron una tendencia hacia una mejor agudeza visual (estadísticamente no significativa) ${ }^{3}$.

Los resultados del ETDRS también aportan evidencia de que la fotocoagulación panretiniana debe realizarse antes de la cirugía de catarata, ya que previene la progresión de la retinopatía diabética causada por la cirugía. Sin embargo, este estudio fue realizado en 1980, y desde entonces ha habido muchos cambios en las técnicas y las plataformas quirúrgicas. Reportes recientes indican que debe evitarse realizar la fotocoagulación panretiniana antes de la cirugía de catarata en pacientes con retinopatía diabética no proliferativa grave o proliferativa temprana, debido a que puede incrementar el riesgo de progresión posquirúrgica del edema macular. En un estudio prospectivo, 58 ojos con cataratas similares y retinopatía diabética no proliferativa grave y proliferativa temprana fueron asignados aleatoriamente a tratamiento con láser posterior 0 


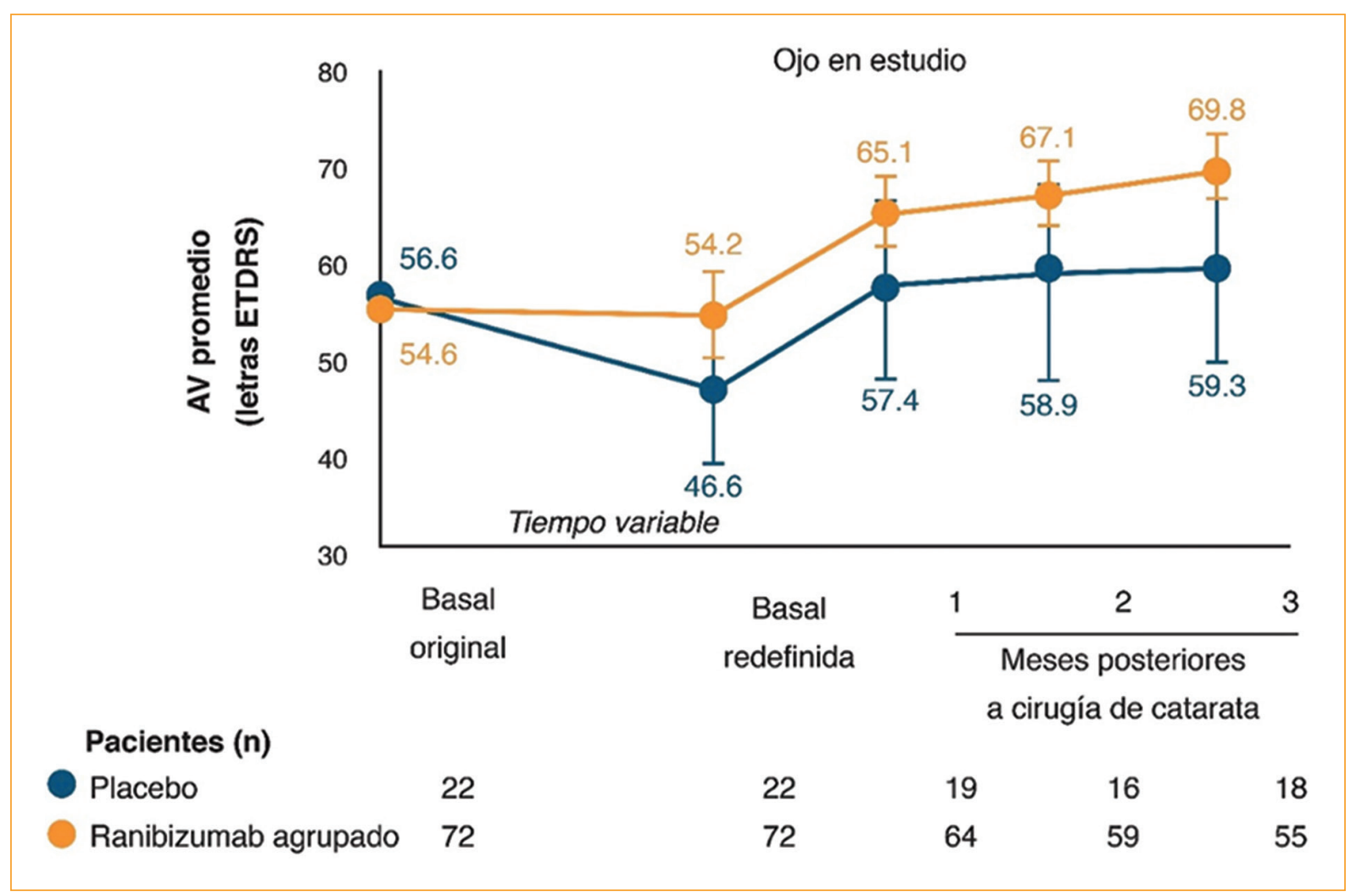

Figura 3. Agudeza visual (AV) antes y después de la cirugía de catarata en ojos fáquicos en los grupos tratados con ranibizumab $(0.3$ y $0.5 \mathrm{mg})$ o placebo.

previo a la cirugía de catarata. El porcentaje de ojos con una agudeza visual de 20/40 o mejor fue significativamente más alto en el grupo de cirugía previa a la fotocoagulación panretiniana $(96.6 \%$ frente a $69 \%$; $p<0.012)$. La tasa de progresión del edema macular disminuyó significativamente en ese mismo grupo ${ }^{3}$.

La administración intravítrea de agentes anti-VEGF y esteroides combinados con cirugía de catarata ha sido un campo de estudio creciente para mejorar la agudeza visual y prevenir el empeoramiento del edema en pacientes con EMD preexistente y cataratas incapacitantes.

No existe tratamiento ni momento universalmente aceptados para los pacientes con EMD preexistente en el periodo perioperatorio. Una opción es pretratar el EMD con anti-VEGF y esteroides antes de la remoción de la catarata para lograr efectos protectores, y otra es hacer simultáneamente la cirugía con la intervención farmacológica ${ }^{16}$.

\section{Tratamiento previo a la cirugía}

Los datos obtenidos de los estudios RISE/RIDE (figura 3) revelaron que la cirugía de catarata no afecta negativamente la recuperación visual en los pacientes tratados con ranibizumab intravítreo; más bien, los pacientes ganaron en promedio 2 líneas de visión desde la visita previa a la cirugía al mes posquirúrgico. Los pacientes esperaron 7 días después de su más reciente aplicación de antiVEGF para operarse y reiniciaron el tratamiento antiVEGF 4 semanas tras la facoemulsificación. Los resultados fueron similares en los estudios VIVID/VISTA en pacientes operados de facoemulsificación ${ }^{3,9}$.

El implante intravítreo de dexametasona de $0.7 \mathrm{mg}$ es eficaz y seguro para el tratamiento del EMD. Sin embargo, las cataratas fueron un evento adverso común, con una tasa en ojos fáquicos del $67.9 \%$ a 3 años de seguimiento. Un subanálisis del estudio MEAD ${ }^{17}$ reveló que los pacientes sometidos a cirugía de catarata durante el estudio ganaron el mismo beneficio visual que los pacientes pseudofáquicos (+6.7 frente $a+6.5$ letras ETDRS) 3 .

\section{Tratamiento simultáneo con la cirugía de catarata}

Un segundo enfoque es combinar la extracción de catarata con la inyección farmacológica de medicamentos. El uso no aprobado de bevacizumab 
intravítreo combinado con la cirugía de catarata en pacientes diabéticos con antecedente de edema macular ha sido evaluado en varios estudios. En el estudio llevado a cabo por Takamura, et al. ${ }^{18}$, se asignó a 42 pacientes para recibir de forma aleatoria bevacizumab intravítreo en combinación con cirugía (grupo de tratamiento) o solo cirugía (grupo control), en los meses 1 y 3 el GMC medio disminuyó significativamente en el grupo de tratamiento. La agudeza visual mejoró también de manera significativa en los dos grupos a los 3 meses de seguimiento, pero los pacientes tratados con bevacizumab mostraron mejor agudeza visual de manera significativa $(p=0.034)^{3}$.

Lanzagorta-Aresti, et al. ${ }^{19}$ reportaron que, en los pacientes tratados con bevacizumab intravítreo combinado con facoemulsificación, el GMC medio permaneció estable durante 6 meses de seguimiento y la agudeza visual mejoró significativamente a los 3 y 6 meses ( $p=0.048$ y $p=0.035$, respectivamente).

Los esteroides intravítreos en el momento de la cirugía de catarata se han propuesto para el tratamiento de ojos con EMD preexistente que se someterán a cirugía de catarata. Lam, et al. ${ }^{20}$ investigaron el uso de $4 \mathrm{mg}$ de acetónido de triamcinolona intravítreo combinado con cirugía en 17 ojos con EMD en un estudio prospectivo y no comparativo. A los 6 meses, la agudeza visual mejoró significativamente $(p=0.015)$. La reducción media del GMC después de la cirugía fue estadísticamente significativa en todos los intervalos analizados hasta los 6 meses de seguimiento.

Takata, et al. ${ }^{21}$ compararon la administración intravítrea $(4 \mathrm{mg})$ y subtenoniana $(40 \mathrm{mg})$ de acetónido de triamcinolona durante la facoemulsificación en 19 pacientes con EMD refractario. La agudeza visual mejoró y el GMC disminuyó, ambos significativamente, a las 4, 8 y 12 semanas de la cirugía en los pacientes tratados con esteroide intravítreo.

Un estudio prospectivo llevado a cabo por Lim, et al. ${ }^{22}$ se comparó el uso de bevacizumab intravítreo frente a triamcinolona intravítrea en el tratamiento del EMD en el momento de la extracción de catarata. Se reportó que las dos terapias eran igual de eficaces para mejorar la AVMC, pero que solo la triamcinolona se asociaba a una reducción mantenida del GMC.

Sze, et al..$^{23}$ analizaron retrospectivamente 12 ojos con EMD tratados con implante de dexametasona en el momento de la cirugía de catarata. Los resultados mostraron una reducción significativa en el GMC y una ganancia visual de al menos 2 líneas (ETDRS) en el $66.7 \%$ de los ojos. La eficacia del implante de dexametasona combinado con cirugía de catarata fue también confirmada por
Agarwal, et al., ${ }^{24}$ en un estudio prospectivo, controlado y aleatorizado, en el cual la agudeza visual mejoró y el GMC disminuyó significativamente, a las 6, 12 y 24 semanas, en comparación con el grupo control.

La capacidad del implante intravítreo de dexametasona para atenuar el empeoramiento del EMD después de la facoemulsificación fue estudiada de forma prospectiva por Panozao, et al. ${ }^{25}$ en 11 ojos con EMD naïve y 8 ojos con edema refractario. La mejoría visual se consolidó al mes, con una mejoría media de 18 letras. El edema refractario mostró el mismo beneficio, pero recurrió más pronto (4 frente a 5.8 meses; $p<0.01$ ).

De acuerdo con lo anterior, se puede concluir que la coexistencia de catarata y diabetes constituye un factor principal de riesgo para limitar la recuperación visual después de una microcirugía exitosa. La terapia tópica con AINE, debido a su poca invasión, alta eficacia y baja tasa de complicaciones, es actualmente de elección para prevenir el EMCP. El uso profiláctico de anti-VEGF y esteroides intravítreos en diabéticos sin edema macular, aun siendo eficaz, conlleva el riesgo de posibles efectos adversos. Por el contrario, en los pacientes con EMD en el momento de la cirugía, la terapia intravítrea con anti-VEGF o esteroides ayuda a prevenir el deterioro del GMC y de la agudeza visual. La disponibilidad de agentes anti-VEGF y de sistemas de liberación de esteroides ha revolucionado el manejo del EMD. Al usarse en combinación con cirugía de catarata, pueden ayudar a tratar un edema preexistente y prevenir su empeoramiento. Los esteroides intravítreos, a pesar de su riesgo de incrementar la presión intraocular, presentan ventajas sobre los anti-VEGF debido a su actividad prolongada y mejor control del GMC con menor número de inyecciones. Combinar las modernas técnicas de facoemulsificación con intervenciones farmacológicas personalizadas brinda al paciente con diabetes los mejores resultados posibles visuales y anatómicos.

\section{Bibliografía} diabetes: management strategies. Sem Ophthalmol. 2018;33:75-82.

2. Moshfeghi A, Shapiro H, Lemmon L, Gune S. Impact of cataract surgery during treatment with ranibizumab in patients with diabetic macular edema. Ophthalmol Retina. 2018:2:86-90.

3. Sarao V, Veritti D, Manutto E, Rasu N, Borrelli E, Loewenstein A, et al. Pharmacotherapeutic management of macular edema in diabetic subjects undergoing cataract surgery. Expert Opin Pharmacother. 2018;19: 1551-63.

4. Sze C, Mitchell P, Rochtchina E, Loryn T, Hong T, Jin J. Visual outcomes 12 months after phacoemulsification cataract surgery in patients with diabetes. Acta Ophthalmol. 2012:90:173-78.

5. Guliani B, Agarwal I, Naik M. Effect of uncomplicated cataract surgery on central macular thickness in diabetic and Non-diabetc subjects. J Ophthalmic Vis Res 2019;14:442-7.

6. Sahin M, Cingu A, Gozum N. Evaluation of cystoid macular edema using optical coherence tomography and fundus autofluorescence after uncomplicated phacoemulsification surgery. J Ophthalmol 2013;376013. 
7. Katsimpris J, Petropoulos I, Zoukas G, Patokos T, Brinkmann C, Theoulakis $P$. Central fovea thickness before and after cataract surgery in normal and in diabetic patients with retinopathy. Klinische Monatsblatter fur Augenheilkunde. 2012;229:331-37.

8. Chu C, Johnston R, Buscombe C, Sallam A, Mohamed Q, Yang Y, et al. Risk Factors and incidence of macular edema after cataract surgery: A database study of 81984 eyes. Ophthalmology 2016:123:316-23.

9. Yang J, Cai L, Sun Z, Ye H, Fan Q, Zhang K, et al. Risk factors for and diagnosis of pseudophakic cystoid macular edema after cataract surgery in diabetic patients. J Cataract Refractive Surg. 2017;43:207-14.

10. Dowler J, Sehmi K, Hykim P, Hamilton A. The natural history of macular edema after cataract surgery in diabetes. Ophthalmol. 1999;106:663-68.

11. Diabetic Retinopathy Clinical Research Network. An Observational Study in Individuals with Diabetic Retinopathy without Center-Involved DME Undergoing Cataract Surgery. Protocol Q, version 1.0, July 2009.

12. Terada $Y$, Masuda A, Nejima R, Maruyama $Y$, et al. The anti-inflammatory effect of $0.1 \%$ bromfenac combination in post-cataract surgery patients with diabetes mellitus. Nippon Ganka Gakkai zasshi. 2014;118:645-51.

13. Singh R, Lehmann R, Martel J, Jong K, Pollack A, Tsorbatzoglou A, et al. Nepafenac $0.3 \%$ after cataract surgery in patients with diabetic retinopathy. Ophthalmol. 2017;124:776-85.

14. Grzybowski A, Kanclerz P, Huerva V, Ascaso F, Tuuminen J. Diabetes and Phacoemulsification Cataract Surgery: Difficulties, Risks and Potential Complications. J Clin Med 2019;8:716.

15. Early treatment diabetic retinopathy study research group. Photocoagulation for diabetic macular edema. Early treatment diabetic retinopathy study report number 1. Arch Ophthalmol. 1985;103:1796-1806.

16. Moshfeghi A, Thompson D, Berliner A, Sarg N. Outcomes in Patients with Diabetic Macular Edema Requiring Cataract Surgery in VISTA and VIVID Studies. Ophthalmol Retina 2020;4:481-5.
17. Boyer DS, Yoon $\mathrm{YH}$, Belfort R Jr, et al. Three-year, randomized, sham-controlled trial of dexamethasone intravitreal implant in patients with diabetic macular edema. Ophthalmology. 2014;121:1904-1914.

18. Takamura Y, Kubo E, Akagi Y. Analysis of the effect of intravitreal bevacizumab injection on diabetic macular edema after cataract surgery. Ophthalmology. 2009;116:1151-1157.

19. Lanzagorta A, Palacios E, Menezo J, Navea A. Prevention of vision loss after cataract surgery in diabetic macular edema with intravitreal bevacizumab: a pilot study. Retina. 2009;29:530-35.

20. Lam D, Chan C, Mohamed S, Lai T, Lee V, Lai W, et al. Phacoemulsification with intravitreal triamcinolone in patients with cataract and coexisting diabetic macular oedema: a 6-month prospective pilot study. Eye. 2005;19:885-90.

21. Takata C, Messias A, Folgosa M, Lucena L, Lucena D, Scott I, et al. Intravitreal injection versus subtenon infusion of triamcinolone acetonide during cataract surgery in patients with refractory diabetic macular edema. Retina. 2010;30:562-69.

22. Lim L, Morrison J, Constantinou M et al. Diabetic macular edema at the time of cataract surgery trial: a prospective, randomized clinical trial of intravitreous bevacizumab versus triamcinolone in patients with diabetic macular oedema at the time of cataract surgery - preliminary 6-month results. Clin Exp Ophthalmol. 2016;44:233-242.

23. Sze A, Luk F, Yip T, Lee G, Chan C. Use of intravitreal dexamethasone implant in patients with cataract and macular edema undergoing phacoemulsification. Eur J Ophthalmol. 2014; https://doi.org/10.5301/ ejo.5000523.

24. Agarwal A, Gupta V, Ram J, Gopta A. Dexamethasone intravitreal implant during phacoemulsification. Ophthalmol. 2013;120:211-17.

25. Panozzo G, Gusson E, Panozzo G, Dalla G. Dexamethasone intravitreal implant at the time of cataract surgery in eyes with diabetic macular edema. Eur J Ophathalmol. 2016; https://doi.org/10.5301/ejo.5000920. 


\title{
Indicaciones de la vitrectomía en el edema macular diabético
}

\author{
Sergio Hernández-Da Mota* \\ Servicio de Retina, Clínica David, Morelia, Michoacán, México
}

Los pacientes con retinopatía diabética, en caso de no tratarse, pueden sufrir una pérdida visual grave. En los países desarrollados, la retinopatía diabética constituye la principal causa de ceguera en la población en edad laboral. Desde la introducción de la fotocoagulación panretiniana en la práctica clínica, la tasa de pérdida visual se ha reducido enormemente en los países desarrollados. Por otro lado, el edema macular diabético (EMD) sigue siendo la causa más común de pérdida visual moderada en los pacientes diabéticos ${ }^{1,2}$.

\section{Tratamiento actual del edema macular diabético}

Varios ensayos clínicos aleatorizados han demostrado que los agentes que bloquean al factor de crecimiento endotelial vascular (VEGF, vascular endothelial growth factor) superan a la fotocoagulación con láser macular en el tratamiento del EMD con involucro central. Independientemente del agente anti-VEGF que se elija, hasta el $40 \%$ de los ojos tratados pueden tener una ganancia de $\geq 3$ líneas de agudeza visual mejor corregida (AVMC). Estos resultados han cambiado el algoritmo de tratamiento del EMD y actualmente los anti-VEGF son los fármacos de primera línea para el EMD con involucro central. Para lograr el máximo beneficio de la terapia anti-VEGF, la mayoría de los ojos requieren múltiples inyecciones por largos periodos de tiempo. Sin embargo, muchos pacientes en la vida real no reciben el tratamiento recomendado, por diferentes razones, y no alcanzan el potencial completo de la terapia anti-VEGF ${ }^{3,4}$.
Por otro lado, a pesar de este tratamiento intensivo, hasta el $60 \%$ de los ojos tendrán EMD persistente después de seis inyecciones mensuales consecutivas de un anti-VEGF. Además, las inyecciones intravítreas, aunque relativamente seguras, no están exentas de riesgos. Se calcula que el riesgo acumulado de endoftalmitis después de 20-40 inyecciones es de alrededor del 1\%. Como parte de las consideraciones económicas, los tratamientos farmacológicos son caros y representan un gasto y una carga importante. Las múltiples visitas y las inyecciones continuadas imponen una carga a los cuidadores, los sistemas de salud y los propios pacientes ${ }^{5-7}$.

Por estas razones, en muchas partes del mundo, la terapia crónica con anti-VEGF para el EMD es insostenible a largo plazo. Se necesita otra opción rentable y con un uso de recursos menos intensivo. La vitrectomía vía pars plana (VPP) se ha explorado en este sentido como una alternativa a la terapia anti-VEGF.

\section{El vítreo y la fisiopatología del edema macular diabético}

La patogenia del EMD es compleja y multifactorial, e incluye fuerzas de tracción anteroposterior y tangencial en la mácula, las cuales pueden causar o exacerbar el EMD. La interfase vitreomacular (IVM) está alterada en los pacientes diabéticos debido en parte a que la hiperglucemia crónica provoca la glicación no enzimática de las fibras de colágeno vítreo. Otro hallazgo en el EMD es la rotura de la barrera hematorretiniana, que permite que las citocinas se concentren en la interfase vitreorretiniana ${ }^{8,9}$. 
Se ha descrito que el desprendimiento vítreo posterior (DVP) ocurre en el $20 \%$ de los pacientes con EMD, en comparación con el $55 \%$ de aquellos sin EMD, lo que sugiere que la hialoides posterior podría estar ejerciendo tracción sobre la mácula y contribuir a la génesis del EMD. Las citocinas pueden estimular la migración de elementos celulares con propiedades contráctiles hacia la hialoides posterior engrosada, lo que induce la desestabilización de la matriz de colágeno y lleva a la tracción tangencial y al edema macular ${ }^{10,11}$.

Además de la hialoides posterior, las membranas epirretinianas (MER) y la membrana limitante interna (MLI) también pueden causar tracción sobre la retina. El examen histológico de la MLI en pacientes diabéticos ha demostrado que es mucho más gruesa que en los no diabéticos. Algunos han propuesto que, además de su efecto mecánico de tracción sobre la mácula, la MLI sirve como una barrera a la difusión fisiológica del agua y las citocinas, lo que contribuye al desarrollo de $\mathrm{EMD}^{12,13}$.

Por otro lado, las anormalidades en la IVM pueden estar presentes hasta en el $75 \%$ de los ojos con EMD. Se ha reportado que la tracción vitreomacular ocurre en un $4-25 \%$ de los ojos con EMD difuso. Hasta el $22.4 \%$ de los ojos pueden mostrar una MER ${ }^{14}$.

Existe controversia sobre si la presencia de anomalías de la IVM disminuye o no la eficacia de los fármacos intravítreos en los ojos con EMD. Algunos investigadores han señalado que la presencia de anomalías de la IVM no afecta negativamente los resultados de los agentes anti-VEGF intravítreos. En contraste, otros investigadores han informado una disminución de la eficacia de los anti-VEGF en ojos con EMD y MER concomitante ${ }^{15,16}$.

La VPP evita la tracción al eliminar la hialoides posterior, las MER y la MLI. Además, también puede contribuir a una disminución del EMD por medios no mecánicos.

La hipoxia es una de las principales causas de la secreción de VEGF, por lo que el alivio de esta causa una disminución en las concentraciones del VEGF. Debido a que la VPP aumenta la concentración de oxígeno en la cavidad vítrea, también puede disminuir la secreción de VEGF. Normalmente, el oxígeno se difunde desde las arterias retinianas hacia el vítreo avascular adyacente. La estructura gelatinosa del vítreo impide una difusión generalizada de oxígeno. Esta difusión está inversamente relacionada con la viscosidad del medio. El vítreo es mucho más viscoso que la solución salina, y antes de que el oxígeno tenga la oportunidad de difundirse, el ascorbato lo consume. La eliminación del gel vítreo elimina el ascorbato y disminuye la viscosidad del medio, lo que permite la distribución uniforme de oxígeno en el líquido de la cavidad vítrea. Una mayor concentración de oxígeno también puede conducir a vasoconstricción retiniana, que a su vez disminuye el grosor macular. Otro mecanismo mediante el cual la VPP puede reducir el EMD involucra al flujo sanguíneo retiniano ${ }^{17-20}$.

Es bien sabido que la hiperglucemia crónica altera la autorregulación del flujo sanguíneo retiniano. En ojos diabéticos con EMD clínicamente significativo se ha demostrado que la velocidad del flujo sanguíneo perifoveal se reduce en comparación con ojos diabéticos sin EMD, y se reduce aún más en ojos no diabéticos. Finalmente, la VPP elimina citocinas de la cavidad vítrea y permite, a su vez, una eliminación más rápida de citocinas recién secretadas, como el VEGF. La vida media del VEGF vítreo es diez veces más corta en ojos vitrectomizados que en aquellos que no lo están ${ }^{21-23}$.

\section{Condiciones en que se ha realizado vitrectomía pars plana para el edema macular diabético}

\section{Presencia de hialoides posterior engrosada y tensa}

La DRCR.net realizó un estudio observacional prospectivo de 87 ojos con EMD y tracción vitreomacular que se sometieron a VPP, la mayoría después de que fracasaran terapias previas para el EMD. Aunque casi todos los ojos tuvieron una mejoría en el grosor macular, no ocurrió así en la visión. Solo el 38\% de los ojos tuvieron una ganancia $\geq 10$ letras, y el $22 \%$ perdió $\geq$ 10 letras. Sin embargo, el estudio presenta varias limitaciones, entre ellas que la isquemia macular no se evaluó, por lo que posiblemente esta cohorte de pacientes puede haber tenido un potencial limitado de mejoría visual ${ }^{24}$.

\section{Adhesión vitreomacular sin presencia de engrosamiento o tracción maculares}

Se ha informado que la VPP es eficaz para resolver el EMD con una mejoría concomitante en la agudeza visual, incluso en ausencia de una hialoides posterior tensa y engrosada. Se ha descrito que una hialoides adherida podría ejercer tracción y contribuir al desarroIlo del EMD. Sin embargo, algunos estudios contradicen estos hallazgos ${ }^{25,26}$. 


\section{Hialoides posterior desprendida y presencia de membrana epirretiniana}

No está del todo claro de qué manera la MER contribuye a la formación del EMD. En este rubro, existe también evidencia contradictoria. En una pequeña serie de 30 ojos, la agudeza visual y el grosor de la retina mejoraron independientemente de la presencia o no de MER y de la presencia o no de DVP, lo que sugiere que la MER desempeña un papel modesto en la patogenia del EMD. Por el contrario, la DRCR.net informó que uno de los dos factores asociados con mejoría en la agudeza visual después del VPP fue la extracción de la MER ${ }^{26}$.

\section{Hialoides posterior desprendida sin anormalidades de la interfase vitreomacular}

En ojos sin ningún elemento de tracción también se ha descrito que la VPP puede llegar a ser benéfica. No obstante, igual que en la adhesión vitreomacular sin presencia de engrosamiento o tracción, así como en casos con hialoides posterior desprendida y MER, la evidencia es contradictoria ${ }^{27}$.

Algunos autores han propuesto que, en los ojos sin tracción vitreomacular obvia, la MLI puede ejercer tracción macular. Además, la MLI puede servir como un andamiaje para la formación de MER.

Se han publicado pocos ensayos clínicos aleatorizados de VPP para el tratamiento del EMD. Todos tienen el inconveniente de contar con un número pequeño de pacientes y un seguimiento relativamente corto, y se llevaron a cabo en la era de la fotocoagulación con láser macular. La mayoría de estos estudios reclutaron pacientes que tenían EMD persistente o recurrente a pesar de la fotocoagulación con láser macular previa. Hay también varias revisiones sistemáticas y metaanálisis publicados. De acuerdo con estas revisiones, la VPP no ofreció ningún beneficio visual en comparación con la fotocoagulación con láser macular o la observación, a pesar de la mejoría en el grosor retiniano (figura 1) 28-31.

Por otra parte, no está claro si la remoción de la MLI confiere un beneficio visual sobre la VPP sin remoción quirúrgica de esta; incluso se ha planteado un posible daño ultraestructural a las células de Müller en los ojos en que se elimina la MLI (figura 2).

Michalewska, et al. ${ }^{32}$ realizaron VPP en 44 pacientes consecutivos con EMD sin tratamiento previo. En su estudio, más de la mitad de los pacientes experimentaron una mejoría $\geq 3$ líneas de AVMC y solo el $2 \%$ perdieron $\geq 3$ líneas. Identificaron la presencia de MER preoperatoria, la duración de la diabetes mellitus y la agudeza visual inicial deficiente como factores asociados con una mala agudeza visual posquirúrgica. La recurrencia de EMD solo se observó en el 7\% de los ojos. Estos resultados son alentadores y sugieren que una intervención temprana con VPP puede ser benéfica, pero deben confirmarse en ensayos controlados prospectivos más grandes. En la actualidad es una práctica común considerar la VPP cuando todo lo demás ha fallado 26,32 .

Los factores pronósticos asociados con una mayor ganancia visual después de la VPP son no haberse sometido a fotocoagulación con láser macular previa, tener la hemoglobina glucosilada baja, una peor agudeza visual basal y remoción de la MER durante la cirugía. Los factores asociados con una mayor disminución del grosor macular incluyen la remoción de la MLI, la presencia de anormalidades en la IVM, una peor agudeza visual basal y un mayor grosor macular basal ${ }^{25,26}$.

La tomografía de coherencia óptica puede permitir una mejor estratificación de los ojos sometidos a VPP para el tratamiento del EMD. Se ha visto que en los ojos con el subtipo quístico de EMD no ha ido bien la VPP. También se ha advertido que el peeling de la MLI en ojos con quistes intrarretinianos grandes y una zona avascular foveal agrandada existe un riesgo significativo de causar atrofia subfoveal y pérdida visual. Por otro lado, el daño preoperatorio de la membrana limitante externa ha sido el predictor más fuerte para la mejoría visual. Por el contrario, el grosor macular central basal fue un predictor de mal pronóstico. Otros han identificado la presencia de líquido subretiniano como un biomarcador favorable ${ }^{33-36}$.

Algunos investigadores están en contra de la VPP primaria para el EMD porque creen que la farmacocinética intravítrea se altera después de la vitrectomía y, debido a que algunos ojos tienen EMD persistente o recurrente después de VPP, esto podría representar un problema. Sin embargo, el armamento actual contra el EMD incluye inyecciones de triamcinolona retrobulbares, implante de acetónido de fluocinolona e implante de dexametasona, los cuales han demostrado ser también eficaces en ojos vitrectomizados. Las complicaciones reportadas en la era de la VPP 20Ga incluyeron desgarros de retina, desprendimientos de retina regmatógenos, glaucoma neovascular, hemorragia vítrea recurrente, agujero macular lamelar, agujero macular de espesor total y 


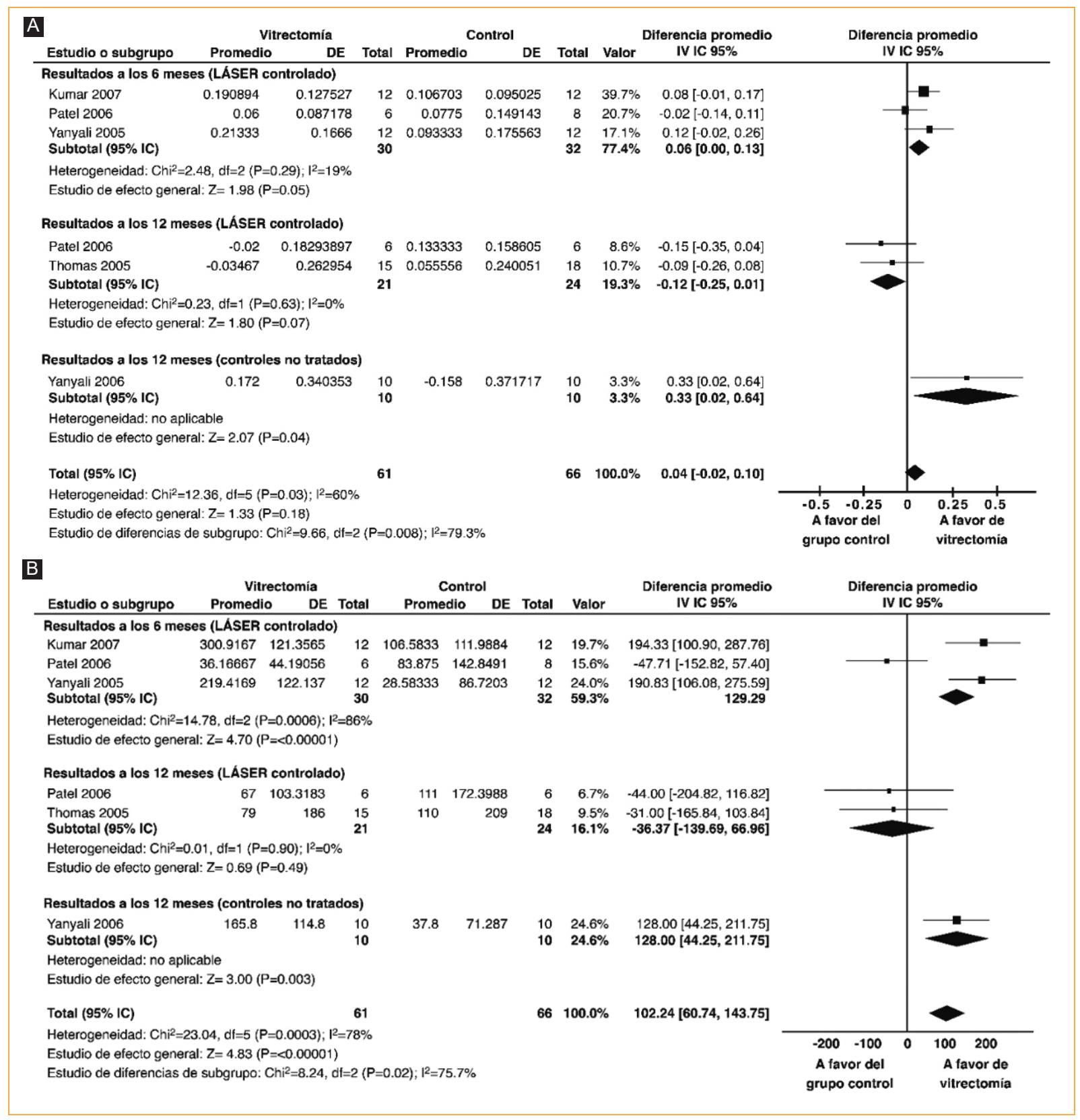

Figura 1. Metaanálisis que presenta los resultados visuales y de grosor macular comparando vitrectomía vía pars plana (VPP) con laserterapia u observación (grupo control). A: en el primer gráfico no hay diferencia significativa entre la VPP y los controles. B: en el segundo gráfico, los resultados favorecen a la VPP.

formación de MER. En las últimas dos décadas, las mejoras en las técnicas quirúrgicas y en la instrumentación han hecho que la VPP sea mucho más segura. El peeling de la MLI se ha facilitado con la cromovitrectomía ${ }^{37-40}$.

De acuerdo con lo anteriormente comentado, es posible concluir que los pacientes con EMD y evidente componente traccional se benefician del tratamiento quirúrgico. En casos refractarios al tratamiento convencional se podría reservar como tratamiento adyuvante a criterio del clínico. Para pacientes con EMD y engrosamiento de la MLI o sin componente traccional, el nivel de evidencia es bajo o contradictorio. Se requieren estudios con mayor nivel de evidencia para determinar el verdadero papel de la vitrectomía en el edema macular diabético. 


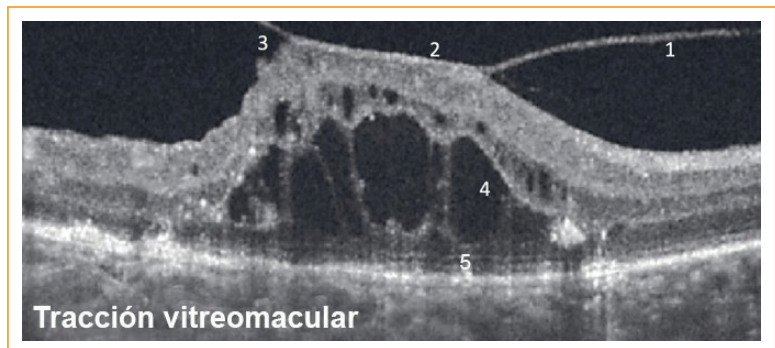

Figura 2. Tomografía de coherencia óptica de edema macular diabético con componente traccional.

1: evidencia de separación de la corteza vítrea de la superficie retiniana. 2: adherencia de la corteza vítrea dentro de un radio de $3 \mathrm{~mm}$ de la fóvea. 3: asociación de la adherencia con distorsión de la superficie foveal. 4: cambios estructurales intrarretinianos. 5: elevaciónseparación de la fóvea del epitelio pigmentario de la retina.

\section{Bibliografía}

1. Williams R, Airey M, Baxter H, Forrester J, Kennedy T, Girach A. Epidemiology of diabetic retinopathy and macular oedema: a systematic review. Eye (Lond). 2004;18:963-83.

2. Klein R, Klein B, Moss S, Cruickshanks K. The Wisconsin Epidemiologic Study of Diabetic Retinopathy XV: the long-term incidence of macular edema. Ophthalmology. 1995;102:7-16.

3. Brown D, Nguyen Q, Marcus D, Boyer D, Patel S, Feiner L, et al. Longterm outcomes of ranibizumab therapy for diabetic macular edema: the 36-month results from two phase-III trials: RISE and RIDE. Ophthalmology. 2013;120:2013-22.

4. Elman M, Ayala A, Bressler N, Browning D, Flaxel C, Glassman A, et al. Intravitreal ranibizumab for diabetic macular edema with prompt vs. referred laser treatment: 5-year randomized trial results. Ophthalmology. 2015;122:375-81.

5. Bressler N, Beaulieu W, Glassman A, Blinder K, Bressler S, Jampol L, et al. Persistent macular thickening following intravitreous aflibercept, bevacizumab or ranibizumab for central-involved diabetic macular edema with vision impairment: a secondary analysis of a randomized clinical trial. JAMA Ophthalmol. 2018;136:257-69.

6. McCannel C. Meta-analysis of endophthalmitis after intravitreal injection of anti-vascular endothelial growth factor agents: causative organisms and possible prevention strategies. Retina. 2011;31:654-61.

7. Smiddy W. Economic considerations of macular edema therapies. Ophthalmology. 2011:118:1827-33.

8. Kaiser P, Riemann C, Sears J, Lewis H. Macular traction detachment and diabetic macular edema associated with posterior hyaloidal traction. Am J Ophthalmol. 2001:131:44-9.

9. Lewis $\mathrm{H}$. The role of vitrectomy in the treatment of diabetic macular edema. Am J Ophthalmol. 2001;131:123-5.

10. Nasrallah F, Jalkh A, Van Coppenolle F, Kado M, Trempe C, McMeel J, et al. The role of the vitreous in diabetic macular edema. Ophthalmology. 1988;95:1335-9.

11. Harbour J, Smiddy W, Flynn H, Rubsamen P. Vitrectomy for diabetic macular edema associated with a thickened and taut posterior hyaloid membrane. Am J Ophthalmol. 1996;121:405-13.

12. Tamura K, Yokoyama T, Ebihara N, Murakami A. Histopathologic analysis of the internal limiting membrane surgically peeled from eyes with diffuse diabetic macular edema. Jpn J Ophthalmol. 2012;56:280-7.

13. Saravia M. Persistent diffuse diabetic macular edema. The role of the internal limiting membrane as a selective membrane: the oncotic theory. Med Hypotheses. 2011;76:858-60.

14. Ophir A, Martinez M, Mosqueda P, Trevino A. Vitreous traction and epiretinal membranes in diabetic macular oedema using spectral-domain optical coherence tomography. Eye (Lond). 2010;24:1545-53.

15. Chang $C$, Cheng $C$, Peng $C$. The incidence and risk factors for the development of vitreomacular interface abnormality in diabetic macular edema treated with intravitreal injection of anti-VEGF. Eye (Lond). 2017;31:762-70.
16. Yoon D, Rusu I, Barbazetto I. Reduced effect of anti-vascular endothelial growth factor agents on diabetics with vitreomacular interface abnormalities. Int Ophthalmol. 2014;34:817-23.

17. Vinores S, Youssri A, Luna J, Chen $Y$, Bhargave S, Vinores $M$, et al. Upregulation of vascular endothelial growth factor in ischemic and non-ischemic human and experimental retinal disease. Histol Histopathol. 1997;12:99-109.

18. Barbazetto I, Liang J, Chang S, Zheng L, Spector A, Dillon J. Oxygen tension in the rabbit lens and vitreous before and after vitrectomy. Exp Eye Res. 2004;78:917-24.

19. Gisladottir S, Loftsson T, Stefansson E. Diffusion characteristics of vitreous humour and saline solution follow the stokes Einstein equation. Graef Arch Clin Exp Ophthalmol. 2009;247:1677-84.

20. Stefánsson E. Physiology of vitreous surgery. Graef Arch Clin Exp Ophthalmol. 2009:247:147-63.

21. Ciulla $T$, Harris A, Latkany P, Piper $H$, Arend $O$, Garzozi $H$, et al. Ocular perfusion abnormalities in diabetes. Acta Ophthalmol Scand. 2002;80:468-77.

22. Sakata K, Funatsu H, Harino S, Noma H, Hori S. Relationship between macular microcirculation and progression of diabetic macular edema. Ophthalmology. 2006;113:1385-91.

23. Lee S, Ghosn C, Yu Z, Zacharias L, Kao H, Lanni C, et al. Vitreous VEGF clearance is increased after vitrectomy. Invest Ophthalmol Vis Sci. 2010;51:2135-8.

24. Haller J, Qin H, Apte R, Beck R, Bressler N, Browning D, et al. Vitrectomy outcomes in eyes with diabetic macular edema and vitreomacular traction. Ophthalmology. 2010;117:1087-93.

25. La Heij E, Hendrikse F, Kessels A, Derhaag P. Vitrectomy results in diabetic macular oedema without evident vitreomacular traction. Graef Arch Clin Exp Ophthalmol. 2001;239:264-70.

26. Flaxel C, Edwards A, Aiello L, Arrigg P, Beck R, Bressler N, et al. Factors associated with visual acuity outcomes after vitrectomy for diabetic macular edema: diabetic retinopathy clinical research network. Retina. 2010;30:1488-95.

27. Ikeda T, Sato K, Katano T, Hayashi Y. Improved visual acuity following pars plana vitrectomy for diabetic cystoid macular edema and detached posterior hyaloid. Retina. 2000;20:220-2.

28. Messmer E, Heidenkummer H, Kampik A. Ultrastructure of epiretinal membranes associated with macular holes. Graef Arch Clin Exp Ophthalmol. 1998:236:248-54

29. Kampik A, Kenyon K, Michels R, Green W, de la Cruz Z. Epiretinal and vitreous membranes. Comparative study of 56 cases. Arch Ophthalmol. 1981:99:1445-54.

30. Jackson T, Nicod E, Angelis A, Grimaccia F, Pringle E, Kanavos P. Pars plana vitrectomy for diabetic macular edema: a systematic review, meta-analysis, and synthesis of safety literature. Retina. 2017;37:886-95.

31. Simunovic M, Hunyor A, Ho I. Vitrectomy for diabetic macular edema: a systematic review and meta-analysis. Can J Ophthalmol. 2014;49:188-95.

32. Michalewska Z, Stewart M, Landers M, Bednarski M, Adelman R, Nawrocki J, et al. Vitrectomy in the management of diabetic macular edema in treatment-naïve patients. Can J Ophthalmol. 2018:53:402-7.

33. Avci R, Kaderli B, Avci B, Simsek S, Baykara M, Kahveci Z, et al. Pars plana vitrectomy and removal of the internal limiting membrane in the treatment of chronic macular oedema. Graef Arch Clin Exp Ophthalmol. 2004;242:845-52

34. Romano M, Romano V, Vallejo J, Vinciguerra R, Romano M, Cereda M, et al. Macular hypotrophy after internal limiting membrane removal for diabetic macular edema. Retina. 2014;34:1182-9.

35. Iglicki M, Lavaque A, Ozimek M, Negri H, Okada M, Chhablani J, et al. Biomarkers and predictors for functional and anatomic outcomes for small gauge pars plana vitrectomy and peeling of the internal limiting membrane in naïve diabetic macular edema: the VITAL study. PLoS One. 2018;13:e200365

36. Ichiyama $Y$, Sawada O, Mori T, Fujikawa M, Kawamura H, Ohji M. The effectiveness of vitrectomy for diffuse diabetic macular edema may depend on its preoperative optical coherence tomography pattern. Graef Arch Clin Exp Ophthalmol. 2016;254:1545-51.

37. Koga T, Mawatari $Y$, Inumaru J, Fukushima M, Tanihara H. Trans-tenon's retrobulbar triamcinolone acetonide infusion for refractory diabetic macular edema after vitrectomy. Graef Arch Clin Exp Ophthalmol. 2005;243:1247-52.

38. Meireles A, Goldsmith C, El-Ghrably I, Erginay A, Habib M, Pessoa B, et al. Efficacy of $0.2 \mu \mathrm{g} /$ day fluocinolone acetonide implant (ILUVIEN) in eyes with diabetic macular edema and prior vitrectomy. Eye (Lond). 2017:31:684-90.

39. Boyer D, Faber D, Gupta S, Patel S, Tabandeh H, Li X, et al. Dexamethasone intravitreal implant for treatment of diabetic macular edema in vitrectomized patients. Retina. 2011:31:915-23

40. Pessoa B, Dias D, Baptista P, Coelho C, Beirão J, Meireles A. Vitrectomy outcomes in eyes with tractional diabetic macular edema. Ophthalmic Res. 2019;61:94-9. 


\section{Importancia del control sistémico de la diabetes mellitus tipo 2 en la retinopatía diabética y el edema macular diabético (importancia de la presión arterial, los lípidos, la hemoglobina glucosillada y la nefropatía)}

\section{Fabiola Hernández-Núñez*}

Hospital de Especialidades Bernardo Sepúlveda, Centro Médico Nacional Siglo XXI, Instituto Mexicano del Seguro Social, Ciudad de México, México

La retinopatía diabética $(\mathrm{RD})$ es una de las principales causas de baja visual en adultos en edad laboral en todo el mundo. El edema macular diabético (EMD) es una causa principal de discapacidad de la visión central en pacientes con RD. Diversos estudios clínicos sobre la epidemiología de la diabetes y de sus complicaciones, realizados desde hace décadas, han evidenciado que el control sistémico adecuado de la enfermedad y de varios factores de riesgo asociados, como la hiperglucemia, la hipertensión arterial (HTA), la dislipidemia y otros, disminuye el riesgo de progresión de la RD, del EMD y de la pérdida visual secundaria $^{1}$ (nivel de evidencia 1):

- DCCT: estudio del control de la diabetes y de la epidemiología de las complicaciones y de las intervenciones en diabetes (1983-1993), que demuestra que un control estricto de la glucemia en los pacientes con diabetes tipo 1, manteniendo un valor medio de la $(\mathrm{HbA} 1 \mathrm{c})$ del $7.2 \%$, disminuye el riesgo de progresión de retinopatía en un $76 \%$, reduce un $47 \%$ el desarrollo de retinopatía grave o proliferativa, un 56\% la necesidad de láser y un $23 \%$ el riesgo de EMD, y establece una relación lineal entre las cifras de $\mathrm{HbA} 1 \mathrm{c}$ y el riesgo de complicaciones visuales, pero advierte que los pacientes con control estricto tienen mayor número de eventos de hipoglucemia, que pueden ser peligrosos ${ }^{2}$.
- UKPDS: estudio prospectivo de diabetes tipo 2 (19771999) que constató hallazgos similares y demostró la necesidad de controlar la diabetes, así como el riesgo del colesterol elevado y de concentraciones de lípidos séricos, que aumentan el riesgo de complicaciones retinianas en los diabéticos (nivel de evidencia 1, grado de recomendación A). Además, demostró que el control estricto de la presión arterial disminuye un $34 \%$ la progresión de la RD y un $47 \%$ el deterioro de la agudeza visual (nivel de evidencia 1$)^{3}$.

- FIELD: estudio controlado aleatorizado (2007) sobre el efecto del fenofibrato en la necesidad de tratamiento láser para la RD que constató que el uso de $200 \mathrm{mg} /$ día de fenofibrato reduce la frecuencia de láser por EMD en un $31 \%$ y por RD proliferativa (RDP) en un $30 \%$, en pacientes con RD preexistente (este efecto benéfico no se observó en pacientes sin $\mathrm{RD}$ ) (nivel de evidencia 1) ${ }^{4}$.

- ACCORD: estudio de los efectos de las terapias médicas sobre la progresión de la RD en la diabetes tipo 2 (2010), que encontró que el tratamiento con fenofibrato se asoció a una disminución del $40 \%$ en la progresión de la RD (definida como 3 puntos o más en la escala del ETDRS [Early Treatment Diabetic Retinopathy Study] o la aparición de una RDP que precisara FPR o vitrectomía) (nivel de evidencia 1$)^{5,6}$. 
- WESDR: estudio epidemiológico de Wisconsin de la retinopatía diabética sobre la incidencia en 25 años de EMD en personas con diabetes tipo 1 (2008), que reportó que los valores de HbA1c son un factor predictivo de desarrollo de EMD en pacientes con un seguimiento de 25 años ${ }^{7}$.

A partir de estos y otros estudios clínicos aleatorizados de fase III se han emitido diversas recomendaciones internacionales y regionales, con la finalidad de obtener las mejores posibilidades de éxito en los tratamientos de la RD y del EMD. Las recomendaciones son orientativas, ya que se debe individualizar según las situaciones especiales de cada paciente.

En general, se recomienda que el control sistémico adecuado del paciente con diabetes se realice desde la etapa de diagnóstico, de forma coordinada con el médico de primer contacto, antes de que se desarrolle la $\mathrm{RD}$, y debe mantenerse durante toda la vida, siendo más estrictos en los objetivos durante los primeros años después del diagnóstico. Una vez establecido el diagnóstico de RD, es fundamental informar y concientizar al paciente sobre la importancia del control metabólico (nivel de evidencia 1, grado de recomendación A).

La participación multidisciplinaria con las especialidades de medicina interna, endocrinología, cardiología y nefrología, en conjunto con el oftalmólogo, brindará una mayor posibilidad de lograr los objetivos del tratamiento. Existe información relevante de los protocolos de tratamiento, tanto sistémico como oftalmológico, que todo el grupo de médicos tratantes tiene que conocer. La enfermedad ocular retiniana no debe tratarse como una afección aislada. En todos los estadios de la $\mathrm{RD}$ es esencial el control endocrinológico, fundamentalmente de la glucemia (HbA1c), la dislipidemia, la HTA, el sobrepeso y el estado renal (microalbuminuria en orina), además de otros factores como el estado nutricional y psicosocial del paciente. También debe recomendarse al paciente dejar de fumar (nivel de evidencia 1 , grado de recomendación A).

El tratamiento intensivo de la glucosa es más eficaz cuando se introduce como prevención primaria de la $\mathrm{RD}$ durante los primeros 5 años de la diabetes que cuando se hace como intervención secundaria después de que las complicaciones han comenzado a desarrollarse.

El control intensivo de la glucemia en los pacientes con diabetes disminuye la progresión de la $\mathrm{RD}$ y la aparición del EMD (ACCORD), además de conferir un efecto de «memoria metabólica» que persiste durante los siguientes años. Se sugiere determinar la $\mathrm{HbA1c}$ al menos dos veces al año en los pacientes diabéticos con control glucémico estable, y al menos cuatro veces al año en aquellos sin control glucémico estable ${ }^{5,6}$.

$\mathrm{Se}$ recomienda una $\mathrm{HbA} 1 \mathrm{C}<7 \%$ para el control glucémico en la mayoría de los pacientes, mientras que en pacientes seleccionados puede haber algunos beneficios al establecer un objetivo más bajo, del 6.5\%. De hecho, un aumento de la HbA1c supone un mayor riesgo de EMD (nivel de evidencia $1 A)^{8,9}$.

En algunos casos, una mejoría rápida del control de la glucemia y de la HbA1c puede provocar un empeoramiento de la RD y del EMD, que a veces producen una disminución de la visión, principalmente en los pacientes con un mayor descontrol metabólico y una etapa más avanzada de la RD. No obstante, a largo plazo, el control intensivo de la glucosa, comparado con el control convencional, se asocia a mejores resultados, por lo que la recomendación es optimizar el control glucémico para disminuir el riesgo de progresión de la RD y de aparición de EMD ${ }^{5,6}$. La monitorización oftalmológica antes del inicio del tratamiento intensivo y a intervalos de 3 meses durante 6 a 12 meses a partir de entonces parece apropiado para tales pacientes ${ }^{8,9}$.

En los pacientes cuya RD ya se está acercando a la etapa de alto riesgo puede ser prudente retrasar el inicio del tratamiento intensivo hasta que se pueda completar la fotocoagulación, en particular si la HbA1c es alta ${ }^{8,9}$.

También la HTA se asocia a un aumento del riesgo de progresión de la RD y aparición de EMD. Disminuir las cifras de presión arterial sistémica beneficia varios aspectos de la RD (disminuye su progresión y la necesidad de tratamiento con láser) (grado de recomendación $A)^{10,11}$.

Las cifras de presión arterial recomendadas son $\leq 140 / 80 \mathrm{mmHg}$ (nivel de evidencia 1, grado de recomendación $A)^{10,11}$.

Existen tratamientos que también pueden retrasar la progresión de la RD. Algunos fármacos que bloquean el sistema renina-angiotensina, como los inhibidores de la enzima convertidora de angiotensina (IECA) y los antagonistas de los receptores de la angiotensina II (ARA-II), han demostrado ser útiles para este fin. El enalapril (IECA) y el losartán (ARA-II) redujeron el riesgo de progresión de la $\mathrm{RD}$ en un $63 \%$ en pacientes con diabetes mellitus tipo 1, pero su papel en la exacerbación de la RD no ha recibido mucha atención. La angiotensina II es perjudicial en la RD al aumentar los principales eventos involucrados en su patogénesis, a saber, el estrés oxidativo, la angiogénesis y la inflamación. El uso de ARA-II ha mostrado resultados 
positivos en estudios de investigación y, por lo tanto, podrían proporcionar una nueva terapia adyuvante para tratar la RD y prevenir la pérdida visual asociada; sin embargo, aún no hay resultados concluyentes y los datos actuales deben tomarse con cautela ${ }^{10,11}$.

Respecto a los lípidos, los agentes hipolipidemiantes han demostrado tener un efecto positivo en la disminución de la progresión de la diabetes y de la $\mathrm{RD}$. Las cifras ideales de colesterol total deben ser < $200 \mathrm{mg} / \mathrm{dl}$, las de triglicéridos < $150 \mathrm{mg} / \mathrm{dl}$ y las de colesterol unido a lipoproteínas de alta densidad (C-HDL) $>40 \mathrm{mg} / \mathrm{dl}^{12}$.

En el ensayo ACCORD se vinculó la RD no proliferativa (RDNP) grave con un mayor número de eventos cardiovasculares, y se halló también una asociación positiva con el desarrollo de nefropatía, ${ }^{5,6}$.

A todos los pacientes diabéticos se les debe estimular para hacer un cambio hacia la vida saludable, reduciendo las grasas saturadas y trans, incrementando la ingesta de ácidos grasos poliinsaturados omega-3, fibra y estanoles/esteroles, y realizando actividad física para mejorar el perfil lipídico".

El objetivo de C-LDL en los pacientes con riesgo cardiovascular muy alto es $<70 \mathrm{mg} / \mathrm{dl}$, en aquellos con riesgo cardiovascular alto es $<100 \mathrm{mg} / \mathrm{dl}$ y en los de riesgo cardiovascular moderado o bajo es $<115 \mathrm{mg} / \mathrm{dl}$ (Piepoli, 2016). Del mismo modo, se debe optimizar el control glucémico si los triglicéridos están elevados $(\geq$ $150 \mathrm{mg} / \mathrm{dl}[1.7 \mathrm{mmol} / \mathrm{l}])$ o el C-HDL está bajo $(<40 \mathrm{mg} /$ dl $[1.0 \mathrm{mmol} / \mathrm{l}]$ en varones $\mathrm{y}<50 \mathrm{mg} / \mathrm{dl}[1.3 \mathrm{mmol} / \mathrm{l}]$ en mujeres) 1.

El uso de estatinas también se ha reportado benéfico en la RD. Los pacientes diabéticos reciben tratamiento con estatinas de acuerdo con su riesgo cardiovascular; en los que tienen antecedente de enfermedad cardiovascular, y de todas las edades, se debe añadir una estatina de alta intensidad a la modificación de los estilos de vida (grado de recomendación A). En los pacientes con diabetes mellitus tipo 2 de 40-75 años de edad (grado de recomendación A) y los mayores de 75 años sin enfermedad cardiovascular previa (grado de recomendación $\mathrm{B}$ ) se recomiendan estatinas de moderada intensidad ${ }^{1}$.

El fenofibrato ha mostrado su utilidad en retrasar la progresión de la RDNP leve y moderada hacia formas más graves, lo que le ha permitido que en algunos países se acepte su uso para esta indicación (nivel de evidencia 1 , grado de recomendación $A)^{4-6}$.

En cuanto a las precauciones con el uso de fenofibrato, hay que tener en cuenta la posibilidad de miopatía en combinación con una estatina (aunque esto no se reportó en los ensayos FIELD y ACCORD), que puede aumentar las transaminasas y la creatinina, y que se relaciona con un mayor riesgo de cálculos biliares. También se indica precaución cuando se utiliza fenofibrato en pacientes que reciben anticoagulantes cumarínicos orales. En el ensayo ACCORD se vinculó la RDNP grave con un mayor número de eventos cardiovasculares y se halló también una asociación positiva con el desarrollo de nefropatía ${ }^{4-6}$.

En prevención primaria se suele utilizar ácido acetilsalicílico (75-162 mg/d) en las personas con diabetes mellitus tipo 2 que tienen un alto riesgo cardiovascular. Con la evidencia actual (ARRIVE, ASPREE y ASCEND), el uso de ácido acetilsalicílico en general puede no recomendarse, pero no se contraindica en la RD y el EMD ${ }^{12}$.

\section{Efecto de la hemoglobina glucosilada, la presión arterial y los lípidos sobre el edema macular diabético}

Aunque la patogenia del EMD no se ha aclarado por completo, se sabe que es un cuadro de etiopatogenia multifactorial y compleja en cuyo desarrollo participan algunos factores de crecimiento, fundamentalmente el factor de crecimiento endotelial vascular (VEGF, vascular endothelial growth factor) y determinadas citocinas inflamatorias (interleucinas 6 y 8, proteína quimioatrayente de monocitos [MCP-1], etc.), y con una evolución que se ve modificada por la existencia de una serie de factores de riesgo, como son el grado de control metabólico, los valores de HbA1c, la duración de la diabetes, la HTA y la dislipidemia.

Si en la RD el control sistémico es importante, en el EMD resulta aún más relevante. Como ya se mencionó, existen varios estudios que señalan el mal control metabólico como un factor de riesgo de progresión del EMD. De acuerdo con estos datos, se recomienda también mantener la $\mathrm{HbA} 1 \mathrm{C}$ por debajo del $7 \%$; de hecho, un aumento de la $\mathrm{HbA1c}$ corresponde a un mayor riesgo de EMD (nivel de evidencia $1 A$ ). Sin embargo, en pacientes con diabetes mellitus tipo 2 de larga evolución y con comorbilidad, mantener la $\mathrm{HbA1c}$ en esos niveles conlleva el riesgo de hipoglucemias y de un aumento de la morbimortalidad (ACCORD y ADVANCE), por lo que los valores aconsejables de $\mathrm{HbA1C}$ se encuentran en torno al 7-8.5\% (nivel de evidencia 1 , grado de recomendación $A)^{5,6}$.

El Early Diabetic Retinopathy Study Group llegó a la conclusión de que unas cifras elevadas de lípidos séricos incrementan el riesgo de desarrollar exudados 
duros en la mácula. Sin embargo, cuando la HbA1c se controla, esta asociación no se confirma 4 .

Respecto al EMD y la HTA, el estudio WESDR consideró que la presión arterial diastólica basal es un factor de riesgo relativo para el desarrollo de EMD en pacientes con diabetes mellitus tipo 2, pero no en aquellos con diabetes mellitus tipo 1. Las cifras de presión arterial recomendadas son $\leq 140 / 80 \mathrm{mmHg}^{4}$.

El efecto del fenofibrato para mejorar el EMD parece ser mucho más modesto, sin que se aprecien mejoras con significación estadística 4 .

La atención a la respuesta psicológica del paciente a la RD y la baja visual por EMD es importante. Detectar los datos de ansiedad y depresión oportunamente ayudará a referir al paciente de forma más rápida para que reciba la atención necesaria.

\section{Efecto del control sistémico sobre el resultado del tratamiento del edema macular}

La fotocoagulación con láser, el estándar de atención del EMD durante décadas, prevenía principalmente la pérdida de visión, pero con frecuencia no mejoraba la agudeza visual. La inyección intravítrea de agentes anti-VEGF se ha convertido en el tratamiento de primera línea para los pacientes con EMD con involucro del subcampo central, y los esteroides como segunda línea (RISE, RIDE, RESTORE, RESOLVE, VIVID, VISTA, MEAD, IRGREL-DEX, etc.), pero a pesar de los beneficios comprobados de la terapia anti-VEGF y esteroidea, un subgrupo de pacientes responden mal a estos tratamientos y tienen EMD persistente incluso después un tratamiento intensivo. El desafío futuro a corto plazo es poder predecir la respuesta individual a las terapias actuales, asociadas a los factores sistémicos concomitantes que pueden facilitar los mejores resultados visuales ${ }^{1}$.

Mientras los grandes ensayos clínicos y estudios epidemiológicos han demostrado que un control estricto de la $\mathrm{HbA1c}$, la presión arterial y los lípidos puede retrasar significativamente la aparición y la progresión de la RD y el EMD, la influencia de estos mismos factores sobre los resultados visuales y anatómicos del tratamiento con anti-VEGF y esteroideo para el EMD no está clara. Los resultados de los diversos ensayos clínicos de fase III y sus análisis post hoc no han mostrado resultados concluyentes.

En los pacientes tratados con anti-VEGF se han asociado las cifras bajas de HbA1c con la disminución del grosor del subcampo foveal, pero no se ha confirmado una relación con la agudeza visual (Signht, et al. y Zaid, et al.).

En un estudio de pacientes tratados con ranibizumab (RISE y RIDE) no se encontró asociación entre el cambio medio en la agudeza visual mejor corregida y el valor basal de la HbA1c en las semanas 52 y 100 del estudio. Esto contrasta con los resultados en pacientes tratados con aflibercept (VISTA y VIVID), que señalan que la mejoría media en agudeza visual a los 2 años dependía de los valores de la HbA1c. Más recientemente, un análisis exploratorio (post hoc) del Protocolo T de DRCR.net también ha encontrado que la magnitud de la mejoría de la visión después del tratamiento anti-VEGF se asocia a la HbA1c, y que por cada aumento de un $1 \%$ sobre los valores basales de la HbA1c, el cambio de agudeza visual a más de 2 años fue de -0.5 letras (intervalo de confianza del $95 \%$ : -0.9 a -0.2$)^{13,14}$.

Hay varias explicaciones posibles a la discrepancia entre los resultados de estudios de pacientes con valores de $\mathrm{HbA} 1 \mathrm{c}$ similares, como que estos puedan tener marcadas diferencias en sus perfiles diarios de glucosa. Los picos hiperglucémicos transitorios pueden ser un factor de riesgo para complicaciones independientes de la HbA1c, debido a episodios transitorios de estrés oxidativo. La isquemia capilar macular subyacente puede explicar la no mejoría visual en los ojos crónicos y refractarios de EMD, independientemente de los valores de HbA1c. Campochiaro, et al. han propuesto un circuito de retroalimentación positiva para el VEGF, en el cual la oclusión vascular causa isquemia retiniana y las células de la retina isquémica liberan VEGF, lo que lleva a la exacerbación de la no perfusión retiniana por la promoción de leucostasis, un cambio asociado con la inflamación, y esto puede ser otra razón por la cual en la práctica cotidiana falla la respuesta tanto anatómica como funcional a los antiangiogénicos. Además, la mayoría de los estudios han utilizado la HbA1c medida en el momento de la inyección, lo que solo refleja el control de la glucosa en sangre en los 3 meses anteriores y no después de administrar el tratamiento. También existen pocos estudios que hayan incluido poblaciones con una $\mathrm{HbA1c}>$ $12 \%$. Por tanto, los ensayos clínicos no reflejan la población que se ve en la práctica médica real.

Respecto al efecto de la HTA en el tratamiento del edema macular, el UKPDS mostró que una reducción media de la presión arterial sistólica de $10 \mathrm{mmHg}$ y una reducción de la presión arterial diastólica de $5 \mathrm{mmHg}$ durante un seguimiento medio de 8.4 años condujo a 


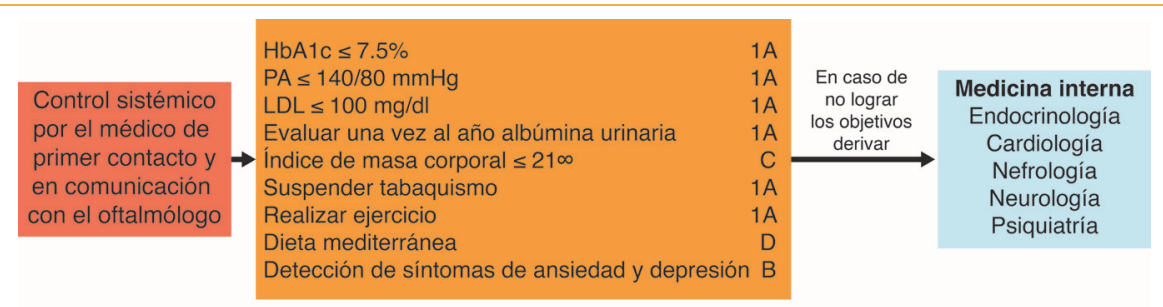

El control de todos los factores en conjunto puede reducir el riesgo de aparición y progresión de la RD Su influencia sobre el resultado del tratamiento del edema macular con anti-VEGF y esteroides no está clara Las cifras sugeridas son solo orientativas, cada paciente debe tratarse de forma individual

Figura 1. Niveles de evidencia y grados de recomendación para el control sistémico de la retinopatía diabética (RD) y el edema macular diabético (EMD). HbA1c: hemoglobina glucosilada; LDL: lipoproteínas de baja densidad;

PA: presión arterial.

una reducción del $35 \%$ en los tratamientos con láser de la retina, de los cuales el $78 \%$ fueron para $\mathrm{EMD}^{3}$.

El control de la HTA sigue siendo importante por su efecto sobre los resultados del tratamiento del EMD, aunque los beneficios de su manejo intensivo tampoco son concluyentes ${ }^{3}$.

Las tiazolidinedionas son agentes orales utilizados para tratar la diabetes mellitus tipo 2. Son agonistas de los receptores gamma activados por el proliferador de peroxisomas que funcionan mejorando la sensibilidad a la insulina. La pioglitazona y la rosiglitazona son miembros de esta clase de medicamentos de uso común. Los valores plasmáticos de VEGF son más altos en los pacientes que reciben tiazolidinedionas que en aquellos que no toman estos medicamentos. Los informes de casos y los estudios de cohorte de base de datos retrospectivos sugieren que también pueden asociarse con EMD nuevo o empeorado, pero en el estudio ACCORD el uso de tiazolidinedionas no se asoció con la prevalencia de EMD al inicio del estudio ni con la incidencia de EMD durante 4 años de seguimiento ${ }^{5,6}$.

En un pequeño estudio prospectivo (UKPDS), los pacientes con triglicéridos anormalmente elevados y C-HDL disminuido tuvieron peores resultados de agudeza visual después de la fotocoagulación focal/cuadrícula que aquellos con cifras normales ${ }^{3}$.

Los agentes hipolipidemiantes han demostrado tener un efecto positivo en la disminución de la progresión de la diabetes y de la RD. En un metaanálisis, los agentes hipolipidemiantes se asociaron a una posible reducción del riesgo de desarrollar EMD, pero a pesar de esto no tuvieron efecto sobre la agudeza visual ni sobre la presencia de exudados duros en la retina ${ }^{3}$.

Existe evidencia contradictoria en cuanto a que la genética y los factores epigenéticos puedan explicar las diferencias en las tasas de progresión de la RD entre grupos de individuos con una duración similar de la diabetes o con valores similares de HbA1c. Un estudio encontró que los haplogrupos genéticos mitocondriales modifican el riesgo de progresión de la enfermedad a pesar de presentar similares $\mathrm{HbA1c}$ y duración de la diabetes. Para los pacientes con el haplogrupo $\mathrm{H}$, la mayor duración de la diabetes y el aumento de la $\mathrm{HbA} 1 \mathrm{C}$ fueron factores de riesgo significativos para RDP. Sin embargo, en los pacientes con haplogrupo del Reino Unido, ninguno de estos dos factores fue un factor de riesgo significativo para RDP. Sin embargo, un estudio más reciente y más amplio sobre los mismos haplotipos no pudo mostrar tal asociación.

Hay menos acuerdo entre los estudios sobre la importancia de otros factores en el resultado del tratamiento anti-VEGF: tipo de diabetes, factores de coagulación, insuficiencia renal, inactividad física, biomarcadores inflamatorios, uso de IECA, microalbuminuria y tabaquismo.

Debido a la heterogeneidad en la respuesta a los antiangiogénicos y los esteroides en el tratamiento del EMD, futuros estudios sobre la correlación con los múltiples factores de riesgo asociados, tanto modificables (presión arterial, lípidos, HbA1c) como no modificables (daño cardiovascular, función renal, genética), proporcionarán información adicional para promover mejores resultados.

En la figura 1 se presenta un resumen de la información expuesta.

\section{Bibliografía}

1. Chew E, Ambrosius W, Davis M, Danis R, Gangaputra S, Greven C et al. Effects of medical therapies on retinopathy progression in type 2 diabetes. N Engl J Med. 2010;363:233-44. 
2. Diabetes Control and Complications Trial Research Group. The relationship of glycemic exposure ( $\mathrm{HbA} 1 \mathrm{c})$ to the risk of development and progression of retinopathy in the diabetes control and complications trial. Diabetes. 1995:44:968-83.

3. Stratton I, Adler A, Neil H, Matthews D, Manley S, Cull C, et al. Association of glycaemia with macrovascular and microvascular complications of type 2 diabetes (UKPDS 35): prospective observational study. BMJ. 2000;321:405-12.

4. Keech A, Mitchell P, Summanen P, O'Day J, Davis T, Maffitt S, et al. Effect of fenofibrate on the need for laser treatment for diabetic retinopathy (FIELD study): a randomised controlled trial. Lancet. 2007;370:1687-97.

5. Gerstein $\mathrm{H}$, Ambrosius W, Danis R, Beigi F, Cushman W, Calles J, et al. Diabetic retinopathy, its progression and incident cardiovascular events in the ACCORD trial. Diabetes Care. 2013;36:1266-71.

6. Motta A, Pajewski N, Fonseca V, Beigi F, Chew E, Ambrosius W, et al. The degree of retinopathy is equally predictive for renal and macrovascular outcomes in the ACCORD trial. J Diabetes Complications. 2014;28:874-9.

7. Klein R, Lee K, Knudtson M, Gangnon R, Klein B. Changes in visual impairment prevalence by period of diagnosis of diabetes: the Wisconsin Epidemiologic Study of Diabetic Retinopathy. Ophthalmology 2009; 116:1937-42.

8. Laiteerapong N, Ham S, Gao Y, Moffett H, Liu J, Huang E, et al. The legacy effect in type 2 diabetes: impact of early glycemic control on future complications (the Diabetes and Aging Study). Diabetes Care. 2019;42:416-26
9. Little R, Rohlfing C, Sacks D. Status of hemoglobin A1c measurement and goals for improvement: from chaos to order for improving diabetes care. Clin Chem. 2011;57:205-14.

10. Systolic Blood Pressure Intervention Trial (SPRINT). A randomized trial of intensive vs. standard blood pressure control. $\mathrm{N}$ Engl J Med. 2015;373:2103-16

11. Mauer M, Zinman B, Gardiner R, Suissa S, Sinaiko A, Strand T, et al. Renal and retinal effects of enalapril and losartan in type 1 diabetes. N Engl J Med. 2009;361:40-51

12. Pareja A, Serrano M, Marrero M, Abraldes V, Reyes M, Cabrera F, et al. Guías de práctica clínica de la SERV: manejo de las complicaciones oculares de la diabetes, retinopatía diabética y edema macular. Rev Soc Esp Oftalmol. 2009;84:429-50.

13. Diabetic Retinopathy Clinical Research Network. An observational study in individuals with diabetic retinopathy. Protocol T. Ophthalmic Res. 2019;62:225-30.

14. Bressler S, Odia I, Maguire M, Dhoot D, Glassman, Jampol L, et al. Factors associated with visual acuity and central subfield thickness changes when treating diabetic macular edema with anti-vascular endothelial growth factor therapy: an exploratory analysis of the Protocol $T$ randomized clinical trial. JAMA Ophthalmol. 2019;137:382-9. 


\title{
Anti-VEGF como tratamiento de primera línea y esteroides como tratamiento de segunda línea. Esteroides como tratamiento de primera línea y anti-VEGF como tratamiento de segunda línea
}

\author{
Jans Fromow-Guerra* \\ Asociación para Evitar la Ceguera en México (APEC), Ciudad de México, México
}

El edema macular diabético (EMD) es la principal causa de discapacidad visual en las personas en edad productiva. Este capítulo resume las consideraciones basadas en la evidencia de las razones para abordar esta enfermedad con medicamentos dirigidos contra el factor de crecimiento endotelial vascular (anti-VEGF [vascular endothelial growth factor]) y dejar la opción de tratamiento con esteroides para una segunda línea.

\section{Fármacos anti-VEGF}

Los anti-VEGF se han convertido en el tratamiento de referencia del EMD, reemplazando a la fotocoagulación con láser macular. Best, et al. ${ }^{1}$ demostraron la eficacia real del ranibizumab en el EMD a los 12 meses y la necesidad de una gran cantidad de inyecciones para lograr mejores resultados visuales. También observaron una tendencia a un menor cumplimiento por parte de los pacientes diabéticos en comparación con aquellos con degeneración macular relacionada con la edad neovascular: solo el $16.8 \%$ de estos últimos se perdieron durante el seguimiento al año, frente al $25-45 \%$ de los pacientes diabéticos.

Muchos ojos responden bien a los agentes antiVEGF y más de $80 \%$ de los pacientes tendrán algún tipo de respuesta, pero la mitad de estos no tendrán una resolución completa. De estos pacientes que no logran un control favorable del edema se dice que presentan EMD refractario. Cambiar de un fármaco anti-VEGF a otro es un primer paso viable para el manejo del EMD resistente.

Demircan, et al. ${ }^{2}$ compararon un grupo de pacientes que cambiaron a aflibercept después de mostrar una respuesta deficiente al tratamiento previo con ranibizumab frente a un grupo de pacientes que continuaron con inyecciones de ranibizumab a pesar de tener una pobre respuesta. Los resultados demostraron que el cambio de ranibizumab intravítreo a aflibercept en el EMD persistente solo proporcionó una mejoría morfológica. La discrepancia entre los resultados morfológicos y funcionales puede explicarse por el daño funcional irreversible causado por el EMD de larga evolución.

Sin embargo, a pesar de no lograr una respuesta de resolución en todos los pacientes y requerir múltiples inyecciones, los anti-VEGF tienen un buen perfil de seguridad y una eficacia satisfactoria en gran número de pacientes. Estas seguridad y eficacia están basadas en una gran experiencia en estudios clínicos con los tres principales medicamentos de este grupo: bevacizumab, ranibizumab y aflibercept. El primer estudio diseñado para estudiar el efecto de los anti-VEGF en el EMD fue el Protocolo T, cuyo propósito fue comparar la eficacia y la seguridad de aflibercept intravítreo, bevacizumab y ranibizumab para el tratamiento del EMD con involucro del centro de la mácula. El estudio incluyó 660 pacientes con una edad promedio de $61 \pm$ 
10 años, de los cuales el 90\% tenían diabetes mellitus tipo $2^{3,4}$. De acuerdo con los resultados a 1 año, el cambio promedio de letras de agudeza visual mejor corregida (AVMC) mejoró en 13.3 letras con aflibercept, 9.7 letras con bevacizumab y 11.2 letras con ranibizumab. Sin embargo, los mejores resultados generales para aflibercept en la población general no se consideraron relevantes clínicamente ni estadísticamente, debido a la interacción de la puntuación inicial de la AVMC y los resultados del tratamiento. Cuando la AVMC fue de 69 letras o más (equivalente de Snellen 20/40 o mejor), el cambio promedio de letras de AVMC mejoró en $8.0 \pm 7.6$ para aflibercept, $7.5 \pm 7.4$ para bevacizumab y $8.3 \pm 6.8$ para ranibizumab, sin ser estadísticamente significativa la diferencia entre los grupos de tratamiento. Para los ojos con una AVMC inicial de menos de 69 letras (equivalente de Snellen 20/50 o peor), la mejoría promedio fue de $18.9 \pm 11.5$ para aflibercept, $11.8 \pm 12.0$ para bevacizumab y $14.2 \pm 10.6$ para ranibizumab. Estos resultados tuvieron una clara diferencia significativa a favor del aflibercept, en comparación con el bevacizumab y el ranibizumab $(p=0.0001$ para aflibercept vs. bevacizumab, $p=0.0003$ para aflibercept vs. ranibizumab y $p=0.21$ para ranibizumab vs. bevacizumab). Por tanto, podemos concluir que el uso inicial de anti-VEGF está sustentado como primera línea de tratamiento.

\section{Esteroides}

Existe amplia evidencia que destaca el papel de la inflamación en el desarrollo de EMD. Los procesos patológicos incluyen leucostasis, que es la acumulación de leucocitos en la superficie de los capilares retinianos y se cree que es un mediador clave y un evento temprano en la disfunción de la barrera hematorretiniana. Dicha leucostasis conduce a la regulación positiva de la molécula de adhesión intracelular (ICAM)1, que media la atracción de monocitos y neutrófilos hacia el endotelio vascular. Se ha establecido que la ICAM-1 mejora aún más la leucostasis retiniana, la permeabilidad vascular y la degradación de la barrera hematorretiniana en los pacientes con diabetes. Los fármacos esteroides más estudiados en el EMD son el acetónido de triamcinolona, la dexametasona y el acetónido de fluocinolona. Aun con importantes diferencias en su farmacocinética y farmacodinámica, el implante de liberación prolongada de dexametasona y el acetónido de fluocinolona son los agentes que han demostrado la mejor relación eficacia-seguridad.
El estudio clínico PLACID, aleatorizado, controlado y multicéntrico, fue el primero que evaluó la dexametasona para el EMD. Un total de 253 pacientes con EMD con involucro central, con un puntaje de AVMC de 34-70 letras y grosor central de la retina $(C R T) \geq 275$ $\mu \mathrm{m}$, fueron asignados al azar para recibir el implante de liberación prolongada de dexametasona de $0.7 \mathrm{mg}$ y terapia con láser al mes 1 o monoterapia con láser con una inyección de implante simulado. Los pacientes podían recibir hasta tres tratamientos adicionales con láser y una inyección adicional de implante de liberación prolongada de dexametasona. Si bien a 1 año no hubo diferencias entre los grupos, el porcentaje de pacientes que obtuvo al menos 10 letras de mejoría fue significativamente mayor en el grupo de láser e implante de liberación prolongada de dexametasona, en comparación con el grupo de monoterapia con láser en el mes $1(31.7 \%$ frente a $11 \% ; p<0.001)$ y en el mes 9 (31.7\% frente a $17.3 \% ; p=0.007)$. El aumento de la presión intraocular se produjo con mayor frecuencia en el grupo de láser e implante de liberación prolongada de dexametasona que en el grupo de monoterapia con láser, con un $15.2 \%$ de pacientes con un aumento $\geq$ $10 \mathrm{mmHg}$ desde el inicio, un $16.8 \%$ con $\geq 25 \mathrm{mmHg}$ y un $4 \%$ con $\geq 35 \mathrm{mmHg}$. En el $15.9 \%$ de los pacientes en el grupo de láser e implante de liberación prolongada de dexametasona y el $1.6 \%$ de los pacientes del grupo de monoterapia con láser se inició tratamiento con medicamentos para reducir la presión intraocular. Cabe mencionar que ningún ojo requirió cirugía para controlar la presión intraocular. Los eventos adversos relacionados con cataratas fueron más comunes entre los pacientes fáquicos en el grupo de láser e implante de liberación prolongada de dexametasona (22.2\%) que en el grupo de monoterapia con láser (9.5\%). La cirugía de cataratas se realizó en cuatro ojos en el grupo de láser e implante de liberación prolongada de dexametasona y en cinco ojos en el grupo de monoterapia con láser ${ }^{5}$.

De acuerdo con los argumentos comentados, se puede afirmar que existen estrategias terapéuticas para el EMD que apoyan utilizar primero tratamiento con anti-VEGF por su alto porcentaje de respuesta $(80 \%)$, a pesar de que se requieren múltiples tratamientos para el control final, y dejar como segunda línea el uso de esteroides, que pudiera ofrecer la ventaja de una recuperación más rápida, pero con efectos colaterales como hipertensión ocular y catarata reportados con mayor frecuencia. 


\section{Bibliografía}

1. Best A, Fajnkuchen F, Nghiem S, Grenet T, Quentel G, Delahaye C, et al. Treatment efficacy and compliance in patients with diabetic macular edema treated with ranibizumab in a real-life setting. J Ophthalmol. 2018:2018:4610129

2. Demircan A, Alkin Z, Yesilkaya C, Demir G, Kemer B. Comparison of intravitreal aflibercept and ranibizumab following initial treatment with ranibizumab in persistent diabetic macular edema. J Ophthalmol. 2018;2018:4171628.
3. Aiello L, Beck R, Bressler N, Browning D, Chalam K, David M, et al. Rationale for the diabetic retinopathy clinical research network treatment protocol for center-involved diabetic macular edema. Ophthalmology. 2011;118:e5-14.

4. Wells J, Glassman A, Ayala A, Jampol L, Aiello L, Antoszyk A, et al. Aflibercept, bevacizumab or ranibizumab for diabetic macular edema. N Engl J Med. 2015;372:1193-203.

5. Callanan D, Gupta S, Boyer D, Siulla T, Singer M, Kuppermann B, et al. Dexamethasone intravitreal implant in combination with laser photocoagulation for the treatment of diffuse diabetic macular edema. Ophthalmology. 2013;120:1843-51. 


\title{
Rutas de decisión terapéutica (diagramas de flujo) en el edema macular diabético: sugerencias de conductas a seguir
}

\author{
Juan M. Jiménez-Sierra* \\ Asociación para Evitar la Ceguera en México (APEC), Ciudad de México, México
}

\section{¿Cómo seguir las rutas de decisiones terapéuticas?}

Estos diagramas están diseñados para ayudarnos a tomar de forma sencilla la mejor decisión terapéutica en casos de edema macular diabético (EMD). No pretenden ser estrictos ni dogmáticos; se sugieren como guías y se apoyan en la valoración clínica, la historia del paciente, los antecedentes, los estudios y la respuesta terapéutica a tratamientos previos. Además, han sido modificados y adaptados a nuestra población por varios retinólogos de países de habla hispana, y se reproducen con permiso de Allergan/AbbVie.

\section{¿Cómo entender los diagramas de flujo?}

Se basan en preguntas sencillas que debemos formularnos de manera individual ante cada caso particular. El criterio y la experiencia de cada especialista pueden modificar el camino a seguir, que siempre debe ser individualizado, recordando el principio de que «existen enfermos y no enfermedades».

\section{Primera pregunta}

Ante un paciente con diabetes tipo 2 y EMD, ¿cómo debemos proceder?

Se deben evaluar los siguientes aspectos antes de iniciar el tratamiento:

- Sistémicos: evolución de la diabetes mellitus tipo 2 (años), control de la hemoglobina glucosilada, complicaciones diabéticas y comorbilidad (hipertensión arterial, dislipidemia, síndrome metabólico, etc.).

- Oftalmológicos: agudeza visual, biomicroscopía, cristalino, vítreo, tratamiento oral (TO).

- Estudios:

- Tomografía de coherencia óptica (OCT) macular (tipo de edema, grosor macular central, interfase vitreorretiniana, integridad de las capas de la retina, biomarcadores por OCT para EMD).

- Angio-OCT: zona foveal avascular, áreas de cierre capilar, mapa de densidad vascular.

- Angiografía con fluoresceína: microaneurismas, escape de colorante, presencia de anormalidades microvasculares intrarretinianas, proliferaciones vasculares, áreas de cierre capilar periféricas.

Ya se detectó EMD. 


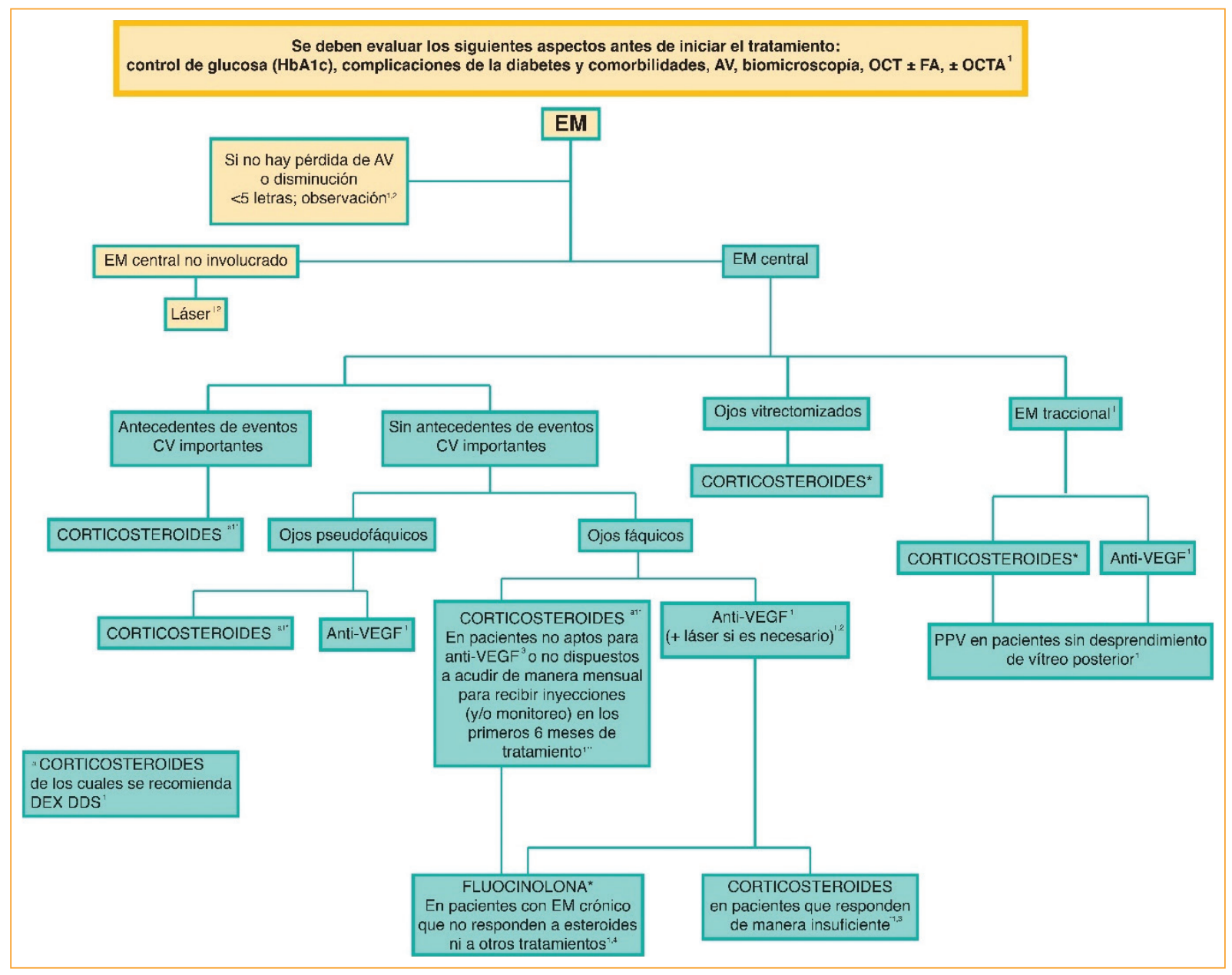

Figura 1. El edema macular (EM) diabético con buena agudeza visual (AV) y centro de la mácula no involucrado puede mantenerse en observación o aplicar láser focal. CV: cardiovasculares; DEX: dexametasona; DDS: drug delivery system; ETDRS: Early Treatment Diabetic Retinopathy Study, FA: fluorangiografía; HbA1c: hemoglobina glucosilada; OCT: tomografía de coherencia óptica; OCTA: angiografía por tomografía de coherencia óptica; PPV: vitrectomía de la pars plana; VEGF, factor de crecimiento vascular endotelial. (Adaptada de refs. 1-4.).

*No recomendado si presenta glaucoma avanzado preexistente o no controlado ${ }^{3,4}$.

'Tracción anterior-posterior.

‡Láser focal.

**Es necesario monitorear la presión intraocular.

\section{Segunda pregunta}

¿Sin pérdida de agudeza visual o con una disminución de esta $<5$ letras? Observación ${ }^{1,2}$.

Si el EMD no es central y se clasifica como focal, puede valorarse tratamiento láser directamente en los microaneurismas (EMD focal extra foveal = láser focal) u observación.

Seguiremos la ruta amarilla de la figura 1.

\section{Tercera pregunta}

¿El EMD involucra el centro de la mácula?

Tenemos cuatro opciones:

- Con antecedente de eventos cardiovasculares importantes: considerar no utilizar fármacos dirigidos contra el factor de crecimiento endotelial vascular (anti-VEGF, vascular endothelial growth factor) como primera línea, ya que podría aumentar los riesgos. 


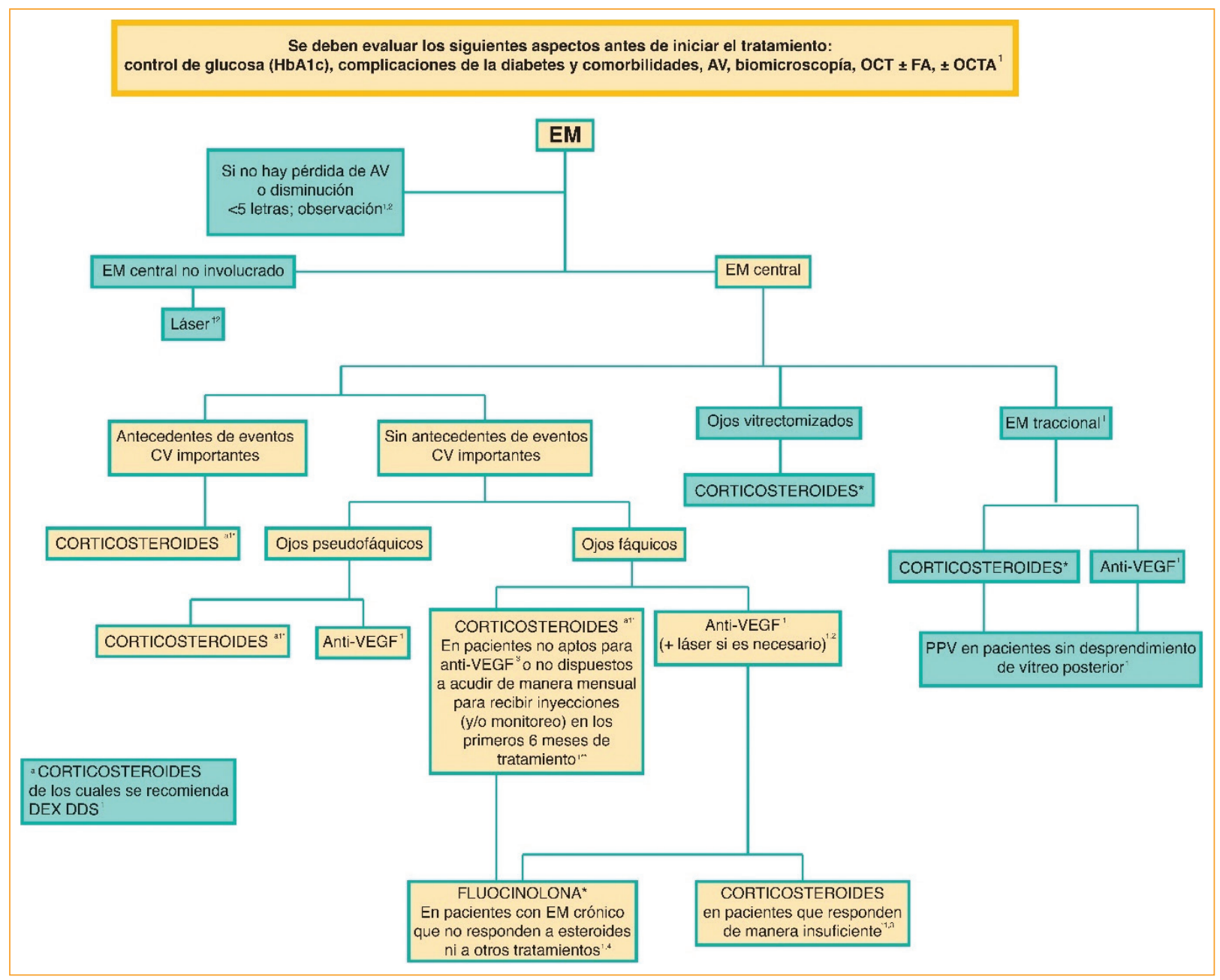

Figura 2. En caso de antecedente de eventos cardiovasculares (CV) importantes, considerar corticosteroides como primera línea. Sin antecedentes CV, hay dos opciones: 1) ojos pseudofáquicos, considerar esteroides 0 anti-VEGF; 2) ojos fáquicos, hay dos opciones, corticosteroides en pacientes con dificultad de inyección mensual y anti-VEGF más láser si es necesario. Si se decide anti-VEGF y la respuesta no es favorable después de tres inyecciones, cambiar a esteroide. AV: agudeza visual; DEX: dexametasona; DDS: drug delivery system; EM: edema macular; ETDRS: Early Treatment Diabetic Retinopathy Study, FA: fluorangiografía; HbA1c: hemoglobina glucosilada; OCT: tomografía de coherencia óptica; OCTA: angiografía por tomografía de coherencia óptica; PPV: vitrectomía de la pars plana; VEGF, factor de crecimiento vascular endotelial. (Adaptada de refs. 1-4.).

${ }^{*}$ No recomendado si presenta glaucoma avanzado preexistente o no controlado ${ }^{3,4}$.

'Tracción anterior-posterior.

‡Láser focal.

**Es necesario monitorear la presión intraocular.

Considerar como primera línea un esteroide intravítreo.

- Sin antecedente de eventos cardiovasculares:

- Ojos pseudofáquicos: en ojos operados de catarata con lente intraocular se pueden considerar de primera línea por igual los esteroides y los anti-VEGF.
- Ojos fáquicos: tenemos dos opciones:

- Corticosteroides: en pacientes considerados no aptos para anti-VEGF o que no están dispuestos a recibir inyecciones mensuales y monitoreo en los primeros 6 meses de tratamiento, y que pueden desarrollar o progresar catarata, si se consideran 


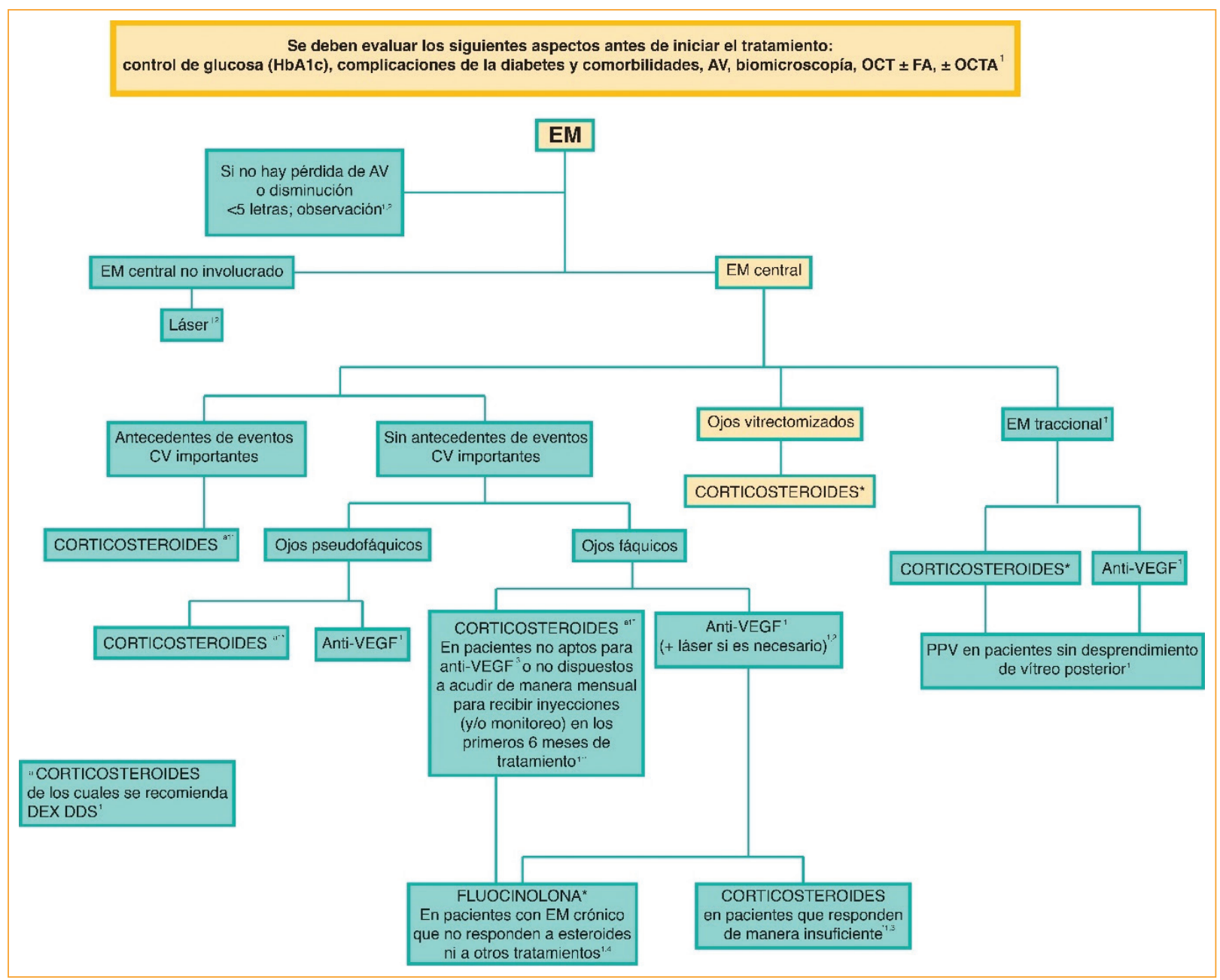

Figura 3. Ojos vitrectomizados; considerar esteroide como primera línea, ya que la vida media de los anti-VEGF se reduce de manera importante después de la vitrectomía. AV: agudeza visual; CV: cardiovasculares;

DEX: dexametasona; DDS: drug delivery system; EM: edema macular; ETDRS: Early Treatment Diabetic Retinopathy Study, FA: fluorangiografía; HbA1c: hemoglobina glucosilada; OCT: tomografía de coherencia óptica; OCTA: angiografía por tomografía de coherencia óptica; PPV: vitrectomía de la pars plana; VEGF, factor de crecimiento vascular endotelial. (Adaptada de refs. 1-4.).

${ }^{*}$ No recomendado si presenta glaucoma avanzado preexistente o no controlado ${ }^{3,4}$

'Tracción anterior-posterior

‡Láser focal.

${ }^{*}$ Es necesario monitorear la presión intraocular.

previamente para cirugía de catarata puede ser de primera línea el esteroide'.

- Anti-VEGF: considerar una inyección mensual hasta reducir el edema, y puede ser necesario láser ${ }^{1,2}$. Si la respuesta es no adecuada después de tres inyecciones mensuales (cerca del $40 \%$ de los pacientes), considerar el cambio a un esteroide ${ }^{1}$.

Seguiremos la ruta amarilla de la figura 2.

\section{Cuarta pregunta}

¿Qué hacer en ojos vitrectomizados?

Seguiremos la ruta amarilla de la figura 3 .

\section{Quinta pregunta}

¿Tiene el EMD componente traccional?

Seguiremos la ruta amarilla de la figura 4. 


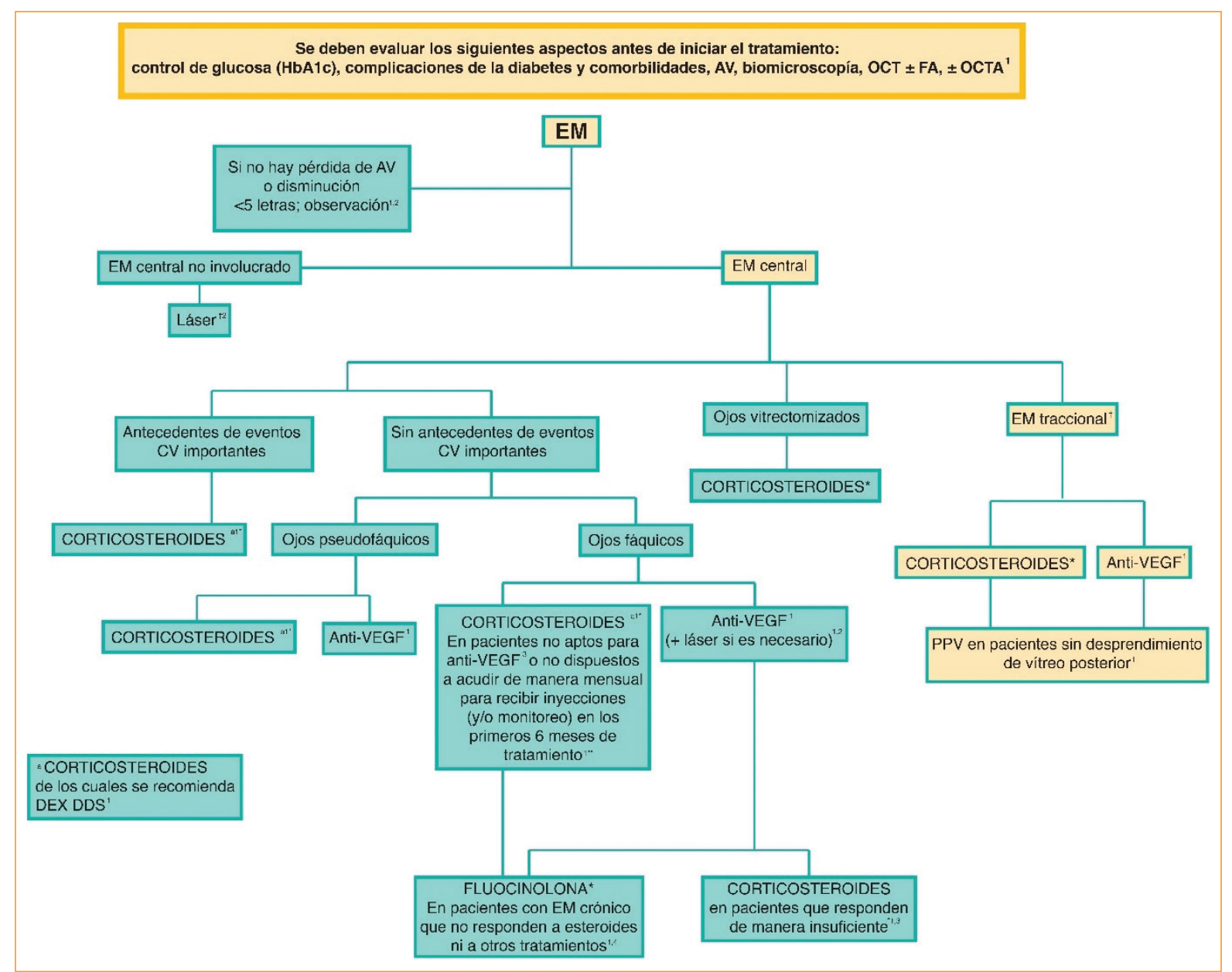

Figura 4. En el edema macular (EM) diabético traccional, si la tracción es importante, considerar vitrectomía pars plana. Cuando existe EM con membranas premaculares, se sugiere vitrectomía. Puede complementarse con esteroides intravítreos o anti-VEGF1. AV: agudeza visual; CV: cardiovasculares; DEX: dexametasona; DDS: drug delivery system; ETDRS: Early Treatment Diabetic Retinopathy Study, FA: fluorangiografía; HbA1c: hemoglobina glucosilada; OCT: tomografía de coherencia óptica; OCTA: angiografía por tomografía de coherencia óptica; PPV: vitrectomía de la pars plana; VEGF, factor de crecimiento vascular endotelial. (Adaptada de refs. 1-4.). *No recomendado si presenta glaucoma avanzado preexistente o no controlado ${ }^{3,4}$.

†Tracción anterior-posterior.

‡Láser focal.

**Es necesario monitorear la presión intraocular.

\section{Bibliografía}

1. Schmidt U, Garcia J, Bandello F, Berg K, Chakrabarthy U, Gerendas B et al. Guidelines for the management of diabetic macular edema by the European Society of Retina Specialists (EURETINA). Ophthalmologica. 2017;237:185-222.
2. ICO Guidelines for Diabetic Eye Care. International Council of Ophthalmology 2017. Disponible en: http://www.icoph.org/downloads/ICOGuideline_2017.

3. Ozurdex ${ }^{\circledR}$. Resumen de características del producto, 2015. Disponible en: https://www.aemps.gob.es.

4. Iluvien ${ }^{\circledR}$. Resumen de características del producto, 2016. Disponible en: https://www.cima.aemps.es. 